Supporting Information for

\title{
A Stereodivergent Methodology for the Synthesis of Complex Pyrrolidines
}

\author{
Stephen K. Jackson, Avedis Karadeolian, Alex B. Driega and Michael A. Kerr*
}

The University of Western Ontario

London, Ontario, Canada

N6A $5 B 7$ 


\section{Table of Contents}

General Experimental Remarks

General Procedure A- oxime ether synthesis .

General Procedure B- synthesis of 2,5-trans pyrrolo-isoxazolidine adducts ........................................ S3

General Procedure C- synthesis of 2,5-cis-pyrrolo-isoxazolidine adducts............................................ S3

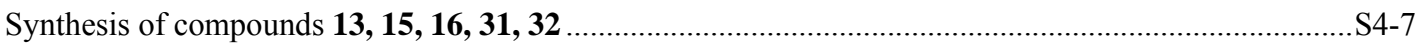

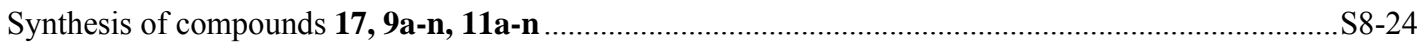

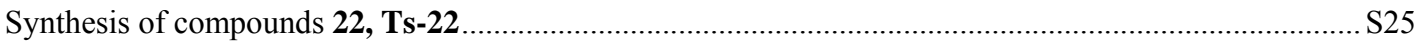

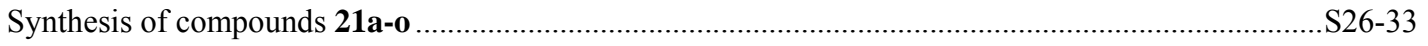

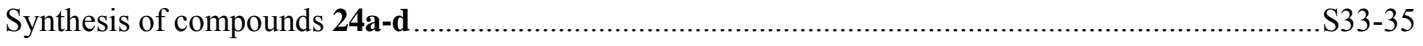

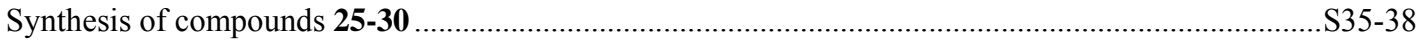

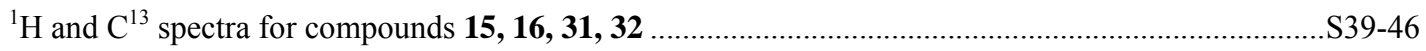

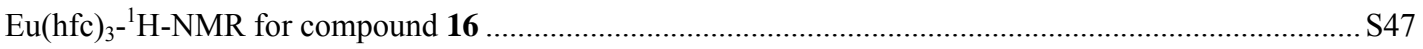

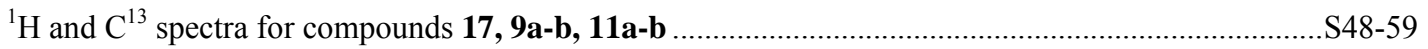

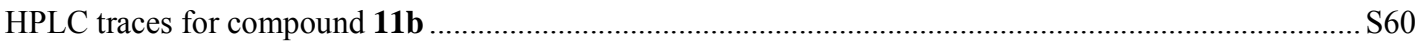

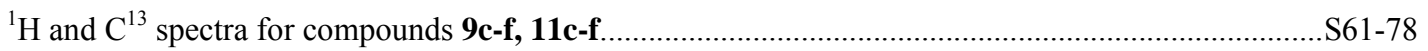

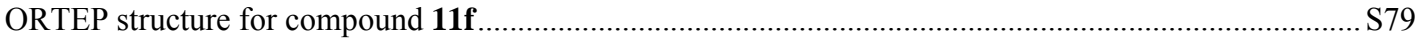

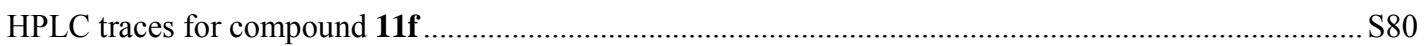

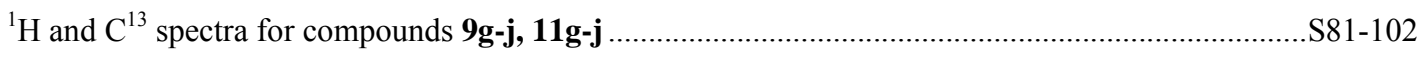

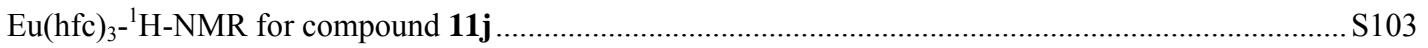

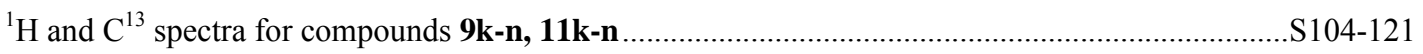

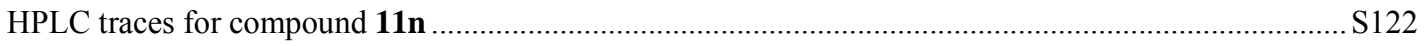

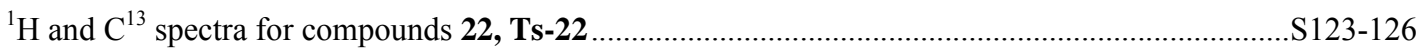

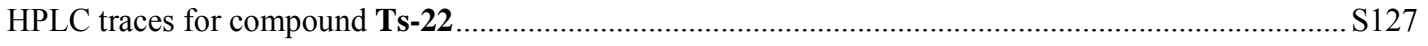

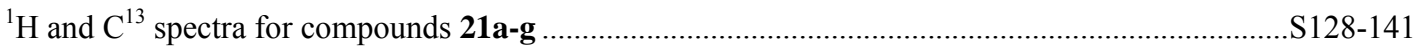

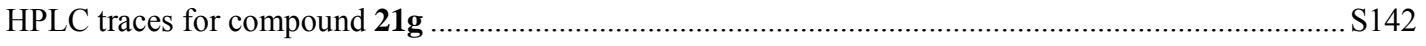

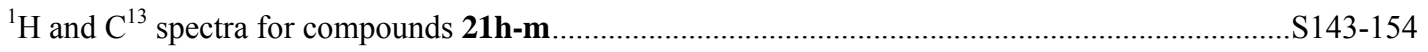

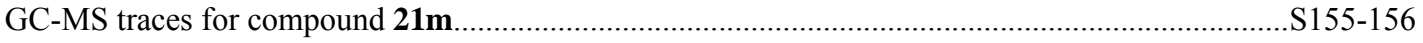

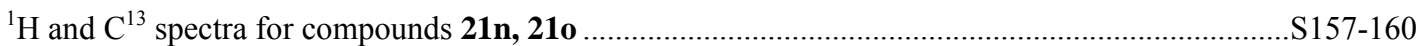

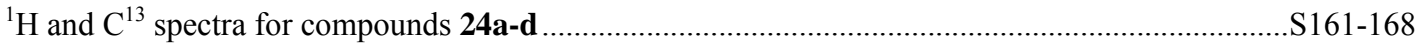

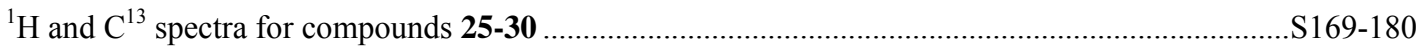




\section{General Remarks}

Melting points are uncorrected. Infrared spectra were obtained as thin films on $\mathrm{NaCl}$ plates. NMR experiments were performed on 400 or $600 \mathrm{MHz}$ instruments. Spectra recorded in $\mathrm{CDCl}_{3}$ were referenced to residual $\mathrm{CHCl}_{3}$ at $7.26 \mathrm{ppm}$ for ${ }^{1} \mathrm{H}$ or $77.0 \mathrm{ppm}$ for ${ }^{13} \mathrm{C}$. Spectra recorded in $\mathrm{d}_{6}$-DMSO were referenced to residual DMSO at 2.49 ppm for ${ }^{1} \mathrm{H}$ or 40.0 ppm for ${ }^{13} \mathrm{C}$. Coupling constants $(J)$ are in Hz. The multiplicities of the signals are described using the following abbreviations: $\mathrm{s}=$ singlet, $\mathrm{d}=$ doublet, $\mathrm{t}=$ triplet, $\mathrm{q}=$ quartet, $\mathrm{m}=$ multiplet, $\mathrm{dd}=$ doublet of doublets, $\mathrm{br}=$ broad. Highresolution mass spectra (HRMS) were obtained at $70 \mathrm{eV}$. THF, ether, DMF and methylene chloride were dried and deoxygenated by passing the nitrogen-purged solvents through activated alumina columns. All other commercial reagents and solvents were used as obtained without further purification. The progress of reactions was followed by thin layer chromatography (TLC) (silica gel $60 \mathrm{~F}_{254}$ ) and the developed plates stained using acidic anisaldehyde, phosphomolybdic acid, or basic potassium permanganate. Flash chromatography was performed using silica gel (230400 mesh). All reported compounds are $>95 \%$ pure as judged by ${ }^{1} \mathrm{H}$ NMR.

\section{General Procedure A- synthesis of oxime-ethers 9}

The aldehyde (1.1 eq.) and cyclopropyl-alkoxylamine 17 (1.0 eq.) were dissolved in a 3:1 mixture of $\mathrm{CH}_{2} \mathrm{Cl}_{2} / \mathrm{MeOH}$ $(0.13 \mathrm{M})$ and stirred over $\mathrm{MgSO}_{4}(3.5 \mathrm{eq})$. The reaction was monitored by ${ }^{1} \mathrm{H} \mathrm{NMR}$ and upon consumption of the cyclopropane, the reaction mixture was filtered, pre-absorbed onto silica and purified by flash column chromatography (elution EtOAc/hexanes). The geometrical assignment of the oxime either was made on basis of ${ }^{1} \mathrm{H}$ chemical shift of aldoxime proton. ${ }^{1}$

\section{General Procedure B- synthesis of 2,5-trans-pyrrolo-isoxazolidines 11}

Oxime-ether 9 (1.0 eq.) was dissolved in dry $\mathrm{CH}_{2} \mathrm{Cl}_{2}(0.1 \mathrm{M})$ and $\mathrm{Yb}(\mathrm{OTf})_{3} \bullet \mathrm{xH}_{2} \mathrm{O}(5 \mathrm{~mol} \%)$ was added. The mixture was stirred overnight at room temperature (some reactions were heated to $40{ }^{\circ} \mathrm{C}$ if not complete after 16 hours at room temperature) and monitored by TLC for completion. The reaction mixture was diluted with $\mathrm{CH}_{2} \mathrm{Cl}_{2}$, washed with $\mathrm{H}_{2} \mathrm{O}$ (1x), brine (1x), dried over $\mathrm{MgSO}_{4}$ and concentrated in vacuo. The crude product was pre-absorbed onto silica and purified by flash column chromatography (elution EtOAc/hexanes).

General Procedure C-s synthesis of 2,5-cis-pyrrolo-isoxazolidines 21

To a solution of cyclopropyl-alkoxylamine 17 (1.0 eq) in $\mathrm{CH}_{2} \mathrm{Cl}_{2}(0.2 \mathrm{M})$ was added $\mathrm{Yb}(\mathrm{OTf})_{3}(5 \mathrm{~mol} \%)$. After stirring for 30 minutes at room temperature, the aldehyde (1.1 eq.) was then added and the reaction mixture stirred for 18 hrs or

${ }^{1}$ Karabatsos, G. J.; Hsi, N. Tetrahedron, 1967, 23, 1079-1095. 
until complete by TLC or ${ }^{1} \mathrm{H}$ NMR analysis. The mixture was concentrated, preabsorbed onto silica gel and purified by flash column chromatography (EtOAC/hexanes elution).

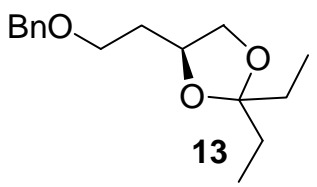

Compound 13 was prepared as follows according to a modified literature procedure. ${ }^{2 \mathrm{a}-\mathrm{c}}$ To a solution of $(S)$-butane triol (10.0 g, $94.2 \mathrm{mmol})$ in 3-pentanone $(460 \mathrm{ml})$ was added $p$-toluene sulfonic acid $(0.54 \mathrm{~g}, 2.8 \mathrm{mmol})$ and $4 \AA \mathrm{MS}(28.75 \mathrm{~g})$. The reaction mixture was stirred for $18 \mathrm{hrs}$ after which $\mathrm{NEt}_{3}(0.40 \mathrm{ml})$ was added. The solution was stirred for an additional $10 \mathrm{~min}$ and then concentrated in vacuo to $150 \mathrm{ml}$. The sieves were removed by filtration through Celite and rinsed with EtOAc. The solution was further concentrated to remove the pentanone and the crude material was diluted with EtOAc and passed through a short plug of $\mathrm{SiO}_{2}$, eluting with EtOAc. Concentration afforded $23.70 \mathrm{~g}(96 \%)$ of the pure ketal, whose spectral data was identical to that reported. ${ }^{2 \mathrm{a}, \mathrm{c}}$ To a suspension of $\mathrm{NaH}(6.26 \mathrm{~g}, 60 \%$ dispersion in oil, $156 \mathrm{mmol})$ in THF $(200 \mathrm{ml})$ was added the ketal $(23.7 \mathrm{~g}, 136 \mathrm{mmol})$ dropwise and the transfer quantitated with THF (40 $\mathrm{ml})$. After stirring for $10 \mathrm{~min}, \mathrm{BnBr}(17.8 \mathrm{ml}, 150.0 \mathrm{mmol})$ was added dropwise and the reaction was brought to reflux for $18 \mathrm{hrs}$. The reaction mixture was cooled to room temperature, diluted with $\mathrm{H}_{2} \mathrm{O}$ and concentrated to $1 / 4$ volume in vaccuo. The mixture was extracted with EtOAc (3x) and the combined organic fractions were washed with brine (1x), dried over $\mathrm{MgSO}_{4}$, and concentrated in vaccuo. The benzylated ketal 13 was purified by fractional vacuum distillation $\left(141-143{ }^{\circ} \mathrm{C}, 0.05 \mathrm{~mm} \mathrm{Hg}\right)$ to afford $31.7 \mathrm{~g}(93 \%)$ of the title compound as a yellow oil whose spectroscopic data was identical to that reported. ${ }^{2 \mathrm{~d}}$

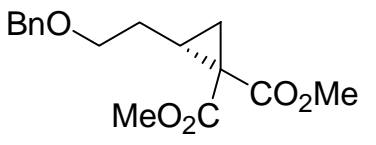

31

To a solution of compound $13(31.7 \mathrm{~g}, 120 \mathrm{mmol})$ in $\mathrm{MeOH}(405 \mathrm{ml})$ was added concentrated $\mathrm{HCl}(106 \mathrm{ml})$. The reaction mixture was heated to $50{ }^{\circ} \mathrm{C}$ and stirred for 2 hrs. The mixture was diluted with $\mathrm{H}_{2} \mathrm{O}$, cooled to $0{ }^{\circ} \mathrm{C}$ and

2 a) Arth, H. -L.; Sinerius, G.; Fessner, W. -D. Liebigs Ann. 1995, 2037-2042; b) Meyers, A. I.; Lawson, J. P. Tetrahedron Lett. 1982, 23, 4883-4886; c) Borjesson, L.; Csoregh, I; Welch, C. J. J. Org. Chem. 1995, 60, 2989-2999; d) Sasyou, M.; Naito, T. PCT Int. Appl. 2002, 35 pp., WO 20022088105. 
neutralized with the portionwise addition of $\mathrm{Na}_{2} \mathrm{CO}_{3}$. The solution was concentrated in vacuo to remove the $\mathrm{MeOH}$ and the aqueous mixture was extracted with EtOAc $(4 \mathrm{x})$. The combined organic fractions were washed with brine $(1 \mathrm{x})$, dried over $\mathrm{MgSO}_{4}$ and concentrated in vacuo. The crude product was purified by column chromatography $(100 \%$ EtOAc elution) to afford $21.9 \mathrm{~g}(100 \%)$ of the diol. The intermediate diol $(5.00 \mathrm{~g}, 27.44 \mathrm{mmol})$ was dissolved in $\mathrm{CCl}_{4}(25 \mathrm{ml})$ in a dry two-necked flask. One neck was stoppered with a septum, and a reflux condenser was place in the second neck. A bent glass tube filled with Drierite was placed on top of the reflux condenser and was connected via plastic tubing to a $\mathrm{KOH}$ trap. Thionyl chloride $(2.41 \mathrm{~mL}, 32.93 \mathrm{mmol})$ was added slowly via syringe to the solution and heated to $77{ }^{\circ} \mathrm{C}$ for $30 \mathrm{~min}$. The reaction was then cooled to $0{ }^{\circ} \mathrm{C}$ in an ice bath followed by the addition of $\mathrm{CH}_{3} \mathrm{CN}(25 \mathrm{ml}) . \mathrm{RuCl}_{3} \bullet \mathrm{H}_{2} \mathrm{O}$ ( $8 \mathrm{mg}, 0.027 \mathrm{mmol}), \mathrm{NaIO}_{4}(8.80 \mathrm{~g}, 41.16 \mathrm{mmol})$, and $\mathrm{H}_{2} \mathrm{O}(37.5 \mathrm{ml})$ were added sequentially to the solution. ${ }^{3}$ The reaction was stirred vigorously at room temperature and monitored by TLC. Upon consumption of the cyclic sulphite $\left(\mathrm{R}_{\mathrm{f}}\right.$ $=0.60,50 \% \mathrm{EtOAc} / \mathrm{hexanes}), \mathrm{Et}_{2} \mathrm{O}(200 \mathrm{ml})$ was added to the reaction mixture and the layers allowed to separate. The organic layer was washed with $\mathrm{H}_{2} \mathrm{O}(1 \mathrm{x})$, saturated $\mathrm{NaHCO}_{3}(1 \mathrm{x})$, brine $(1 \mathrm{x})$, dried over $\mathrm{MgSO}_{4}$ and concentrated. The crude air sensitive sulphate $14\left(\mathrm{R}_{\mathrm{f}}=0.71,50 \%\right.$ EtOAc/hexanes $)$ was immediately diluted with $15 \mathrm{~mL}$ of $\mathrm{DME}$. Another flask was charge with $\mathrm{NaH}(2.26,56.6 \mathrm{mmol}, 60 \%$ dispersion in oil), suspended in DME (90 ml) and cooled to $0{ }^{\circ} \mathrm{C}$. Dimethyl malonate $(2.94 \mathrm{~mL}, 25.75 \mathrm{mmol})$ was added to the suspension drop-wise and stirred until gas evolution ceased. The cyclic sulphate solution (6.65 g in $15 \mathrm{ml} \mathrm{DME})$ was then added and the solution was heated to $84{ }^{\circ} \mathrm{C}$ for 24 hrs. The reaction mixture was cooled to room temperature, diluted with $\mathrm{H}_{2} \mathrm{O}$ and extracted with EtOAc (3x). The combined organic fractions were washed with $1 \mathrm{M} \mathrm{NaOH}(2 \mathrm{x}), \mathrm{H}_{2} \mathrm{O}(1 \mathrm{x})$, brine $(1 \mathrm{x})$, dried over $\mathrm{MgSO}_{4}$ and concentrated in vacuo. Purification by flash column chromatography (30\% EtOAc/hexanes elution) afforded $4.80 \mathrm{~g}(60 \%$ from 13) of benzyloxy-cyclopropane 31 as a yellow oil. ${ }^{1} \mathrm{H}-\mathrm{NMR}\left(600 \mathrm{MHz}, \mathrm{CDCl}_{3}\right) ; \delta=7.35-7.32(\mathrm{~m}, 4 \mathrm{H}), 7.29-7.26(\mathrm{~m}, 1 \mathrm{H})$, $4.51(\mathrm{~s}, 2 \mathrm{H}), 3.72(\mathrm{~s}, 3 \mathrm{H}), 3.71(\mathrm{~s}, 3 \mathrm{H}), 3.54(\mathrm{t}, J=6.6 \mathrm{~Hz}, 2 \mathrm{H}), 2.04$ (dddd, $\left.J_{1-4}=7.8 \mathrm{~Hz}, 1 \mathrm{H}\right), 1.78$ (dddd, $J=13.8,6.6$, 6.6, $6.6 \mathrm{~Hz}, 1 \mathrm{H}), 1.53(\mathrm{dddd}, J=14.4,7.2,7.2,7.2 \mathrm{~Hz}, 1 \mathrm{H}), 1.45-1.41(\mathrm{~m}, 2 \mathrm{H}) ;{ }^{13} \mathrm{C}-\mathrm{NMR}\left(100 \mathrm{MHz}, \mathrm{CDCl}_{3}\right) ; \delta=170.6$, $168.6,138.3,128.3,127.6,127.5,72.9,69.0,52.5,52.4,33.6,29.0,25.7,20.9$; IR (thin film) $v_{\max }=3065,3031,2953$, $2859,1727,1497,1437,1363,1291,1213,1130 ;$ HRMS calc. for $\mathrm{C}_{16} \mathrm{H}_{20} \mathrm{O}_{5}=292.1311$, exp. $=292.1313 ;(S)-31,[\alpha]_{\mathrm{D}}^{22}$ $=-18.3^{\circ}\left(\mathrm{c} 8.0, \mathrm{CH}_{2} \mathrm{Cl}_{2}\right)$.

\footnotetext{
${ }^{3}$ For the oxidation of cyclic sulphites, see: Gao, Y.; Sharpless, K. B. J. Am. Chem. Soc. 1988, 110, 7538-7539.
} 


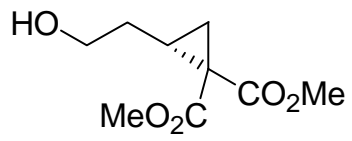

15

To a solution of the benzyloxy-cyclopropane 31 (9.32 g, $31.88 \mathrm{mmol})$ in $\mathrm{MeOH}(37 \mathrm{ml})$ was added Pd/C (0.932 g of 10 wt $\% \mathrm{Pd} / \mathrm{C})$. The flask was evacuated and purged with $\mathrm{H}_{2}$ through four cycles and then stirred at room temperature under a balloon ( $1 \mathrm{~atm})$ of $\mathrm{H}_{2}$ for $18 \mathrm{hrs}$. The solution was filtered through Celite, rinsed with $\mathrm{MeOH}$ and concentrated to afford $6.44 \mathrm{~g}(100 \%)$ of the pure title alcohol $(S)-15$ as a colourless oil.

$(+/-)-15$ is readily prepared as follows: To a chilled $\left(0^{\circ} \mathrm{C}\right)$ solution of $\mathrm{BH}_{3} \cdot \mathrm{SMe}_{2}(37.7 \mathrm{ml}, 0.40 \mathrm{~mol})$ and $\mathrm{BHT}(0.01 \mathrm{~g}$, $0.04 \mathrm{mmol})$ in THF (1.0 1) was added over $10 \mathrm{~min}$ a solution of (+/-)-2-vinylcyclopropane-1,1-dicarboxylic acid dimethyl $\operatorname{ester}^{4}(36.83 \mathrm{~g}, 0.20 \mathrm{~mol})$ in $\mathrm{THF}(200 \mathrm{ml})$. The reaction mixture was stirred at $0{ }^{\circ} \mathrm{C}$ for $2.5 \mathrm{hrs}$ and then carefully quenched with the dropwise addition of 1:1 MeOH/THF $(100 \mathrm{ml})$ over $1 \mathrm{hr}$. A precooled $\left(0{ }^{\circ} \mathrm{C}\right)$ aqueous phosphate buffer ( $\mathrm{pH} 7)$ solution (preparation of buffer: $\mathrm{KH}_{2} \mathrm{PO}_{4}(50.0 \mathrm{~g}, 0.37 \mathrm{~mol})$ and $\mathrm{NaOH}(8.4 \mathrm{~g}, 0.21 \mathrm{~mol}) \mathrm{made}$ up to $400 \mathrm{ml}$ with $\mathrm{H}_{2} \mathrm{O}$ ) was then carefully added to the reaction mixture followed by the addition of $30 \% \mathrm{H}_{2} \mathrm{O}_{2}$ (aq.) $(140 \mathrm{ml}, 1.2 \mathrm{~mol})$. The reaction mixture was slowly warmed to room temperature and stirred overnight. The solution was then concentrated in vacuo to $300 \mathrm{ml}$ and them partitioned between EtOAc and $\mathrm{H}_{2} \mathrm{O}$ (gentle shaking). The aqueous solution was extracted with EtOAc $(2 \mathrm{x})$ and the combined organic fractions were washed with saturated $\mathrm{Na}_{2} \mathrm{SO}_{3}(1 \mathrm{x})$, brine (1x), dried over $\mathrm{MgSO}_{4}$ and concentrated in vacuo. Purification by flash chromatography (elution $50 \%$, then $75 \%$ EtOAc/hexanes) through a short column afforded $25.4 \mathrm{~g}(63 \%)$ of (+/-)-15. ( ${ }^{1} \mathrm{H}-\mathrm{NMR}\left(400 \mathrm{MHz}, \mathrm{CDCl}_{3}\right) ; \delta=3.73(\mathrm{~s}$, 3H), 3.71-3.67 (m, 5H), 2.12 (br s, 1H), 1.97 (dddd, $\left.J_{1-4}=8.0 \mathrm{~Hz}, 1 \mathrm{H}\right), 1.66$ (dddd, J=14.0, 7.2, 7.2, $\left.7.2 \mathrm{~Hz}, 1 \mathrm{H}\right), 1.51$ $\left(\right.$ dddd, $\left.\left.J_{1-4}=7.2 \mathrm{hz}, 1 \mathrm{H}\right), 1.43(\mathrm{dd}, J=9.2,4.8 \mathrm{~Hz}, 1 \mathrm{H}), 1.38(\mathrm{dd}, J=8.0,4.8 \mathrm{~Hz}, 1 \mathrm{H}) ;{ }^{13} \mathrm{C}-\mathrm{NMR}\left(100 \mathrm{MHz}^{\mathrm{CDCl}}\right)_{3}\right) \delta=$ $170.6,168.7,61.7,52.6,52.5,33.5,31.6,25.5,20.6$; IR (thin film) $v_{\max }=3520,3007,2955,2882,1735,1437,1394$, 1290, 1213, 1126, 1076, 1046; HRMS calc. for $\mathrm{C}_{9} \mathrm{H}_{15} \mathrm{O}_{5}{ }^{++}=203.0920$, exp. $=203.0927$.

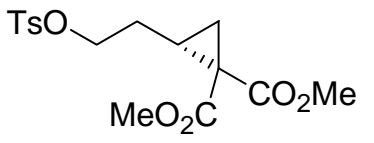

32

To a chilled $\left(0^{\circ} \mathrm{C}\right)$ solution of alcohol $15(6.12 \mathrm{~g}, 30.3 \mathrm{mmol})$ in $\mathrm{CH}_{2} \mathrm{Cl}_{2}(150 \mathrm{ml})$ was added DABCO (3.73 g, 33.29 mmol) followed by the portionwise addition of $\mathrm{TsCl}(6.34 \mathrm{~g}, 33.29 \mathrm{mmol})$. The reaction was stirred at $0{ }^{\circ} \mathrm{C}$ until $\mathrm{TLC}$

\footnotetext{
${ }^{4}$ For a one-step preparation of the vinyl cyclopropane diester, see: Miura, K.; Fujisawa, N.; Hosomi, A. J. Org. Chem. 2004, 69, 2427-2430.
} 
analysis showed complete consumption of the alcohol. The reaction mixture was diluted with $\mathrm{Et}_{2} \mathrm{O}(60 \mathrm{ml})$ and the resulting precipitate was removed by filtration through Celite. The filtrate was washed with $5 \% \mathrm{HCl}$ (aq.) (2x), saturated $\mathrm{NaHCO}_{3}$ (aq.) (1x), brine (1x) and dried over $\mathrm{MgSO}_{4}$. Concentration in vacuo afforded $21.54 \mathrm{~g}(93 \%)$ of tosylate 32 as a light yellow oil requiring no further purification. ${ }^{1} \mathrm{H}-\mathrm{NMR}\left(600 \mathrm{MHz}, \mathrm{CDCl}_{3}\right) ; \delta=7.76(\mathrm{~d}, J=8.4 \mathrm{~Hz}$, 2H), $7.33(\mathrm{~d}, J=8.4,2 \mathrm{H}), 4.05(\mathrm{t}, J=6.6 \mathrm{~Hz}, 2 \mathrm{H}), 3.69(\mathrm{~s}, 3 \mathrm{H}), 3.68(\mathrm{~s}, 3 \mathrm{H}), 2.49(\mathrm{~s}, 3 \mathrm{H}), 1.87\left(\mathrm{dddd}, J_{1-4}=7.8 \mathrm{~Hz}, 1 \mathrm{H}\right)$, 1.76 (dddd, $J=13.2,6.6,6.6,6.6 \mathrm{~Hz}, 1 \mathrm{H}), 1.61(\mathrm{dddd}, J=13.2,6.6,6.6,6.6 \mathrm{~Hz}, 1 \mathrm{H}), 1.36-1.31(\mathrm{~m}, 2 \mathrm{H}) ;{ }^{13} \mathrm{C}-\mathrm{NMR}(100$ $\left.\mathrm{MHz}, \mathrm{CDCl}_{3}\right) ; \delta=170.0,168.2,144.8,132.7,129.8,127.8,69.0,52.6$ (2C), 33.3, 28.1, 24.2, 21.5, 20.3; IR (thin film) $v_{\max }=3030,3003,2983,2956,2850,1734,1599,1456,1362,1294,1189 ;$ HRMS calc. for $\mathrm{C}_{16} \mathrm{H}_{20} \mathrm{O}_{7} \mathrm{~S}=356.0930$, exp. $=356.0923$.

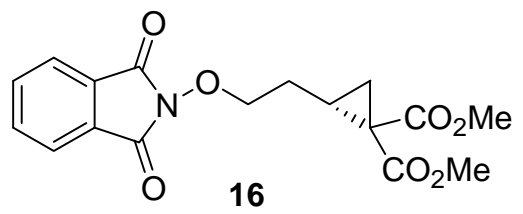

To a solution of $N$-hydroxyphthalimide $(5.18 \mathrm{~g}, 31.74 \mathrm{mmol})$ in DMF (50 ml) was added dropwise over $5 \mathrm{minutes}$ DBU (4.66 ml, $31.74 \mathrm{mmol})$. The solution was stirred for 5 minutes after which a solution of tosylate $32(10.1 \mathrm{~g}, 28.34 \mathrm{mmol})$ in DMF (50 ml) was added via cannula. The transfer was quantitated with additional DMF (25 ml) and the resulting mixture was stirred at room temperature for $48 \mathrm{hrs}$. The mixture was partitioned between EtOAc and $\mathrm{H}_{2} \mathrm{O}$ and the aqueous fraction was extracted with EtOAc (3x). The combined organic fractions were washed with $\mathrm{H}_{2} \mathrm{O}(4 \mathrm{x})$, brine (1x), dried over $\mathrm{MgSO}_{4}$ and concentrated in vacuo. The crude yellow solid was recrystallized from MeOH to afford 12.5 $\mathrm{g}$ of compound $\mathbf{1 6}$ as a white solid. The filtrate was concentrated and the remaining solid was purified by flash column chromatography (gradient elution, 1-8 \% EtOAc/hexanes) to yield an addition $1.9 \mathrm{~g}$ of compound 16 (14.4 g total yield, 74 \%). ${ }^{1} \mathrm{H}-\mathrm{NMR}\left(600 \mathrm{MHz}, \mathrm{CDCl}_{3}\right) ; \delta=7.84-7.82(\mathrm{~m}, 2 \mathrm{H}), 7.54-7.40(\mathrm{~m}, 2 \mathrm{H}), 4.28(\mathrm{t}, J=6.6 \mathrm{~Hz}, 2 \mathrm{H}), 3.76(\mathrm{~s}, 3 \mathrm{H})$, $3.73(\mathrm{~s}, 3 \mathrm{H}), 2.18\left(\mathrm{dddd}, J_{1-4}=7.2 \mathrm{~Hz}, 1 \mathrm{H}\right), 1.91(\mathrm{dddd}, J=13.8,6.6,6.6,6.6 \mathrm{~Hz}, 1 \mathrm{H}), 1.80$ (dddd, $J=13.8,6.6,6.6,6.6$ $\mathrm{Hz}, 1 \mathrm{H}), 1.53(\mathrm{dd}, J=9.0,4.8 \mathrm{~Hz}, 1 \mathrm{H}), 1.47(\mathrm{dd}, J=7.8,4.8 \mathrm{~Hz}, 1 \mathrm{H}) ;{ }^{13} \mathrm{C}-\mathrm{NMR}\left(100 \mathrm{MHz}, \mathrm{CDCl}_{3}\right) ; \delta=170.3,168.5$, $163.5,134.5,128.9,123.5,77.2,52.7,52.6,33.7,27.6,24.7,20.8 ;$ IR (thin film) $v_{\max }=3008,2956,2896,2850,1791$, 1733, 1468, 1438, 13345, 1216, 1188, 1131; HRMS calc. for $\mathrm{C}_{17} \mathrm{H}_{18} \mathrm{NO}_{7}{ }^{++}=348.1083$, exp. $=348.1094$; White solid. $(+/-$ )-16, $\mathrm{mp}=101-102{ }^{\circ} \mathrm{C} ;(\mathrm{S})-\mathbf{1 6} \mathrm{mp}=96-97^{\circ} \mathrm{C} ;[\alpha]_{\mathrm{D}}^{22}=-18.5^{\circ}\left(\mathrm{c} 5.9, \mathrm{CH}_{2} \mathrm{Cl}_{2}\right) ; \%$ ee $=>99 \%$, based on $(+)-\mathrm{Eu}(\mathrm{hcf})_{3}$ ${ }^{1} \mathrm{H}-\mathrm{NMR}$ analysis of the well separated methyl ester signals (vide infra for spectra). 


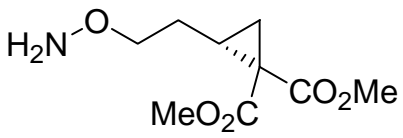

17

To a solution of phthalimide $\mathbf{1 6}(1.6 \mathrm{~g}, 4.6 \mathrm{mmol})$ in $\mathrm{MeOH} / \mathrm{CH}_{2} \mathrm{Cl}_{2}(1: 1,28 \mathrm{ml})$ was added hydrazine hydrate $(0.24 \mathrm{ml}$, $4.8 \mathrm{mmol}$ ). The reaction mixture was stirred at room temperature for 5 hrs after which $2 \% \mathrm{H}_{2} \mathrm{SO}_{4}$ (aq.) (80 ml) was added along with $\mathrm{CH}_{2} \mathrm{Cl}_{2}(20 \mathrm{ml})$. The mixture was filtered through Celite to remove the precipitate and the filter cake was washed with $2 \% \mathrm{H}_{2} \mathrm{SO}_{4}$ (aq.). The filtrate was extracted with $\mathrm{CH}_{2} \mathrm{Cl}_{2}(2 \mathrm{x})$ and the remaining aqueous fraction was neutralized with the addition of solid $\mathrm{Na}_{2} \mathrm{CO}_{3}$. The neutralized aqueous solution was then extracted with $\mathrm{CH}_{2} \mathrm{Cl}_{2}(3 \mathrm{x})$ and the combined organic fractions were dried over $\mathrm{MgSO}_{4}$ and concentrated to afford $0.92 \mathrm{~g}(92 \%)$ of the title cyclopropyl-alkoxylamine 17 as a yellow oil requiring no further purification. ${ }^{1} \mathrm{H}-\mathrm{NMR}\left(600 \mathrm{MHz}, \mathrm{CDCl}_{3}\right) ; \delta=5.37(\mathrm{br}$ s, $2 \mathrm{H}), 3.74(\mathrm{~s}, 3 \mathrm{H}), 3.73-3.70(\mathrm{~m}, 5 \mathrm{H}), 1.96\left(\mathrm{dddd}, J_{1-4}=7.8 \mathrm{~Hz}, 1 \mathrm{H}\right), 1.73(\mathrm{dddd}, J=13.2,6.6,6.6,6.6 \mathrm{~Hz}, 1 \mathrm{H}), 1.49$ (dddd, $J=13.2,6.6,6.6,6.6 \mathrm{~Hz}, 1 \mathrm{H}), 1.43-1.38(\mathrm{~m}, 2 \mathrm{H}) ;{ }^{13} \mathrm{C}-\mathrm{NMR}\left(150 \mathrm{MHz}, \mathrm{CDCl}_{3}\right) ; \delta=170.6,168.5,74.4,52.5$, 52.4, 33.5, 27.6, 25.6, 20.9; IR (thin film) $v_{\max }=3443,3324,3255,2006,2956,2862,1734,1592,1438,1394,1290$, 1213, 1131; HRMS calc. for $\mathrm{C}_{9} \mathrm{H}_{15} \mathrm{NO}_{5}=217.0951$, exp. $=217.0951$.

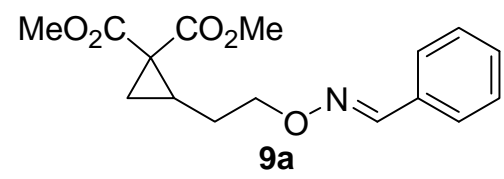

Compound 9a was prepared according to General Procedure A. The amounts of reagents employed were: cyclopropylalkoxylamine $17(0.267 \mathrm{~g}, 1.23 \mathrm{mmol})$, benzaledehyde $(0.143 \mathrm{~g}, 1.35 \mathrm{mmol})$. An 8:1 mixture of trans/cis isomers was observed in the crude reaction mixture by ${ }^{1} \mathrm{H}$ NMR, which were separable to yield trans-oxime ether 9a $(0.285 \mathrm{~g}, 0.93$ mmol, $76 \%)$ and the minor cis-oxime ether $(0.030 \mathrm{~g}, 0.01 \mathrm{mmol}, 8 \%)$ both as colourless oils (85\% overall yield). 9a; ${ }^{1} \mathrm{H}-\mathrm{NMR}\left(600 \mathrm{MHz}, \mathrm{CDCl}_{3}\right) ; \delta=8.07(\mathrm{~s}, 1 \mathrm{H}), 7.57-7.56(\mathrm{~m}, 2 \mathrm{H}), 7.36-7.35(\mathrm{~m}, 3 \mathrm{H}), 4.24(\mathrm{t}, J=6.6 \mathrm{~Hz}, 2 \mathrm{H}), 3.76(\mathrm{~s}$, 3H), $3.71(\mathrm{~s}, 3 \mathrm{H}), 2.06$ (dddd, $\left.J_{1-4}=7.8 \mathrm{~Hz}\right), 1.88(\mathrm{dddd}, J=6.6,6.6,6.6,13.2 \mathrm{~Hz}, 1 \mathrm{H}), 1.64(\mathrm{dddd}, J=6.6,6.6,6.6$, $13.2 \mathrm{~Hz}, 1 \mathrm{H}), 1.47-1.43(\mathrm{~m}, 2 \mathrm{H}) ;{ }^{13} \mathrm{C}-\mathrm{NMR}\left(100 \mathrm{MHz}, \mathrm{CDCl}_{3}\right) ; \delta=170.6,168.5,148.7,132.2,129.7,128.6,126.9$, 72.8, 52.6, 52.5, 33.7, 28.6, 25.5, 21; IR (thin film) $v_{\max }=3027,3003,2953,2878,1728,1437,1333,1291,1267,1213$, 1196, 1131, 1075, 1046, 1028, 949, 917, 758, 694; HRMS calc. for $\mathrm{C}_{16} \mathrm{H}_{19} \mathrm{NO}_{5}=305.1258$, exp. $=305.1263$. 


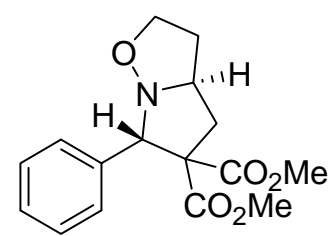

$11 a$

Compound 11a was prepared according to General Procedure B. The amounts of reagents employed were: oxime ether 9a $(0.267 \mathrm{~g}, 0.87 \mathrm{mmol})$ and $\mathrm{Yb}(\mathrm{OTf})_{3}{ }^{\bullet} \mathrm{xH}_{2} \mathrm{O}(0.054 \mathrm{~g}, 0.09 \mathrm{mmol})$. Compound 11a $(0.263 \mathrm{~g}, 0.86 \mathrm{mmol}, 98 \%)$ was obtained as a white solid. $\mathrm{mp}=106-108{ }^{\circ} \mathrm{C} ;{ }^{1} \mathrm{H}-\mathrm{NMR}\left(600 \mathrm{MHz}, \mathrm{CDCl}_{3}\right) ; \delta=7.49-7.48(\mathrm{~m}, 2 \mathrm{H}), 7.30-7.27(\mathrm{~m}, 2 \mathrm{H})$, 7.24-7.21 (m, 1H), $5.02(\mathrm{~s}, 1 \mathrm{H}), 4.20\left(\mathrm{dddd}, J_{1}=4.2,7.8,7.8,7.8 \mathrm{~Hz}\right), 4.06\left(\mathrm{ddd}, J_{1-3}=7.8 \mathrm{~Hz}\right), 3.92(\mathrm{ddd}, J=4.2,8.4$, 8.4 Hz, 1H), $2.99(\mathrm{dd}, J=7.8,13.2 \mathrm{~Hz}, 1 \mathrm{H}), 2.45(\mathrm{dddd}, J=8.4,12.6,12.6,12.6 \mathrm{~Hz}, 1 \mathrm{H}) 2.08(\mathrm{dd}, J=7.8,13.2 \mathrm{~Hz}, 1 \mathrm{H})$, $2.06-2.02(\mathrm{~m}, 1 \mathrm{H}) ;{ }^{13} \mathrm{C}-\mathrm{NMR}\left(100 \mathrm{MHz}, \mathrm{CDCl}_{3}\right) ; \delta=170.7,169.2,137.8,128.0,127.9,127.6,72.4,65.0,62.5,52.8$, $52.1,38.7,36.1$; IR (thin film) $v_{\max }=3033,2980,2886,1734,1449,1438,1257,1179,1068,753$, 701; HRMS calc. for $\mathrm{C}_{16} \mathrm{H}_{19} \mathrm{NO}_{5}=305.1263$, exp. $=305.1263$.
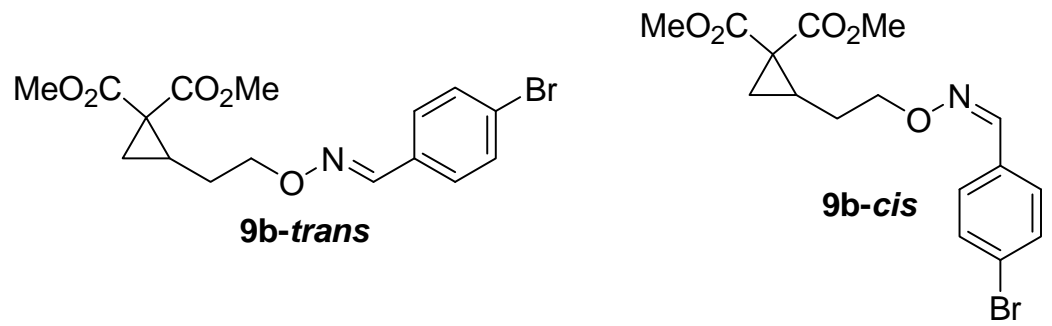

Compounds 9b-trans and 9b-cis were prepared according to General Procedure A. The amounts of reagents employed were: cyclopropyl-alkoxylamine 17 (0.250 g, $1.15 \mathrm{mmol})$, p-bromo-benzaledehyde $(0.234 \mathrm{~g}, 1.26 \mathrm{mmol})$. An 8 : 1 mixture of trans to cis isomers were observed in the crude reaction mixture by ${ }^{1} \mathrm{H}$ NMR, which were separable to yield oxime ether 9b-trans (0.362 g, $0.95 \mathrm{mmol}, 82 \%)$, and oxime ether $9 \mathrm{~b}$-cis $(0.060 \mathrm{~g}, 0.02 \mathrm{mmol}, 13 \%)$, both as colourless oils (95\% overall yield).

9b-trans; ${ }^{1} \mathrm{H}-\mathrm{NMR}\left(600 \mathrm{MHz}, \mathrm{CDCl}_{3}\right) ; \delta=7.74(\mathrm{~d}, J=7.8 \mathrm{~Hz}, 1 \mathrm{H}), 7.54(\mathrm{~d}, J=7.8 \mathrm{~Hz}, 1 \mathrm{H}), 7.25(\mathrm{~s}, 1 \mathrm{H}), 4.29(\mathrm{ddd}, J=$ 3.0, 6.0, $6.0 \mathrm{~Hz}, 1 \mathrm{H}), 2.02\left(\mathrm{dddd}, J_{1-4}=8.4 \mathrm{~Hz}, 1 \mathrm{H}\right), 1.92(\mathrm{dddd}, J=5.4,5.4,5.4,15 \mathrm{~Hz}, 1 \mathrm{H}), 1.66\left(\mathrm{dddd}, J_{1-4}=7.2 \mathrm{~Hz}\right.$, 1H), 1.47-1.42 (m, 2H); ${ }^{13} \mathrm{C}-\mathrm{NMR}\left(100 \mathrm{MHz}, \mathrm{CDCl}_{3}\right) ; \delta=170.5,168.5,144.8,132.2,131.6,129.3,124.3,73.8,52.6$, $52.5,33.5,28.5,25.4,21.0$; IR (thin film) $v_{\max }=3006,2953,2883,2850,1734,1586,1485,1437,1399,1332,1292$, 1214, 1131, 1072, 1045, 1011, 907, 820, 733, 709, 570; HRMS calc. for $\mathrm{C}_{16} \mathrm{H}_{18} \mathrm{BrNO}_{5}=383.0360$, exp. $=383.0368$. 
9b-cis; ${ }^{1} \mathrm{H}-\mathrm{NMR}\left(600 \mathrm{MHz}, \mathrm{CDCl}_{3}\right) ; \delta=8.00(\mathrm{~s}, 1 \mathrm{H}), 7.49(\mathrm{~d}, J=7.8 \mathrm{~Hz}, 2 \mathrm{H}), 7.43(\mathrm{~d}, J=7.8 \mathrm{~Hz}, 2 \mathrm{H}), 4.22$, (t, $J=6.6$ $\mathrm{Hz}, 2 \mathrm{H}), 3.76(\mathrm{~s}, 3 \mathrm{H}), 3.71(\mathrm{~d}, 3 \mathrm{H}), 2.04$ (quin, $J=7.8 \mathrm{~Hz}, 1 \mathrm{H}), 1.86$ (dddd, $\left.J_{1}=13.2, J_{2-4}=6.6 \mathrm{~Hz}, 1 \mathrm{H}\right), 1.63\left(\mathrm{dddd}, J_{1}=\right.$ $\left.13.2, J_{2-4}=6.6 \mathrm{~Hz}, 1 \mathrm{H}\right), 1.47-1.42(\mathrm{~m}, 2 \mathrm{H}) ;{ }^{13} \mathrm{C}-\mathrm{NMR}\left(100 \mathrm{MHz}, \mathrm{CDCl}_{3}\right) ; \delta=170.6,168.5,147.5,131.9,131.2,128.3$, 123.9, 73.0, 52.6, 52.5, 33.7, 28.5, 25.4, 21.0; IR (thin film) $v_{\max }=2953,1725,1489,1437,1399,1291,1214,1131$, 1070, 1010, 821; HRMS calc. for $\mathrm{C}_{16} \mathrm{H}_{18} \mathrm{BrNO}_{5}=383.0360$, exp. $=383.0368$.

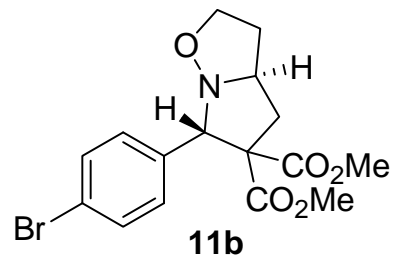

Compound 11b was prepared according to General Procedure B. The amounts of reagents employed were: oxime ether 9b-trans $(0.148 \mathrm{~g}, 0.39 \mathrm{mmol})$ and $\mathrm{Yb}(\mathrm{OTf})_{3} \bullet \mathrm{xH}_{2} \mathrm{O}(0.024 \mathrm{~g}, 0.04 \mathrm{mmol})$. Compound $11 \mathrm{~b}(0.148 \mathrm{~g}, 0.39 \mathrm{mmol}$, quantitative yield) was obtained as a white solid. ${ }^{1} \mathrm{H}-\mathrm{NMR}\left(600 \mathrm{MHz}, \mathrm{CDCl}_{3}\right) ; \delta=7.43-7.40(\mathrm{~m}, 4 \mathrm{H}), 4.91(\mathrm{~s}, 1 \mathrm{H}), 4.18$ (dddd, $J=4.2,7.2,7.2,7.2 \mathrm{~Hz}, 1 \mathrm{H}), 2.03\left(\mathrm{ddd}, J_{1-3}=8.4 \mathrm{~Hz}, 1 \mathrm{H}\right), 3.93$ (ddd, $\left.J=4.2,8.4,8.4 \mathrm{~Hz}\right), 3.74(\mathrm{~s}, 3 \mathrm{H}), 3.28$ (s, 3H), $2.98(\mathrm{dd}, J=7.2,13.2 \mathrm{~Hz}, 1 \mathrm{H}), 2.44(\mathrm{dddd}, J=8.4,8.4,8.4,13.2 \mathrm{~Hz}, 1 \mathrm{H}), 2.05(\mathrm{dd}, J=7.2,13.2 \mathrm{~Hz}, 1 \mathrm{H}), 2.04-$ $2.00(\mathrm{~m}, 1 \mathrm{H}) ;{ }^{13} \mathrm{C}-\mathrm{NMR}\left(100 \mathrm{MHz}, \mathrm{CDCl}_{3}\right) ; \delta=170.5,169.0,137.0,131.0,130.0,121.7,71.6,65.0,64.6,62.1,52.9$, $52.2,38.8,36.2$; IR (thin film) $v_{\max }=2953,2883,1734,1488,1435,1278,1215,1178,1120,1102,1071,984,914,856$, 733, 713; HRMS calc. for $\mathrm{C}_{16} \mathrm{H}_{18} \mathrm{BrNO}_{5}=383.0364$, exp. $=383.0368 . ; \mathrm{mp}(+/-)-\mathbf{1 1 b}=111-114{ }^{\circ} \mathrm{C} ;(2 S, 5 R)-\mathbf{1 1 b}$ was a colourless oil; $(2 S, 5 R)-11 b,[\alpha]_{\mathrm{D}}^{22}=+86.00^{\circ}\left(\mathrm{c} 8.1, \mathrm{CH}_{2} \mathrm{Cl}_{2}\right)$; HPLC $($ Chiracel OD-H, $7 \%$ iPrOH/hexanes, 1.0 $\mathrm{mL} / \mathrm{min}),(2 R, 5 S ; 2 S, 5 R) \mathrm{RT}=9.60,13.13 \mathrm{~min},(2 S, 5 R) \mathrm{RT}=13.03 \mathrm{~min} ;$ ee $=>99 \%$.

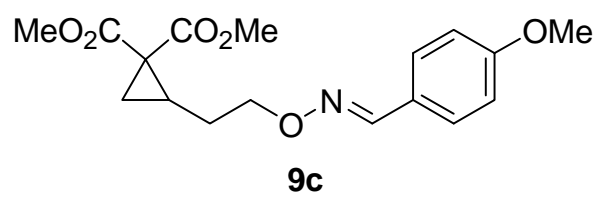

Compound 9c was prepared according to General Procedure A. The amounts of reagents employed were: cyclopropylalkoxylamine $17(0.205 \mathrm{~g}, 0.95 \mathrm{mmol})$, anisaldehyde $(0.142 \mathrm{~g}, 1.05 \mathrm{mmol})$. A $12: 1 \mathrm{mixture}$ of trans to cis isomers was observed in the crude reaction mixture by ${ }^{1} \mathrm{H}$ NMR, which were separable to yield trans-oxime ether 9c-trans $(0.291 \mathrm{~g}$, $0.87 \mathrm{mmol}, 92 \%)$ and the minor cis-oxime ether $(0.025 \mathrm{~g}, 0.08 \mathrm{mmol}, 8 \%)$ both as colourless oils (quantitative overall 
yield). 9c; ${ }^{1} \mathrm{H}-\mathrm{NMR}\left(600 \mathrm{MHz}, \mathrm{CDCl}_{3}\right) ; \delta=8.01(\mathrm{~s}, 1 \mathrm{H}), 7.50(\mathrm{~d}, J=9 \mathrm{~Hz}, 2 \mathrm{H}), 6.88(\mathrm{~d}, J=9 \mathrm{~Hz}, 2 \mathrm{H}), 4.20(\mathrm{t}, J=6.6$ $\mathrm{Hz}, 2 \mathrm{H}), 3.82(\mathrm{~s}, 3 \mathrm{H}), 3.76(\mathrm{~s}, 3 \mathrm{H}), 3.71(\mathrm{~s}, 3 \mathrm{H}), 2.08-2.02(\mathrm{~m}, 1 \mathrm{H}), 1.87$ (dddd, $J=6.0,6.0,6.0,7.8 \mathrm{~Hz}, 1 \mathrm{H}), 1.62$ (dddd, $J=7.2,7.2,7.2,14.4 \mathrm{~Hz}, 1 \mathrm{H}), 1.47-1.42(\mathrm{~m}, 2 \mathrm{H}) ;{ }^{13} \mathrm{C}-\mathrm{NMR}\left(100 \mathrm{MHz}, \mathrm{CDCl}_{3}\right) ; \delta=170.7,128.6,160.9,148.3,128.4$, $124.9,114.0,72.6,55.3,52.6,52.5,33.7,28.6,25.6,21.0 ;$ IR (thin film) $v_{\max }=3114,2954,2877,1841,1727,1308$, $1514,1438,1335,1300,1252,1214,1076,952,812,833,812$; HRMS calc. for $\mathrm{C}_{17} \mathrm{H}_{21} \mathrm{NO}_{6}=335.1367$, exp. $=335.1369$.

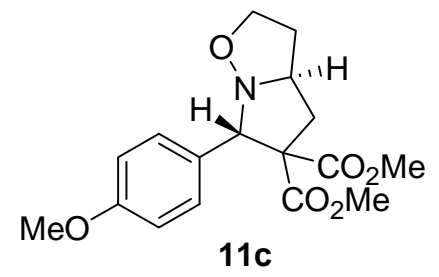

Compound 11c was prepared according to General Procedure B. The amounts of reagents employed were: oxime ether 9c $(0.264 \mathrm{~g}, 0.79 \mathrm{mmol}), \mathrm{Yb}(\mathrm{OTf})_{3} \bullet \mathrm{xH}_{2} \mathrm{O}(0.049 \mathrm{~g}, 0.08 \mathrm{mmol})$. Compound 11c $(0.264 \mathrm{~g}, 0.79 \mathrm{mmol}$, quantitative yield $)$ was obtained as a white solid: $\mathrm{mp}=88-90^{\circ} \mathrm{C} ;{ }^{1} \mathrm{H}-\mathrm{NMR}\left(600 \mathrm{MHz}, \mathrm{CDCl}_{3}\right) ; \delta=7.41(\mathrm{~d}, J=8.4 \mathrm{~Hz}, 2 \mathrm{H}), 6.83(\mathrm{~d}, J=$ $8.4 \mathrm{~Hz}, 2 \mathrm{H}), 4.94(\mathrm{~s}, 1 \mathrm{H}), 4.17$ (dddd, $J=4.2,7.8,7.8,7.8 \mathrm{~Hz}, 1 \mathrm{H}), 4.05$ (ddd, $\left.J_{1-3}=7.8 \mathrm{~Hz}, 1 \mathrm{H}\right), 3.91(\mathrm{ddd}, J=4.2,8.4$, $8.4 \mathrm{~Hz}, 1 \mathrm{H}), 3.77$ (s, 3H), $3.74(\mathrm{~s}, 3 \mathrm{H}), 3.28(\mathrm{~s}, 3 \mathrm{H}), 2.98(\mathrm{dd}, J=6.6,13.2 \mathrm{~Hz}, 1 \mathrm{H}), 2.44$ (dddd, $J=8.4,8.4,8.4,12.6$ $\left.\mathrm{Hz}, 1 \mathrm{H}), 2.06(\mathrm{dd}, J=6.6,13.2 \mathrm{~Hz}, 1 \mathrm{H}), 2.02(\mathrm{dddd}, J=4.2,4.2,7.2,11.4 \mathrm{~Hz}, 1 \mathrm{H}) ;{ }^{13} \mathrm{C}-\mathrm{NMR}\left(100 \mathrm{MHz}^{\mathrm{CDCl}}\right)_{3}\right) \delta=$ $170.8,169.3,159.1,129.8,129.1,113.3,71.9,65.0,64.8,62.2,55.2,52.8,52.2,38.6,36.2 ;$ IR (thin film) $v_{\max }=2907$, $2840,1733,1613,1513,1457,1437,1302,1275,1249,1215,1207,1176,1119,1069,1033,915,843,822,798,736$; HRMS calc. for $\mathrm{C}_{17} \mathrm{H}_{21} \mathrm{NO}_{6}=3335.1367$, exp. $=335.1369$.

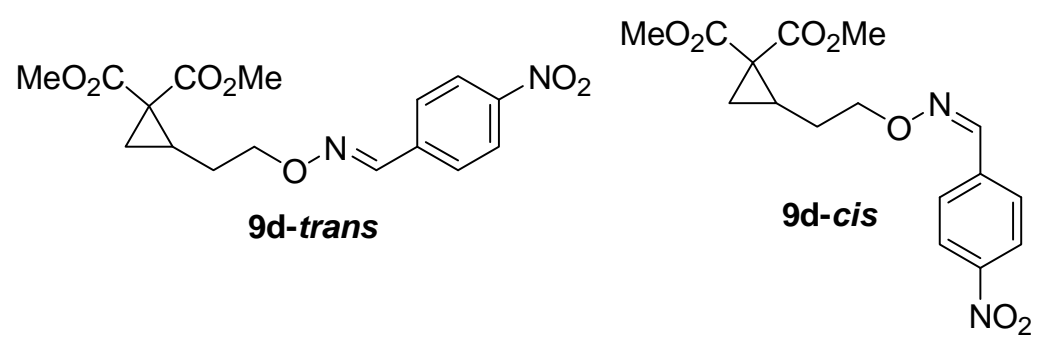

Compounds 9d-trans and 9d-cis were prepared according to General Procedure A. The amounts of reagents employed were: cyclopropyl-alkoxylamine $17(0.227 \mathrm{~g}, 1.05 \mathrm{mmol})$, p-nitro-benzaledehyde $(0.174 \mathrm{~g}, 1.15 \mathrm{mmol})$. A $3: 1 \mathrm{mixture}$ of trans to cis isomers was observed in the crude reaction mixture by ${ }^{1} \mathrm{H}$ NMR, which were separable to yield 9d-trans $(0.263 \mathrm{~g}, 0.75 \mathrm{mmol}, 72 \%)$ and 9d-cis (0.094 g, $0.27 \mathrm{mmol}, 26 \%)$ both as colourless oils (98 \% overall yield). 
9d-trans: ${ }^{1} \mathrm{H}-\mathrm{NMR}\left(600 \mathrm{MHz}, \mathrm{CDCl}_{3}\right) ; \delta=8.22(\mathrm{~d}, J=8.4 \mathrm{~Hz}, 2 \mathrm{H}), 8.11(\mathrm{~s}, 1 \mathrm{H}), 7.73(\mathrm{~d}, J=8.4 \mathrm{~Hz}), 4.29(\mathrm{t}, J=6.0 \mathrm{~Hz}$, 2H), $3.77(\mathrm{~s}, 3 \mathrm{H}), 3.72(\mathrm{~s}, 3 \mathrm{H}), 2.05\left(\mathrm{dddd}, J_{1-4}=7.8 \mathrm{~Hz}, 1 \mathrm{H}\right), 1.89$ (dddd, $\left.J=6.6,6.6,6.6,14.4 \mathrm{~Hz}, 1 \mathrm{H}\right), 1.67(\mathrm{dddd}, J=$ 6.6, 6.6, 6.6, 14.4 Hz), 1.48-1.43 (m, 2H); ${ }^{13} \mathrm{C}-\mathrm{NMR}\left(100 \mathrm{MHz}, \mathrm{CDCl}_{3}\right) ; \delta=170.6,168.5,148.3,146.5,138.4,127.5$, 124.0, 77.2, 73.7, 52.7, 52.6, 33.7, 28.5, 25.3, 21.0; IR (thin film) $v_{\max }=3009,2881,2850,1728,1599,1522,1437,1345$, 1294, 1214, 1131, 1042, 955, 832, 751, 692; HRMS calc. for $\mathrm{C}_{16} \mathrm{H}_{18} \mathrm{~N}_{2} \mathrm{O}_{7}=350.1105$, exp. $=350.1114$.

9d-cis: ${ }^{1} \mathrm{H}-\mathrm{NMR}\left(600 \mathrm{MHz}, \mathrm{CDCl}_{3}\right) ; \delta=8.24(\mathrm{~d}, J=7.8 \mathrm{~Hz}, 2 \mathrm{H}), 8.02(\mathrm{~d}, J=7.8,2 \mathrm{H}), 7.40(\mathrm{~s}, 1 \mathrm{H}), 4.33(\mathrm{ddd}, J=1.8$, 6.0, $6.0 \mathrm{~Hz}, 1 \mathrm{H}), 3.76(\mathrm{~d}, J=1.8 \mathrm{~Hz}, 3 \mathrm{H}), 3.71(\mathrm{~d}, J=1.8 \mathrm{~Hz}, 3 \mathrm{H}), 2.01$ (dddd, $\left.J_{1-4}=7.2 \mathrm{~Hz}, 1 \mathrm{H}\right), 1.91(\mathrm{dddd}, J=6.0$, 6.0, 6.0, $13.8 \mathrm{~Hz}, 1 \mathrm{H}), 1.69$ (dddd, $J=7.2,7.2,7.2,13.8 \mathrm{~Hz}, 1 \mathrm{H}), 1.46-91.41(\mathrm{~m}, 2 \mathrm{H}) ;{ }^{13} \mathrm{C}-\mathrm{NMR}\left(100 \mathrm{MHz}, \mathrm{CDCl}_{3}\right) ; \delta=$ $170.5,168.5,147.8,143.6,135.7,131.4,123.6,74.3,52.7,52.6,33.5,28.4,25.2,21.0 ;$ IR (thin film) $v_{\max }=3074,3006$, 2954, 2886, 1724, 1598, 1522, 1437, 1346, 1293, 1214, 1194, 1132, 1044, 914, 854, 912, 792, 753, 734; HRMS calc. for $\mathrm{C}_{16} \mathrm{H}_{18} \mathrm{~N}_{2} \mathrm{O}_{7}=350.1101$, exp. $=350.1114$.

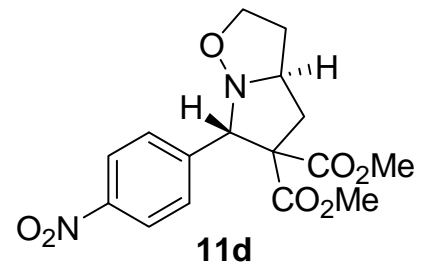

Compound 11d was prepared according to General Procedure B. The amounts of reagents employed were: oxime ether 9d-trans $(0.267 \mathrm{~g}, 0.76 \mathrm{mmol}), \mathrm{Yb}(\mathrm{OTf})_{3} \bullet \mathrm{xH}_{2} \mathrm{O}(0.047 \mathrm{~g}, 0.08 \mathrm{mmol})$. Compound 11d (0.267 g, $0.76 \mathrm{mmol}$, quantitative yield) was obtained as a colourless oil. ${ }^{1} \mathrm{H}-\mathrm{NMR}\left(600 \mathrm{MHz}, \mathrm{CDCl}_{3}\right) ; \delta=8.16(\mathrm{~d}, J=9 \mathrm{~Hz}, 2 \mathrm{H}), 7.75(\mathrm{~d}, J=9 \mathrm{~Hz}, 2 \mathrm{H})$, 4.23 (dddd, $J=3.6,7.8,7.8,7.8 \mathrm{~Hz}, 1 \mathrm{H}), 4.04(\mathrm{ddd}, J=7.2,9.0,9.0 \mathrm{~Hz}, 1 \mathrm{H}), 3.96(\mathrm{ddd}, J=3.6,7.8,7.8 \mathrm{~Hz}, 1 \mathrm{H}), 3.76$ (s, 3H), $3.28(\mathrm{~s}, 3 \mathrm{H}), 3.01(\mathrm{dd}, J=7.2,13.2 \mathrm{~Hz}, 1 \mathrm{H}), 2.47$ (dddd, $J=12.0,14.4,14.4,14.4 \mathrm{~Hz}, 1 \mathrm{H}), 2.07(\mathrm{dd}, J=13.8$, $12.6 \mathrm{~Hz}, 1 \mathrm{H}), 2.04(\mathrm{dddd}, J=3.6,3.6,7.2,12 \mathrm{~Hz}) ;{ }^{13} \mathrm{C}-\mathrm{NMR}\left(100 \mathrm{MHz}, \mathrm{CDCl}_{3}\right) ; \delta=170.2,168.6,147.5,145.7,129.1$, 123.0, 71.6, 65.0, 64.8, 62.0, 53.1, 52.3, 39.0, 36.3; IR (thin film) $v_{\max }=2981,2883,1734,1605,1522,1493,1349,1316$, 1277, 1259, 1207, 1179, 1122, 915, 860, 843, 749, 735, 697; HRMS calc.for $\mathrm{C}_{16} \mathrm{H}_{18} \mathrm{~N}_{2} \mathrm{O}_{7}=350.1113$, exp. $=350.1114$.

The oxime ether 9d-cis $(0.063 \mathrm{~g}, 0.18 \mathrm{mmol})$ was also cyclized according to General Procedure B. Compound 21d (0.047 $\mathrm{g}, 0.13 \mathrm{mmol}, 74 \%)$ was obtained as a colourless oil (vide infra for spectral data). 


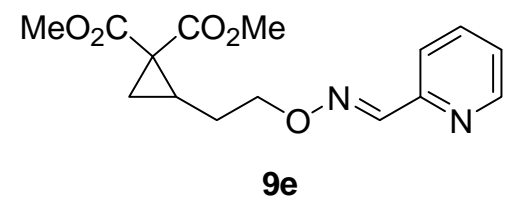

Compound 9e was prepared according to General Procedure A. The amounts of reagents employed were: cyclopropylalkoxylamine $17(0.255 \mathrm{~g}, 1.17 \mathrm{mmol})$, 2-pyridinecarboxaldehyde $(0.138 \mathrm{~g}, 1.29 \mathrm{mmol})$. A $14: 1$ mixture of trans to cis isomers was observed in the crude reaction mixture by ${ }^{1} \mathrm{H}$ NMR, which were separable to yield trans-oxime ether 9e (0.304 g, $0.99 \mathrm{mmol}, 84 \%)$ and the minor cis-oxime ether $(0.021 \mathrm{~g}, 0.07 \mathrm{mmol}, 6 \%)$ both as colourless oils $(90 \%$ overall yield). 9e; ${ }^{1} \mathrm{H}-\mathrm{NMR}\left(600 \mathrm{MHz}, \mathrm{CDCl}_{3}\right) ; \delta=8.57(\mathrm{~d}, J=4.8 \mathrm{~Hz}, 1 \mathrm{H}), 8.13(\mathrm{~s}, 1 \mathrm{H}), 7.74(\mathrm{~d}, J=7.2 \mathrm{~Hz}, 1 \mathrm{H}), 7.66$ $(\mathrm{dt}, J=1.2,7.2 \mathrm{~Hz}, 1 \mathrm{H}), 7.26-7.21(\mathrm{~m}, 1 \mathrm{H}), 4.26(\mathrm{t}, J=6.6 \mathrm{~Hz}, 2 \mathrm{H}), 3.74(\mathrm{~s}, 3 \mathrm{H}), 3.68(\mathrm{~s}, 3 \mathrm{H}), 2.03\left(\mathrm{dddd}, J_{1-4}=7.2 \mathrm{~Hz}\right.$ 1H), 1.87 (dddd, $J=6.6,6.6,6.6,14.4 \mathrm{~Hz}, 1 \mathrm{H}), 1.63(\mathrm{dddd}, J=6.6,6.6,6.6,14.4 \mathrm{~Hz}, 1 \mathrm{H}), 1.45-1.40(\mathrm{~m}, 2 \mathrm{H})$; ${ }^{13} \mathrm{C}-\mathrm{NMR}$ $\left(100 \mathrm{MHz}, \mathrm{CDCl}_{3}\right) ; \delta=170.4,128.4,151.5,149.5,149.2,136.3,123.8,120.8,73.2,52.4,52.4,33.5,28.4,25.2,20.8 ; \mathrm{IR}$ (thin film) $v_{\max }=3004,2953,1728,1586,1469,1457,1334,1291,1214,1196,1131,1074,1045,993,950,921,778$, 744; HRMS calc. for $\mathrm{C}_{15} \mathrm{H}_{18} \mathrm{~N}_{2} \mathrm{O}_{5}=306.1210$, exp. $=306.1216$.

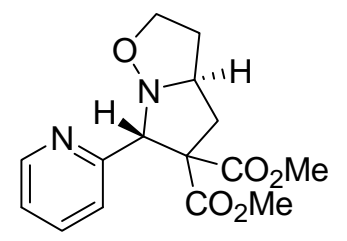

$11 e$

Compound 11e was prepared according to General Procedure B. The amounts of reagents employed were: oxime ether 9e $(0.209 \mathrm{~g}, 0.68 \mathrm{mmol})$ and $\mathrm{Yb}(\mathrm{OTf})_{3} \bullet \mathrm{xH}_{2} \mathrm{O}(0.021 \mathrm{~g}, 0.03 \mathrm{mmol})$. Compound 11e $(0.209 \mathrm{~g}, 0.68$ mmol, quantitative yield) was obtained as a white solid. $\mathrm{mp}=74-76{ }^{\circ} \mathrm{C} ;{ }^{1} \mathrm{H}-\mathrm{NMR}\left(600 \mathrm{MHz}, \mathrm{CDCl}_{3}\right) ; \delta=8.50(\mathrm{dd}, J=1.2,4.8 \mathrm{~Hz}, 1 \mathrm{H})$, $7.64(\mathrm{ddd}, J=1.8,7.8,7.8 \mathrm{~Hz}, 1 \mathrm{H}), 7.50(\mathrm{~d}, J=7.8 \mathrm{~Hz}, 1 \mathrm{H}), 7.13(\mathrm{ddd}, J=1.2,4.8,7.8 \mathrm{~Hz}, 1 \mathrm{H}), 5.31(\mathrm{~s}, 1 \mathrm{H}), 4.17$ (dddd, $J=2.4,7.8,7.8,7.8 \mathrm{~Hz}, 1 \mathrm{H}), 4.00\left(\mathrm{ddd}, J_{1-3}=7.8 \mathrm{~Hz}, 1 \mathrm{H}\right), 3.87$ (ddd, $\left.J=4.8,8.4,8.4 \mathrm{~Hz}, 1 \mathrm{H}\right), 3.77$ (s, $\left.3 \mathrm{H}\right), 3.36$ (s, 3H), 3.10 (dd, $J=7.8,13.2 \mathrm{~Hz}, 1 \mathrm{H}), 2.45$ (dddd, $J=7.8,7.8,7.8,12.6 \mathrm{~Hz}, 1 \mathrm{H}), 2.33(\mathrm{dd}, J=7.2,13.2 \mathrm{~Hz}, 1 \mathrm{H}), 2.13$ (dddd, $J=3.0,4.8,7.8,12.6 \mathrm{~Hz}, 1 \mathrm{H}) ;{ }^{13} \mathrm{C}-\mathrm{NMR}\left(100 \mathrm{MHz}, \mathrm{CDCl}_{3}\right) ; \delta=170.5,169.3,157.4,148.8,136.2,123.4,122.4$, $75.8,65.0,63.2,53.1,52.3,39.1,36.2$; IR (thin film) $v_{\max }=2955,2881,1735,1592,1572,1473,1456,1436,1259,1227$, 1208, 1175, 1122, 1010, 999, 915, 858, 770, 750; HRMS calc. for $\mathrm{C}_{15} \mathrm{H}_{18} \mathrm{~N}_{2} \mathrm{O}_{5}=306.1216$, exp. $=306.1216$. 


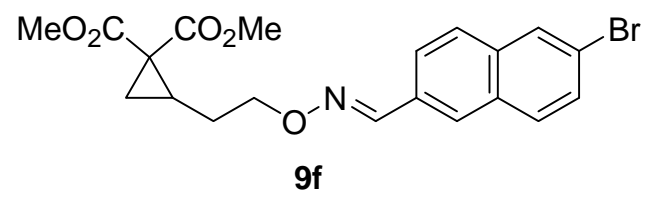

Compound 9f was prepared according to General Procedure A. The amounts of reagents employed were: cyclopropylalkoxylamine 17 (0.108 g, $0.50 \mathrm{mmol})$, 6-bromo-napthalene-2-carboxaldehyde $(0.129 \mathrm{~g}, 0.55 \mathrm{mmol})$. A $49: 1$ mixture of trans to cis isomers was observed in the crude reaction mixture by ${ }^{1} \mathrm{H} \mathrm{NMR}$, which were separable and upon purification by flash column chromatography yielded trans-oxime ether $\mathbf{9 f}(0.197 \mathrm{~g}, 0.45 \mathrm{mmol}, 91 \%)$ as a colourless oil. 9 9f; ${ }^{1} \mathrm{H}-$ $\operatorname{NMR}\left(400 \mathrm{MHz}, \mathrm{CDCl}_{3}\right) ; \delta=8.17(\mathrm{~s}, 1 \mathrm{H}), 7.95(\mathrm{~d}, J=2.0 \mathrm{~Hz}, 1 \mathrm{H}), 7.84(\mathrm{dd}, J=9.2 \mathrm{~Hz}, 1.6 \mathrm{~Hz}, 1 \mathrm{H}), 7.79(\mathrm{~s}, 1 \mathrm{H}), 7.69$ $(\mathrm{d}, J=7.6 \mathrm{~Hz}, 1 \mathrm{H}), 7.66(\mathrm{~d}, J-8.8 \mathrm{~Hz}, 1 \mathrm{H}), 7.53(\mathrm{dd}, J=8.4,2.0 \mathrm{~Hz}, 1 \mathrm{H}), 4.28(\mathrm{t}, J=6.4 \mathrm{~Hz}, 2 \mathrm{H}), 3.77(\mathrm{~s}, 3 \mathrm{H}), 3.71(\mathrm{~s}$, 3H), $2.12-2.05(\mathrm{~m}, 1 \mathrm{H}), 1.91(\mathrm{dddd}, J=14.0,6.0,6.0,6.0 \mathrm{~Hz}, 1 \mathrm{H}), 1.72-1.63(\mathrm{~m}, 1 \mathrm{H}), 1.50-1.44(\mathrm{~m}, 2 \mathrm{H}) ;{ }^{13} \mathrm{C}-\mathrm{NMR}$ $\left(100 \mathrm{MHz}, \mathrm{CDCl}_{3}\right) ; \delta=170.6,168.5,148.3,134.9,131.5,130.3,129.9,129.7,127.9,127.5,124.0,120.8,73.0,52.5$ (2C), 33.6, 28.5, 25.5, 21.0; IR (thin film) $v_{\max }=2952,1727,1437,1330,1291,1267,1213,1131,1063,1045,944,900$, 882, 806; HRMS calc. for $\mathrm{C}_{20} \mathrm{H}_{20} \mathrm{BrNO}_{5}=433.0536$, exp. $=433.0525$.

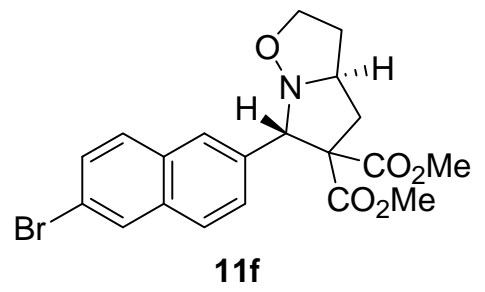

Compound 11f was prepared according to General Procedure B. The amounts of reagents employed were: oxime ether 9f $(0.197 \mathrm{~g}, 0.45 \mathrm{mmol})$ and $\mathrm{Yb}(\mathrm{OTf})_{3} \bullet \mathrm{xH}_{2} \mathrm{O}(0.014 \mathrm{~g}, 0.02 \mathrm{mmol})$. Compound 11f $(0.195 \mathrm{~g}, 0.45 \mathrm{mmol}, 99 \%)$ was obtained as a white solid. $(2 S, 5 R)-\mathbf{1 1 f}, \mathrm{mp}=146-147{ }^{\circ} \mathrm{C} ;(+/-)-\mathbf{1 1 f}, \mathrm{mp}=121-122{ }^{\circ} \mathrm{C}(\mathrm{MeOH}) ;{ }^{1} \mathrm{H}-\mathrm{NMR}(600 \mathrm{MHz}$, $\left.\mathrm{CDCl}_{3}\right) ; \delta=7.99(\mathrm{~s}, 1 \mathrm{H}), 7.94(\mathrm{~d}, J=1.2 \mathrm{~Hz}, 1 \mathrm{H}), 7.69(\mathrm{~d}, \mathrm{H}=7.8 \mathrm{~Hz}, 1 \mathrm{H}), 7.68(\mathrm{~d}, J=7.8 \mathrm{~Hz}, 1 \mathrm{H}), 7.64(\mathrm{dd}, J=15.0$, $1.8 \mathrm{~Hz}, 1 \mathrm{H}), 7.50(\mathrm{dd}, J=9.0,1.8 \mathrm{~Hz}, 1 \mathrm{H}), 5.13(\mathrm{~s}, 1 \mathrm{H}), 4.27(\mathrm{ddd}, J=7.8,7.8,7.8,3.6 \mathrm{~Hz}, 1 \mathrm{H}), 4.09$ (ddd, $J=8.4,8.4$, $7.2 \mathrm{~Hz}, 1 \mathrm{H}), 3.96$ (ddd, $J=8.4,8.4,4.2 \mathrm{~Hz}, 1 \mathrm{H}), 3.76(\mathrm{~s}, 3 \mathrm{H}), 3.11(\mathrm{~s}, 3 \mathrm{H}), 3.04$ (dd, $J=12.6,7.8 \mathrm{H}, 1 \mathrm{H}), 2.48$ (dddd, $J$ $=12.0,8.4,8.4,8.4 \mathrm{~Hz}, 1 \mathrm{H}), 2.11(\mathrm{dd}, J=13.2,7.8 \mathrm{~Hz}, 1 \mathrm{H}), 2.07(\mathrm{dddd}, J=12.0,7.2,4.2,4.2 \mathrm{~Hz}, 1 \mathrm{H}) ;{ }^{13} \mathrm{C}-\mathrm{NMR}(100$ $\left.\mathrm{MHz}, \mathrm{CDCl}_{3}\right) ; \delta=170.6,168.1,136.1,134.1,131.5,129.8,129.5,129.1,127.1,127.0,126.4,119.7,72.3,65.1,64.9$ $62.3,52.9,52.1,38.8,36.3$; IR (thin film) $v_{\max }=2975,2953,2885,2829,1733,1436,1277,1256,1213,1133,1064,883$, 812, 736; HRMS calc. for $\mathrm{C}_{20} \mathrm{H}_{20} \mathrm{BrNO}_{5}=433.0523$, exp. $=433.0525 .(2 S, 5 R)-11 f,[\alpha]_{\mathrm{D}}^{22}=+88.4^{\circ}\left(\mathrm{c} 4.1, \mathrm{CH}_{2} \mathrm{Cl}_{2}\right)$; HPLC on column purified material prior to recrystallization: (Chiracel OD, $10 \% \mathrm{iPrOH} / \mathrm{hexanes}, 1.0 \mathrm{ml} / \mathrm{min}),(2 R, 5 S$; 

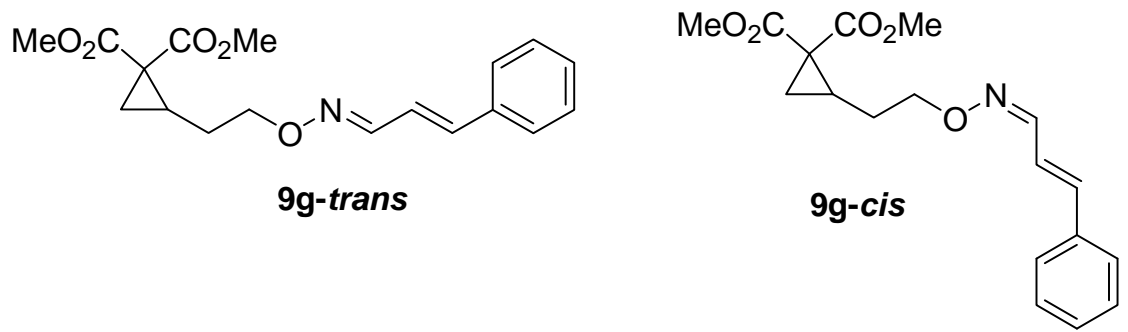

Compounds 9g-trans and 9g-cis were prepared according to General Procedure A. The amounts of reagents employed were: $0.262 \mathrm{~g}(1.21 \mathrm{mmol})$ of cyclopropane $17,0.175 \mathrm{~g}(1.33 \mathrm{mmol})$ of trans-cinnamaldehyde. A $2: 1 \mathrm{mixture}$ of trans to cis isomers was observed in the crude reaction mixture by ${ }^{1} \mathrm{H}$ NMR, which were separable to yield oxime ether 9gtrans $(0.175 \mathrm{~g}, 0.53 \mathrm{mmol}, 44 \%)$ and oxime ether $9 \mathrm{~g}$-cis $(0.088 \mathrm{~g}, 0.26 \mathrm{mmol}, 22 \%)$ both as colourless oils (66 \% overall yield).

9g-trans: ${ }^{1} \mathrm{H}-\mathrm{NMR}\left(600 \mathrm{MHz}, \mathrm{CDCl}_{3}\right) ; \delta=7.88-7.86(\mathrm{~m}, 1 \mathrm{H}), 7.45-7.43(\mathrm{~m}, 2 \mathrm{H}), 7.36-7.33(\mathrm{~m}, 2 \mathrm{H}), 7.30-7.26(\mathrm{~m}, 1 \mathrm{H})$, 6.83-6.77 (m, 2H), $4.18(\mathrm{t}, J=6.6 \mathrm{~Hz}, 2 \mathrm{H}), 3.77(\mathrm{~s}, 3 \mathrm{H}), 3.72(\mathrm{~s}, 3 \mathrm{H}), 2.06-2.00(\mathrm{~m}, 1 \mathrm{H}), 1.86(\mathrm{dddd}, J=6.6,6.6,6.6$, 14.4 Hz, 1H), 1.63-1.57 (m, 1H), 1.47-1.42 (m, 2H); ${ }^{13} \mathrm{C}-\mathrm{NMR}\left(100 \mathrm{MHz}, \mathrm{CDCl}_{3}\right) ; \delta=170.7,168.5,150.6,138.4,136.0$, $128.8,126.9,121.9,72.8,52.6,52.5,33.7,28.5,25.4,21.0$; IR (thin film) $v_{\max }=3003,1726,1449,1332,1291,1266$, 1213, 1130, 1074, 1041, 974, 944, 906, 751, 692; HRMS calc. for $\mathrm{C}_{18} \mathrm{H}_{21} \mathrm{NO}_{5}=331.1414$, exp. $=331.1420$.

9g-cis: ${ }^{1} \mathrm{H}-\mathrm{NMR}\left(600 \mathrm{MHz}, \mathrm{CDCl}_{3}\right) ; \delta=7.51(\mathrm{~d}, 2 \mathrm{H}, J=7.2 \mathrm{~Hz}), 7.38-7.30(\mathrm{~m}, 4 \mathrm{H}), 7.18(\mathrm{~d}, 1 \mathrm{H}, J=9.6 \mathrm{~Hz}), 6.83(\mathrm{~d}$, $1 \mathrm{H}, J=16.2 \mathrm{~Hz}), 4.22(\mathrm{ddd}, 2 \mathrm{H}, J=6.0,6.0,1.8 \mathrm{~Hz}), 3.77(\mathrm{~s}, 3 \mathrm{H}), 3.73(\mathrm{~s}, 3 \mathrm{H}), 2.04\left(\mathrm{dddd}, 1 \mathrm{H}, J_{1-4}=7.8 \mathrm{~Hz}\right), 1.90$ (dddd, $1 \mathrm{H}, J=13.2,6.6,6.6,6.6 \mathrm{~Hz}), 1.62(\mathrm{dddd}, 1 \mathrm{H}, J=13.8,6.6,6.6,6.6 \mathrm{~Hz}), 1.49-1.43(\mathrm{~m}, 2 \mathrm{H}) ;{ }^{13} \mathrm{C}-\mathrm{NMR}(100$ $\left.\mathrm{MHz}, \mathrm{CDCl}_{3}\right) ; \delta=170.7,168.6,148.1,139.8,129.3,128.8,127.5,116.3,73.0,52.6(2 \mathrm{C}), 33.7,28.5,25.6,21.1 ; \mathrm{IR}($ thin film) $v_{\max }=3005,2953,2879,17.28,1625,1494,1437,1333,1293,1213,1043,864,753$, 692; HRMS calc. for $\mathrm{C}_{18} \mathrm{H}_{21} \mathrm{NO}_{5}=331.1418$, exp. $=331.1420$ 


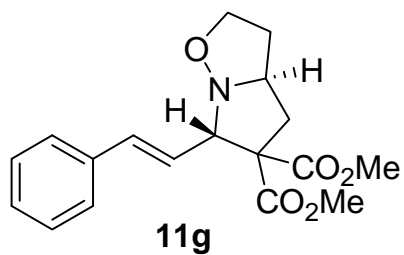

Compound 11g was prepared according to General Procedure B. The amounts of reagents employed were: oxime ether 9g-trans $(0.140 \mathrm{~g}, 0.42 \mathrm{mmol}), \mathrm{Yb}(\mathrm{OTf})_{3} \bullet \mathrm{xH}_{2} \mathrm{O}(0.013 \mathrm{~g}, 0.02 \mathrm{mmol})$. Compound $11 \mathrm{~g}(0.127 \mathrm{~g}, 0.38 \mathrm{mmol}, 90$ \% $)$ was obtained as a white solid. $\mathrm{mp}=84-86{ }^{\circ} \mathrm{C} ;{ }^{1} \mathrm{H}-\mathrm{NMR}\left(600 \mathrm{MHz}, \mathrm{CDCl}_{3}\right) ; \delta=7.38(\mathrm{~d}, 7.2 \mathrm{~Hz}, 2 \mathrm{H}), 7.28(\mathrm{dd}, J=7.2,7.2$ $\mathrm{Hz}, 2 \mathrm{H}), 7.21(\mathrm{dd}, J=7.2,7.2 \mathrm{~Hz}, 1 \mathrm{H}), 6.78(\mathrm{~d}, 16.2 \mathrm{~Hz}, 1 \mathrm{H}), 6.20(\mathrm{dd}, J=7.2,16.2 \mathrm{~Hz}, 1 \mathrm{H}), 4.53(\mathrm{~d}, J=7.2 \mathrm{~Hz}, 1 \mathrm{H})$, 4.11 (dddd, $J=7.8,7.8,7.8,8.4 \mathrm{~Hz}, 1 \mathrm{H}), 3.96\left(\mathrm{ddd}, J_{1-3}=7.8 \mathrm{~Hz}, 1 \mathrm{H}\right), 3.92(\mathrm{ddd}, J=4.2,8.4,8.4 \mathrm{~Hz}, 1 \mathrm{H}), 3.76(\mathrm{~s}, 3 \mathrm{H})$, $3.65(\mathrm{~s}, 3 \mathrm{H}), 2.90(\mathrm{dd}, J=7.8,13.8 \mathrm{~Hz}, 1 \mathrm{H}), 2.44(\mathrm{dddd}, J=7.8 .7 .8,7.8,12.0 \mathrm{~Hz}, 1 \mathrm{H}), 2.07(\mathrm{dd}, J=7.8,13.8 \mathrm{~Hz}, 1 \mathrm{H})$, 2.03 (dddd, $J=3.0,4.2,7.2,12.0 \mathrm{~Hz}, 1 \mathrm{H}) ;{ }^{13} \mathrm{C}-\mathrm{NMR}\left(100 \mathrm{MHz}, \mathrm{CDCl}_{3}\right) ; \delta=170.2,169.3,136.8,133.0,128.4,127.5$, 126.6, 125.1, 71.8, 64.8, 64.2,62.5, 52.9, 52.7, 38.5, 36.3; IR (thin film) $v_{\max }=3027,2954,2879,1735,1457,1449,1437$, 1277, 1210, 1178, 1125, 1104, 1031, 970, 916, 747, 695; HRMS calc. for $\mathrm{C}_{18} \mathrm{H}_{21} \mathrm{NO}_{5}=331.1418$, exp. $=331.1420$.

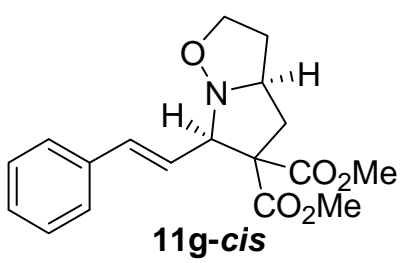

Compound 11g-cis was prepared according to General Procedure B. The amounts of reagents employed were: oxime ether 9g-cis $(0.090 \mathrm{~g}, 0.27 \mathrm{mmol})$ and $\mathrm{Yb}(\mathrm{OTf})_{3}{ }^{\bullet} \mathrm{xH}_{2} \mathrm{O}(0.08 \mathrm{~g}, 0.01 \mathrm{mmol})$. Compound 11g-cis (0.073 g, $0.22 \mathrm{mmol}$, $81 \%$ ) was obtained as a colourless oil. ${ }^{1} \mathrm{H}-\mathrm{NMR}\left(600 \mathrm{MHz}, \mathrm{CDCl}_{3}\right) ; \delta=7.2(\mathrm{dd}, 1.2,7.8 \mathrm{~Hz}, 2 \mathrm{H}), 7.30(\mathrm{dd}, J=7.8,7.8$ $\mathrm{Hz}, 2 \mathrm{H}), 7.22(\mathrm{ddd}, J=1.2,7.8,7.8 \mathrm{~Hz}, 1 \mathrm{H}), 6.71(\mathrm{~d}, J=12.6 \mathrm{~Hz}, 1 \mathrm{H}), 6.52(\mathrm{dd}, J=9.0,12.6 \mathrm{~Hz}, 1 \mathrm{H}), 4.52(\mathrm{~d}, J=7.8$ $\mathrm{Hz}, 1 \mathrm{H}), 3.94(\mathrm{ddd}, J=4.8,8.4,8.4 \mathrm{~Hz}, 1 \mathrm{H}), 3.94(\mathrm{~m}, 1 \mathrm{H}), 2.82(\mathrm{dd}, J=5.4,13.2 \mathrm{~Hz}, 1 \mathrm{H}), 3.78-3.70(\mathrm{~m}, 7 \mathrm{H}), 2.58$ (dddd, $J=4.2,8.4,8.4,12.4 \mathrm{~Hz}, 1 \mathrm{H}), 2.35(\mathrm{dd}, J=10.2,13.2 \mathrm{~Hz}, 1 \mathrm{H}), 2.29(\mathrm{dddd}, J=1.8,7.2,9.0,12.0 \mathrm{~Hz}, 1 \mathrm{H}){ }^{13} \mathrm{C}-$ $\operatorname{NMR}\left(100 \mathrm{MHz}, \mathrm{CDCl}_{3}\right) ; \delta=171.6,170.0,137.1,133.7,128.4,127.5,126.7,124.0,75.6,65.8,64.1,63.9,82.9,52.4$ 41.7, 36.5; IR (thin film) $v_{\max }=3027,2954,2879,1733,1495,1451,1435,1332,1260,1210,1176,1121,1096,1075$, $1059,1028,969,917,834,808,751,697$; HRMS calc. for $\mathrm{C}_{18} \mathrm{H}_{21} \mathrm{NO}_{5}=331.1421$, exp. $=331.1420$. 


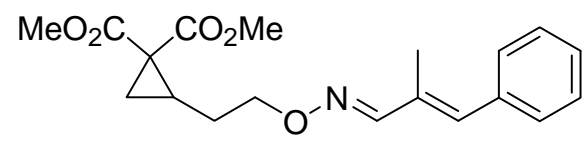

9h-trans

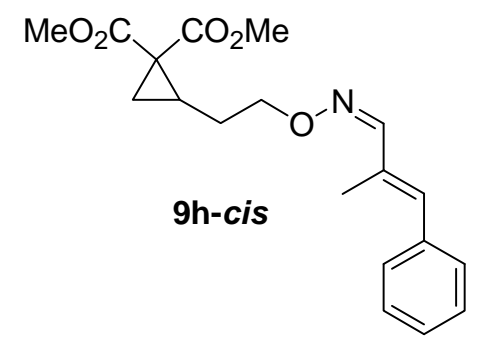

Compounds 9h-trans and 9h-cis were prepared according to General Procedure A. The amounts of reagents employed were: cyclopropane 17 (0.255 g, $1.17 \mathrm{mmol})$, $\alpha$-methyl-trans-cinnamaldehyde $(0.189 \mathrm{~g}, 1.29 \mathrm{mmol})$. A $2: 1$ mixture of trans to cis isomers was observed in the crude reaction mixture by ${ }^{1} \mathrm{H}$ NMR, which were separable to yield oxime ether 9h-trans $(0.171 \mathrm{~g}, 0.49 \mathrm{mmol}, 42 \%)$ and oxime ether $9 \mathrm{~h}$-cis $(0.010 \mathrm{~g}, 0.03 \mathrm{mmol}, 25 \%)$ both as colourless oils (67 \% overall yield).

9h-trans: ${ }^{1} \mathrm{H}-\mathrm{NMR}\left(600 \mathrm{MHz}, \mathrm{CDCl}_{3}\right) ; \delta=7.84(\mathrm{~s}, 1 \mathrm{H}), 7.37-7.35(\mathrm{~m}, 3 \mathrm{H}), 7.28-7.26(\mathrm{~m}, 1 \mathrm{H}), 6.62(\mathrm{~s}, 1 \mathrm{H}), 4.19(\mathrm{t}, J=$ $6.0 \mathrm{~Hz}, 1 \mathrm{H}$ ), 3.78 (s, 3H), 3.73 (s, 3H), 2.10 (s, 3H), 2.05 (dddd, $\left.J_{1-4}=7.8 \mathrm{~Hz}, 1 \mathrm{H}\right), 1.86$ (dddd, $J=6.6,6.6,6.6,14.4 \mathrm{~Hz}$, $1 \mathrm{H}), 1.61$ (dddd, $J=6.6,6.6,6.6,14.4 \mathrm{~Hz}, 1 \mathrm{H}), 1.48-1.42(\mathrm{~m}, 2 \mathrm{H}) ;{ }^{13} \mathrm{C}-\mathrm{NMR}\left(100 \mathrm{MHz}, \mathrm{CDCl}_{3}\right) ; \delta=170.7,168.6$, $153.8,136.5,136.0,132.2,129.3,128.3,127.5,72.7,52.6,52.5,33.7,28.5,25.6,21.0,13.1$; IR (thin film) $v_{\max }=3056$, 3025, 2954, 1729, 1438, 1333, 1291, 1267, 1214, 1131, 1076, 1062, 1052, 1032, 946, 906, 882, 753, 700; HRMS calc. for $\mathrm{C}_{19} \mathrm{H}_{23} \mathrm{NO}_{5}=345.1575$, exp. $=345.1576$.

9h-cis: ${ }^{1} \mathrm{H}-\mathrm{NMR}\left(600 \mathrm{MHz}, \mathrm{CDCl}_{3}\right) ; \delta=7.38-7.32(\mathrm{~m}, 4 \mathrm{H}), 7.29-7.26(\mathrm{~m}, 1 \mathrm{H}), 6.97(\mathrm{~s}, 1 \mathrm{H}), 6.88(\mathrm{~s}, 1 \mathrm{H}), 4.17(\mathrm{t}, J=6.0$ $\mathrm{Hz} 2 \mathrm{H}), 3.77$ (s, 3H), $3.72(\mathrm{~s}, 3 \mathrm{H}), 2.23(\mathrm{~s}, 3 \mathrm{H}), 2.04$ (dddd, $\left.J_{1-4}=8.4 \mathrm{~Hz}, 1 \mathrm{H}\right), 1.87$ (dddd, $\left.J=6.6,6.6,6.6,13.8 \mathrm{~Hz}, 1 \mathrm{H}\right)$, 1.60 (dddd, $J=6.6,6.6,6.6,13.8 \mathrm{~Hz}, 1 \mathrm{H}), 1.48-1.43(\mathrm{~m}, 2 \mathrm{H}) ;{ }^{13} \mathrm{C}-\mathrm{NMR}\left(100 \mathrm{MHz}, \mathrm{CDCl}_{3}\right) ; \delta=170.7,168.6,149.8$, $137.4,136.4,130.8,129.3,128.2,127.6,73.3,52.6,33.6,28.5,25.6,21.1,17.8$; IR (thin film) $v_{\max }=3005,2930,2850$, 1374, 1457, 1437, 1331, 1291, 1213, 1131, 1077, 1021, 907, 888, 754, 699; HRMS calc. for $\mathrm{C}_{19} \mathrm{H}_{23} \mathrm{NO}_{5}=345.1572$, exp. $=345.1576$. 


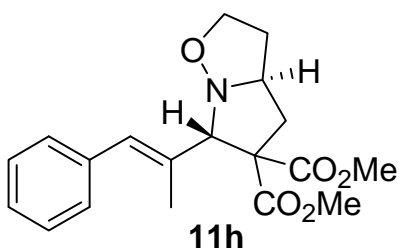

Compound 11h was prepared according to General Procedure B. The amounts of reagents employed were: oxime ether 9h-trans $(0.152 \mathrm{~g}, 0.44 \mathrm{mmol})$ and $\mathrm{Yb}(\mathrm{OTf})_{3} \bullet^{\times} \mathrm{H}_{2} \mathrm{O}(0.027 \mathrm{~g}, 0.04 \mathrm{mmol})$. Compound 11h (0.129 g, $0.37 \mathrm{mmol}, 85$ \%) was obtained as a colourless oil. ${ }^{1} \mathrm{H}-\mathrm{NMR}\left(600 \mathrm{MHz}, \mathrm{CDCl}_{3}\right) ; \delta=7.31-7.27(\mathrm{~m}, 4 \mathrm{H}), 7.20-2.17(\mathrm{~m}, 1 \mathrm{H}), 6.85(\mathrm{~s}, 1 \mathrm{H})$, $4.54(\mathrm{~s}, 1 \mathrm{H}), 4.11(\mathrm{dddd}, J=4.2,4.2,4.2,7.8 \mathrm{~Hz}, 1 \mathrm{H}), 4.02\left(\mathrm{ddd}, J_{1-3}=7.8 \mathrm{~Hz}, 1 \mathrm{H}\right), 3.94(\mathrm{ddd}, J=4.2,7.8 \mathrm{~Hz}, 3.78(\mathrm{~s}$, 3H), $3.63(\mathrm{~s}, 3 \mathrm{H}), 2.97$ (dd, $J=7.2,13.8 \mathrm{~Hz}, 1 \mathrm{H}), 2.44(\mathrm{dddd}, J=8.4,8.4,8.4,12.6 \mathrm{~Hz}, 1 \mathrm{H}), 2.04-1.99$ (m, $2 \mathrm{H}), 1.89$ (s, $3 \mathrm{H}) ;{ }^{13} \mathrm{C}-\mathrm{NMR}\left(100 \mathrm{MHz}, \mathrm{CDCl}_{3}\right) ; \delta=171.0,169.6,137.9,134.7,129.1,128.2,127.9,126.2,75.5,64.8,64.1,62.3$, 52.9, 52.6, 39.3, 36.3, 16.8; IR (thin film) $v_{\max }=3054,3023,2980,2881,1733,1491,1437,1325,1273,1255,1210$, $1179,1121,1080,1074,1055,1020,984,914,859,794,750,701$; HRMS calc. for $\mathrm{C}_{19} \mathrm{H}_{23} \mathrm{NO}_{5}=345.1579$, exp. $=$ 345.1576.

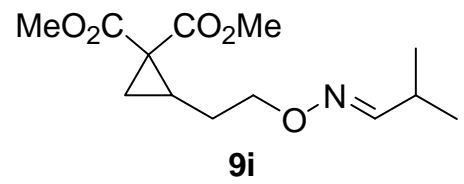

Compound 9i was prepared according to General Procedure A. The amounts of reagents employed were: cyclopropane $17(0.262 \mathrm{~g}, 1.21 \mathrm{mmol})$, isobutyraldehyde $(0.096 \mathrm{~g}, 1.33 \mathrm{mmol})$. An $8: 1$ mixture of trans to cis isomers was observed in the crude reaction mixture by ${ }^{1} \mathrm{H} \mathrm{NMR}$, which were practically inseparable and obtained as a colourless oil $(0.308 \mathrm{~g}$, $1.13 \mathrm{mmol}, 94 \%)$. A small analytical sample of the oxime ether 9i-trans was isolated by very careful column chromatography for characterization. 9i-trans: ${ }^{1} \mathrm{H}-\mathrm{NMR}\left(600 \mathrm{MHz}, \mathrm{CDCl}_{3}\right) ; \delta=7.26(\mathrm{~d}, J=6.6 \mathrm{~Hz}, 1 \mathrm{H}), 4.05(\mathrm{t}, J=6.6$ $\mathrm{Hz}, 2 \mathrm{H}), 3.75$ (s, 3H), 3.71 (s, 3H), 4.46 (d-septet, $J=6.6,6.6 \mathrm{~Hz}, 1 \mathrm{H}), 1.93$ (dddd, $\left.J_{1-4}=7.8 \mathrm{~Hz}, 1 \mathrm{H}\right), 1.79$ (dddd, $J=$ 6.6, 6.6, 6.6, $13.8 \mathrm{~Hz}, 1 \mathrm{H}), 1.52$ (dddd, $J=6.6,6.6,6.6,13.8 \mathrm{~Hz}, 1 \mathrm{H}), 1.44-1.39(\mathrm{~m}, 2 \mathrm{H}), 1.06(\mathrm{~d}, J=6.6 \mathrm{~Hz}, 6 \mathrm{H}) ;{ }^{13} \mathrm{C}-$ NMR $\left(100 \mathrm{MHz} \mathrm{CDCl}_{3}\right) ; \delta=170.7,168.5,155.8,71.9,52.5,52.5,33.6,29.3,28.4,25.5,21.0,20.0$; IR (thin film) $v_{\max }$ $=2962,2874,1735,1438,1368,1333,1291,1212,1131,1047,923,890,705 ;$ HRMS calc. for $\mathrm{C}_{13} \mathrm{H}_{21} \mathrm{NO}_{5}=271.1414$, exp. $=271.1420$. 


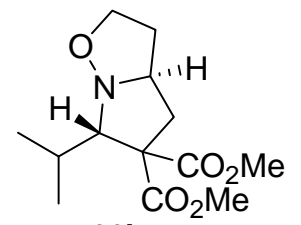

$11 i$

Compound 11i was prepared according to General Procedure B. The amounts of reagents employed were: a mixture of oxime ethers 9i-trans and 9i-cis $(8: 1,0.263 \mathrm{~g}, 0.97 \mathrm{mmol})$, and $\mathrm{Yb}(\mathrm{OTf})_{3} \bullet^{\bullet} \mathrm{H}_{2} \mathrm{O}(0.030 \mathrm{~g}, 0.05 \mathrm{mmol})$. A practically inseparable mixture of compound $\mathbf{1 1 i}$ and 211 was obtained as a colourless oil (8 : 1, $0.216 \mathrm{~g}, 0.79 \mathrm{mmol}, 82 \%$, vide infra for spectral data for 211). A small analytical sample of the 11i was isolated by very careful column chromatography for characterization. 11i: ${ }^{1} \mathrm{H}-\mathrm{NMR}\left(600 \mathrm{MHz}, \mathrm{CDCl}_{3}\right) ; \delta=4.03(\mathrm{q}, J=7.8 \mathrm{~Hz}, 1 \mathrm{H}), 3.87-3.81(\mathrm{~m}, 2 \mathrm{H}), 3.73(\mathrm{~s}, 3 \mathrm{H}, 3.73$ (s, 3H), $3.61(\mathrm{~d}, J=7.2 \mathrm{~Hz}, 1 \mathrm{H}), 2.75(\mathrm{dd}, J=7.2,13.2 \mathrm{~Hz}, 1 \mathrm{H}), 2.33$ (dddd, $J=8.4,8.4,8.4,12.0 \mathrm{~Hz}, 1 \mathrm{H}), 1.95-1.89$ (m, 3H), $0.99(\mathrm{dd}, J=3.6,7.2 \mathrm{~Hz}, 6 \mathrm{H}) ;{ }^{13} \mathrm{C}-\mathrm{NMR}\left(100 \mathrm{MHz}, \mathrm{CDCl}_{3}\right) ; \delta=171.2,170.2,76.6,63.7,62.9,52.8,52.5,40.6$, 36.2, 30.0, 21.8, 19.7; IR (thin film) $v_{\max }=2957,2875,1734,1437,1254,1214,1175,1094,986$; HRMS calc. for $\mathrm{C}_{13} \mathrm{H}_{21} \mathrm{NO}_{5}=271.1421$, exp. $=271.1420$.

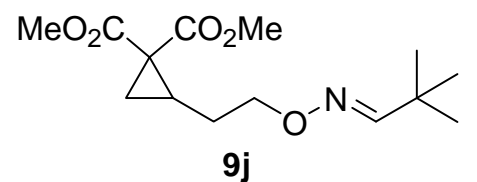

Compound 9j was prepared according to General Procedure A. The amounts of reagents employed were: cyclopropane 17 (0.750 g, $3.45 \mathrm{mmol})$, trimethylacetaldehyde (0.327 g, $3.80 \mathrm{mmol})$. Compound 9j (0.951 g, $3.33 \mathrm{mmol}, 96 \%)$ was isolated as a colourless oil as only the trans isomer. ${ }^{1} \mathrm{H}-\mathrm{NMR}\left(600 \mathrm{MHz}, \mathrm{CDCl}_{3}\right) ; \delta=7.27(\mathrm{~s}, 1 \mathrm{H}), 4.05(\mathrm{t}, J=6.6 \mathrm{~Hz}$, 2H), $3.75(\mathrm{~s}, 3 \mathrm{H}), 3.71(\mathrm{~s}, 3 \mathrm{H}), 1.99\left(\mathrm{dddd}, J_{1-4}=7.8 \mathrm{~Hz}, 1 \mathrm{H}\right), 1.79(\mathrm{dddd}, J=6.0,6.0,6.0,14.4 \mathrm{~Hz}, 1 \mathrm{H}), 1.51(\mathrm{dddd}, J=$ 6.6, 6.6, 6.6, $14.4 \mathrm{~Hz}, 1 \mathrm{H}), 1.44-1.38(\mathrm{~m}, 2 \mathrm{H}), 1.07(\mathrm{~s}, 9 \mathrm{H}) ;{ }^{13} \mathrm{C}-\mathrm{NMR}\left(100 \mathrm{MHz}, \mathrm{CDCl}_{3}\right) ; \delta=170.7,168.5,158.3,71.9$, $52.6,52.5,33.7,33.5,28.5,27.5,25.6,21.0 ;$ IR (thin film) $v_{\max }=2958,2873,1731,1458,1395,1367,1333,1292,1213$, 1132, 1065, 1035, 990, 944, 918, 886, 705; HRMS calc. for $\mathrm{C}_{14} \mathrm{H}_{23} \mathrm{NO}_{5}=285.1567$, exp. $=285.1576$. 


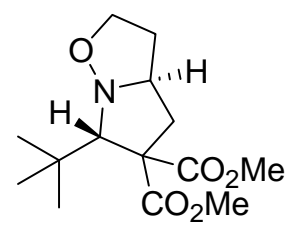

11j

Compound 11j was prepared according to General Procedure B. The amounts of reagents employed were: oxime ether $\mathbf{9 j}$

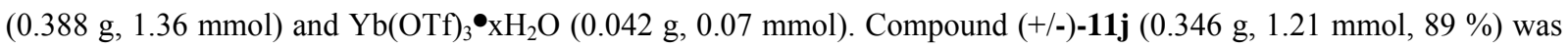
obtained as a white solid. $\left.(+/-)-\mathbf{1 1} \mathbf{j}, \mathrm{mp}=48-50{ }^{\circ} \mathrm{C} ;(2 \mathrm{~S}, 5 \mathrm{R})-\mathbf{1 1} \mathbf{j}, \mathrm{mp}=53-54{ }^{\circ} \mathrm{C} ; .{ }^{1} \mathrm{H}-\mathrm{NMR}(600 \mathrm{MHz}, \mathrm{CDCl})_{3}\right) ; \delta=$ $4.16(\mathrm{q}, J=7.8 \mathrm{~Hz}, 2 \mathrm{H}), 3.84(\mathrm{~s}, 1 \mathrm{H}), 3.82-3.79(\mathrm{~m}, 2 \mathrm{H}), 3.72(\mathrm{~s}, 3 \mathrm{H}), 3.70(\mathrm{~s}, 3 \mathrm{H}), 2.64(\mathrm{dd}, J=6.6,12.6 \mathrm{~Hz}, 1 \mathrm{H}), 2.35$ (dddd, $J=8.4,8.4,8.4,12.6 \mathrm{~Hz}, 1 \mathrm{H}), 1.94-1.90(\mathrm{~m}, 1 \mathrm{H}), 1.86(\mathrm{dd}, J=10.8,12.6 \mathrm{~Hz}, 1 \mathrm{H}), 0.99(\mathrm{~s}, 9 \mathrm{H}) ;{ }^{13} \mathrm{C}-\mathrm{NMR}(100$ $\left.\mathrm{MHz}, \mathrm{CDCl}_{3}\right) ; \delta=171.4,171.3,80.5,63.7,63.1,52.5,52.7,52.3,42.9,36.0,34.3,27.5 ;$ IR (thin film) $v_{\max }=2956,2875$, $1733,1483,1436,1397,1367,1274,1252,1216,1197,1178,1128,1112,1056,1020,986,955,926$, 820; HRMS calc. for $\mathrm{C}_{14} \mathrm{H}_{23} \mathrm{NO}_{5}=285.1570$, exp. $=285.1576 ;(2 S, 5 R)-\mathbf{1 1} \mathbf{j},[\alpha]_{\mathrm{D}}^{22}=-17^{\circ}\left(\mathrm{c} 0.3, \mathrm{CH}_{2} \mathrm{Cl}_{2}\right) . \%$ ee $=>99 \%$, based on $(+)-$ $\mathrm{Eu}(\mathrm{hcf})_{3}{ }^{1} \mathrm{H}-\mathrm{NMR}$ analysis of the well separated methyl ester signals (vide infra for spectra).

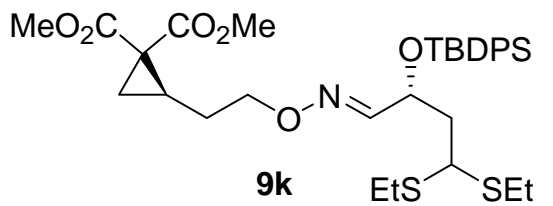

Compound 9k was prepared according to General Procedure A. The amounts of reagents employed were: cyclopropylalkoxylamine (S)-17 (0.875 g, $4.03 \mathrm{mmol}),(R)$-2-silyloxyaldehyde $\mathbf{1 8}^{5}$ (1.84 g, $\left.4.12 \mathrm{mmol}\right), 85 \mathrm{~mL}$ of total solvent, and $\mathrm{MgSO}_{4}(6.0 \mathrm{~g})$. After $21 \mathrm{~h}$, the reaction was filtered and concentrated to give a $20: 1$ mixture of trans to cis isomers by ${ }^{1} \mathrm{H}$ NMR, which was used directly in the next step without purification (2.56 g, $98 \%$ yield). A small sample of the trans-oxime ether was purified by careful column chromatography for characterization. 9k; ${ }^{1} \mathrm{H}-\mathrm{NMR}(600 \mathrm{MHz}$, $\left.\mathrm{CDCl}_{3}\right) ; \delta=7.68(\mathrm{dd}, J=8.4,1.2 \mathrm{~Hz}, 2 \mathrm{H}), 7.63(\mathrm{dd}, J=8.4,1.2 \mathrm{~Hz}, 2 \mathrm{H}), 7.44-7.35(\mathrm{~m}, 6 \mathrm{H}), 7.23(\mathrm{~d}, J=7.2 \mathrm{~Hz}, 1 \mathrm{H})$, $4.65\left(\mathrm{ddd}, J_{1-3}=6.6 \mathrm{~Hz}, 1 \mathrm{H}\right), 3.94-3.88(\mathrm{~m}, 3 \mathrm{H}), 3.73(\mathrm{~s}, 3 \mathrm{H}), 3.72(\mathrm{~s}, 3 \mathrm{H}), 2.60-2.45(\mathrm{~m}, 3 \mathrm{H}), 2.38(\mathrm{ddd}, J=7.2,7.2$, 7.2, $6.0 \mathrm{~Hz}$ ), 2.13 (quint, $J=7.2 \mathrm{~Hz}, 1 \mathrm{H}), 2.01(\mathrm{ddd}, J=12.4,7.8,6.6 \mathrm{~Hz}, 1 \mathrm{H}), 1.93-1.88(\mathrm{~m}, 1 \mathrm{H}), 1.65(\mathrm{dddd}, J=13.8$, 6.6, 6.6, $6.6 \mathrm{~Hz}, 1 \mathrm{H}), 1.44(\mathrm{dddd}, J=14.4,7.2,7.2,7.2 \mathrm{~Hz}, 1 \mathrm{H}), 1.41(\mathrm{dd}, J=9.6,4.8 \mathrm{~Hz}, 1 \mathrm{H}), 1.35(\mathrm{dd}, J=7.8,4.8 \mathrm{~Hz}$,

${ }^{5}$ For preparation of aldehyde 18, see: a) Khanapure, S. P.; Kim, S.; Penrose, J. F.; Austen, F. K; Powell, W. S.; Rokach, J. Tetrahedron Lett. 2002, 43, 6063-6066; b) Just, G.; Wang, Z. Y. J. Org. Chem. 1986, 51, 4796-4802. 
$1 \mathrm{H}), 1.17(t, \mathrm{~J}=7.8 \mathrm{~Hz}, 3 \mathrm{H}), 1.16(t, \mathrm{~J}=7.8 \mathrm{~Hz}, 3 \mathrm{H}), 1.06(\mathrm{~s}, 9 \mathrm{H}) ;{ }^{13} \mathrm{C}-\mathrm{NMR}\left(100 \mathrm{MHz}, \mathrm{CDCl}_{3}\right) ; \delta=170.52,168.38$, $150.98,135.86,135.81,133.30,133.17,129.73,129.65,127.57,127.47,72.24,69.00,52.51,52.43,46.53,42.29,33.56$, $28.28,26.88,25.16,23.71,23.65,20.83,19.22,14.26,14.21$; IR (thin film) $v_{\max }=2931,2859,1743,1437,1332,1291$, $1265,1212,1112,740,703$; HRMS calc. For $\mathrm{C}_{33} \mathrm{H}_{47} \mathrm{NO}_{6} \mathrm{~S}_{2} \mathrm{Si}=645.2642$, exp. $=645.2614$.

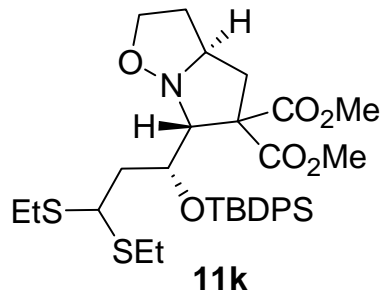

Compound 11k was prepared according to General Procedure B. The amounts of reagents employed were: crude oxime ether 9k (2.56 g, $3.96 \mathrm{mmol})$ and $\mathrm{Yb}(\mathrm{OTf})_{3} \bullet \mathrm{xH}_{2} \mathrm{O}(0.125 \mathrm{~g}, 0.20 \mathrm{mmol})$ in $\mathrm{CH}_{2} \mathrm{Cl}_{2}(30 \mathrm{ml})$ at $45{ }^{\circ} \mathrm{C}$ overnight. Compound 11k (2.51 g, $0.68 \mathrm{mmol}, 96 \%$ yield, 2 steps) was obtained as an oil (20:1, trans : cis, inseparable) after purification by column chromatography. ${ }^{1} \mathrm{H}-\mathrm{NMR}\left(600 \mathrm{MHz}, \mathrm{CDCl}_{3}\right) ; \delta=7.74(\mathrm{t}, J=7.8 \mathrm{~Hz}, 4 \mathrm{H}), 7.42-7.37(\mathrm{~m}, 6 \mathrm{H})$, $4.60(\mathrm{dt}, J=8.4,4.2, \mathrm{~Hz}, 1 \mathrm{H}), 4.50(\mathrm{~d}, J=8.4 \mathrm{~Hz}, 1 \mathrm{H}), 3.94-3.90(\mathrm{~m}, 1 \mathrm{H}), 3.74-3.70(\mathrm{~m}, 2 \mathrm{H}), 3.72(\mathrm{~s}, 3 \mathrm{H}), 3.60(\mathrm{~s}$, 3H), 3.47 (ddd, $\left.J_{1-4}=7.8 \mathrm{~Hz}, 1 \mathrm{H}\right), 2.94(\mathrm{dd}, J=13.8,9.0 \mathrm{~Hz}, 1 \mathrm{H}), 2.49$ (dd, $\left.J=13.8,6.0 \mathrm{~Hz}, 1 \mathrm{H}\right), 2.46$ (dddd, $J=12.0$, 8.4, 7.8, 4.2 Hz, 1H), $2.39-2.31(\mathrm{~m}, 4 \mathrm{H}), 2.16-2.11(\mathrm{~m}, 1 \mathrm{H}), 1.93$ (dddd, $J=12.0,7.8,7.8,7.8, \mathrm{~Hz}, 1 \mathrm{H}), 1.72(\mathrm{ddd}, J$ $=14.4,10.2,4.2 \mathrm{~Hz}, 1 \mathrm{H}), 1.09(\mathrm{t}, J=7.8 \mathrm{~Hz}, 3 \mathrm{H}), 1.05(\mathrm{t}, J=7.8 \mathrm{~Hz}, 3 \mathrm{H}), 0.97(\mathrm{~s}, 9 \mathrm{H}) ;{ }^{13} \mathrm{C}-\mathrm{NMR}\left(100 \mathrm{MHz}, \mathrm{CDCl}_{3}\right) ; \delta$ $=171.28,169.30,135.85,135.83,134.40,132.94,129.55,129.32,127.49,127.31,74.37,71.21,64.48,64.46,64.26$, $53.02,52.52,47.52,40.51,40.03,37.03,26.82,23.85,22.82,19.31,14.24$; IR (thin film) $v_{\max }=2957,2930,2858,1735$, 1429, 1263, 1106, 740,704; HRMS calc. for $\mathrm{C}_{33} \mathrm{H}_{47} \mathrm{NO}_{6} \mathrm{~S}_{2} \mathrm{Si}=645.2635$, exp. $=645.2614 .\left(2 R, 5 R, 1^{\prime} R\right),[\alpha]_{\mathrm{D}}^{22}=-$ $29.9^{\circ}\left(\mathrm{c} 7.4, \mathrm{CH}_{2} \mathrm{Cl}_{2}\right)$

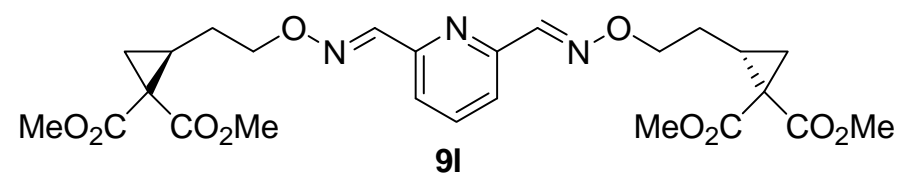

Compound 9l was prepared according to General Procedure A. The amounts of reagents employed were: cyclopropylalkoxylamine $(S)-17$ (0.485 g, $2.23 \mathrm{mmol})$, pyridine-2,6-dicarboxaldehyde (0.173 g, $1.00 \mathrm{mmol})$. Compound 91 (0.455 g, 
$0.85 \mathrm{mmol}, 85 \%)$ was isolated as a colourless oil as only the $E / E$ isomer. $\left.{ }^{1} \mathrm{H}-\mathrm{NMR}(600 \mathrm{MHz}, \mathrm{CDCl})_{3}\right) ; \delta=8.16(\mathrm{~s}, 2 \mathrm{H})$, $7.77(\mathrm{~d}, 2 \mathrm{H}, J=8.4 \mathrm{~Hz}), 7.69(\mathrm{t}, 1 \mathrm{H}, J=7.8 \mathrm{~Hz}), 4.29(\mathrm{t}, 4 \mathrm{H}, J=6.6 \mathrm{~Hz}), 3.77(\mathrm{~s}, 6 \mathrm{H}), 3.72(\mathrm{~s}, 6 \mathrm{H}), 2.07-2.02(\mathrm{~m}, 2 \mathrm{H})$, 1.89 (dddd, $2 \mathrm{H}, J=14.4,6.6,6.6,6.6 \mathrm{~Hz}), 1.65$ (dddd, $2 \mathrm{H}, J=14.4,7.2,7.2,7.2 \mathrm{~Hz}), 1.48-1.43(\mathrm{~m}, 4 \mathrm{H}) ;{ }^{13} \mathrm{C}-\mathrm{NMR}$ $\left(100 \mathrm{MHz}, \mathrm{CDCl}_{3}\right) ; \delta=170.6,168.6,151.7,149.2,136.8,120.9,73.5,52.6(2 \mathrm{C}), 33.7,28.5,25.4,21.0$; IR (thin film) $v_{\max }=3003,2953,2898,2850,1734,1458,1438,1334,1291,1214,1198,1132,1074,1044,991,912$, 739; HRMS calc. for $\mathrm{C}_{25} \mathrm{H}_{31} \mathrm{~N}_{3} \mathrm{O}_{10}=533.1997$, exp. $=533.2010$.

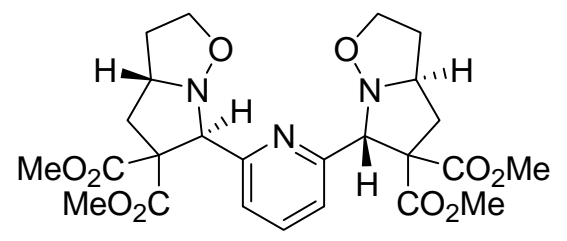

11)

Compound 111 was prepared according to General Procedure B. The amounts of reagents employed were: oxime ether 9l $(0.445 \mathrm{~g}, 0.83 \mathrm{mmol})$ and $\mathrm{Yb}(\mathrm{OTf})_{3} \bullet^{\times} \mathrm{H}_{2} \mathrm{O}(0.05 \mathrm{~g}, 0.08 \mathrm{mmol})$. Compound $11 \mathrm{l}(0.311 \mathrm{~g}, 0.58 \mathrm{mmol}, 70 \%)$ was obtained as a white solid after precipitation out of diethyl ether. No further purification was required. $\mathrm{mp}=170-173{ }^{\circ} \mathrm{C}$; ${ }^{1} \mathrm{H}-\mathrm{NMR}\left(600 \mathrm{MHz}, \mathrm{CDCl}_{3}\right) ; \delta=7.63(\mathrm{t}, 1 \mathrm{H}, J=7.8 \mathrm{~Hz}), 7.35(\mathrm{~d}, 2 \mathrm{H}, J-8.4 \mathrm{~Hz}), 5.40$ (s, 2H), 4.19 (broad q, 2H, $J=$ $7.2 \mathrm{~Hz}), 3.92(\mathrm{q}, 2 \mathrm{H}, J=7.8 \mathrm{~Hz}), 3.78(\mathrm{~s}, 6 \mathrm{H}), 3.78-3.75(\mathrm{~m}, 2 \mathrm{H}), 3.29(\mathrm{~s}, 6 \mathrm{H}), 3.10(\mathrm{dd}, 2 \mathrm{H}, J=13.2,9.0 \mathrm{~Hz}), 2.52-$ $2.46(\mathrm{~m}, 2 \mathrm{H}), 2.42(\mathrm{dd}, 2 \mathrm{H}, J=13.8,6.6 \mathrm{~Hz}), 2.21-2.17(\mathrm{~m}, 2 \mathrm{H}) ;{ }^{13} \mathrm{C}-\mathrm{NMR}\left(100 \mathrm{MHz}, \mathrm{CDCl}_{3}\right) ; \delta=170.5,169.1,156.8$, $136.7,122.7,76.6,65.6,65.0,63.5,53.1,52.3,39.2,36.4$; IR (thin film) $v_{\max }=2955,2906,2881,1734,1593,1577,1456$, $1435,1348,1326,1260,1219,1175,1122,1083$, 999, 916, 731; HRMS calc. for $\mathrm{C}_{25} \mathrm{H}_{31} \mathrm{~N}_{3} \mathrm{O}_{10}=533.2008$, exp. $=$ 533.2010; $\left(2 R, 5 S, 2^{\prime} R, 5^{\prime} S\right)-111,[\alpha]_{\mathrm{D}}^{22}=+95.7^{\circ}\left(\mathrm{c} 11.5, \mathrm{CH}_{2} \mathrm{Cl}_{2}\right)$.

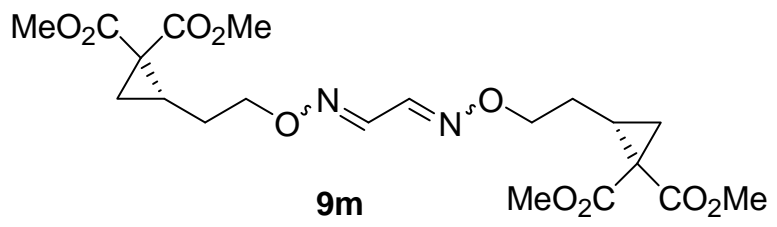

Compound 9m was prepared according to General Procedure A. The amounts of reagents employed were: cyclopropylalkoxylamine $(S)-17(0.459 \mathrm{~g}, 2.11 \mathrm{mmol})$, glyoxal $(0.061 \mathrm{~g}, 1.05 \mathrm{mmol})$. A $10: 8: 1 \mathrm{mixture}$ of $E / E: E / Z: Z / Z$ oxime ethers were obtained as a colourless oil $(0.379 \mathrm{~g}, 0.83 \mathrm{mmol}, 78 \%)$. The major $E / E$ isomer is denoted by * and the $Z / Z$ is denoted by \# in the ${ }^{1} \mathrm{H}$ NMR. ${ }^{1} \mathrm{H}-\mathrm{NMR}\left(600 \mathrm{MHz}, \mathrm{CDCl}_{3}\right) ; \delta=8.19(\mathrm{~d}, 1 \mathrm{H}, 9.6 \mathrm{~Hz}), 7.69$ (s, $\left.2 \mathrm{H}^{*}\right), 7.61$ (s, $2 \mathrm{H \# )} 7.09$ (d, $1 \mathrm{H}, J=9.0 \mathrm{~Hz}), 4.20-4.16\left(\mathrm{~m}, 4 \mathrm{H}, 4 \mathrm{H}^{*}, 4 \mathrm{H \#}\right), 3.74\left(\mathrm{~s}, 6 \mathrm{H}, 6 \mathrm{H}^{*}, 6 \mathrm{H} \#\right), 3.70\left(\mathrm{~s}, 6 \mathrm{H}, 6 \mathrm{H}^{*}, 6 \mathrm{H \#}\right), 1.99-1.93(\mathrm{~m}, 2 \mathrm{H}$, 
$\left.2 \mathrm{H}^{*}, 2 \mathrm{H} \#\right), 1.83-1.78\left(\mathrm{~m}, 2 \mathrm{H}, 2 \mathrm{H}^{*}, 2 \mathrm{H \#}\right), 1.61-1.55\left(\mathrm{~m}, 2 \mathrm{H}, 2 \mathrm{H}^{*}, 2 \mathrm{H} \#\right), 1.44-1.41\left(\mathrm{~m}, 2 \mathrm{H}, 2 \mathrm{H}^{*}, 2 \mathrm{H \#}\right), 1.40-1.37$ $\left(\mathrm{m}, 2 \mathrm{H}, 2 \mathrm{H}^{*}, 2 \mathrm{H} \#\right) ;{ }^{13} \mathrm{C}-\mathrm{NMR}\left(100 \mathrm{MHz}, \mathrm{CDCl}_{3}\right) ; \delta=170.5,168.4,145.0,141.9,139.8,73.7,73.5,52.6,52.5,33.6$, 28.3, 25.2, 25.1 (2C), 20.8; HRMS calc. for $\mathrm{C}_{20} \mathrm{H}_{28} \mathrm{~N}_{2} \mathrm{O}_{10}=456.1754$, exp. $=456.1744$.
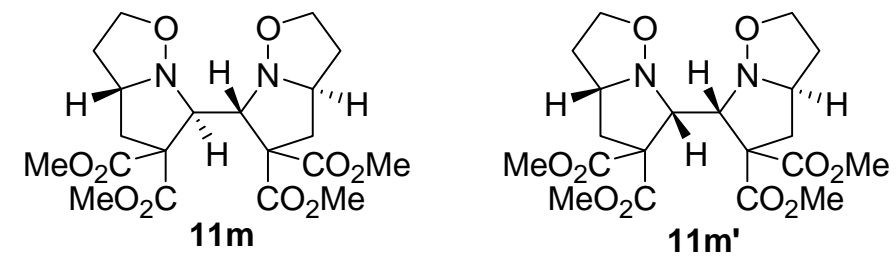

Compounds 11m and 11m' were prepared according to General Procedure B. The amounts of reagents employed were: a

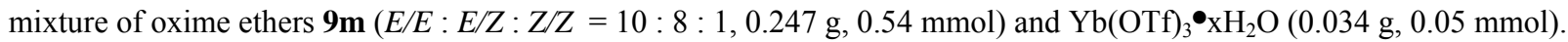
Compound 11m (0.119 g, 0.26 mmol, $48 \%$ ), was obtained as a white solid while compound 11m’ (0.087 g, 0.19 mmol, $35 \%$ ) was obtained as a yellow liquid (73\% overall yield).

11m: $\mathrm{mp}=116-11{ }^{\circ} \mathrm{C} ;{ }^{1} \mathrm{H}-\mathrm{NMR}\left(600 \mathrm{MHz}, \mathrm{CDCl}_{3}\right) ; \delta=4.77(\mathrm{~s}, 2 \mathrm{H}), 3.98(\mathrm{dddd}, 2 \mathrm{H}, J=6.0,6.0,6.0,1.8 \mathrm{~Hz}), 3.74$ $(\mathrm{s}, 6 \mathrm{H}), 3.73-3.70(\mathrm{~m}, 2 \mathrm{H}), 3.69(\mathrm{~s}, 6 \mathrm{H}), 3.56-3.53(\mathrm{~m}, 2 \mathrm{H}), 2.84(\mathrm{dd}, 2 \mathrm{H}, J=12.2,7.8 \mathrm{~Hz}), 2.35-2.30(\mathrm{~m}, 2 \mathrm{H}), 2.12$ $(\mathrm{dd}, 2 \mathrm{H}, J=12.2,7.2 \mathrm{~Hz}), 2.04-1.99(\mathrm{~m}, 2 \mathrm{H}) ;{ }^{13} \mathrm{C}-\mathrm{NMR}\left(100 \mathrm{MHz}, \mathrm{CDCl}_{3}\right) ; \delta=171.0,169.7,70.2,64.2,64.0,63.9$, 53.0, 52.5, 39.4, 36.5; IR (thin film) $v_{\max }=2855,2871,1733,1435,1258,1211,1178,1133,1100,1070,1030,918,735$; HRMS calc. for $\mathrm{C}_{20} \mathrm{H}_{128} \mathrm{~N}_{2} \mathrm{O}_{10}=456.1745$, exp. $=456.174 ;\left(2 R, 5 R, 2^{\prime} R, 5^{\prime} R\right)-11 \mathrm{~m},[\alpha]_{\mathrm{D}}^{22}=+22.9^{\circ}\left(\mathrm{c}^{2} .5, \mathrm{CH}_{2} \mathrm{Cl}_{2}\right)$.

11m': ${ }^{1} \mathrm{H}-\mathrm{NMR}\left(600 \mathrm{MHz}, \mathrm{CDCl}_{3}\right) ; \delta=5.45(\mathrm{~d}, 1 \mathrm{H}, J=12.0 \mathrm{~Hz}), 3.84-3.80(\mathrm{~m}, 3 \mathrm{H}), 3.78(\mathrm{~s}, 3 \mathrm{H}), 3.76(\mathrm{~s}, 3 \mathrm{H}), 3.76-$ $3.73(\mathrm{~m}, 1 \mathrm{H}), 3.65(\mathrm{~s}, 3 \mathrm{H}), 3.62(\mathrm{~s}, 3 \mathrm{H}), 3.62-3.58(\mathrm{~m}, 1 \mathrm{H}), 3.55(\mathrm{~d}, 1 \mathrm{H}, J=12.6 \mathrm{~Hz}), 3.50(\mathrm{q}, 1 \mathrm{H}, J=8.4 \mathrm{~Hz}), 3.00$ $(\mathrm{dd}, 1 \mathrm{H}, J=14.4,9.6 \mathrm{~Hz}), 2.72(\mathrm{dd}, 1 \mathrm{H}, J=13.2,3.6 \mathrm{~Hz}), 2.43(\mathrm{dd}, 1 \mathrm{H}, J=13.8,6.0 \mathrm{~Hz}), 2.43-2.39(\mathrm{~m}, 1 \mathrm{H}), 2.35-$ $2.30(\mathrm{~m}, 1 \mathrm{H}), 2.28(\mathrm{dd}, 1 \mathrm{H}, J=13.2,10.8 \mathrm{~Hz}), 2.18-2.13(\mathrm{~m}, 1 \mathrm{H}), 2.04-2.00(\mathrm{~m}, 1 \mathrm{H}) ;{ }^{13} \mathrm{C}-\mathrm{NMR}\left(100 \mathrm{MHz}^{\mathrm{CDCl}}\right)_{3}$; $\delta=172.5,171.8,170.4,170.2,52.9,71.0,66.1,65.2,63.5,52.5,62.0,52.9,52.7,52.3,43.4,40.6,36.9,36.4$; IR (thin film) $v_{\max }=2953,2878,1733,1455,1434,1338,1266,1216,1176,1105,1081,1028,918,840,730$; HRMS calc. for $\mathrm{C}_{20} \mathrm{H}_{28} \mathrm{~N}_{2} \mathrm{O}_{10}=456.1745$, exp. $=456.1744 ;\left(2 R, 5 R, 2^{\prime} S, 5^{\prime} R\right)-11 \mathbf{m}^{\prime},[\alpha]_{\mathrm{D}}^{22}=+93.1^{\circ}\left(\mathrm{c} 6.4, \mathrm{CH}_{2} \mathrm{Cl}_{2}\right)$. 


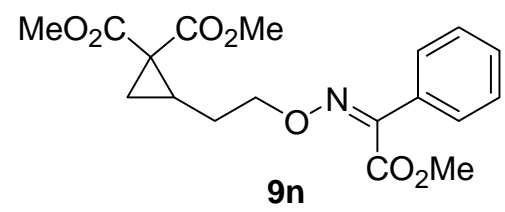

Compound 9n was prepared according to General Procedure A. The amounts of reagents employed were: cyclopropane 17 (0.250 g, $1.15 \mathrm{mmol})$, methyl benzoylformate (0.208 g, $1.27 \mathrm{mmol})$. Compound 9n (0.132 g, $0.36 \mathrm{mmol}, 32 \%)$ was obtained as a colourless oil as only the $Z$ isomer. ${ }^{6}{ }^{1} \mathrm{H}-\mathrm{NMR}\left(600 \mathrm{MHz}, \mathrm{CDCl}_{3}\right) ; \delta=7.55-7.54(\mathrm{~m}, 2 \mathrm{H}), 7.40-7.30(\mathrm{~m}$, 3H), 2.29 (ddd, $J=2.4,6.0,6.0 \mathrm{~Hz}, 2 \mathrm{H}), 3.94(\mathrm{~s}, 3 \mathrm{H}), 3.76(\mathrm{~s}, 3 \mathrm{H}), 3.71(\mathrm{~s}, 3 \mathrm{H}), 2.02\left(\mathrm{dddd}, J_{1-4}=7.8 \mathrm{~Hz}, 1 \mathrm{H}\right), 1.88$ (dddd, $J=6.0,6.0,6.0,14.4 \mathrm{~Hz}, 1 \mathrm{H}), 1.63$ (dddd, $J=6.6,6.6,6.6,14.4 \mathrm{~Hz}, 1 \mathrm{H}), 1.47-1.41(\mathrm{~m}, 2 \mathrm{H}) ;{ }^{13} \mathrm{C}-\mathrm{NMR}(100$ $\left.\mathrm{MHz}, \mathrm{CDCl}_{3}\right) ; \delta=170.6,168.5,164.0,150.8,130.4,130.1,128.7,126.2,73.8,52.6,52.3,33.6,28.6,25.2,21.0 ;$ IR (thin film) $v_{\max }=3005,2955,2849,1742,1499,1437,1335,1292,1223,1132,1076,1033,1019,1001,958,913,885,772$, 743, 683, 657; HRMS calc. for $\mathrm{C}_{18} \mathrm{H}_{21} \mathrm{NO}_{7}=363.1310$, exp. $=363.1318$.

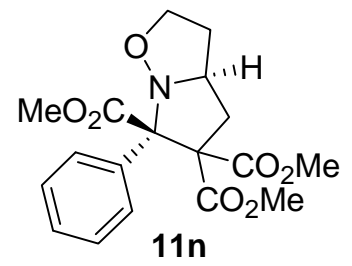

Compound 11n was prepared according to General Procedure B. The amounts of reagents employed were: oxime ether 9n (0.053 g, $0.15 \mathrm{mmol})$ and $\mathrm{Yb}(\mathrm{OTf})_{3} \bullet \mathrm{xH}_{2} \mathrm{O}(0.05 \mathrm{~g}, 0.08 \mathrm{mmol})$. Compound (+/-)-11n (0.053 g, $0.15 \mathrm{mmol}$, quantitative yield) was obtained as a yellow solid. $(+/-)-11 n \mathrm{mp}=112-116{ }^{\circ} \mathrm{C} ;(2 R, 5 R)-11 n$ was a yellow oil. ${ }^{1} \mathrm{H}-$ NMR $\left(600 \mathrm{MHz}, \mathrm{CDCl}_{3}\right) ; \delta=7.96-7.95(\mathrm{~m}, 2 \mathrm{H}), 7.03-7.23(\mathrm{~m}, 3 \mathrm{H}), 4.52\left(\mathrm{dddd}, J_{1-4}=7.2 \mathrm{~Hz}, 1 \mathrm{H}\right), 4.18-4.14(\mathrm{~m}, 1 \mathrm{H})$, $3.96\left(\mathrm{ddd}, J_{1-3}=7.8 \mathrm{~Hz}, 1 \mathrm{H}\right), 3.77(\mathrm{~s}, 3 \mathrm{H}), 3.69(\mathrm{~s}, 3 \mathrm{H}), 3.11(\mathrm{~s}, 3 \mathrm{H}), 2.94(\mathrm{dd}, J=9.6,12.6 \mathrm{~Hz}, 1 \mathrm{H}), 2.76(\mathrm{dd}, J=7.2$, $12.6 \mathrm{~Hz}, 1 \mathrm{H}), 2.46-2.40(\mathrm{~m}, 1 \mathrm{H}), 2.29(\mathrm{dddd}, J=7.2,7.2,7.2,12.0 \mathrm{~Hz}, 1 \mathrm{H}) ;{ }^{13} \mathrm{C}-\mathrm{NMR}\left(100 \mathrm{MHz}, \mathrm{CDCl}_{3}\right) ; \delta=171.6$, $169.9,169.5,137.8,128.8,127.6,127.1,81.8,72.3,69.5,63.0,52.7,52.6,52.3,39.6,36.1$; 1D-NOE correlation: H5(4.52 ppm) - ArH (7.96-7.95 ppm); IR (thin film) $v_{\max }=3000,2954,2927,1734,1496,1448,1435,1312,1248$, $1045,1005,988,914,870,860,803,760,732 ; \mathrm{HRMS}$ calc. for $\mathrm{C}_{18} \mathrm{H}_{21} \mathrm{NO}_{7}=363.1317$, exp. $=363.1318 ;(2 R, 5 R)-11 \mathbf{n}$, $[\alpha]_{\mathrm{D}}^{22}=+69.10^{\circ}\left(\mathrm{c} 1.1, \mathrm{CH}_{2} \mathrm{Cl}_{2}\right)$ HPLC (Chiracel OD-H, $7 \% \mathrm{iPrOH} /$ hexanes, $\left.1.25 \mathrm{~mL} / \mathrm{min}\right),(2 S, 5 S ; 2 R, 5 R) \mathrm{RT}=$ $6.66,12.04 \mathrm{~min},(2 R, 5 R) \mathrm{RT}=11.95 \mathrm{~min} ; \mathrm{ee}=>99 \%$.

\footnotetext{
${ }^{6} \mathrm{Z}$ geometry inferred from $1 \mathrm{D}$ NOE experiments on adduct $\mathbf{1 1 n}$.
} 


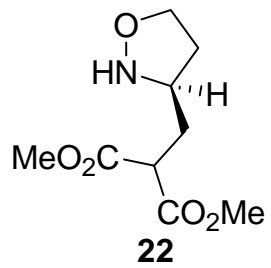

To a solution of alkoxylamine $17(0.22 \mathrm{~g}, 1.0 \mathrm{mmol})$ in $\mathrm{CH}_{2} \mathrm{Cl}_{2}(5 \mathrm{ml})$ was added $\mathrm{Yb}(\mathrm{OTf})_{3}(0.031 \mathrm{~g}$, $0.05 \mathrm{mmol})$. The solution was stirred at room temperature for $30 \mathrm{~min}$, diluted with $\mathrm{CH}_{2} \mathrm{Cl}_{2}(10 \mathrm{ml})$ and washed with $\mathrm{H}_{2} \mathrm{O}(3 \mathrm{x})$ and brine $(1 \mathrm{x})$. The organic layer was dried over $\mathrm{MgSO}_{4}$, filtered and concentrated in vacuo to afford $0.215 \mathrm{~g}(99 \%) 22$ as a colourless oil. ${ }^{1} \mathrm{H}-\mathrm{NMR}\left(400 \mathrm{MHz}, \mathrm{CDCl}_{3}\right)$; $\delta=5.52($ br s, $1 \mathrm{H}), 4.00(\mathrm{ddd}, J=8.8,8.8,4.8 \mathrm{~Hz}, 1 \mathrm{H}), 3.72-3.619(\mathrm{~m}, 2 \mathrm{H}) 3.71(\mathrm{~s}, 3 \mathrm{H}), 3.69(\mathrm{~s}$, 3H), 3.42 (dddd, $J=9.6,9.6,4.8,4.8 \mathrm{~Hz}, 1 \mathrm{H}), 2.34$ (dddd, $J=12.4,8.8,8.8,4.8 \mathrm{~Hz}, 1 \mathrm{H}) 2.07-1.95$ (m, 2H), 1.84 (dddd, $J=12.4,8.8,7.6,4.8 \mathrm{~Hz}, 1 \mathrm{H}) ;{ }^{13} \mathrm{C}-\mathrm{NMR}\left(100 \mathrm{MHz}, \mathrm{CDCl}_{3}\right) ; \delta=169.7,169.6$, 70.0, 57.8, 52.54, 52.50, 49.5, 35.7, 32.6; IR (thin film) $v_{\max }=3464,3231,2958,2883,1734,1437$, 1338, 1259, 1160, 1032; HRMS calc. for $\mathrm{C}_{9} \mathrm{H}_{15} \mathrm{NO}_{5}=217.0950$, exp. $=217.0951$.

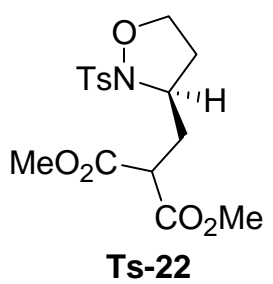

To a solution of alkoxylamine $17(0.16 \mathrm{~g}, 0.73 \mathrm{mmol})$ in $\mathrm{CH}_{2} \mathrm{Cl}_{2}(7 \mathrm{ml})$ was added $\mathrm{Yb}(\mathrm{OTf})_{3}(0.023 \mathrm{~g}, 0.037 \mathrm{mmol})$. The reaction mixture was stirred at room temperature for $30 \mathrm{~min}$ after which $\mathrm{TsCl}(0.15 \mathrm{~g}, 0.81 \mathrm{mmol}), \mathrm{NEt}_{3}(0.113 \mathrm{ml}$, $0.81 \mathrm{mmol})$ and DMAP $(\sim 0.002 \mathrm{~g}, 1 \mathrm{~mol} \%)$ were added. The mixture was stirred for an additional $1 \mathrm{hr}$ at room temperature and preabsorbed directly onto silica gel. Purification by FC (35\% EtOAc/hexanes) afforded 0.24 g (90 \%) Ts-22 as a colourless oil. ${ }^{1} \mathrm{H}-\mathrm{NMR}\left(600 \mathrm{MHz}^{\mathrm{CDCl}}{ }_{3}\right) ; \delta=7.81(\mathrm{~d}, J=8.4 \mathrm{~Hz}, 2 \mathrm{H}), 7.33(\mathrm{~d}, J=8.4 \mathrm{~Hz}, 2 \mathrm{H}), 4.40$ (dddd, $J=12.0,7.2,3.6,3.6 \mathrm{~Hz}, 1 \mathrm{H}), 4.07$ (ddd, $\left.J_{1-3}=7.2 \mathrm{~Hz}, 1 \mathrm{H}\right), 3.97\left(\mathrm{ddd}, J_{1-3}=7.8 \mathrm{~Hz}, 1 \mathrm{H}\right), 3.79(\mathrm{~s}, 3 \mathrm{H}), 3.75(\mathrm{dd}, J=$ $10.8,4.8 \mathrm{~Hz}, 1 \mathrm{H}), 3.70$ (s, 3H), 2.43 (s, 3H), 2.37 (dddd, $J=12.6,7.8,7.8,4.8 \mathrm{~Hz}, 1 \mathrm{H}), 2.23$ (ddd, J=12.0, 6.6, 3.6 Hz, 1H), 2.00-1.92 (m, 2H); ${ }^{13} \mathrm{C}-\mathrm{NMR}\left(150 \mathrm{MHz}, \mathrm{CDCl}_{3}\right) ; \delta=169.5,169.2,145.1,133.0,129.7,129.1,69.8,57.0,52.7$, 52.6, 48.9, 34.7, 34.0, 21.6; IR (thin film) $v_{\max }=2957,2849,1735,1598,1437,1333,1242,1166,1127,1092 ;$ HRMS 
calc. for $\mathrm{C}_{16} \mathrm{H}_{21} \mathrm{NO}_{7} \mathrm{~S}=371.1039$, exp. $=371.1028 ;(R)-\mathrm{Ts}-22,[\alpha]_{\mathrm{D}}^{22}=-3.9^{\circ}\left(\mathrm{c} 10.3, \mathrm{CH}_{2} \mathrm{Cl}_{2}\right)$; HPLC (Chiracel OD-H, $12 \% \mathrm{iPrOH} /$ hexanes, $1.2 \mathrm{ml} / \mathrm{min})(R / S)-\mathrm{Ts}-22, \mathrm{RT}=14.1,16.2 \mathrm{~min} ;(R)-\mathrm{Ts}-22, \mathrm{RT}=14.4 \mathrm{~min}$, ee $=>99 \%$.

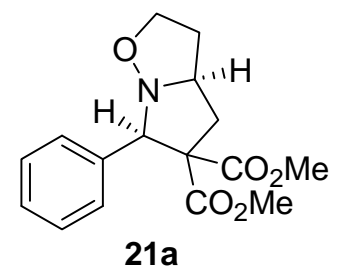

21a was prepared using General Procedure $C$ for cis-adducts. The amounts of reagents employed were: cyclopropylalkoxylamine 17 (0.22 g, $1.0 \mathrm{mmol})$, benzaldehyde (0.117 g, $1.10 \mathrm{mmol}), \mathrm{Yb}(\mathrm{OTf})_{3}(0.031 \mathrm{~g}, 0.05 \mathrm{mmol})$. Colourless oil, $0.30 \mathrm{~g}(98 \%) ;{ }^{1} \mathrm{H}-\mathrm{NMR}\left(600 \mathrm{MHz}, \mathrm{CDCl}_{3}\right) ; \delta=7.48(\mathrm{~d}, J-6.6 \mathrm{~Hz}, 2 \mathrm{H}), 7.23-7.17(\mathrm{~m}, 3 \mathrm{H}), 5.16(\mathrm{~s}, 1 \mathrm{H}), 4.03(\mathrm{ddd}, J=$ 8.4, 8.4, 6.0 Hz, 1H), 3.91 (add, $J=15.6,7.8 \mathrm{~Hz}, 1 \mathrm{H}), 3.82$ (ddd, $J=8.4,7.8,6.0 \mathrm{~Hz}, 1 \mathrm{H}), 3.69$ (s, $3 \mathrm{H}), 3.29(\mathrm{~s}, 3 \mathrm{H})$, $2.89(\mathrm{dd}, J=13.2,6.6 \mathrm{~Hz}, 1 \mathrm{H}), 2.47-2.38(\mathrm{~m}, 2 \mathrm{H}), 2.01(\mathrm{addd}, J=13.8,7.8,7.8 \mathrm{~Hz}, 1 \mathrm{H}) ;{ }^{13} \mathrm{C}-\mathrm{NMR}\left(100 \mathrm{MHz}, \mathrm{CDCl}_{3}\right)$; $\delta=172.1,169.8,135.4,129.5,127.6,127.5,76.1,66.3,64.8,64.6,53.0,51.9,41.6,34.8 ;$ IR $\left(\right.$ thin film) $v_{\max }=2953$, 2882, 1727, 1497, 1434, 1253, 1174, 4057, 1010; HRMS calc. for $\mathrm{C}_{16} \mathrm{H}_{19} \mathrm{NO}_{5}=305.1263$, exp. $=305.1256$.

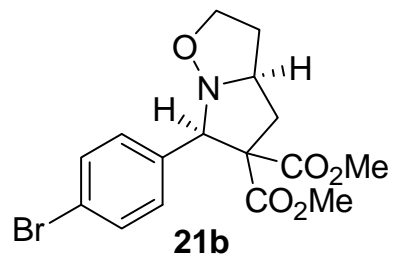

21b was prepared using General Procedure C for cis-adducts. The amounts of reagents employed were: cyclopropylalkoxylamine 17 (0.22 g, $1.0 \mathrm{mmol})$, 4-bromo-benzaldehyde (0.204 g, $1.10 \mathrm{mmol}), \mathrm{Yb}(\mathrm{OTf})_{3}(0.031 \mathrm{~g}, 0.05 \mathrm{mmol})$. Colourless oil, $0.34 \mathrm{~g}$ (88\%). Alternatively, compound 21b could be prepared from 9b-cis. The amounts of reagent employed were: oxime ether 9b-cis $(0.060 \mathrm{~g}, 0.02 \mathrm{mmol})$ and $\mathrm{Yb}(\mathrm{OTf})_{3} \bullet \mathrm{xH}_{2} \mathrm{O}(0.001 \mathrm{~g}, 0.002 \mathrm{mmol}) .0 .008 \mathrm{~g}(99 \%)$ of compound 21b was obtained as a single diastereomer. ${ }^{1} \mathrm{H}-\mathrm{NMR}\left(400 \mathrm{MHz}, \mathrm{CDCl}_{3}\right) ; \delta=7.46(\mathrm{~d}, J=8.4,2 \mathrm{H}), 7.40(\mathrm{~d}$, $J=8.4 \mathrm{~Hz}, 2 \mathrm{H}), 5.08(\mathrm{~s}, 1 \mathrm{H}), 4.05(\mathrm{ddd}, J=8.4,8.4,6.0 \mathrm{~Hz}, 1 \mathrm{H}), 3.93-3.84(\mathrm{~m}, 2 \mathrm{H}), 3.76(\mathrm{~s}, 3 \mathrm{H}), 3.39(\mathrm{~s}, 3 \mathrm{H}), 2.95(\mathrm{dd}$, $J=13.6,6.4 \mathrm{~Hz}, 1 \mathrm{H}), 2.57(\mathrm{dddd}, J=12.0,9.2,7.6,5.6 \mathrm{~Hz}, 1 \mathrm{H}), 2.42(\mathrm{dd}, J=13.6,9.6 \mathrm{~Hz}, 1 \mathrm{H}), 2.30-2.23(\mathrm{~m}, 1 \mathrm{H}) ;{ }^{13} \mathrm{C}-$ NMR (100 MHz, $\left.\mathrm{CDCl}_{3}\right) ; \delta=172.5,169.8,135.4,131.1,130.6,121.4,75.9,65.9,64.8,64.5,53.1,52.0,42.2,35.2 ; \mathrm{IR}$ (thin film) $v_{\max }=3459,2953,2882,2844,1734,1491,1436,1416,1254,1123,1061,1012$; HRMS calc. for $\mathrm{C}_{16} \mathrm{H}_{18} \mathrm{BrNO}_{5}=383.0368$, exp. $=383.0374$. 


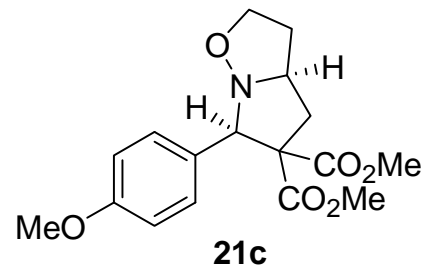

21c was prepared using General Procedure C for cis-adducts. The amounts of reagents employed were: cyclopropylalkoxylamine 17 (0.22 g, $1.0 \mathrm{mmol})$, 4-methoxy-benzaldehyde (0.150 g, $1.10 \mathrm{mmol}), \mathrm{Yb}(\mathrm{OTf})_{3}(0.031 \mathrm{~g}, 0.05 \mathrm{mmol})$. Colourless oil, $0.33 \mathrm{~g}(99 \%){ }^{1} \mathrm{H}-\mathrm{NMR}\left(400 \mathrm{MHz}, \mathrm{CDCl}_{3}\right) ; \delta=7.44(\mathrm{~d}, J=8.8 \mathrm{~Hz}, 2 \mathrm{H}), 6.77(\mathrm{~d}, J=8.8 \mathrm{~Hz}, 2 \mathrm{H}), 5.08(\mathrm{~s}$, 1H), 4.04 (ddd, $J=8.4,8.4,5.6 \mathrm{~Hz}, 1 \mathrm{H}), 3.86-3.80(\mathrm{~m}, 2 \mathrm{H}), 3.74(\mathrm{~s}, 3 \mathrm{H}), 3.70(\mathrm{~s}, 3 \mathrm{H}), 3.35(\mathrm{~s}, 3 \mathrm{H}), 2.91(\mathrm{dd}, J=13.6$, $7.2 \mathrm{~Hz}, 1 \mathrm{H}), 2.51$ (dddd, $J=12.4,9.2,7.2,5.2 \mathrm{~Hz}, 1 \mathrm{H}), 2.37$ (dd, $J=13.6,9.2 \mathrm{~Hz}, 1 \mathrm{H}), 2.22$ (dddd, $J=12.0,8.4,5.6$, $0.8 \mathrm{~Hz}, 1 \mathrm{H}) ;{ }^{13} \mathrm{C}-\mathrm{NMR}\left(100 \mathrm{MHz}, \mathrm{CDCl}_{3}\right) ; \delta=172.3,169.9,158.6,130.6,128.0,112.7,75.9,65.6,64.9,64.2,54.9$, 52.7, 51.8, 51.7, 34.8; IR (thin film) $v_{\max }=3034,2996,2990,2883,2839,1730,1613,1516,1457,1447,1309,1296$, 1208, 1058; HRMS calc. for $\mathrm{C}_{17} \mathrm{H}_{21} \mathrm{NO}_{6}=335.1369$, exp. $=335.1375$.

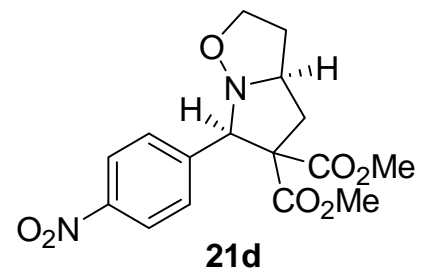

21d was prepared using General Procedure $\mathrm{C}$ for cis-adducts. The amounts of reagents employed were: cyclopropylalkoxylamine 17 (0.22 g, $1.0 \mathrm{mmol})$, 4-nitro-benzaldehyde (0.166 g, $1.10 \mathrm{mmol}), \mathrm{Yb}(\mathrm{OTf})_{3}(0.031 \mathrm{~g}, 0.05 \mathrm{mmol})$. Colourless oil, $0.32 \mathrm{~g}(91 \%) ;{ }^{1} \mathrm{H}-\mathrm{NMR}\left(400 \mathrm{MHz}, \mathrm{CDCl}_{3}\right) ; \delta=8.12(\mathrm{dd}, J=8.4,1.6 \mathrm{~Hz}, 2 \mathrm{H}), 7.77(\mathrm{dd}, J=8.4,1.6 \mathrm{~Hz}$, 2H), $5.17(\mathrm{~s}, 1 \mathrm{H}), 4.04(\mathrm{ddd}, J=8.4,8.4,6.0 \mathrm{~Hz}, 1 \mathrm{H}), 3.97-3.87(\mathrm{~m}, 2 \mathrm{H}), 3.79(\mathrm{~s}, 3 \mathrm{H}), 3.36(\mathrm{~s}, 3 \mathrm{H}), 2.97(\mathrm{dd}, J=13.6$, $6.0 \mathrm{~Hz}, 1 \mathrm{H}), 2.60(\mathrm{dddd}, J=12.0,9.6,7.6,5.6 \mathrm{~Hz}, 1 \mathrm{H}), 2.47$ (dd, $J=13.6,9.6 \mathrm{~Hz}, 1 \mathrm{H}), 2.29$ (dddd, $J=12.4,8.8,6.0$, $1.2,1 \mathrm{H}) ;{ }^{13} \mathrm{C}-\mathrm{NMR}\left(100 \mathrm{MHz}, \mathrm{CDCl}_{3}\right) ; \delta=172.3,169.3,146.9,144.2,129.9,122.5,75.6,66.0,64.7,64.4,53.3,52.0$, 42.4, 35.3; IR (thin film) $v_{\max }=3630,3117,2956,2885,1734,1602,1521,1437,1350,1257,1122,4066,1016$; HRMS calc. for $\mathrm{C}_{16} \mathrm{H}_{18} \mathrm{~N}_{2} \mathrm{O}_{7}=350.1114$, exp. $=350.1105$. 


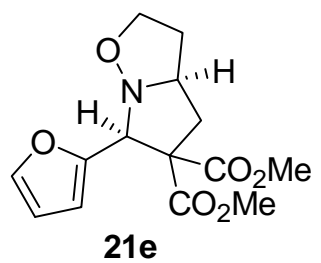

21e was prepared using General Procedure C for cis-adducts. The amounts of reagents employed were: cyclopropylalkoxylamine $17(0.22 \mathrm{~g}, 1.0 \mathrm{mmol})$, furan-2-carboxaldehyde (0.106 g, $1.10 \mathrm{mmol}), \mathrm{Yb}(\mathrm{OTf})_{3}(0.031 \mathrm{~g}, 0.05 \mathrm{mmol})$. Colourless oil, $0.25 \mathrm{~g}(85 \%) ;{ }^{1} \mathrm{H}-\mathrm{NMR}\left(400 \mathrm{MHz}, \mathrm{CDCl}_{3}\right) ; \delta=7.34(\mathrm{dd}, J=1.6,0.8 \mathrm{~Hz}, 1 \mathrm{H}), 6.43(\mathrm{ddd}, J=3.6,1.6$, $0.8 \mathrm{~Hz}, 1 \mathrm{H}), 6.30(\mathrm{dd}, J=3.6,1.6 \mathrm{~Hz}, 1 \mathrm{H}) 5.22(\mathrm{~s}, 1 \mathrm{H}), 3.95(\mathrm{ddd}, J=8.8,8.8,5.6 \mathrm{~Hz}, 1 \mathrm{H}), 3.89-3.79(\mathrm{~m}, 2 \mathrm{H}), 3.75(\mathrm{~s}$, 3H), 3.44 (s, 3H), 2.89 (dd, $J=13.6,6.8 \mathrm{~Hz}, 1 \mathrm{H}), 2.52(\mathrm{dddd}, J=14.4,8.8,7.2,5.2 \mathrm{~Hz}, 1 \mathrm{H}), 2.39(\mathrm{dd}, J=13.6,8.4 \mathrm{~Hz}$, 1H), 2.22 (dddd, $J=11.6,8.0,5.6,1.6 \mathrm{~Hz}, 1 \mathrm{H}) ;{ }^{13} \mathrm{C}-\mathrm{NMR}\left(150 \mathrm{MHz}, \mathrm{CDCl}_{3}\right) ; \delta=171.5,169.3,149.2,141.6,110.2$, 109.7, 70.1, 66.2, 64.2, 64.1, 53.1, 52.4, 40.8, 35.4; IR (thin film) $v_{\max }=2954,2883,1734,1437,1436,1256,1210,1175$, 1011; HRMS calc. for $\mathrm{C}_{14} \mathrm{H}_{17} \mathrm{NO}_{6}=295.1056$, exp. $=295.1064$.

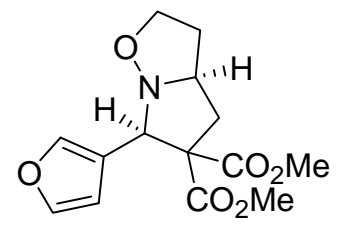

$21 f$

21f was prepared using General Procedure C for cis-adducts. The amounts of reagents employed were: cyclopropylalkoxylamine 17 (0.22 g, $1.0 \mathrm{mmol})$, furan-3-carboxaldehyde (0.106 g, $1.10 \mathrm{mmol}), \mathrm{Yb}(\mathrm{OTf})_{3}(0.031 \mathrm{~g}, 0.05 \mathrm{mmol})$. Colourless oil, $0.24 \mathrm{~g}(81 \%) ;{ }^{1} \mathrm{H}-\mathrm{NMR}\left(400 \mathrm{MHz}, \mathrm{CDCl}_{3}\right) ; \delta=7.49(\mathrm{dd}, J=1.2,0.8 \mathrm{~Hz}, 1 \mathrm{H}), 7.27(\mathrm{dd}, J=3.2,1.6 \mathrm{~Hz}$, 1H), $6.43(\mathrm{dd}, J=2.0,0.8 \mathrm{~Hz}, 1 \mathrm{H}), 4.98(\mathrm{~s}, 1 \mathrm{H}), 4.00(\mathrm{ddd}, J=8.4,8.4,5.2 \mathrm{~Hz}, 1 \mathrm{H}), 3.87-3.71(\mathrm{~m}, 2 \mathrm{H}), 3.69(\mathrm{~s}, 3 \mathrm{H})$, $3.42(\mathrm{~s}, 3 \mathrm{H}), 2.85(\mathrm{dd}, J=13.6,6.8 \mathrm{~Hz}, 1 \mathrm{H}), 2.52(\mathrm{dddd}, J=12.0,9.2,7.6,5.2 \mathrm{~Hz}, 1 \mathrm{H}), 2.29(\mathrm{dd}, J=13.6,9.2 \mathrm{~Hz}, 1 \mathrm{H})$, 2.28-2.21 (m, 1H); ${ }^{13} \mathrm{C}-\mathrm{NMR}\left(100 \mathrm{MHz}, \mathrm{CDCl}_{3}\right) ; \delta=171.7,169.7,141.7,141.5,119.7,111.7,69.0,65.6,64.4,64.1$, 52.7, 51.8, 41.2, 35.7; IR (thin film) $v_{\max }=3134,2954,2882,1729,1516,1436,1255,1211,1175,1122,1084,1062$, 1021; HRMS calc. for $\mathrm{C}_{14} \mathrm{H}_{17} \mathrm{NO}_{6}=295.1056$, exp. $=295.1050$. 


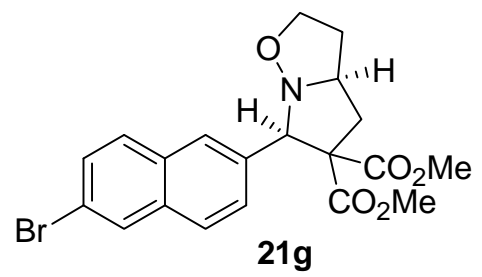

21g was prepared using General Procedure C for cis-adducts. The amounts of reagents employed were: cyclopropylalkoxylamine 17 (0.22 g, $1.0 \mathrm{mmol})$, 6-bromo-napthalene-2-carboxaldehyde (0.26 g, $1.10 \mathrm{mmol}), \mathrm{Yb}(\mathrm{OTf})_{3}(0.031 \mathrm{~g}$, $0.05 \mathrm{mmol})$. White solid, $0.43 \mathrm{~g}(100 \%) ;{ }^{1} \mathrm{H}-\mathrm{NMR}\left(600 \mathrm{MHz}, \mathrm{CDCl}_{3}\right) ; \delta=8.07(\mathrm{~s}, 1 \mathrm{H}), 7.95(\mathrm{~s}, 1 \mathrm{H}), 7.70(\mathrm{~d}, J=9.0$ $\mathrm{Hz}, 1 \mathrm{H}), 7.66(\mathrm{dd}, J=9.0 \mathrm{~Hz}, 1.8 \mathrm{~Hz}, 1 \mathrm{H}), 7.65(\mathrm{~d}, J=9.0 \mathrm{~Hz}, 1 \mathrm{H}), 7.50(\mathrm{dd}, J=9.0,1.8 \mathrm{~Hz}, 1 \mathrm{H}), 5.32(\mathrm{~s}, 1 \mathrm{H}), 4.12$ $\left(\mathrm{ddd}, J_{1-3}=8.4 \mathrm{~Hz}, 1 \mathrm{H}\right), 3.99-3.93(\mathrm{~m}, 2 \mathrm{H}), 3.77(\mathrm{~s}, 3 \mathrm{H}), 3.27(\mathrm{~s}, 3 \mathrm{H}), 3.01(\mathrm{dd}, J=13.8,6.6 \mathrm{~Hz}, 1 \mathrm{H}), 2.60(\mathrm{dddd}, J=$ $13.8,9.0,8.4,7.2 \mathrm{~Hz}, 1 \mathrm{H}), 2.48(\mathrm{dd}, J=13.8,9.0 \mathrm{~Hz}, 1 \mathrm{H}) 2.30(\mathrm{~m}, 1 \mathrm{H}) ;{ }^{13} \mathrm{C}-\mathrm{NMR}\left(100 \mathrm{MHz}, \mathrm{CDCl}_{3}\right) ; \delta=172.5,169.8$, $134.3,133.6,131.4,130.0,129.4,129.0,128.4,128.3,125.8,119.7,76.4,66.1,64.9,64.6,53.1,51.9,42.1,35.0 ;$ IR (thin film) $v_{\max }=3051,3027,2951,2880,2843,1719,1591,1500,1434,1332,1314,1252,1208,1174 \mathrm{~cm}^{-1}$; HRMS calc. for $\mathrm{C}_{20} \mathrm{H}_{20} \mathrm{BrNO}_{5}=433.0525$, exp. $=433.0521 ;(S)-21 \mathrm{~g},[\alpha]_{\mathrm{D}}^{22}=-10.2^{\circ}$ (c 2.9, $\left.\mathrm{CH}_{2} \mathrm{Cl}_{2}\right)$; HPLC (Chiracel OD-H, $1.75: 4: 94.25 \mathrm{EtOH} / \mathrm{MTBE} / \mathrm{hexanes}, 0.85 \mathrm{ml} / \mathrm{min}) \quad(2 S, 5 S ; 2 R, 5 R)-21 \mathrm{~g}, \mathrm{RT}=17.4,18.9 \mathrm{~min} ;(2 R, 5 R)-21 \mathrm{~g}, \mathrm{RT}=$ 19.0. $\min$, ee $=>99 \%$.

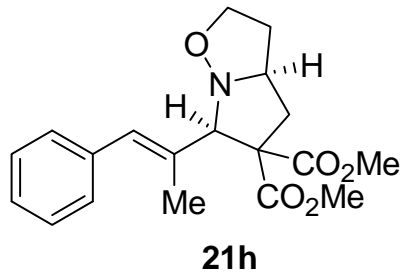

21h was prepared using General Procedure C for cis-adducts. The amounts of reagents employed were: cyclopropylalkoxylamine 17 (0.22 g, $1.0 \mathrm{mmol})$, trans-2-methyl-cinnamaldehyde ( $0.161 \mathrm{~g}, 1.10 \mathrm{mmol}), \mathrm{Yb}(\mathrm{OTf})_{3}(0.031 \mathrm{~g}, 0.05$ mmol). Colourless oil, $0.17 \mathrm{~g}(50 \%) .{ }^{1} \mathrm{H}-\mathrm{NMR}\left(600 \mathrm{MHz}, \mathrm{CDCl}_{3}\right) ; \delta=7.29-7.27(\mathrm{~m}, 2 \mathrm{H}), 7.23-7.24(\mathrm{~m}, 2 \mathrm{H}), 7.16(\mathrm{t}, J$ $=7.8 \mathrm{~Hz}, 1 \mathrm{H}), 6.92(\mathrm{~s}, 1 \mathrm{H}), 4.50(\mathrm{~s}, 1 \mathrm{H}), 3.92-3.81(\mathrm{~m}, 3 \mathrm{H}), 3.74(\mathrm{~s}, 3 \mathrm{H}), 3.68(\mathrm{~s}, 3 \mathrm{H}), 2.87(\mathrm{dd}, J=13.8,6.0 \mathrm{~Hz}, 1 \mathrm{H})$, 2.53 (dddd, $J=12.0,9.0,7.2,5.4 \mathrm{~Hz}, 1 \mathrm{H}), 2.45(\mathrm{dd}, J=13.8,9.6 \mathrm{~Hz}, 1 \mathrm{H}), 2.20-2.16(\mathrm{~m}, 1 \mathrm{H}), 1.82(\mathrm{~s}, 3 \mathrm{H}) ;{ }^{13} \mathrm{C}-\mathrm{NMR}$ $\left(150 \mathrm{MHz}, \mathrm{CDCl}_{3}\right) ; \delta=173.3,170.1,138.4,133.4,129.2,127.8,127.6,126.0,79.7,65.7,63.9,63.2,53.0,52.2,43.1$, 35.3, 17.0; IR (thin film) $v_{\max }=3448,3055,3023,2955,2883,1750,1600,1491,1436,1334,1274,1122,1076$; HRMS calc. for $\mathrm{C}_{19} \mathrm{H}_{23} \mathrm{NO}_{5}=345.1576$, exp. $=345.1577$. 


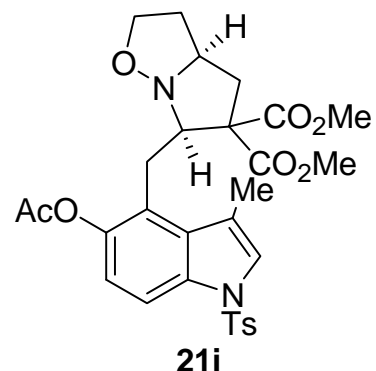

21i was prepared using General Procedure C for cis-adducts. The amounts of reagents employed were: cyclopropylalkoxylamine 17 (0.22 g, $1.0 \mathrm{mmol})$, indole-aldehyde $23^{7}(0.424 \mathrm{~g}, 1.10 \mathrm{mmol}), \mathrm{Yb}(\mathrm{OTf})_{3}(0.031 \mathrm{~g}, 0.05 \mathrm{mmol})$. White solid, $\mathrm{mp}=95-100{ }^{\circ} \mathrm{C}, 0.32 \mathrm{~g}(55 \%) ;{ }^{1} \mathrm{H}-\mathrm{NMR}\left(600 \mathrm{MHz}, \mathrm{CDCl}_{3}\right) ; \delta=7.83(\mathrm{~d}, J=8.4 \mathrm{~Hz}, 1 \mathrm{H}), 7.74(\mathrm{~d}, J=8.4 \mathrm{~Hz}$, 2H), $7.29(\mathrm{~s}, 1 \mathrm{H}), 7.21(\mathrm{~d}, J=8.4 \mathrm{~Hz}, 2 \mathrm{H}), 6.94(\mathrm{~d}, 8.4 \mathrm{~Hz}, 1 \mathrm{H}), 4.17(\mathrm{dd}, J=12.0,4.2 \mathrm{~Hz}, 1 \mathrm{H}), 3.83-3.76(\mathrm{~m}, 2 \mathrm{H}), 3.76$ (s, 3H), $3.73(\mathrm{~s}, 3 \mathrm{H}), 3.66-3.63(\mathrm{~m}, 1 \mathrm{H}), 3.47(\mathrm{dd}, J=16.2,7.8 \mathrm{~Hz}, 1 \mathrm{H}), 3.15(\mathrm{dd}, J=15.0,4.2 \mathrm{~Hz}, 1 \mathrm{H}), 2.75(\mathrm{dd}, J=$ 13.8, 5.4, 1H), 2.44-2.39 (m, 1H), 2.44 (s, 3H), 2.39 (s, 3H), 2.34 (s, 3H), 2.26 (dd, J=14.4, 10.2 Hz, 1H), $2.20-2.15$ (m, $1 \mathrm{H}) ;{ }^{13} \mathrm{C}-\mathrm{NMR}\left(150 \mathrm{MHz}, \mathrm{CDCl}_{3}\right) ; \delta=171.7,170.4,170.1,145.7,144.7,135.4,133.7,130.9,129.8,126.9,125.4,124.7$, $119.4,119.2,112.4,73.3,64.9,63.5,62.9,52.8,52.2,42.1,36.6,25.6,21.5,21.2,12.8 ;$ IR (thin film) $v_{\max }=3112,3055$, 2954, 2880, 1735, 1597, 1466, 1436, 1421, 1366, 1256, 1200, 1149, 1091; HRMS calc. for $\mathrm{C}_{29} \mathrm{H}_{32} \mathrm{~N}_{2} \mathrm{O}_{9} \mathrm{~S}=584.1829$, exp. $=584.1816$

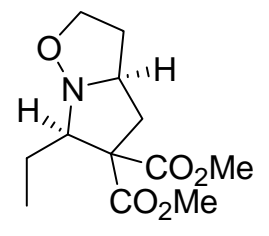

21j

21j was prepared using General Procedure C for cis-adducts. The amounts of reagents employed were: cyclopropylalkoxylamine 17 (0.22 g, $1.0 \mathrm{mmol})$, propionaldehyde ( $0.064 \mathrm{~g}, 1.10 \mathrm{mmol})$, Yb(OTf) $(0.031 \mathrm{~g}, 0.05 \mathrm{mmol})$. Colourless oil, $0.18 \mathrm{~g} \mathrm{(70 \% );}{ }^{1} \mathrm{H}-\mathrm{NMR}\left(400 \mathrm{MHz}, \mathrm{CDCl}_{3}\right) ; \delta=3.87$ (ddd, $\left.J=8.8,8.8,4.8 \mathrm{~Hz}, 1 \mathrm{H}\right), 3.77$ (dddd, $J=9.6,7.6,4.8$, $2.4 \mathrm{~Hz}, 1 \mathrm{H}), 3.70(\mathrm{~s}, 3 \mathrm{H}), 3.69(\mathrm{~s}, 3 \mathrm{H}), 3.69-3.62(\mathrm{~m}, 2 \mathrm{H}), 2.73(\mathrm{dd}, J=13.6,5.2 \mathrm{~Hz}, 1 \mathrm{H}), 2.54$ (dddd, $J=13.6,8.8,8.8$, $4.8 \mathrm{~Hz}, 1 \mathrm{H}), 2.28-2.20(\mathrm{~m}, 2 \mathrm{H}), 1.93-1.81(\mathrm{~m}, 2 \mathrm{H}), 1.09(\mathrm{t}, J=7.2 \mathrm{~Hz}, 3 \mathrm{H}) ;{ }^{13} \mathrm{C}-\mathrm{NMR}\left(100 \mathrm{MHz}, \mathrm{CDCl}_{3}\right) ; \delta=172.1$, $170.3,75.8,65.2,63.4,62.8,52.7,52.1,42.0,36.6,21.6,12.4$; IR (thin film) $v_{\max }=3630,3462,2955,2875,2362,1734$, 1701, 1437, 1250, 1162, 1130, 1089; HRMS calc. for $\mathrm{C}_{12} \mathrm{H}_{19} \mathrm{NO}_{5}=257.1263$, exp. $=257.1256$.

\footnotetext{
${ }^{7}$ For preparation of indole-aldehyde 23, see: England, D. B.; Kerr, M. A. J. Org. Chem. 2005, 70, 6519.
} 


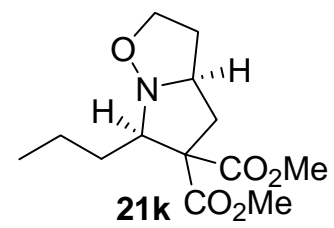

21k was prepared using General Procedure C for cis-adducts. The amounts of reagents employed were: cyclopropylalkoxylamine 17 (0.22 g, $1.0 \mathrm{mmol})$, butyraldehyde (0.079 g, $1.10 \mathrm{mmol}), \mathrm{Yb}(\mathrm{OTf})_{3}(0.031 \mathrm{~g}, 0.05 \mathrm{mmol})$. Colourless oil, $0.20 \mathrm{~g}(73 \%) ;{ }^{1} \mathrm{H}-\mathrm{NMR}\left(600 \mathrm{MHz}, \mathrm{CDCl}_{3}\right) ; \delta=3.88(\mathrm{ddd}, J=7.8,7.8,4.8 \mathrm{~Hz}, 1 \mathrm{H}), 3.78(\mathrm{dddd}, J=10.2,7.8,5.4$, $2.4 \mathrm{~Hz}, 1 \mathrm{H}), 3.75-3.66(\mathrm{~m}, 2 \mathrm{H}), 3.71(\mathrm{~s}, 6 \mathrm{H}), 2.73(\mathrm{dd}, J=13.2,4.8 \mathrm{~Hz}, 1 \mathrm{H}), 2.54(\mathrm{dddd}, J=16.8,8.4,8.4,4.8 \mathrm{~Hz}, 1 \mathrm{H})$, 2.30-2.21 (m, 2H), 1.89 (dddd, $J=19.2,9.6,9.6,4.8 \mathrm{~Hz}, 1 \mathrm{H}), 1.75$ (dddd, $J=19.2,9.6,9.6,4.8 \mathrm{~Hz}, 1 \mathrm{H}), 1.67-1.59$ (m, 1H), 1.49-1.41 (m, 1H), $0.96(\mathrm{t}, J=6.6 \mathrm{~Hz}, 3 \mathrm{H}) ;{ }^{13} \mathrm{C}-\mathrm{NMR}\left(150 \mathrm{MHz}, \mathrm{CDCl}_{3}\right) ; \delta=172.2,170.4,74.0,65.2,63.5,62.8$, 52.8, 52.2, 41.9, 36.6, 30.4, 21.1, 14.1; HRMS calc. for $\mathrm{C}_{13} \mathrm{H}_{21} \mathrm{NO}_{5}=271.1420$, exp. $=271.1414$.

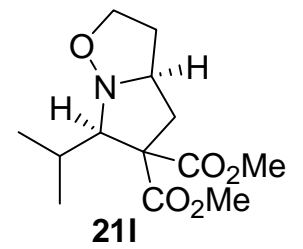

211 was prepared using General Procedure C for cis-adducts. The amounts of reagents employed were: cyclopropylalkoxylamine 17 (0.22 g, $1.0 \mathrm{mmol})$, isobutyraldehyde (0.079 g, $1.10 \mathrm{mmol}), \mathrm{Yb}(\mathrm{OTf})_{3}(0.031 \mathrm{~g}, 0.05 \mathrm{mmol})$. Colourless oil, 0.16 g (60 \%); ${ }^{1} \mathrm{H}-\mathrm{NMR}\left(600 \mathrm{MHz}, \mathrm{CDCl}_{3}\right) ; \delta=3.83-3.77(\mathrm{~m}, 2 \mathrm{H}), 3.71(\mathrm{~s}, 3 \mathrm{H}), 3.69(\mathrm{~s}, 3 \mathrm{H}), 3.61(\mathrm{ddd}, J=7.8,7.8$, $7.8 \mathrm{~Hz}, 1 \mathrm{H}), 3.32(\mathrm{~d}, J=10.2 \mathrm{~Hz}, 1 \mathrm{H}), 2.78(\mathrm{dd}, J=13.2,4.2 \mathrm{~Hz}, 1 \mathrm{H}), 2.52$ (dddd, $J=16.8,8.4,8.4,4.2,1 \mathrm{H}), 2.38$ (dddd, $J=13.2,10.8,7.2,7.2 \mathrm{~Hz}, 1 \mathrm{H}), 2.31(\mathrm{dd}, J=13.2,10.2 \mathrm{~Hz}, 1 \mathrm{H}), 2.23-2.01(\mathrm{~m}, 1 \mathrm{H}), 1.13(\mathrm{~d}, J=7.2 \mathrm{~Hz}, 3 \mathrm{H})$, $0.93(\mathrm{~d}, J=7.2 \mathrm{~Hz}, 3 \mathrm{H}) ;{ }^{13} \mathrm{C}-\mathrm{NMR}\left(150 \mathrm{MHz}, \mathrm{CDCl}_{3}\right) ; \delta=173.2,170.3,81.7,65.4,62.7,61.9,52.7,52.1,44.1,37.0$, 28.2, 22.0, 20.7; IR (thin film) $v_{\max }=2955,2875,1734,1457,1437,1249,1215,1195,1177 ; \mathrm{HRMS}$ calc. for $\mathrm{C}_{13} \mathrm{H}_{21} \mathrm{NO}_{5}$ $=271.1420$, exp. $=271.1423$. 


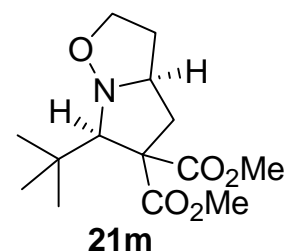

21m was prepared using General Procedure C for cis-adducts. The amounts of reagents employed were: cyclopropylalkoxylamine 17 (0.22 g, $1.0 \mathrm{mmol})$, trimethylacetaldehyde ( $0.095 \mathrm{~g}, 1.10 \mathrm{mmol}), \mathrm{Yb}(\mathrm{OTf})_{3}(0.031 \mathrm{~g}, 0.05 \mathrm{mmol})$. Colourless oil, $0.23 \mathrm{~g}(82 \%) ;{ }^{1} \mathrm{H}-\mathrm{NMR}\left(600 \mathrm{MHz}, \mathrm{CDCl}_{3}\right) ; \delta=3.91$ (add, $\left.J=14.4,7.8 \mathrm{~Hz}, 1 \mathrm{H}\right), 3.77-3.73(\mathrm{~m}, 2 \mathrm{H})$, $3.70(\mathrm{~s}, 3 \mathrm{H}), 3.69$ (s, 3H), $3.63(\mathrm{add}, J=16.2,7.8 \mathrm{~Hz}, 1 \mathrm{H}), 2.83(\mathrm{dd}, J=14.4,7.2 \mathrm{~Hz}, 1 \mathrm{H}), 2.48-2.42(\mathrm{~m}, 1 \mathrm{H}), 2.26(\mathrm{dd}$, $J=13.8,9.0 \mathrm{~Hz}, 1 \mathrm{H}), 2.12-2.07(\mathrm{~m}, 1 \mathrm{H}), 1.11(\mathrm{~s}, 9 \mathrm{H}) ;{ }^{13} \mathrm{C}-\mathrm{NMR}\left(150 \mathrm{MHz}, \mathrm{CDCl}_{3}\right) ; \delta=173.7,170.9,82.6,65.5,63.6$, $62.3,52.7,51.9,44.2,35.0,34.8,29.4$; IR (thin film) $v_{\max }=2955,2878,1731,1436,1251,1197,1175,1121,1058$, 1023; HRMS calc. for $\mathrm{C}_{14} \mathrm{H}_{23} \mathrm{NO}_{5}=285.1576$, exp. = 285.1566.; (+/-)-21m; white solid $\left(\mathrm{mp}=69-72{ }^{\circ} \mathrm{C}\right) ;(2 R, 5 R)-21 \mathrm{~m}$, colourless oil; $(2 \mathrm{R}, 5 \mathrm{R})-\mathbf{2 1 m},[\alpha]_{\mathrm{D}}^{22}=-11.5^{\circ}$ (c 3.9, $\left.\mathrm{CH}_{2} \mathrm{Cl}_{2}\right)$; GC/MS: $(2 S, 5 S: 2 R, 5 R$;)-21m, RT $=29.18,29.98$ $\min ;(2 R, 5 R)-21 \mathrm{~m}, \mathrm{RT}=29.98 \mathrm{~min}$, ee $=>99 \%$.

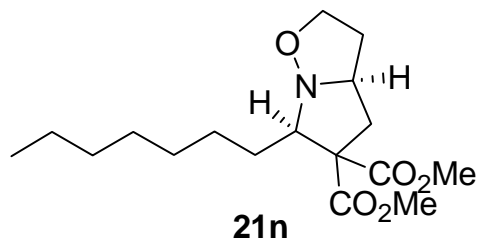

21n was prepared using General Procedure C for cis-adducts. The amounts of reagents employed were: cyclopropylalkoxylamine 17 (0.22 g, $1.0 \mathrm{mmol})$, octanal (0.141 g, $1.10 \mathrm{mmol})$, Yb(OTf) $3(0.031 \mathrm{~g}, 0.05 \mathrm{mmol})$. Colourless oil, 0.23 $\mathrm{g}(70 \%) ;{ }^{1} \mathrm{H}-\mathrm{NMR}\left(400 \mathrm{MHz}, \mathrm{CDCl}_{3}\right) ; \delta=3.87$ (ddd, $\left.J=8.8,8.8,4.8 \mathrm{~Hz}, 1 \mathrm{H}\right), 3.77$ (dddd, $\left.J=9.6,7.2,5.2,2.4 \mathrm{~Hz}, 1 \mathrm{H}\right)$, 3.73-3.64 (m, 2H), 3.699 (s, 3H), 3.696 (s, 3H), 2.72 (dd, $J=13.6,5.2 \mathrm{~Hz}, 1 \mathrm{H}), 2.54$ (dddd, $J=12.8,8.4,8.4,4.4 \mathrm{~Hz}$, 1H), 2.28-2.19 (m, 2H), 1.91-1.73 (m, 2H), 1.64-1.54 (m, 1H), 1.44-1.14 (m, 9H), $0.85(\mathrm{t}, J=6.8 \mathrm{~Hz}, 3 \mathrm{H})$; ${ }^{13} \mathrm{C}-\mathrm{NMR}$ $\left(100 \mathrm{MHz}, \mathrm{CDCl}_{3}\right) ; \delta=172.1,170.3,74.3,65.2,63.4,52.8,52.7,52.1,41.9,36.6,31.8,29.7,29.2,28.3,28.0,22.6$, 14.0; IR (thin film) $v_{\max }=3467,2956,2858,1733,1456,1435,1379,1337.1252,1208,1175,1127,1092,1062,1033$; HRMS calc. for $\mathrm{C}_{17} \mathrm{H}_{29} \mathrm{NO}_{5}=327.2046$, exp. $=327.2043$. 


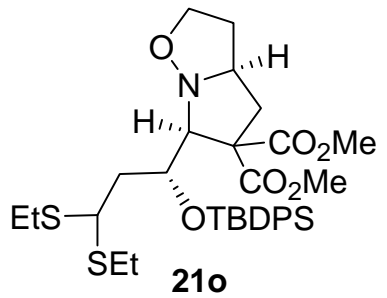

210 was using General Procedure $\mathrm{C}$ for cis-adducts. The amounts of reagents employed were: cyclopropylalkoxylamine $17(0.15 \mathrm{~g}, 0.70 \mathrm{mmol})$, aldehyde $18^{8}(0.31 \mathrm{~g}, 0.70 \mathrm{mmol}), \mathrm{Yb}(\mathrm{OTf})_{3}(0.02 \mathrm{~g}, 0.07 \mathrm{mmol})$. Waxy solid, 0.40 $\mathrm{g}(89 \%) ;{ }^{1} \mathrm{H}-\mathrm{NMR}\left(600 \mathrm{MHz}, \mathrm{CDCl}_{3}\right) ; \delta=7.86-7.85(\mathrm{~m}, 2 \mathrm{H}), 7.77-7.75(\mathrm{~m}, 2 \mathrm{H}), 7.40-7.37(\mathrm{~m}, 6 \mathrm{H}), 5.03(\mathrm{ddd}, J=7.8$, 7.8, 3.6 Hz, 1H), $4.11(\mathrm{dd}, J=9.6,5.4 \mathrm{~Hz}, 1 \mathrm{H}), 4.00(\mathrm{~d}, J=7.8 \mathrm{~Hz}, 1 \mathrm{H}), 3.78-3.70(\mathrm{~m}, 2 \mathrm{H}), 3.75(\mathrm{~s}, 3 \mathrm{H}), 3.61(\mathrm{~s}, 3 \mathrm{H})$, $3.50\left(\mathrm{ddd}, J_{1-3}=7.8 \mathrm{~Hz}, 1 \mathrm{H}\right), 2.84(\mathrm{dd}, J=13.2,4.8 \mathrm{~Hz}, 1 \mathrm{H}), 2.44(\mathrm{dddd}, J=12.0,8.4,8.4,4.2 \mathrm{~Hz}, 1 \mathrm{H}), 2.94-2.22(\mathrm{~m}$, 4H), 2.18-2.02 (m, 3H), 1.97 (dddd, $J=14.4,7.2,7.2,7.2 \mathrm{~Hz}, 1 \mathrm{H}), 1.01(\mathrm{t}, J=7.8 \mathrm{~Hz}, 3 \mathrm{H}), 0.95(\mathrm{t}, J=7.8 \mathrm{~Hz}, 3 \mathrm{H}), 0.93$ $(\mathrm{s}, 9 \mathrm{H}) ;{ }^{13} \mathrm{C}-\mathrm{NMR}\left(150 \mathrm{MHz}, \mathrm{CDCl}_{3}\right) ; \delta=172.1,170.2,135.7,135.6,135.1,133.8,129.4,129.1,127.4,127.3,80.1$, $71.0,65.2,63.1,62.0,52.60,52.58,52.1,47.9,45.0,43.9,36.3,26.4,23.7,23.0,19.1,14.3,14.1$; IR (thin film) $v_{\max }=$ 2958, 2931, 2858, 1739, 1454, 1430, 1258, 1175, 1110, 1061; HRMS calc. for $\mathrm{C}_{33} \mathrm{H}_{47} \mathrm{NO}_{6} \mathrm{~S}_{2} \mathrm{Si}=645.2614$, exp. $=645.2635 ;\left(2 S, 5 R, 1^{\prime} R\right)-210,[\alpha]_{\mathrm{D}}^{22}=+44.7^{\circ}\left(\mathrm{c} 10.2, \mathrm{CH}_{2} \mathrm{Cl}_{2}\right)$.

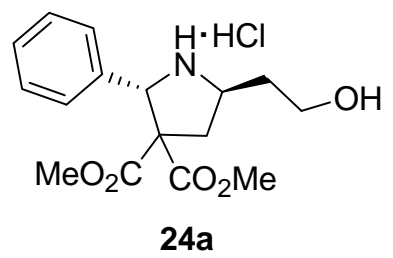

Compound 11a $(0.050 \mathrm{~g}, 0.164 \mathrm{mmol})$ and $\mathrm{Pd} / \mathrm{C}(10 \%$ on carbon, $0.005 \mathrm{~g})$ were dissolved in $1 \mathrm{~mL}$ of methanol and $\mathrm{AcCl}(0.023 \mathrm{~mL}, 0.328 \mathrm{mmol})$ was added. The flask was evacuated and purged with $\mathrm{H}_{2}$ through four cycles and finally placed under a balloon of $\mathrm{H}_{2}(1 \mathrm{~atm})$ and stirred for $16 \mathrm{hrs}$. The mixture was filtered through Celite and concentrated to yield salt 24a $(0.055 \mathrm{~g}, 0.049 \mathrm{mmol}, 98 \%)$ as a light green foam. ${ }^{1} \mathrm{H}-\mathrm{NMR}\left(600 \mathrm{MHz}, \mathrm{d}_{6}\right.$-DMSO); $\delta=10.61(\mathrm{broad}$ s, 1H), 9.72 (broad s, 1H), $7.41(\mathrm{~s}, 5 \mathrm{H}), 5.43(\mathrm{~s}, 1 \mathrm{H}), 4.32-4.28(\mathrm{~m}, 1 \mathrm{H}), 3.71(\mathrm{~s}, 3 \mathrm{H}), 3.58-3.51(\mathrm{~m}, 2 \mathrm{H}), 3.27(\mathrm{~s}, 3 \mathrm{H})$, $2.89(\mathrm{dd}, 1 \mathrm{H}, J=13.2,6.0 \mathrm{~Hz}), 2.29($ broad t, $1 \mathrm{H}, J=12.0 \mathrm{~Hz}), 2.08-2.02(\mathrm{~m}, 1 \mathrm{H}), 1.89-1.93(\mathrm{~m}, 1 \mathrm{H}) ;{ }^{13} \mathrm{C}-\mathrm{NMR}(100$ MHz, $\mathrm{d}_{6}$-DMSO); $\delta=168.6,168.3,132.1,129.3,128.4,128.2,64.3,63.8,57.3,57.1,53.3,52.7,38.1,34.5 ;$ IR (thin

${ }^{8}$ For preparation of aldehyde 18, see: a) Khanapure, S. P.; Kim, S.; Penrose, J. F.; Austen, F. K.; Powell, W. S.; Rokach, J. Tetrahedron Lett. 2002, 43, 6063-6066; b) Just, G.; Wang, Z. Y. J. Org. Chem. 1986, 51, 4796-4802. 


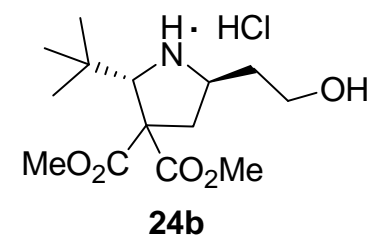

Compound 11j $(0.051 \mathrm{~g}, 0.175 \mathrm{mmol})$ and $\mathrm{Pd} / \mathrm{C}(10 \%$ on carbon, $0.005 \mathrm{~g})$ were dissolved in $1 \mathrm{~mL}$ of methanol and $\mathrm{AcCl}$ $(0.025 \mathrm{~mL}, 0.350 \mathrm{mmol})$ was added. The flask was evacuated and purged with $\mathrm{H}_{2}$ through four cycles and finally placed under a balloon of $\mathrm{H}_{2}(1 \mathrm{~atm})$ and stirred for $16 \mathrm{hrs}$. The mixture was filtered through Celite and concentrated to yield salt 24b (0.056 g, $0.051 \mathrm{mmol}$, quantitative yield) as a light yellow foam. ${ }^{1} \mathrm{H}-\mathrm{NMR}\left(600 \mathrm{MHz}, \mathrm{d}_{6}\right.$-DMSO); $\delta=10.11$ (broad s, 1H), 9.03 (broad s, 1H), $4.23-4.17(\mathrm{~m}, 1 \mathrm{H}), 4.10-4.01(\mathrm{~m}, 1 \mathrm{H}), 3.73(\mathrm{~s}, 3 \mathrm{H}), 3.71(\mathrm{~s}, 3 \mathrm{H}), 3.57-3.51(\mathrm{~m}$, $1 \mathrm{H}), 3.51-3.46(\mathrm{~m}, 1 \mathrm{H}), 2.72-2.67(\mathrm{~m}, 1 \mathrm{H}), 2.10-2.01(\mathrm{~m}, 1 \mathrm{H}), 1.97(\mathrm{t}, J=12.6 \mathrm{~Hz}, 1 \mathrm{H}), 1.86-1.79(\mathrm{~m}, 1 \mathrm{H}), 1.02$ (s, 9H); ${ }^{13} \mathrm{C}-\mathrm{NMR}\left(100 \mathrm{MHz}, \mathrm{d}_{6}\right.$-DMSO); $\delta=169.6,169.3,70.5,59.6,57.4,56.9,53.3,53.0,41.4,34.4,33.4,26.7 ; \mathrm{IR}$ (thin film) $v_{\max }=3421,2958,1734,1662,1647,1437,1212,1186,1101,1061,746$; HRMS calc. for $\mathrm{C}_{14} \mathrm{H}_{27} \mathrm{NO}_{5}{ }^{[2+]}=$ 289.1878, exp. $=289.1903$.

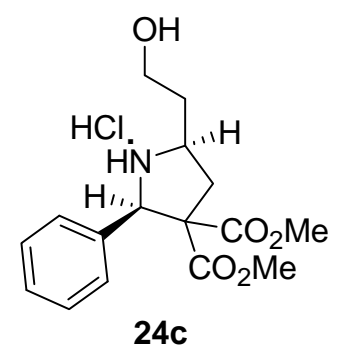

To a solution of adduct 21a $(0.17 \mathrm{~g}, 0.56 \mathrm{mmol})$ in $\mathrm{MeOH}(20 \mathrm{ml})$ was added $\mathrm{AcCl}(0.08 \mathrm{ml}, 1.12 \mathrm{mmol}) \mathrm{and} \mathrm{Pd} / \mathrm{C}$ $(0.217 \mathrm{~g}$ of $10 \mathrm{wt} \% \mathrm{Pd} / \mathrm{C})$. The flask was evacuated and purged with $\mathrm{H}_{2}$ through 5 cycles and finally pressurized with $\mathrm{H}_{2}$ to 65 psi. The flask was shaken for $18 \mathrm{hrs}$ after which the solution was filtered though Celite, rinsed with $\mathrm{MeOH}$ and concentrated in vacuo to afford $0.19 \mathrm{~g}(100 \%)$ of pyrrolidine salt $24 \mathrm{c}$ as a deliquescent white solid; ${ }^{1} \mathrm{H}-\mathrm{NMR}(600 \mathrm{MHz}$, $\mathrm{d}_{6}$-DMSO): $\delta=10.96$ (br s, 1H), 9.02 (br m, 1H), 7.47-7.42 (m, $\left.5 \mathrm{H}\right), 5.32$ (br m, 1H), 3.75 (s, 3H), 3.75-3.71 (m, 2H), $3.55(\mathrm{t}, J=5.4 \mathrm{~Hz}, 2 \mathrm{H}), 3.17(\mathrm{~s}, 3 \mathrm{H}), 2.78-2.69(\mathrm{~m}, 2 \mathrm{H}), 2.07(\mathrm{ddd}, J=13.2,13.2,6.6 \mathrm{~Hz}, 1 \mathrm{H}), 1.96(\mathrm{ddd}, J=13.2,13.2$, $6.6 \mathrm{~Hz}, 1 \mathrm{H}) ;{ }^{13} \mathrm{C}-\mathrm{NMR}\left(100 \mathrm{MHz}, \mathrm{CD}_{3} \mathrm{OD}\right): \delta=170.9,169.5,132.7,131.0,130.0,129.3,67.6,64.5,59.6,58.6,54.4$, 


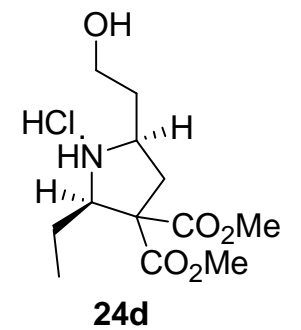

To a solution of adduct $21 \mathbf{j}(0.135 \mathrm{~g}, 0.52 \mathrm{mmol})$ in $\mathrm{MeOH}$ was added $\mathrm{AcCl}(0.075 \mathrm{ml}, 1.05 \mathrm{mmol})$ and $\mathrm{Pd} / \mathrm{C}(0.135 \mathrm{~g}$ of 10 wt $\% \mathrm{Pd} / \mathrm{C})$. The flask was evacuated and purged with $\mathrm{H}_{2}$ through 5 cycles and finally pressurized with $\mathrm{H}_{2}$ to 65 psi. The flask was shaken for $18 \mathrm{hrs}$ after which the solution was filtered though Celite, rinsed with MeOH and concentrated in vacuo to afford $0.16 \mathrm{~g}(100 \%)$ of pyrrolidine salt $\mathbf{2 4 d}$ as a deliquescent white solid. ${ }^{1} \mathrm{H}-\mathrm{NMR}\left(600 \mathrm{MHz}, \mathrm{d}_{6}-\mathrm{DMSO}\right)$; $\delta=10.67($ br s, $1 \mathrm{H}), 8.79($ br s, $1 \mathrm{H}), 4.79($ br s, $1 \mathrm{H}), 3.93($ br m, $1 \mathrm{H}), 3.72(\mathrm{~s}, 6 \mathrm{H}), 3.54-3.48(\mathrm{~m}, 3 \mathrm{H}), 2.66(\mathrm{dd}, J=14.4$, $8.4 \mathrm{~Hz}, 1 \mathrm{H}), 2.41(\mathrm{dd}, J=14.4,8.4 \mathrm{~Hz}, 1 \mathrm{H}), 1.97-1.92(\mathrm{~m}, 1 \mathrm{H}), 1.83-1.78(\mathrm{~m}, 1 \mathrm{H}), 1.72-1.67(\mathrm{~m}, 1 \mathrm{H}), 1.58-1.51(\mathrm{~m}, 1 \mathrm{H})$, $1.01(\mathrm{t}, J=7.2 \mathrm{~Hz}, 3 \mathrm{H}) ;{ }^{13} \mathrm{C}-\mathrm{NMR}\left(100 \mathrm{MHz}, \mathrm{CD}_{3} \mathrm{OD}\right): \delta=170.7,66.3,62.3,59.6,57.8,54.1,54.0,39.0,35.3,23.3$, 11.8; IR (thin film) $v_{\max }=3388,2957,2757,1735,1437,1281,1215,1085,1059$; HRMS calc. for $\mathrm{C}_{12} \mathrm{H}_{22} \mathrm{NO}_{5}{ }^{++}=$ 260.1498, exp. $=260.1500$.

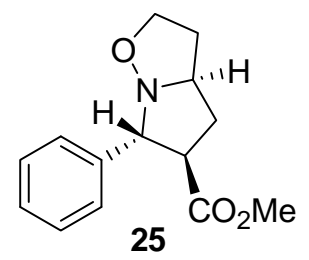

Compound 11a $(0.052 \mathrm{~g}, 0.17 \mathrm{mmol})$, sodium cyanide $(0.019 \mathrm{~g}, 0.38 \mathrm{mmol})$, and water $(0.007 \mathrm{~g}, 0.38 \mathrm{mmol})$ were dissolved in $2.6 \mathrm{~mL}$ of DMSO and heated to $140{ }^{\circ} \mathrm{C}$ for $15 \mathrm{~min}$ in a microwave reactor. The reaction mixture was partitioned between EtOAc and $\mathrm{H}_{2} \mathrm{O}$. The aqueous layer was removed and the organic layer washed with water (2x). The combined aqueous extracts were washed once with EtOAc and the combined organic extracts were then washed with brine (1x), dried over $\mathrm{MgSO}_{4}$, pre-absorbed onto silica and purified by flash column chromatography. A $1: 0.14$ mixture 
of isomers were observed in the crude mixture by ${ }^{1} \mathrm{H}$ NMR. Compound $25^{9}(0.031 \mathrm{~g}, 0.12 \mathrm{mmol}, 72 \%, 76 \%$ brsm $)$ was isolated as a white solid, and its minor isomer ( $0.006 \mathrm{~g}, 0.02 \mathrm{mmol}, 13 \%, 14 \% \mathrm{brsm})$ as a colourless oil (85 \% overall yield, $89 \%$ brsm). Major isomer 25: $\mathrm{mp}=72-75{ }^{\circ} \mathrm{C} ;{ }^{1} \mathrm{H}-\mathrm{NMR}\left(600 \mathrm{MHz}, \mathrm{CDCl}_{3}\right) ; \delta=7.42(\mathrm{~d}, 2 \mathrm{H}, \mathrm{J}=7.8 \mathrm{~Hz}), 7.32(\mathrm{t}$, $2 \mathrm{H}, J=7.8 \mathrm{~Hz}), 7.26(\mathrm{t}, 1 \mathrm{H}, J=7.8 \mathrm{~Hz}), 4.34(\mathrm{~d}, 1 \mathrm{H}, J=10.8 \mathrm{~Hz}), 4.09(\mathrm{ddd}, 1 \mathrm{H}, J=7.8,7.2,7.2 \mathrm{~Hz}), 3.97-3.92(\mathrm{~m}$, 2H), $3.63(\mathrm{~s}, 3 \mathrm{H}), 3.00(\mathrm{ddd}, 1 \mathrm{H}, J=9.6,9.6,7.2 \mathrm{~Hz}), 2.50-2.40(\mathrm{~m}, 2 \mathrm{H}), 2.15-2.10(\mathrm{~m}, 1 \mathrm{H}), 2.06(\mathrm{ddd}, 1 \mathrm{H}, J=13.2$, 10.2, 7.2 Hz); ${ }^{13} \mathrm{C}-\mathrm{NMR}\left(100 \mathrm{MHz}, \mathrm{CDCl}_{3}\right) ; \delta=173.3,139.9,128.4,127.6,127.2,71.4,64.9,62.8,52.0,50.3,36.4$, 34.4; IR (thin film) $v_{\max }=3031,2953,2877,1734,1496,1455,1437,1364,1294,1198,1170,1029,983,751,701$; HRMS calc. for $\mathrm{C}_{14} \mathrm{H}_{17} \mathrm{NO}_{3}=247.1213$, exp. $=247.1208$.

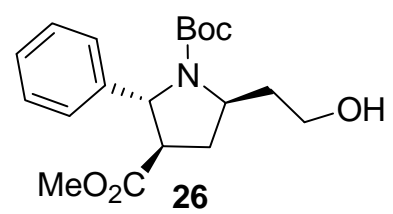

Compound $25(0.038 \mathrm{~g}, 0.16 \mathrm{mmol}), \mathrm{Boc}_{2} \mathrm{O}(0.068 \mathrm{~g}, 0.31 \mathrm{mmol})$, and $\mathrm{Pd}(\mathrm{OH})_{2} / \mathrm{C}(20 \%$ on carbon, $0.038 \mathrm{~g})$, were dissolved in $1 \mathrm{~mL}$ of methanol. The flask was evacuated and purged with $\mathrm{H}_{2}$ through four cycles and finally placed under a balloon of $\mathrm{H}_{2}(1 \mathrm{~atm})$ and stirred for $16 \mathrm{hrs}$. The reaction mixture was filtered through Celite, concentrated, preabsorbed onto silica and purified by flash column chromatography. Compound 26 (0.042 g, 0.12 mmol, $78 \%$ was obtained as a colourless oil. ${ }^{1} \mathrm{H}-\mathrm{NMR}\left(600 \mathrm{MHz}, \mathrm{CDCl}_{3}\right) ; \delta=7.30(\mathrm{t}, 2 \mathrm{H}, J=7.2 \mathrm{~Hz}), 7.23(\mathrm{t}, 1 \mathrm{H}, J=7.2 \mathrm{~Hz}), 7.14(\mathrm{~d}$, $2 \mathrm{H}, J=7.8 \mathrm{~Hz}), 5.30(\mathrm{~s}, 1 \mathrm{H}), 4.43(\operatorname{broad} \mathrm{q}, J=7.2 \mathrm{~Hz}), 3.76(\mathrm{~s}, 3 \mathrm{H}), 3.67-3.60(\mathrm{~m}, 3 \mathrm{H}), 2.85(\mathrm{~d}, 1 \mathrm{H}, J=8.4 \mathrm{~Hz}), 2.55$ $(\mathrm{ddd}, 1 \mathrm{H}, J=13.2,8.4,8.4 \mathrm{~Hz}), 2.15(\mathrm{~d}, 1 \mathrm{H}, J=13.2 \mathrm{~Hz}), 1.77-1.67(\mathrm{~m}, 2 \mathrm{H}), 1.07(\mathrm{~s}, 9 \mathrm{H}) ;{ }^{13} \mathrm{C}-\mathrm{NMR}(100 \mathrm{MHz}$, $\left.\mathrm{CDCl}_{3}\right) ; \delta=174.0,155.2,144.3,128.4,127.0,125.0,80.1,63.8,59.4,54.9,52.4,51.3,38.7,31.4,27.8 ;$ IR (thin film) $v_{\max }=3448$ (broad), 2975, 2954, 2932, 2885, 1738, 1685, 1675, 1456, 1394, 1367, 1250, 1168, 1128, 1095, 1070, 1030, 960, 913,88, 856, 774,736, 703, 649; HRMS calc. for $\mathrm{C}_{19} \mathrm{H}_{27} \mathrm{NO}_{5}=349.1889$, exp. $=349.1889$.

\footnotetext{
${ }^{9}$ Stereochemical assignment based on 1D NOE experiments.
} 


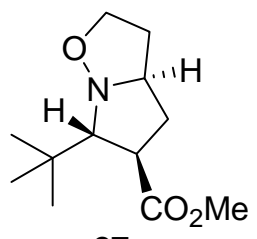

27

Compound 11j $(0.129 \mathrm{~g}, 0.45 \mathrm{mmol})$, sodium cyanide $(0.049 \mathrm{~g}, 1.00 \mathrm{mmol})$, and water $(0.018 \mathrm{~g}, 1.00 \mathrm{mmol})$ were dissolved in $5 \mathrm{~mL}$ of DMSO and heated to $140{ }^{\circ} \mathrm{C}$ for $15 \mathrm{~min}$ in a microwave reactor. The reaction mixture was partitioned between EtOAc and $\mathrm{H}_{2} \mathrm{O}$. The organic layer was washed with water $(2 \mathrm{X})$. The combined aqueous extracts was washed once with EtOAc. The combined organic extracts were then washed with brine, dried over $\mathrm{MgSO}_{4}$, $\mathrm{pre}^{-}$ absorbed onto silica and purified by flash column chromatography. Compound 27 (0.085 g, 0.37 mmol, $83 \%)$ was obtained as a single diastereomer. ${ }^{1} \mathrm{H}-\mathrm{NMR}\left(600 \mathrm{MHz}, \mathrm{CDCl}_{3}\right) ; \delta=3.92(\mathrm{ddd}, 1 \mathrm{H}, J=8.4,8.4,6.6 \mathrm{~Hz}), 3.83(\mathrm{ddd}, 1 \mathrm{H}, J$ $=8.4,8.4,4.2 \mathrm{~Hz}), 3.67(\mathrm{~s}, 3 \mathrm{H}), 3.67-3.62(\mathrm{~m}, 1 \mathrm{H}), 3.27(\mathrm{~d}, 1 \mathrm{H}, J=7.8 \mathrm{~Hz}), 2.79(\mathrm{ddd}, 1 \mathrm{H}, J=9.6,7.8,7.8 \mathrm{~Hz}), 2.31$ $(\mathrm{dq}, J=12.0,8.4 \mathrm{~Hz}), 2.24-2.19(\mathrm{~m}, 1 \mathrm{H}), 2.01$ (dddd, 1H, $J=12.6,7.2,4.8,3.0 \mathrm{~Hz}), 1.91(\mathrm{ddd}, 1 \mathrm{H}, J=12.6,9.0,9.0$ $\mathrm{Hz}), 0.92$ (s, 9H); ${ }^{13} \mathrm{C}-\mathrm{NMR}\left(100 \mathrm{MHz}, \mathrm{CDCl}_{3}\right) ; \delta=175.4,78.8,64.4,64.3,52.0,44.9,36.0,35.5,33.9,26.6$; $1 \mathrm{D}$ NOE correlation: H3 (2.79 ppm) - H5 (3.67-3.62 ppm); IR (thin film) $v_{\max }=2953,2870,1735,1457,1436,1394,1365,1267$, 1202, 1168, 1113, 1088, 1018; HRMS calc. for $\mathrm{C}_{12} \mathrm{H}_{21} \mathrm{NO}_{3}=227.1520$, exp $=227.1521$.

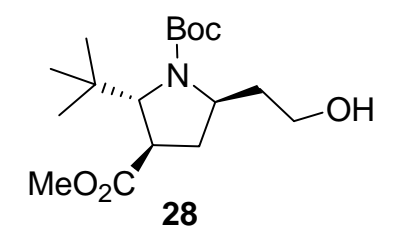

Compound 27 (0.062 g, $0.27 \mathrm{mmol}), \mathrm{Boc}_{2} \mathrm{O}(0.110 \mathrm{~g}, 0.50 \mathrm{mmol})$, and palladium hydroxide (20\% on carbon, $\left.0.012 \mathrm{~g}\right)$, were dissolved in $4 \mathrm{~mL}$ of methanol. The flask was evacuated and purged with $\mathrm{H}_{2}$ through four cycles and finally placed under a balloon of $\mathrm{H}_{2}(1 \mathrm{~atm})$ and stirred for $16 \mathrm{hrs}$. The reaction mixture was filtered through Celite, concentrated, preabsorbed onto silica and purified by flash column chromatography. Compound 28 (0.077 g, 0.23 mmol, $86 \%)$ was obtained as a colourless oil. ${ }^{1} \mathrm{H}-\mathrm{NMR}\left(600 \mathrm{MHz}, \mathrm{CDCl}_{3}\right) ; \delta=4.26(\mathrm{~s}, 1 \mathrm{H}), 3.90(\mathrm{dddd}, 1 \mathrm{H}, J=8.4,8.4,3.0,3.0 \mathrm{~Hz}), 3.72$ (s, 3H), 3.69 (q, 1H, $J=5.4 \mathrm{~Hz}), 3.62(\mathrm{ddd}, 1 \mathrm{H}, J=11.4,8.4,4.2 \mathrm{~Hz}), 2.89$ (d, 1H, $J=9.0 \mathrm{~Hz}), 2.37$ (ddd, $1 \mathrm{H}, J=13.8$, 9.0, $9.0 \mathrm{~Hz}), 2.31$ (dddd, 1H, $J=14.4,8.4,5.4,3.0 \mathrm{~Hz}), 2.20(\mathrm{~d}, 1 \mathrm{H}, J=13.8 \mathrm{~Hz}), 1.82$ (broad s, $1 \mathrm{H}), 1.63$ (dddd, $1 \mathrm{H}, J$ $=13.2,8.4,4.8,4.8 \mathrm{~Hz}), 1.46(\mathrm{~s}, 9 \mathrm{H}), 0.92(\mathrm{~s}, 9 \mathrm{H}) ;{ }^{13} \mathrm{C}-\mathrm{NMR}\left(100 \mathrm{MHz}, \mathrm{CDCl}_{3}\right) ; \delta=175.4,155.1,79.8,68.4,60.7,56.7$, $52.2,43.9,37.4,36.9,33.6,28.3,27.6$; IR (thin film) $v_{\max }=3446$ (broad), 2974, 2877, 1739, 1700, 1695, 1684, 1481, $1467,1367,1170,1124,1096,1067,1032,963,912,863,781,734$; HRMS calc. for $\mathrm{C}_{17} \mathrm{H}_{32} \mathrm{NO}_{5}\left[\mathrm{H}^{+}\right]=330.2283$, exp. $=$ 330.2275 . 


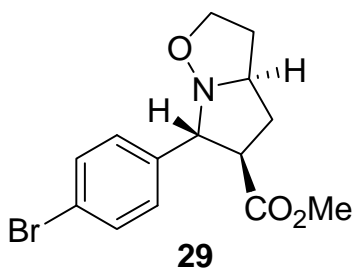

Compound 11b (0.051 g, $0.13 \mathrm{mmol})$, sodium cyanide $(0.014 \mathrm{~g}, 0.29 \mathrm{mmol})$, and water $(0.005 \mathrm{~g}, 0.29 \mathrm{mmol})$ were dissolved in $2 \mathrm{~mL}$ of DMSO and heated to $140{ }^{\circ} \mathrm{C}$ for $15 \mathrm{~min}$ in a microwave reactor. The reaction mixture was partitioned between EtOAc and $\mathrm{H}_{2} \mathrm{O}$. The organic layer was washed with water (2x), the combined aqueous extracts was washed once with EtOAc. The combined organic extracts were then washed with brine (1x), dried over $\mathrm{MgSO}_{4}$, preabsorbed onto silica and purified by flash column chromatography. A $1: 0.15$ mixture of isomers were observed in the crude mixture by ${ }^{1} \mathrm{H}$ NMR. Compound $\mathbf{2 9}^{\mathbf{1 0}}(0.033 \mathrm{~g}, 0.10 \mathrm{mmol}, 77 \%)$ was isolated as a white solid, and its isomer (0.005 g, $0.02 \mathrm{mmol}, 13 \%)$, as a colourless oil (90\% overall yield). Major isomer 29: ${ }^{1} \mathrm{H}-\mathrm{NMR}\left(600 \mathrm{MHz}, \mathrm{CDCl}_{3}\right) ; \delta=$ $7.45(\mathrm{~d}, 2 \mathrm{H}, J=7.8 \mathrm{~Hz}), 7.31(\mathrm{~d}, 2 \mathrm{H}, J=9 \mathrm{~Hz}), 4.26(\mathrm{~d}, 1 \mathrm{H}, J=10.2 \mathrm{~Hz}), 4.07(\mathrm{ddd}, 1 \mathrm{H}, J=9.0,9.0,7.2 \mathrm{~Hz}), 3.97-$ $3.91(\mathrm{~m}, 2 \mathrm{H}), 3.63(\mathrm{~s}, 3 \mathrm{H}), 2.93(\mathrm{ddd}, 1 \mathrm{H}, J=10.8,10.8,7.8 \mathrm{~Hz}), 2.48$ (ddd, $J=13.2,7.8,7.8 \mathrm{~Hz}), 2.42$ (dddd, $1 \mathrm{H}, J=$ 12.0, 8.4, 8.4, 8.4 Hz), $2.13-2.09(\mathrm{~m}, 1 \mathrm{H}), 2.03(\mathrm{ddd}, 1 \mathrm{H}, J=12.6,10.8,7.2 \mathrm{~Hz}) ;{ }^{13} \mathrm{C}-\mathrm{NMR}\left(100 \mathrm{MHz}, \mathrm{CDCl}_{3}\right) ; \delta=$ $172.9,139.0,131.6,129.0,121.6,70.7,65.0,62.7,52.1,50.3,36.4,34.4$; IR (thin film) $v_{\max }=2952,1734,1489,1437$, 1263, 1170, 1011, 914, 812, 744; HRMS calc. for $\mathrm{C}_{14} \mathrm{H}_{16} \mathrm{BrNO}_{3}=325.0312$, exp. $=325.0314$.

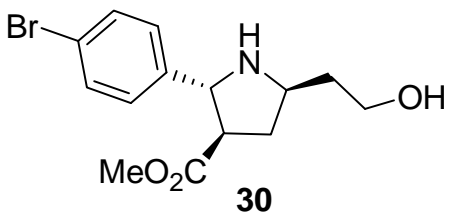

Compound $29(0.009 \mathrm{~g}, 0.03 \mathrm{mmol})$ was dissolved in $0.5 \mathrm{~mL}$ of acetic acid. Zinc dust $(0.013 \mathrm{~g}, 0.19 \mathrm{mmol})$ was added to the solution and stirred at room temperature for 16 hours. The reaction was filtered through Celite, concentrated, neutralized with a saturated solution of $\mathrm{NaHCO}_{3}$, and extracted with EtOAc to yield compound $30(0.005 \mathrm{~g}, 0.016 \mathrm{mmol}$, $58 \%$ ) as a light yellow oil. ${ }^{1} \mathrm{H}-\mathrm{NMR}\left(600 \mathrm{MHz}, \mathrm{CDCl}_{3}\right) ; \delta=7.45(\mathrm{~d}, 2 \mathrm{H}, J=7.8 \mathrm{~Hz}), 7.23(\mathrm{~d}, 2 \mathrm{H}, J=7.8 \mathrm{~Hz}), 4.48(\mathrm{~d}$, $1 \mathrm{H}, J=7.8 \mathrm{~Hz}), 3.85-3.76(\mathrm{~m}, 2 \mathrm{H}), 3.68(\mathrm{~s}, 3 \mathrm{H}), 3.61-3.57(\mathrm{~m}, 1 \mathrm{H}), 2.95(\mathrm{q}, 1 \mathrm{H}, J=9.0 \mathrm{~Hz}), 2.71(\mathrm{broad} \mathrm{s}, 2 \mathrm{H}), 2.44$ $(\mathrm{ddd}, 1 \mathrm{H}, J=12.6,7.8,7.8 \mathrm{~Hz}), 1.89(\mathrm{ddd}, 1 \mathrm{H}, J=12.6,9.0,9.0 \mathrm{~Hz}), 1.85-1.76(\mathrm{~m}, 2 \mathrm{H}) ;{ }^{13} \mathrm{C}-\mathrm{NMR}(100 \mathrm{MHz}$ $\left.\mathrm{CDCl}_{3}\right) ; \delta=174.2,140.4,131.8,128.3,121.4,64.2,62.0,28.8,52.2,52.1,38.1,36.8 ;$ IR (thin film) $v_{\max }=3336(\mathrm{broad})$, $2951,2930,2875,1734,1559,1489,1437,1369,1272,1171,1072,1010,913,821,734$; HRMS calc. for $\mathrm{C}_{14} \mathrm{H}_{18} \mathrm{BrNO}_{3}$ $=327.0465$, exp. $=327.0470$.

\footnotetext{
${ }^{10}$ Stereochemical assignment based on 1D NOE experiments.
} 


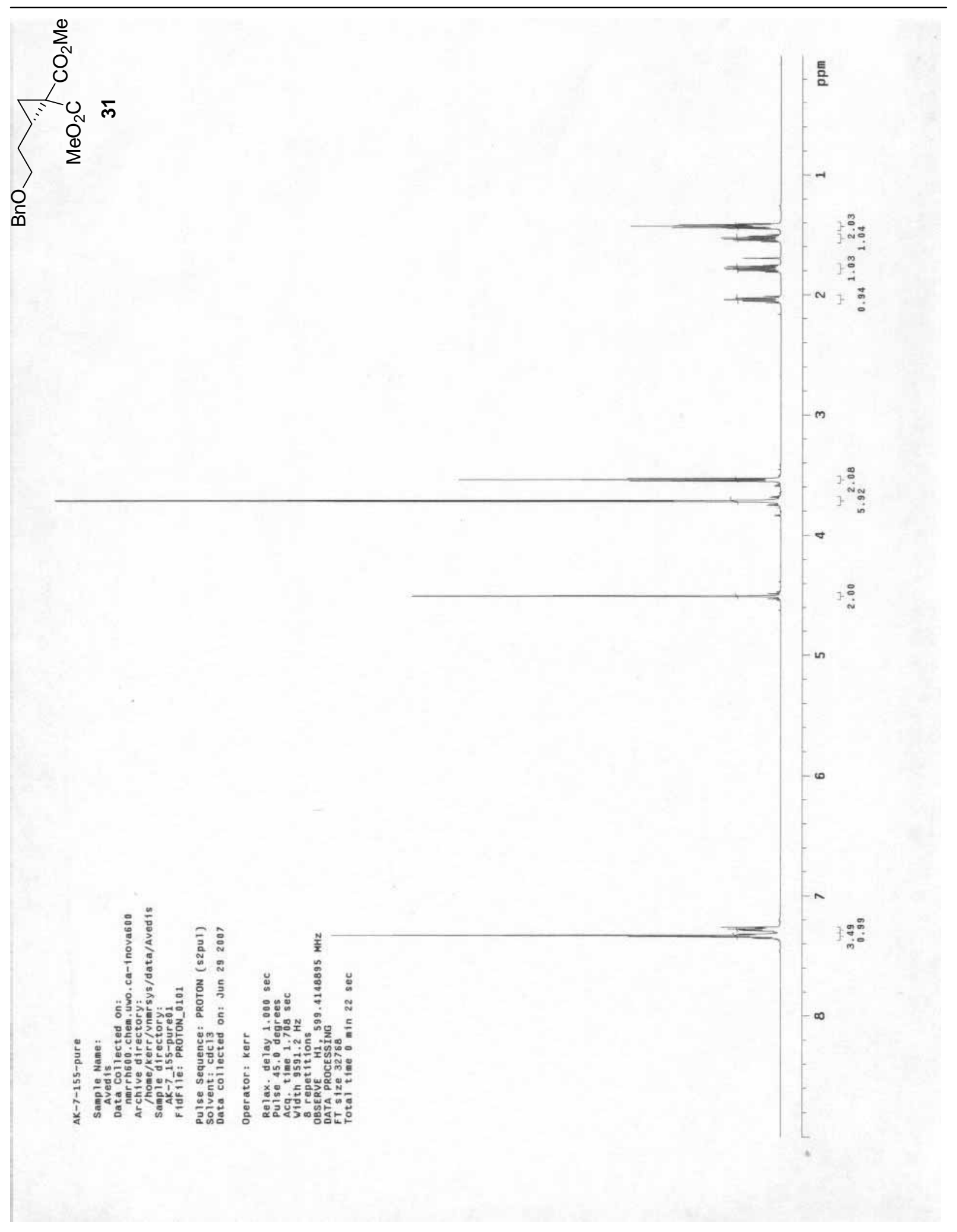




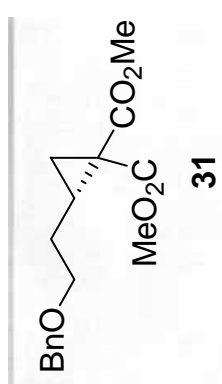

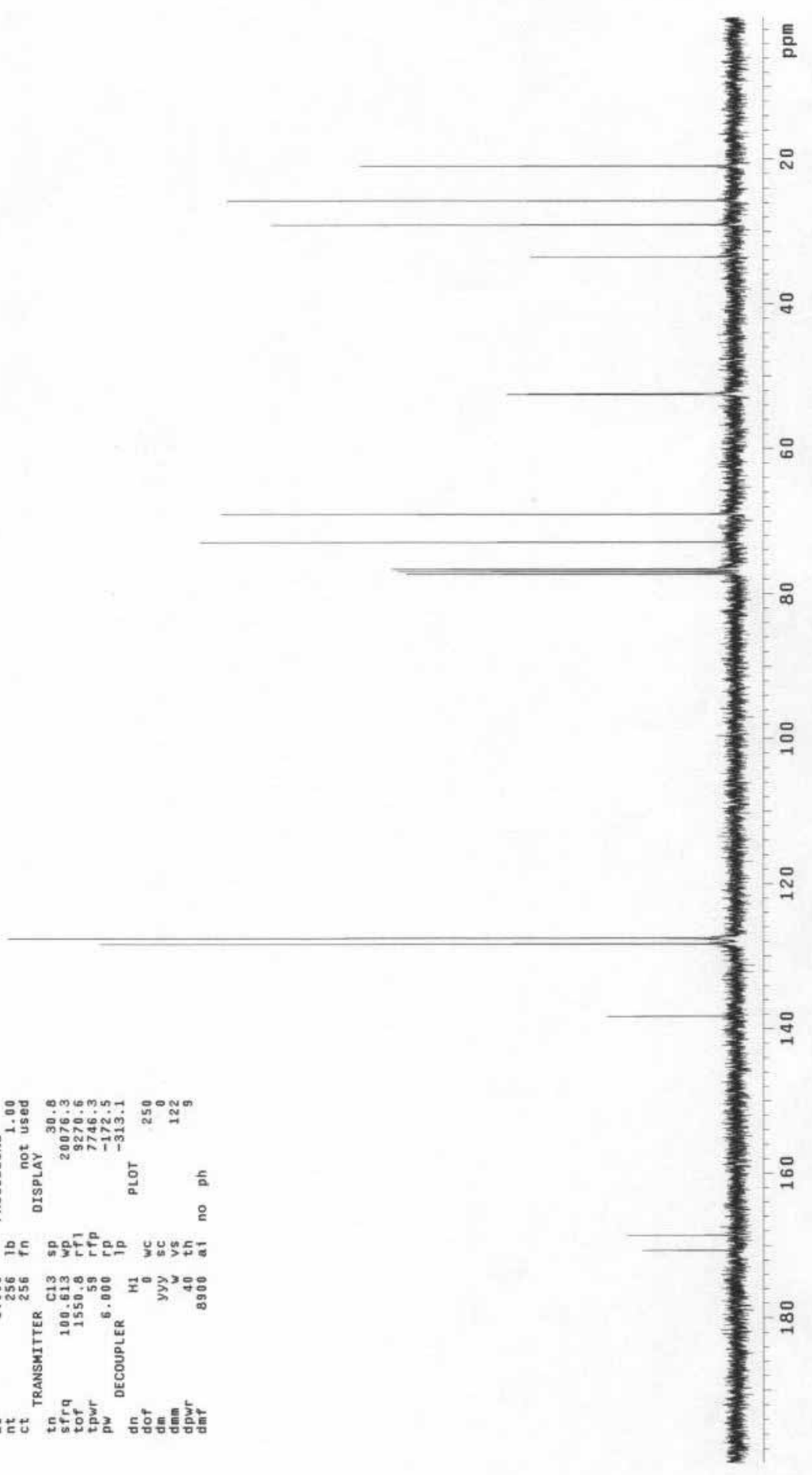




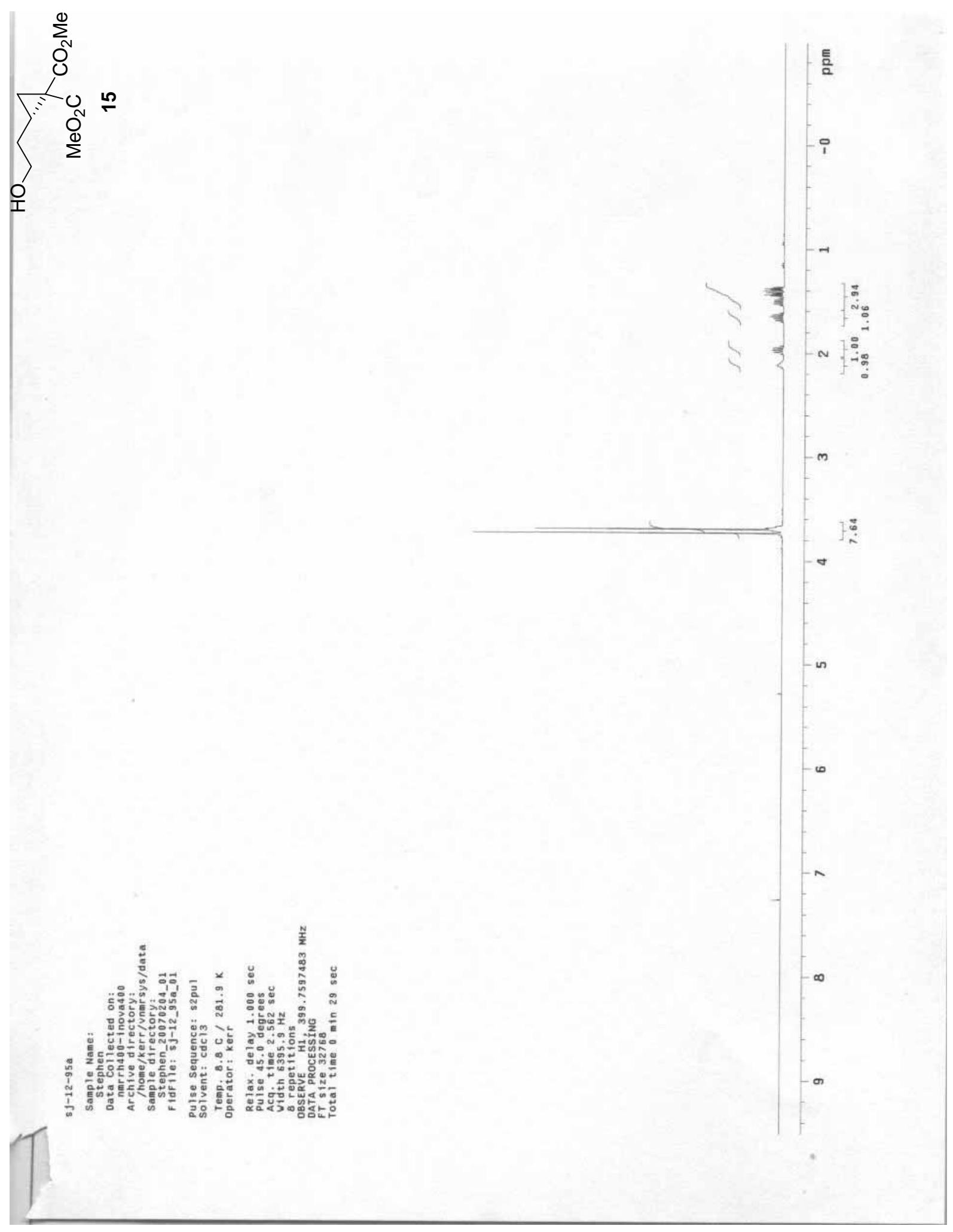



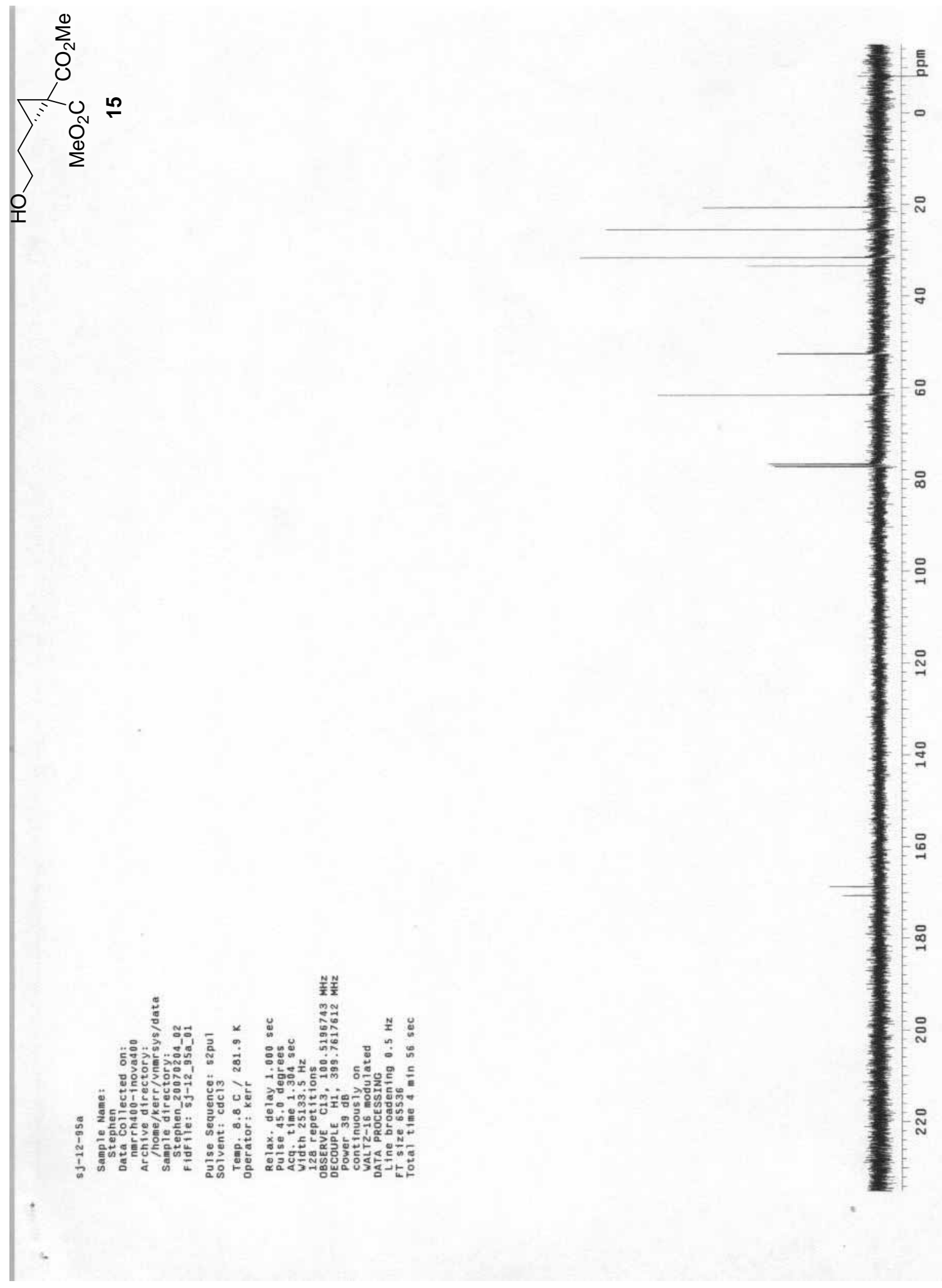

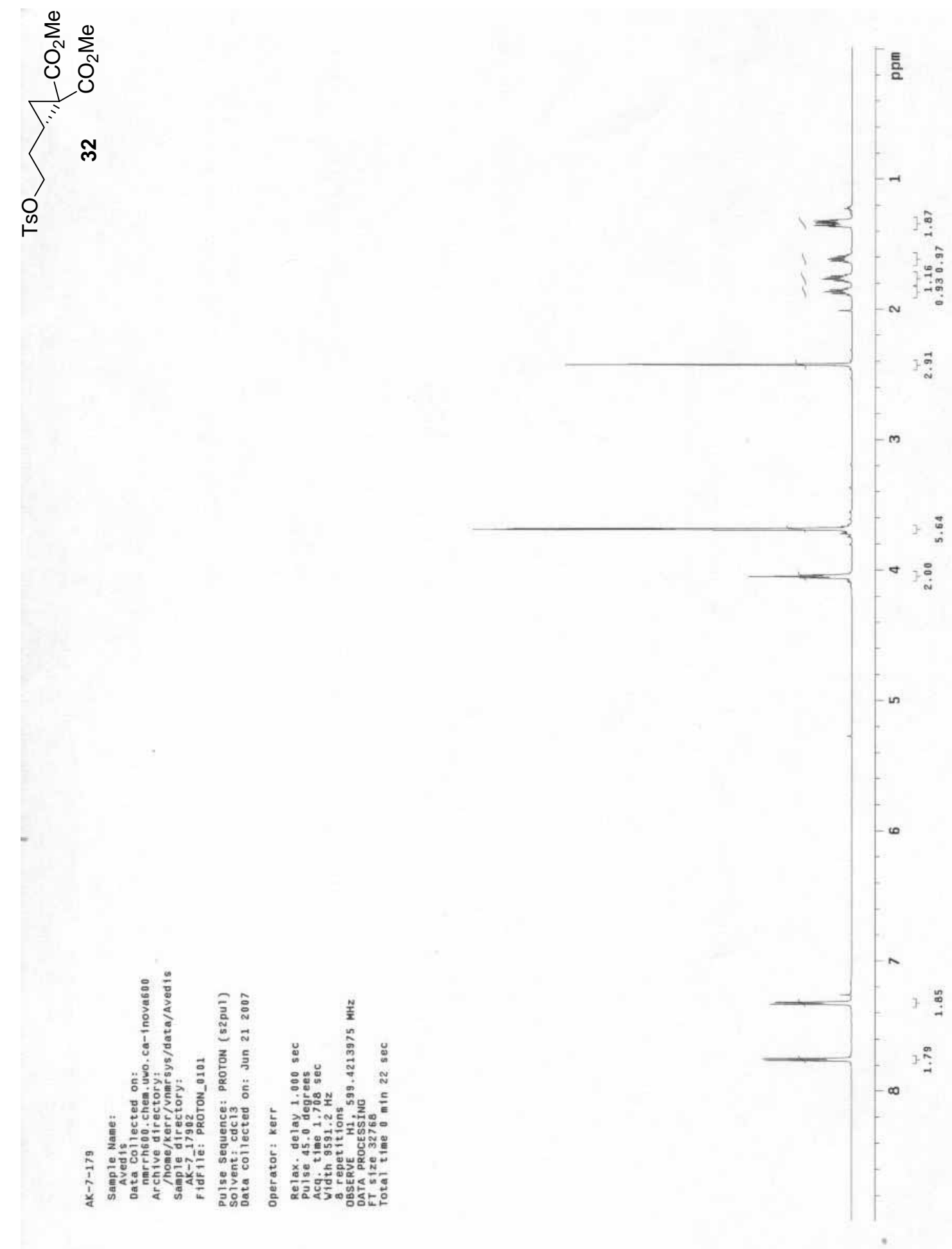
$\sum_{0}^{\infty} \infty$

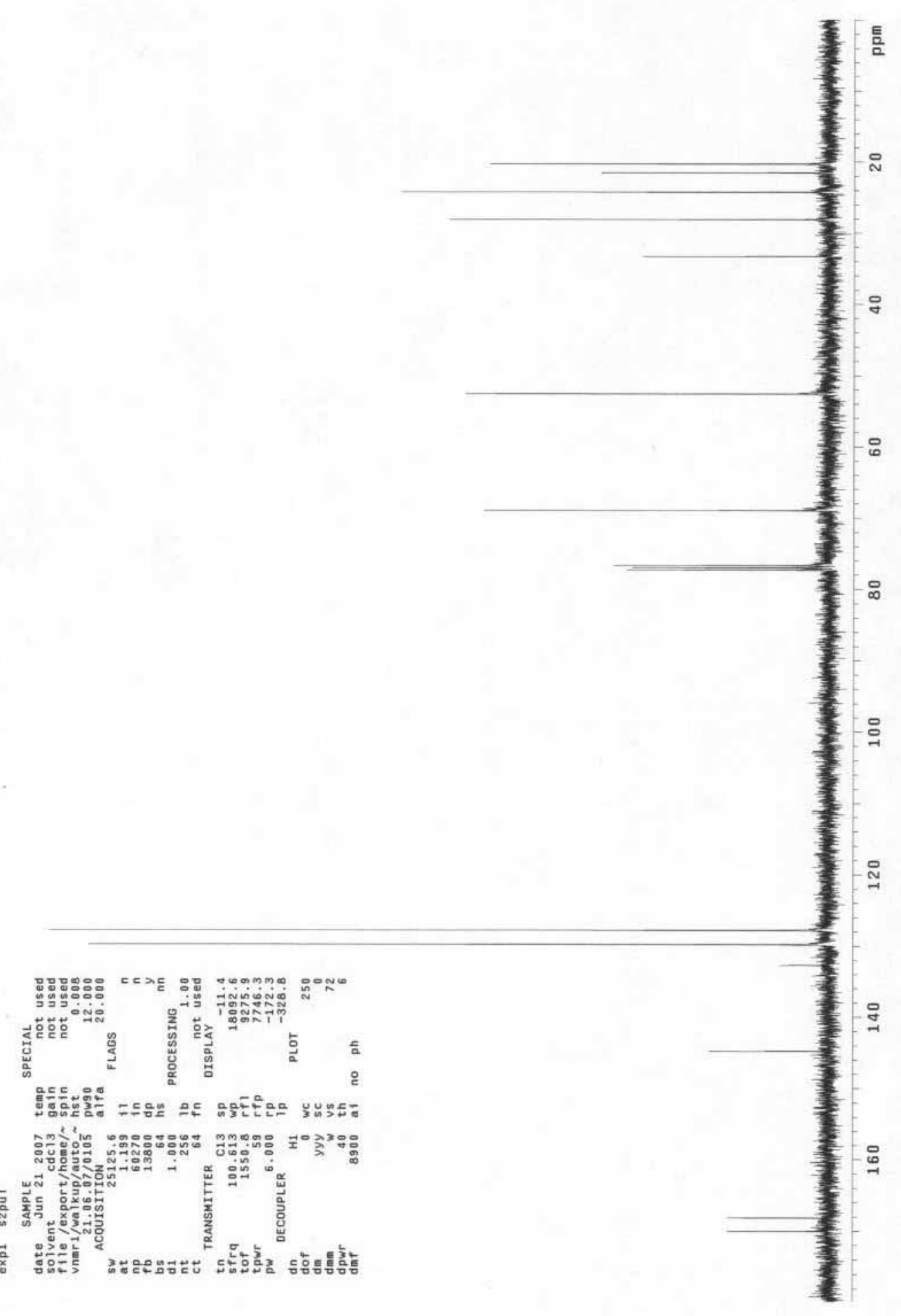




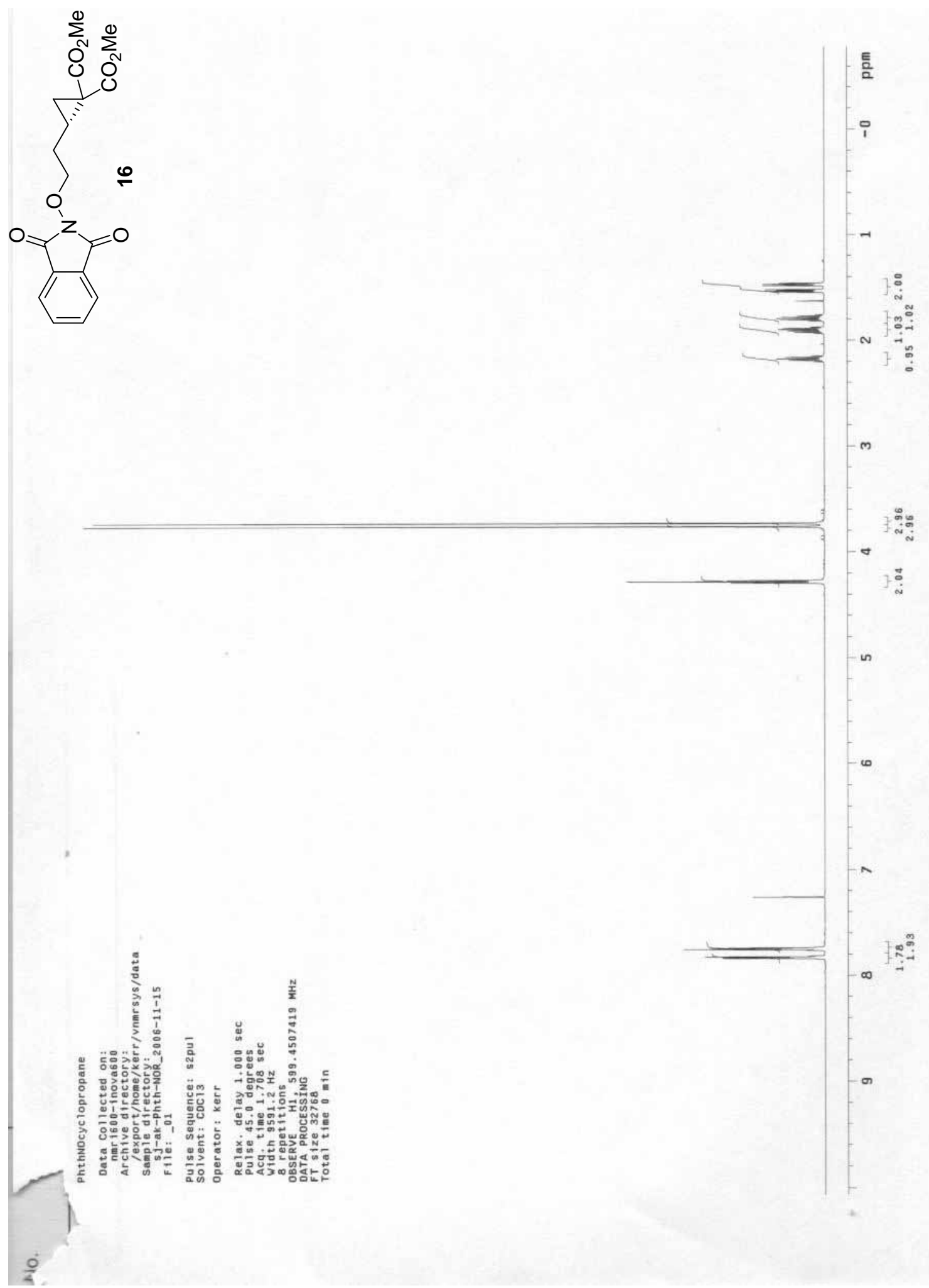



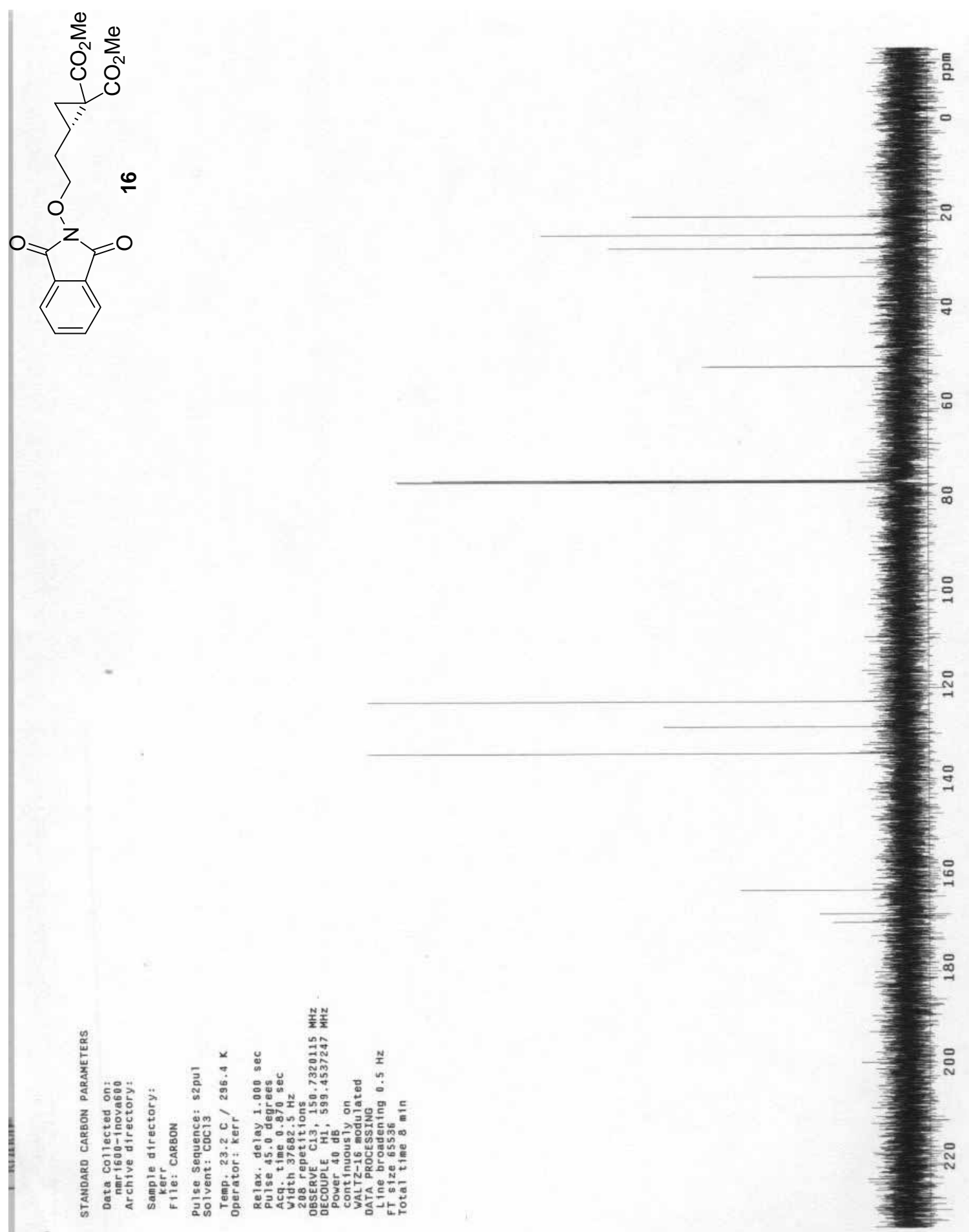


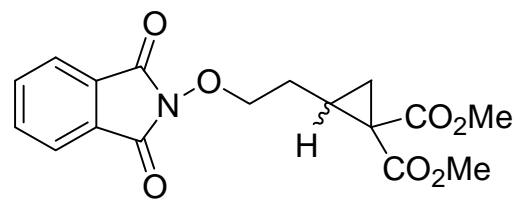

$(+l-)-16 ;(+)-\mathrm{Eu}(\mathrm{HfC})_{3}$

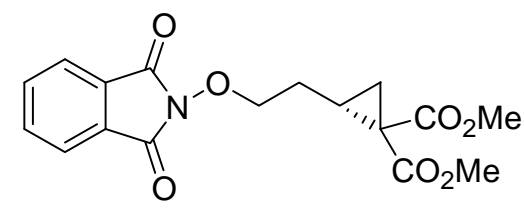

(S)-16; (+)-Eu(hfc) 3

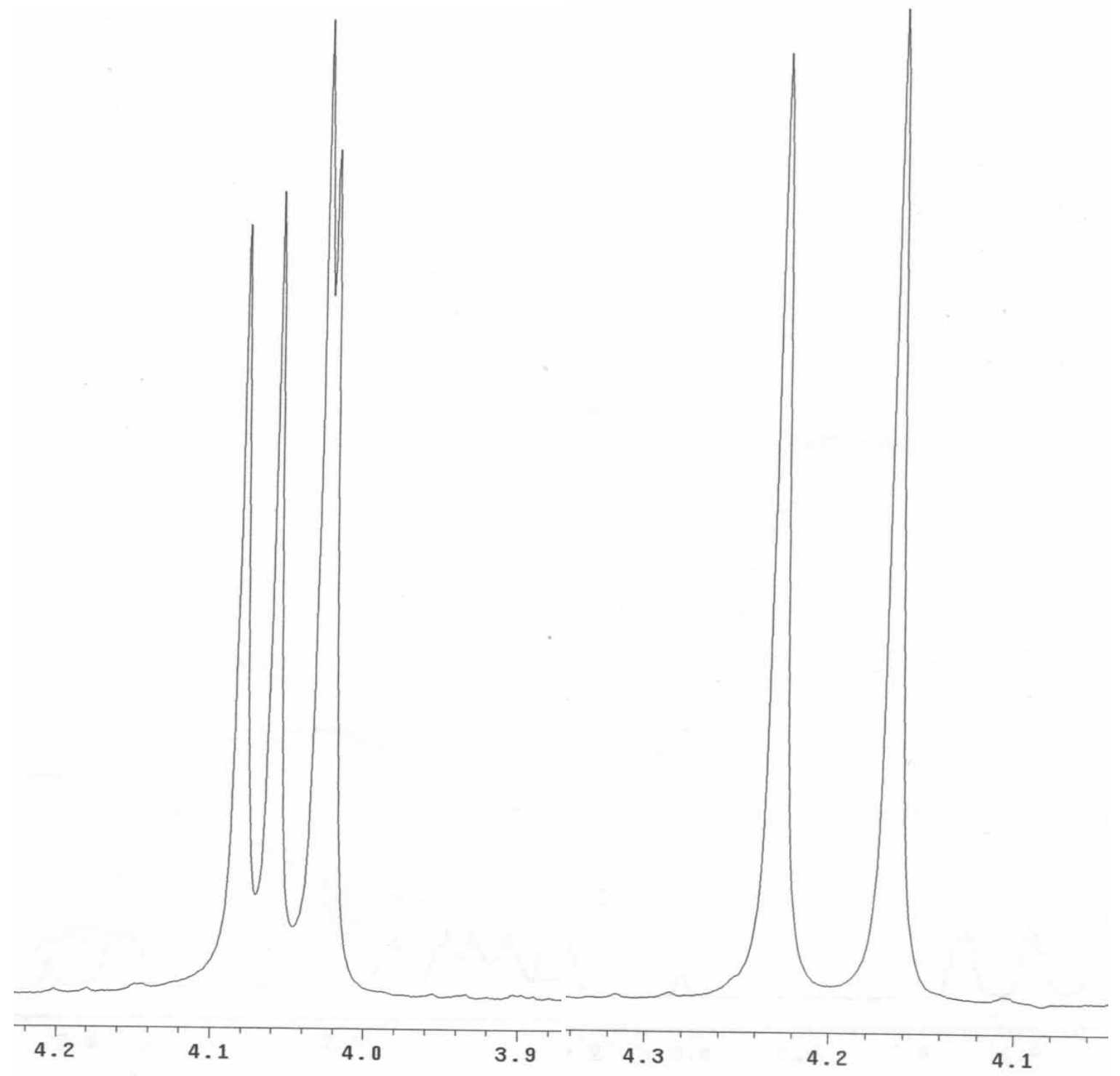



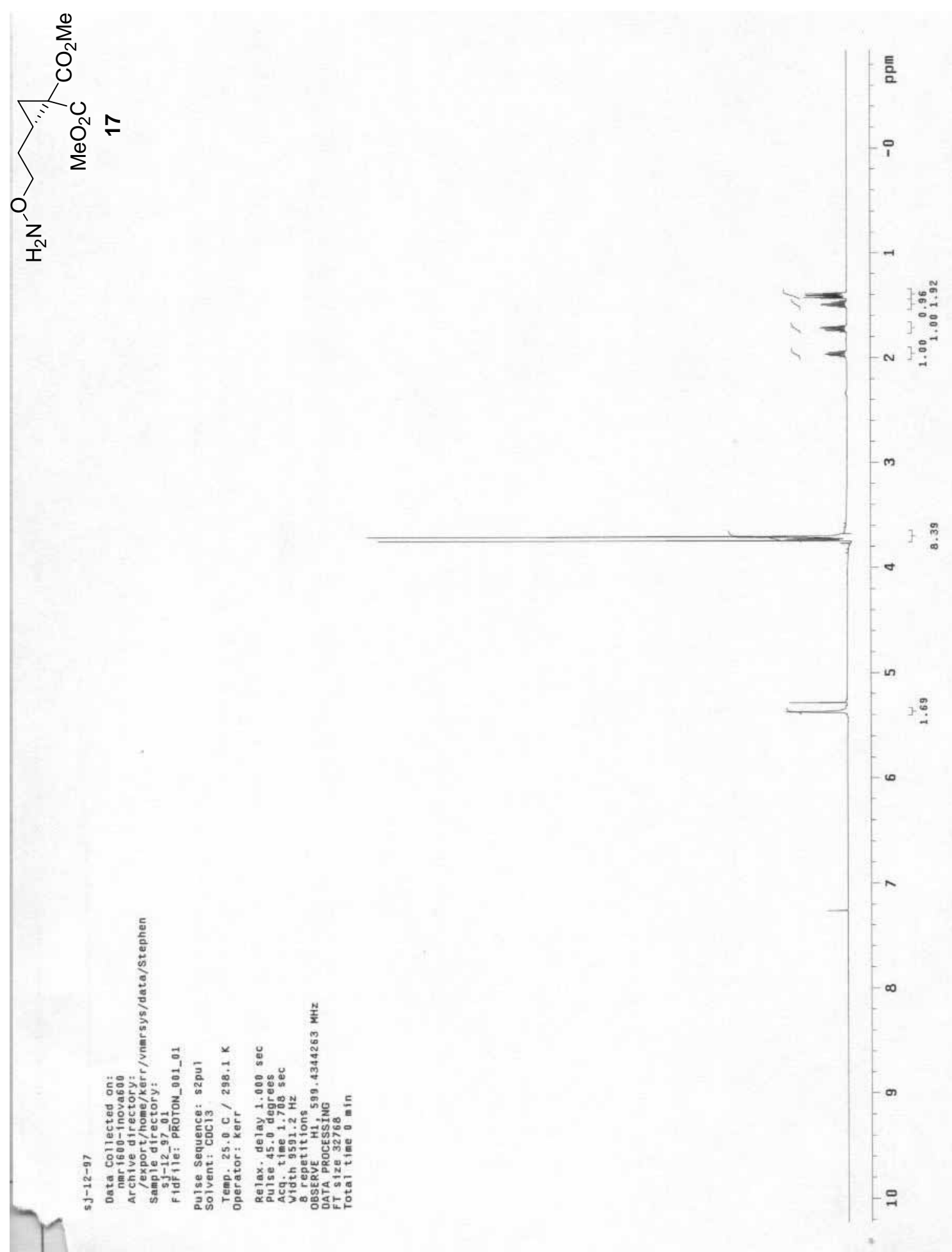

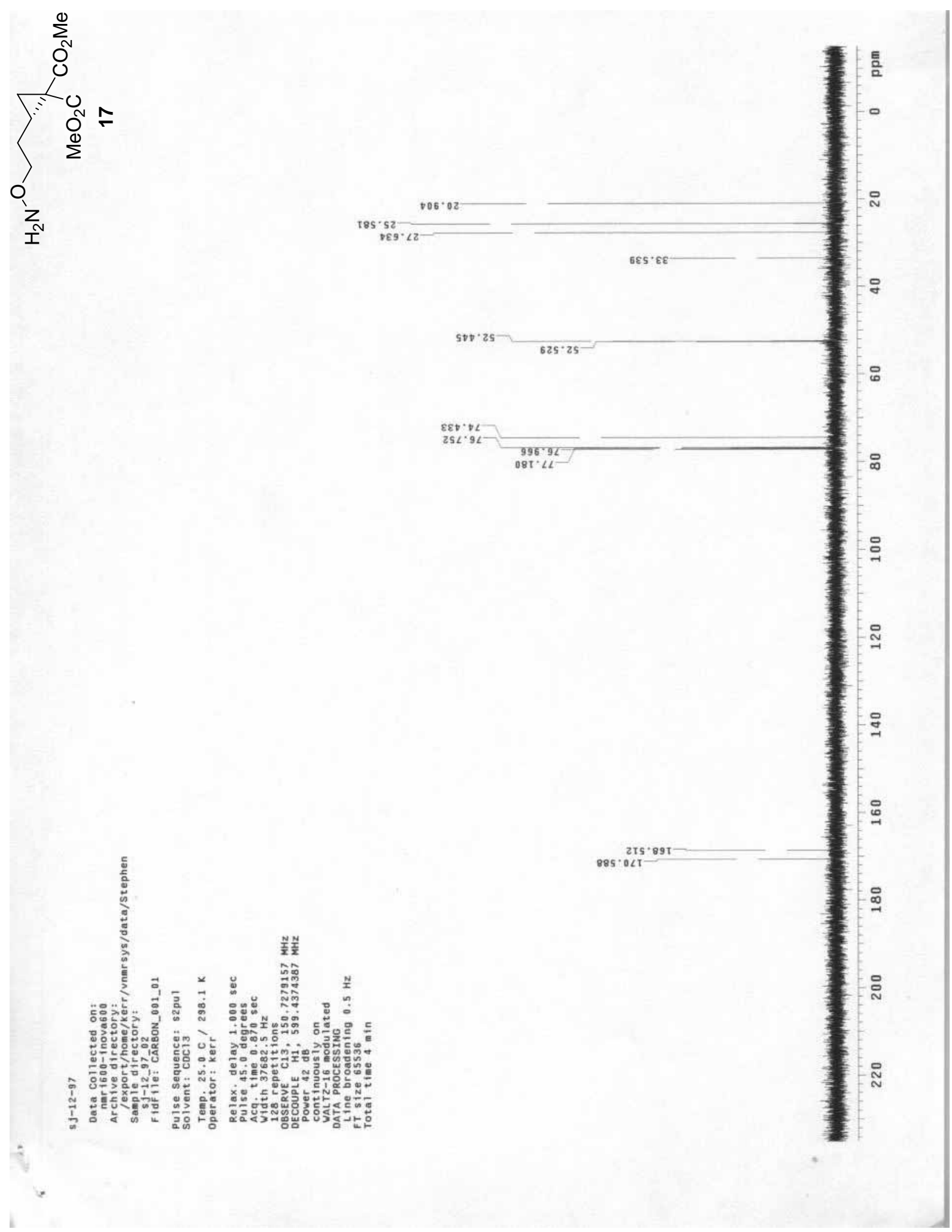


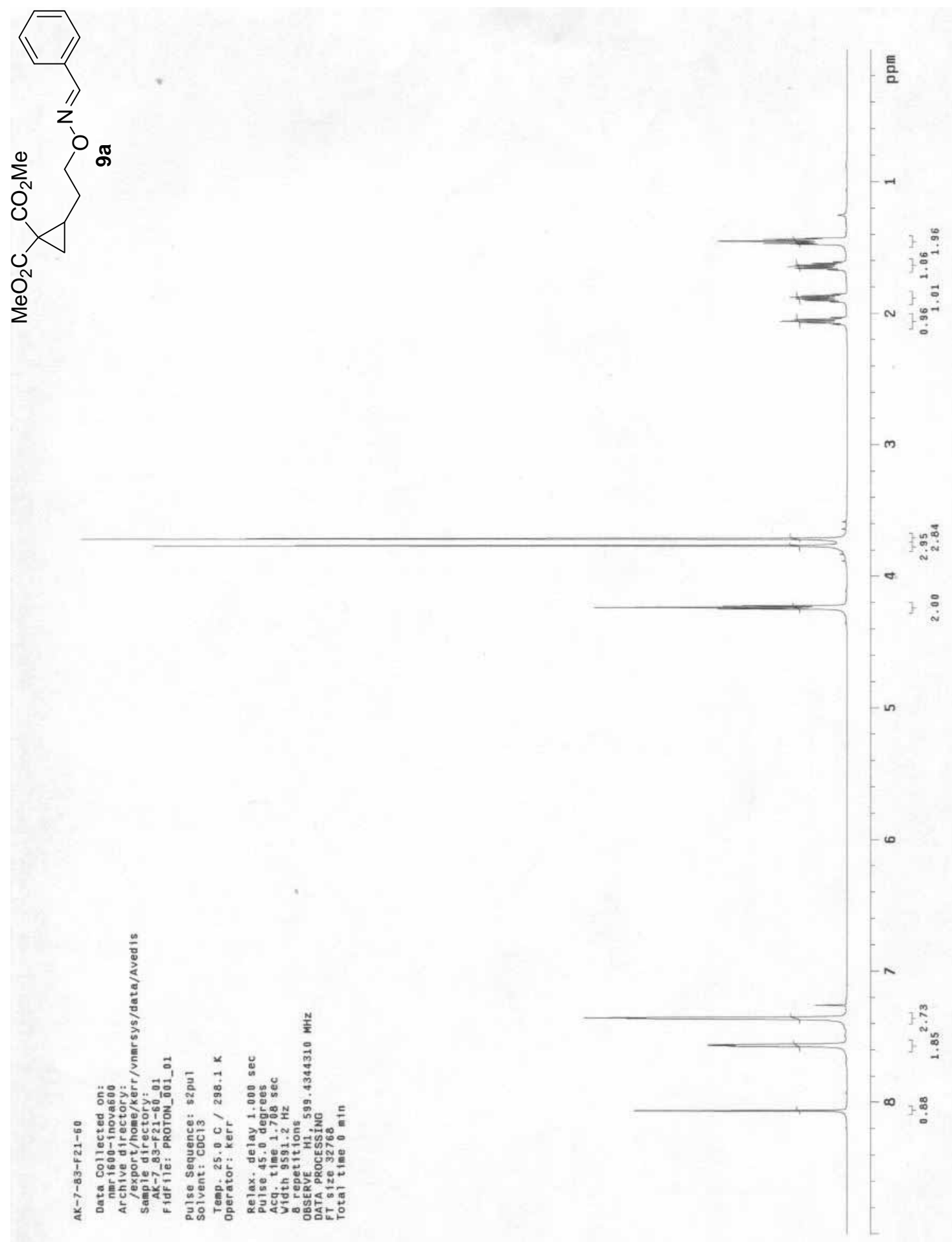



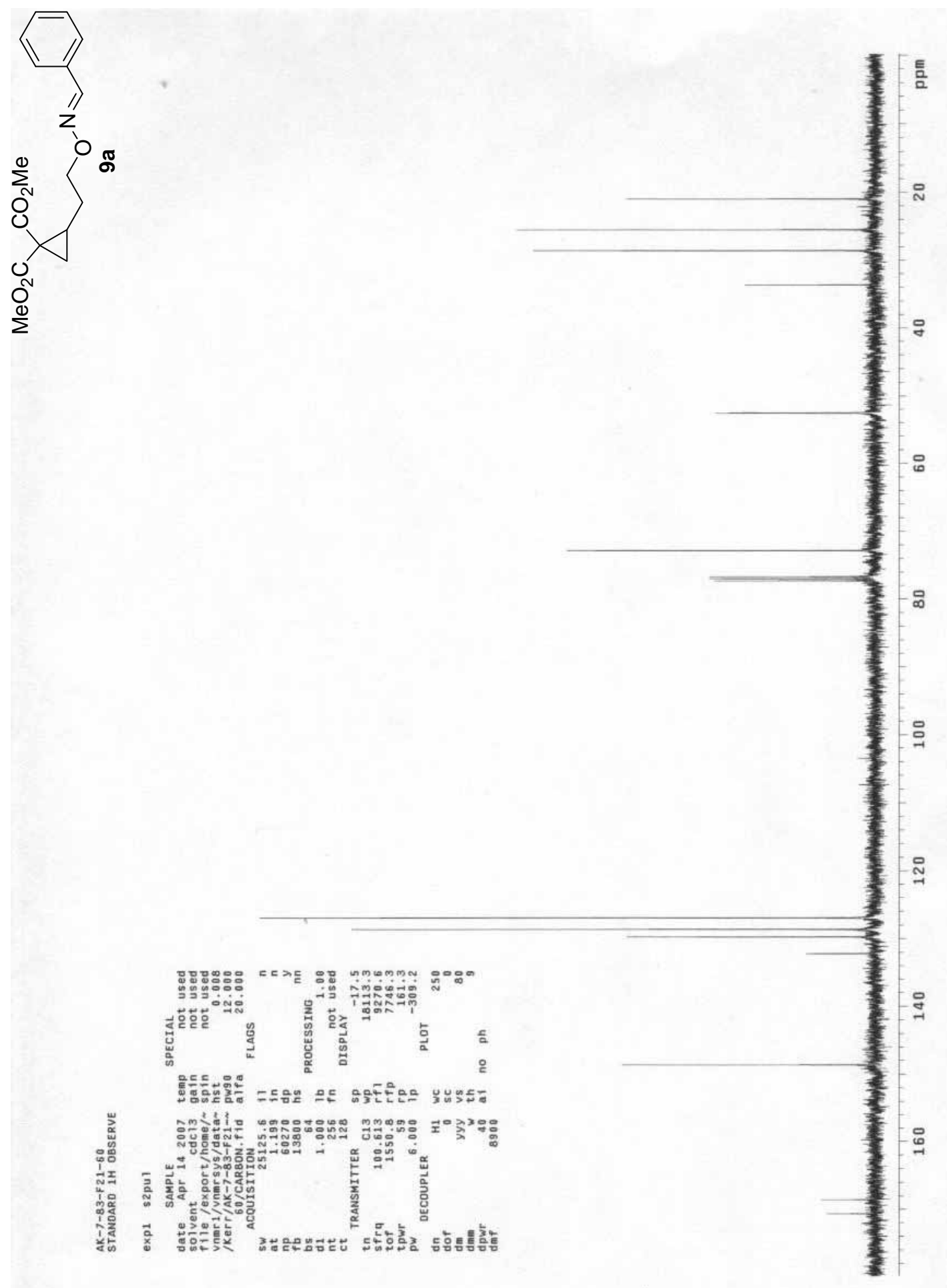


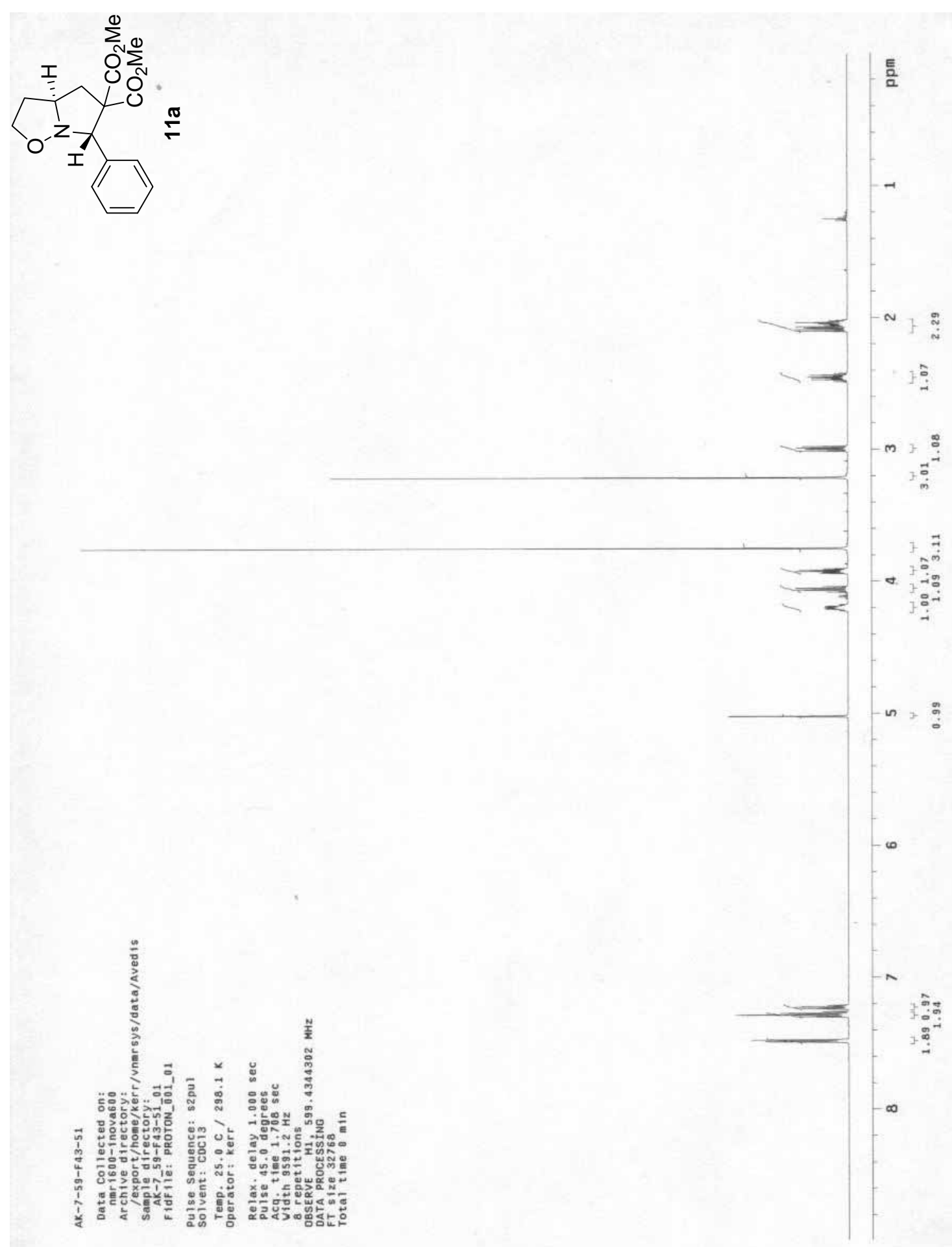



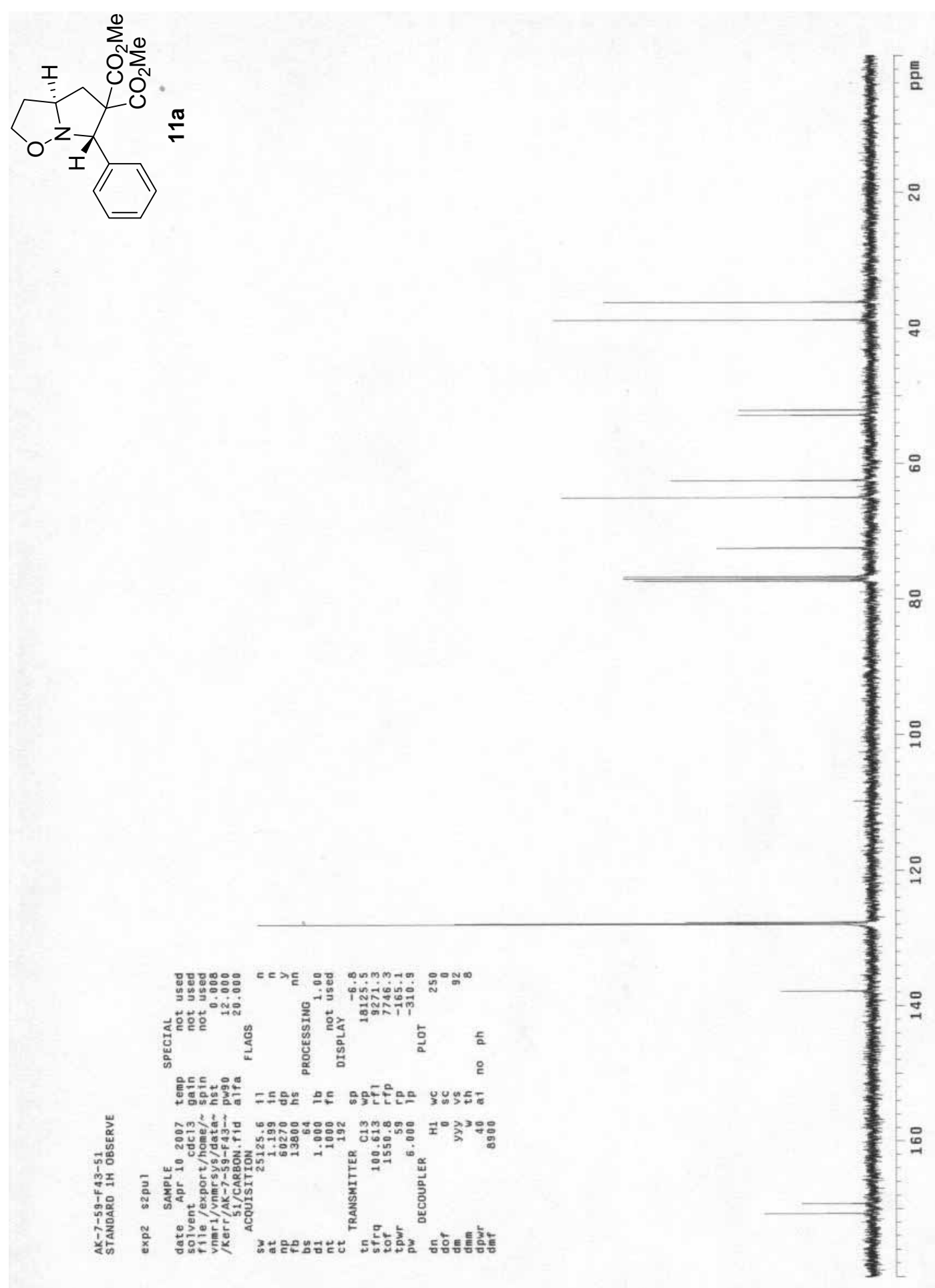


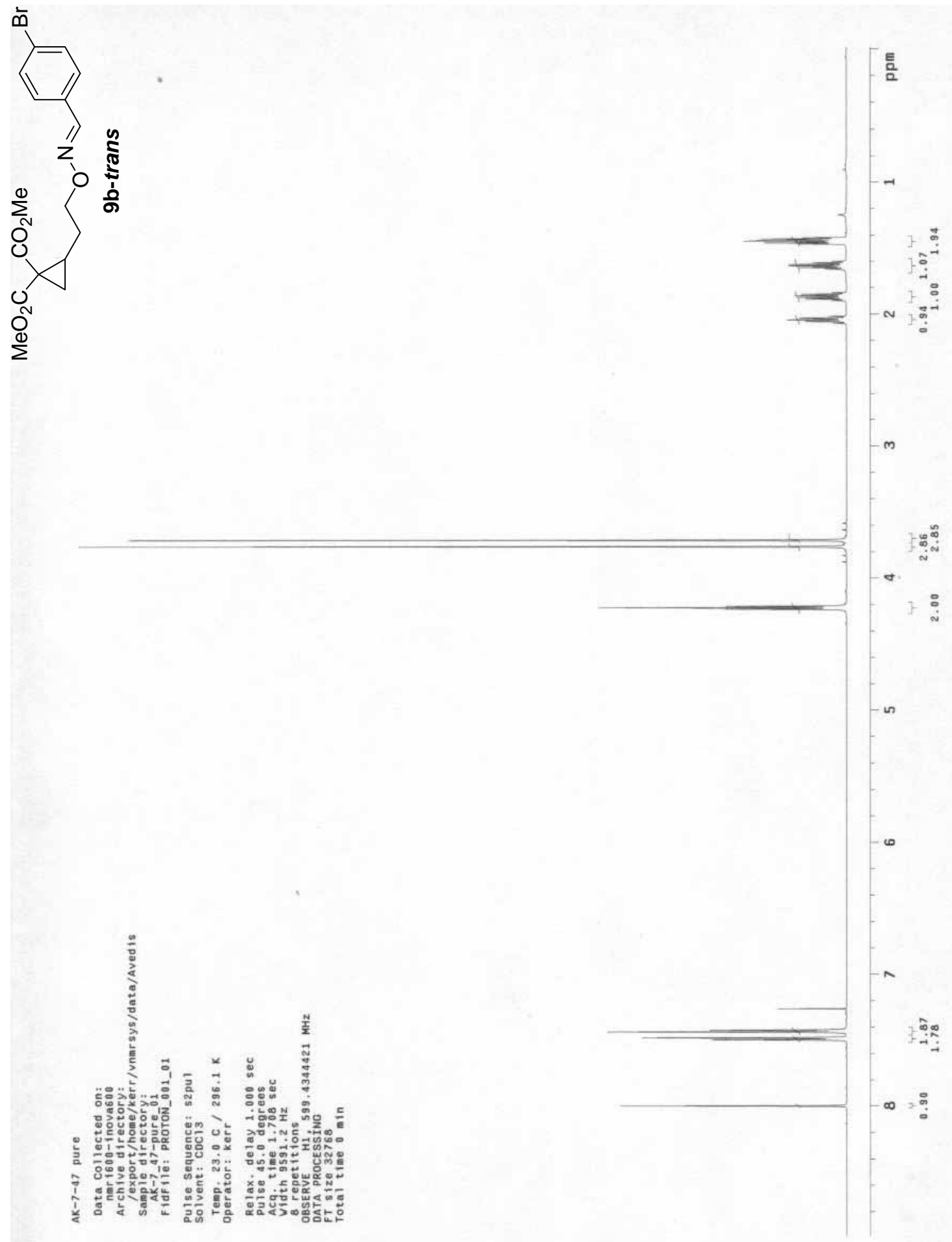




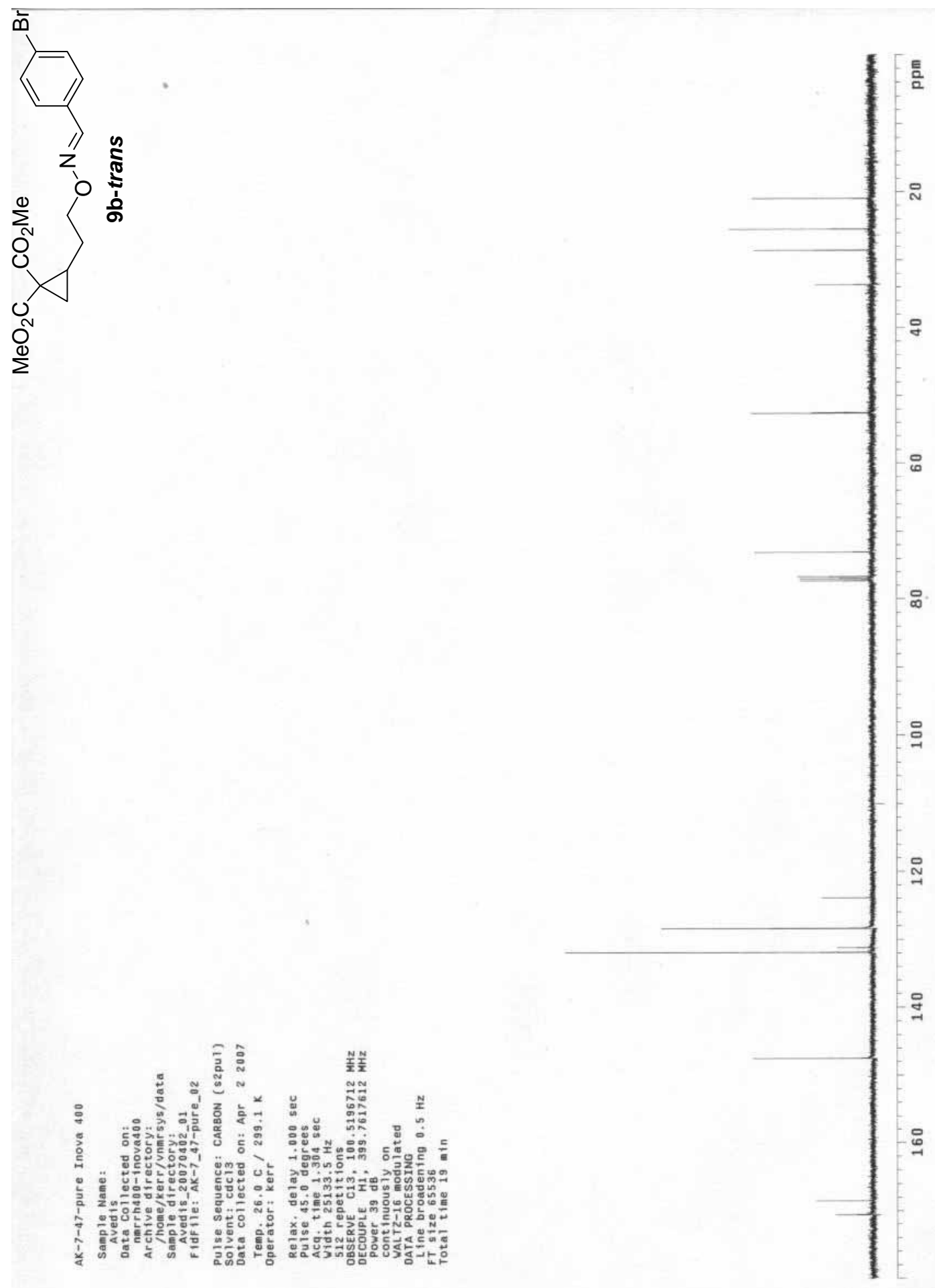



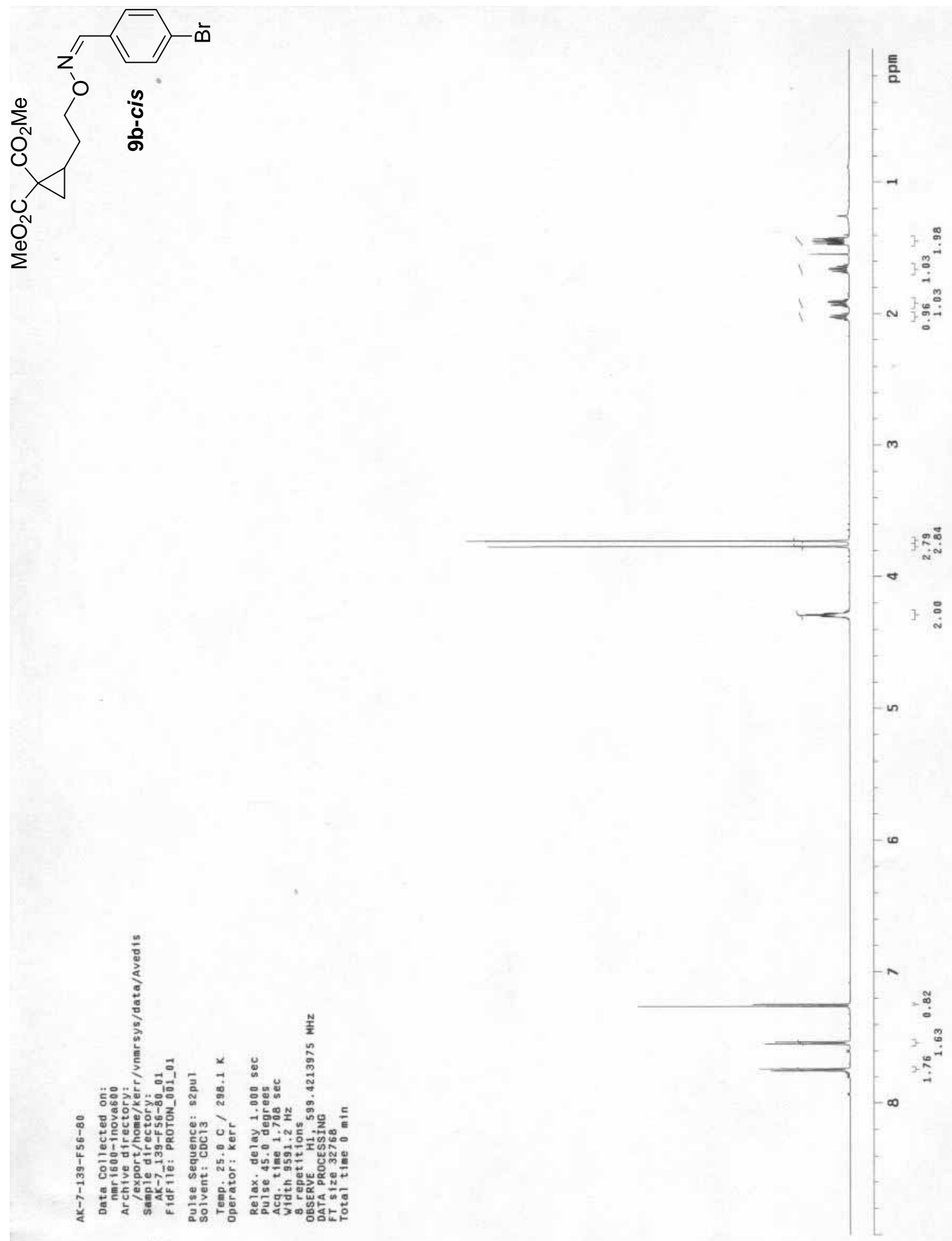

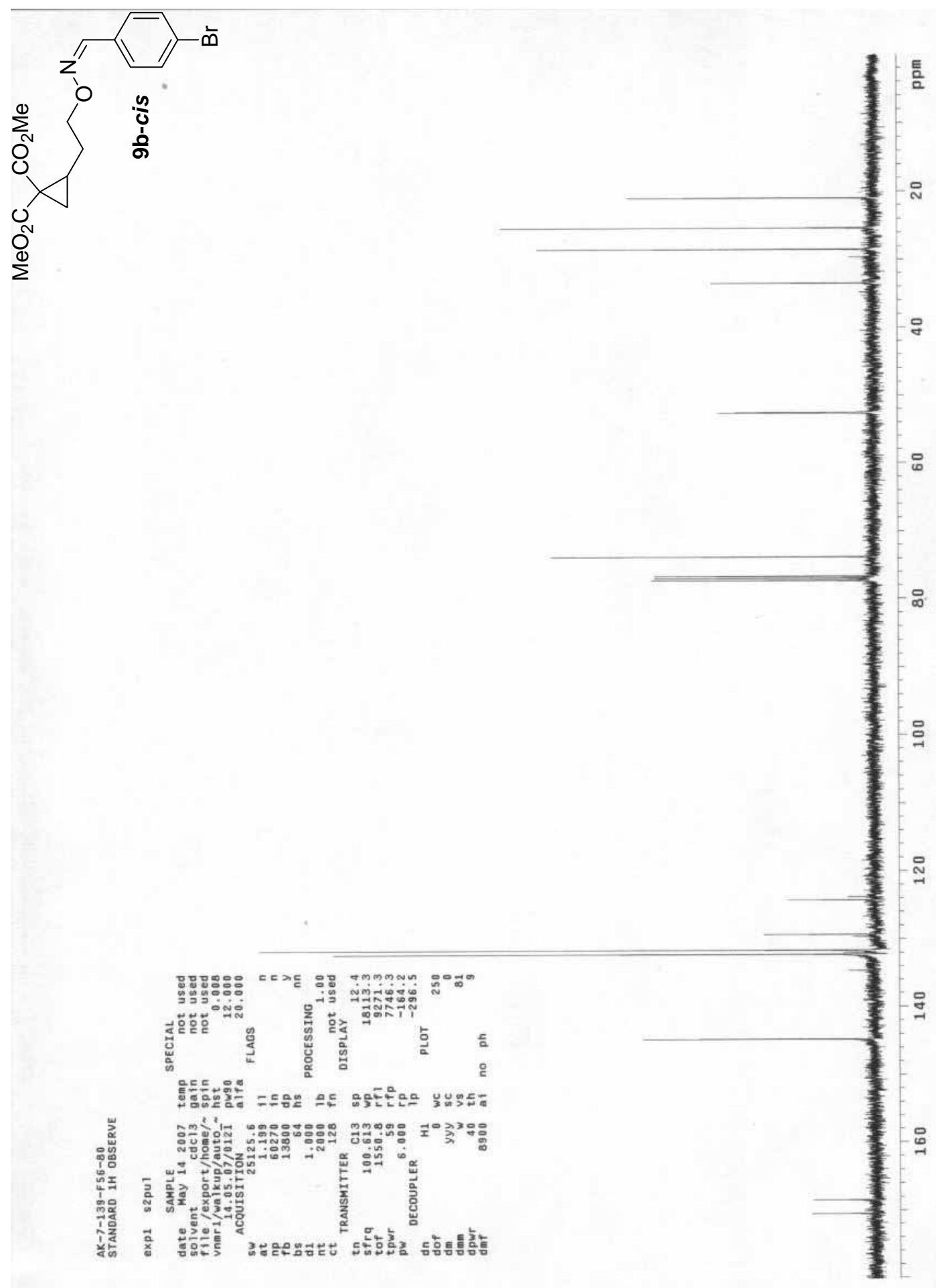


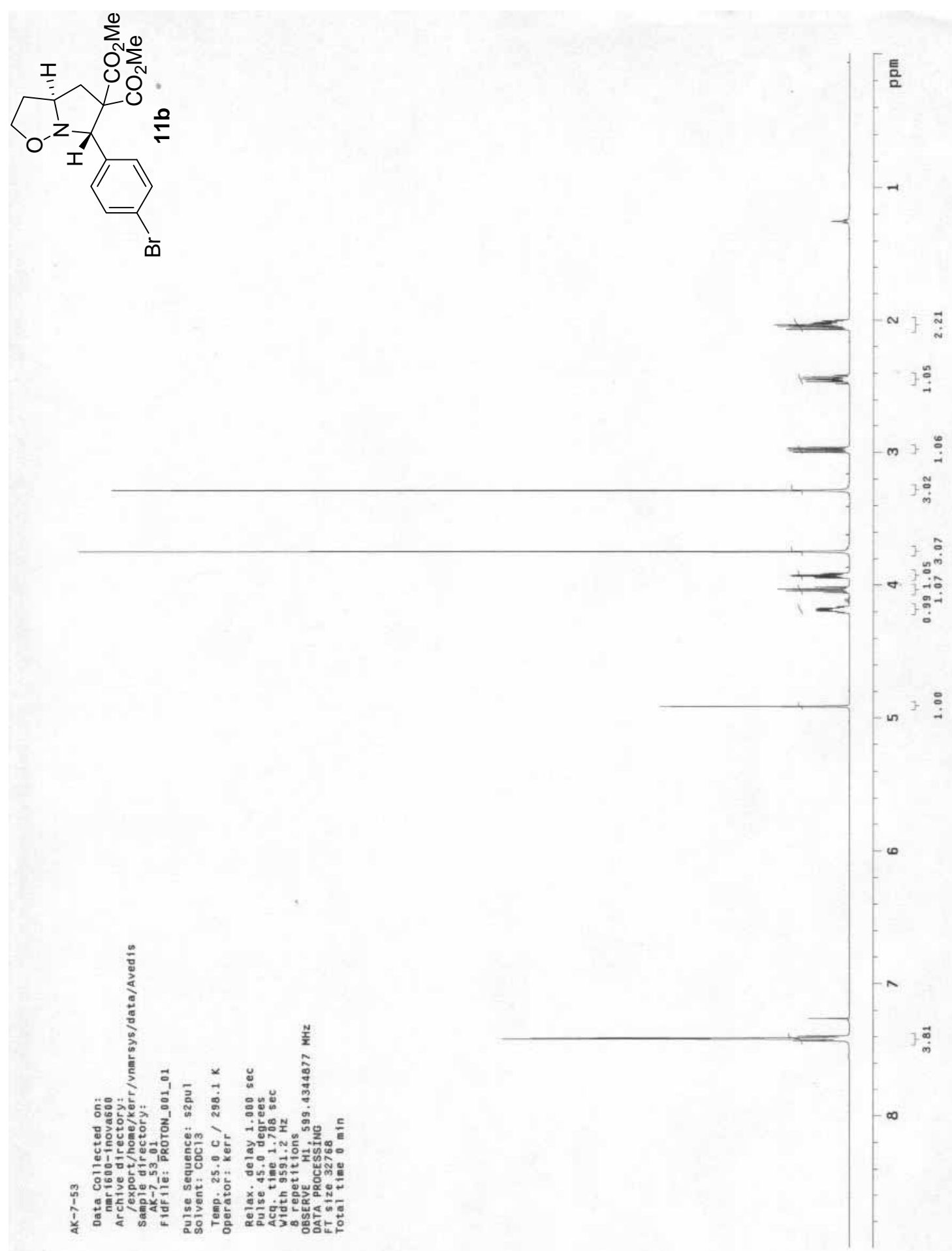



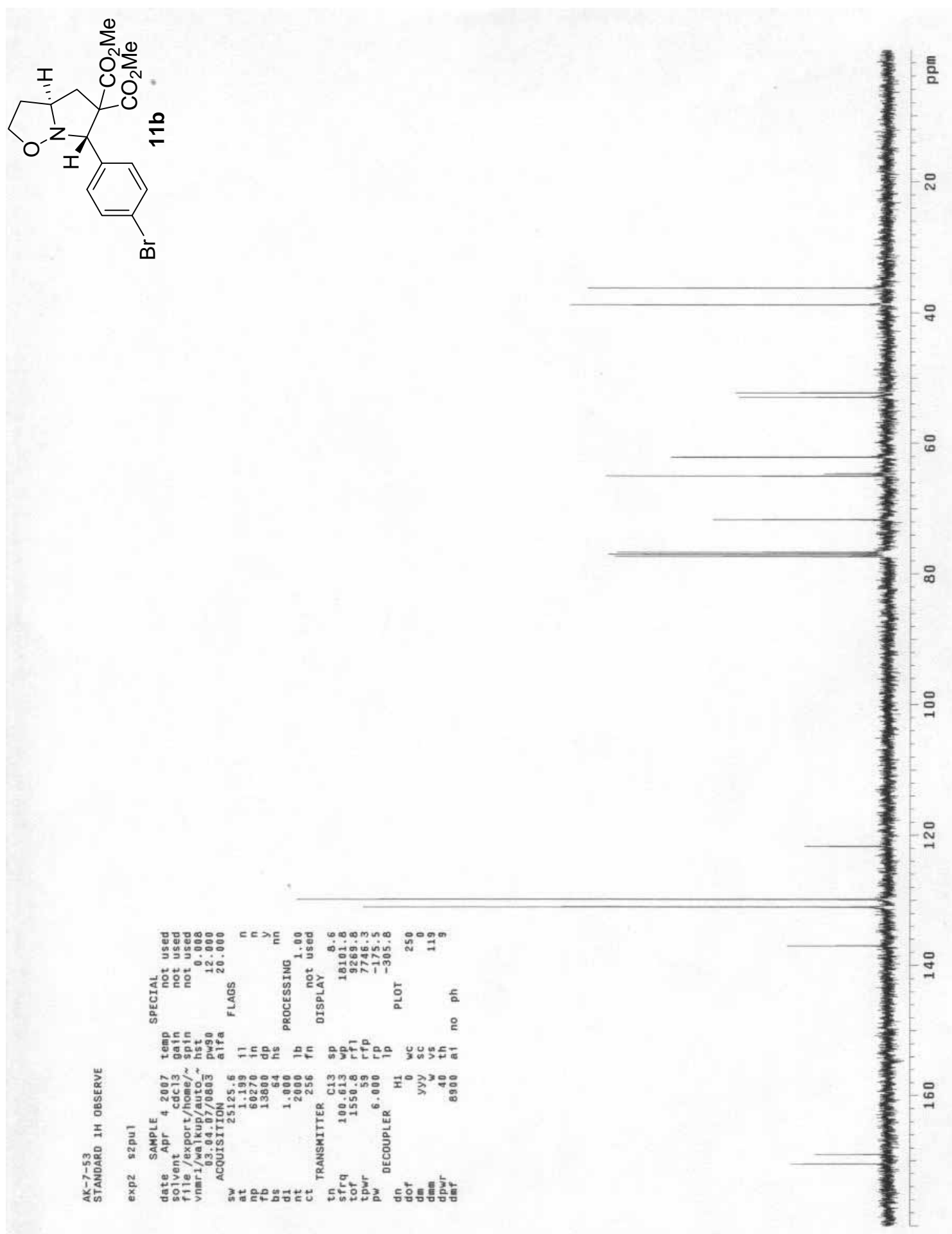


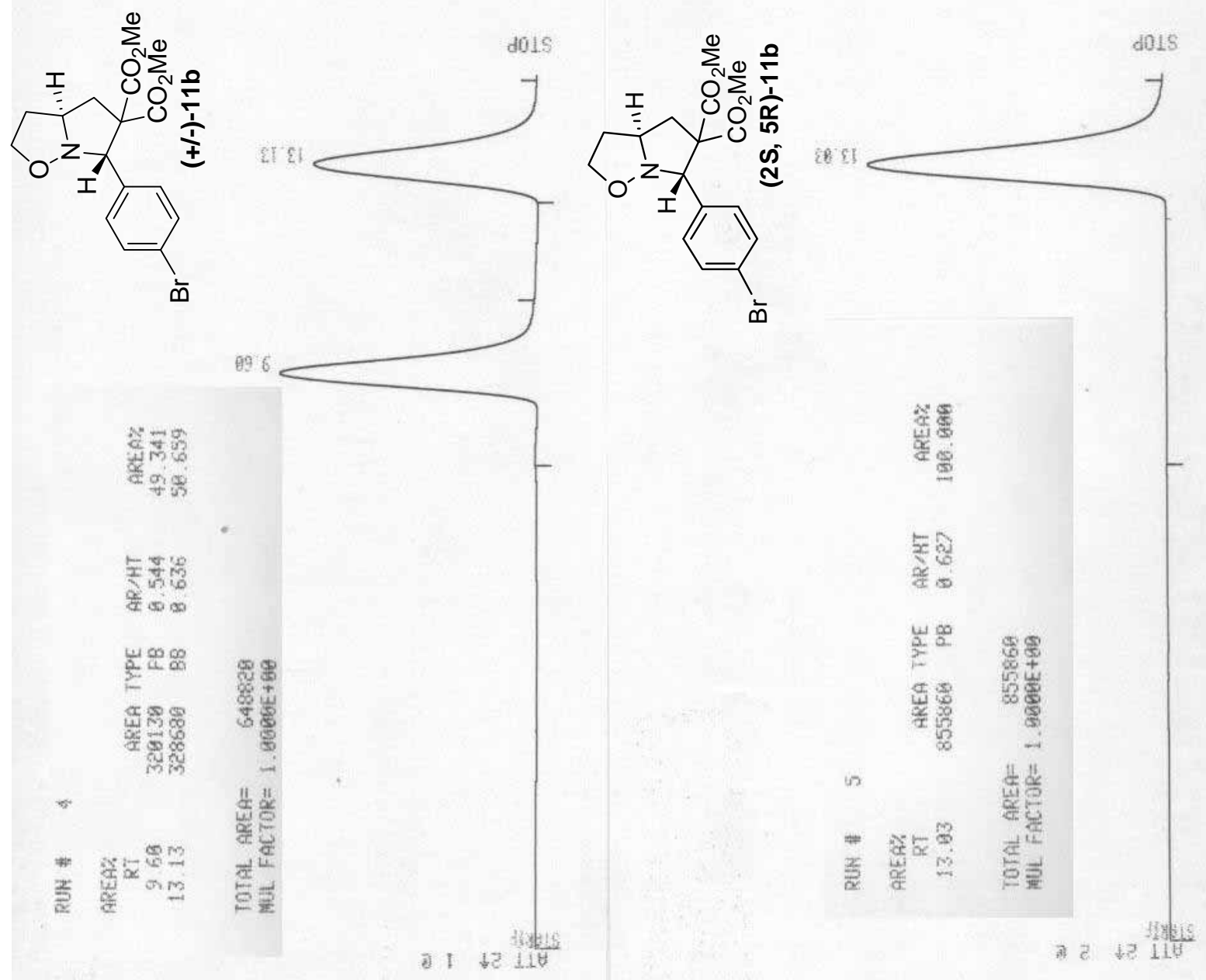




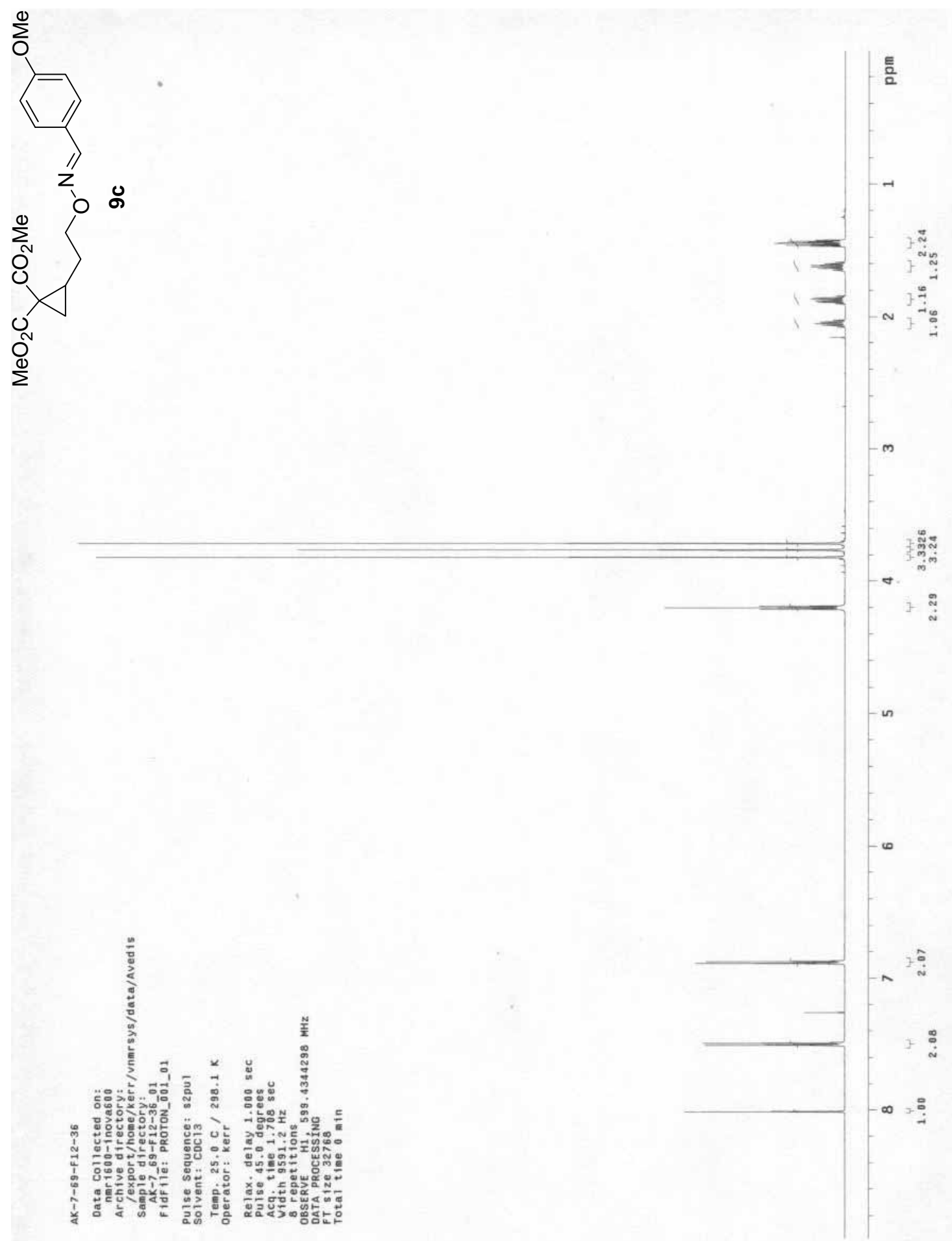




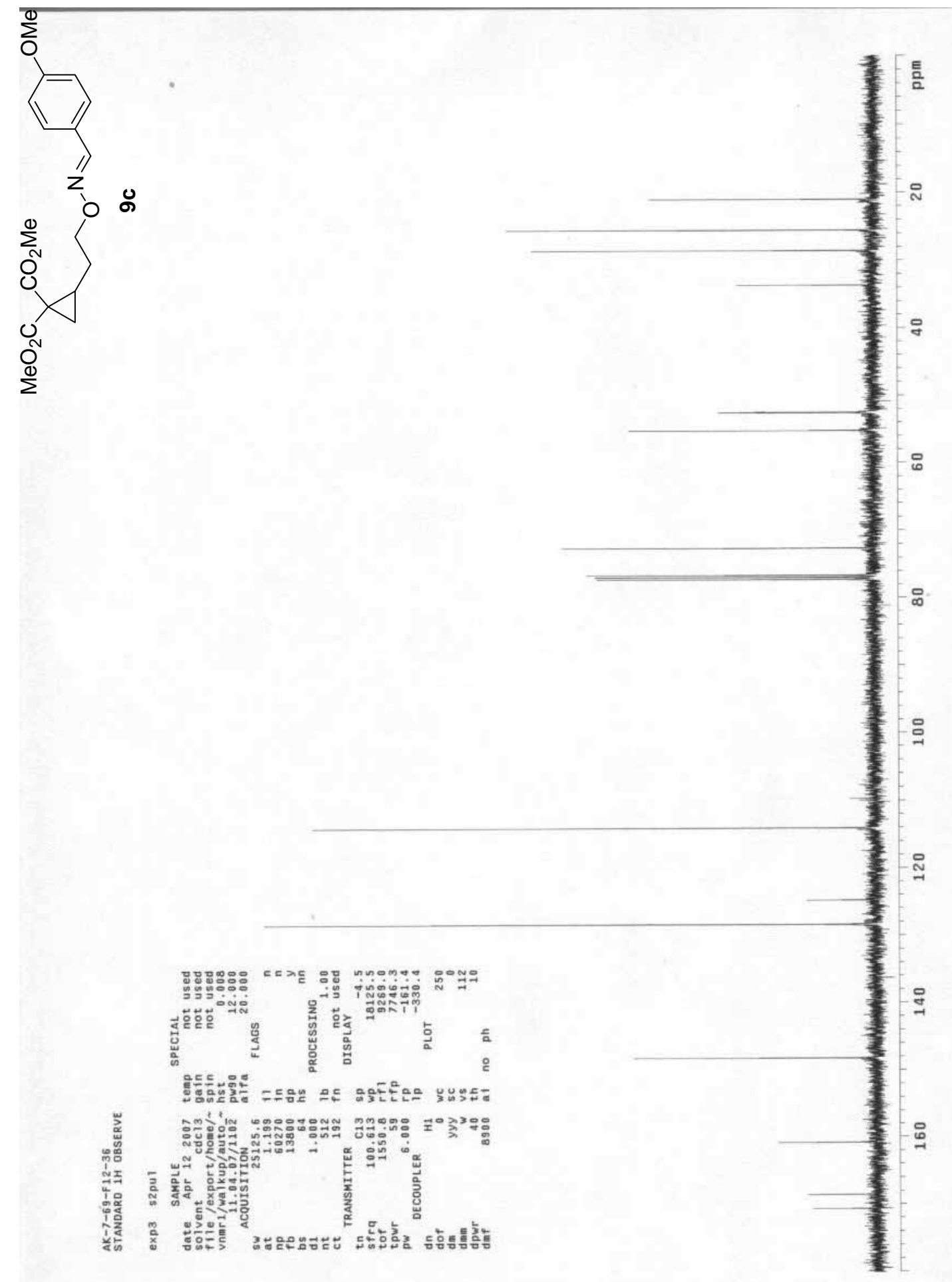




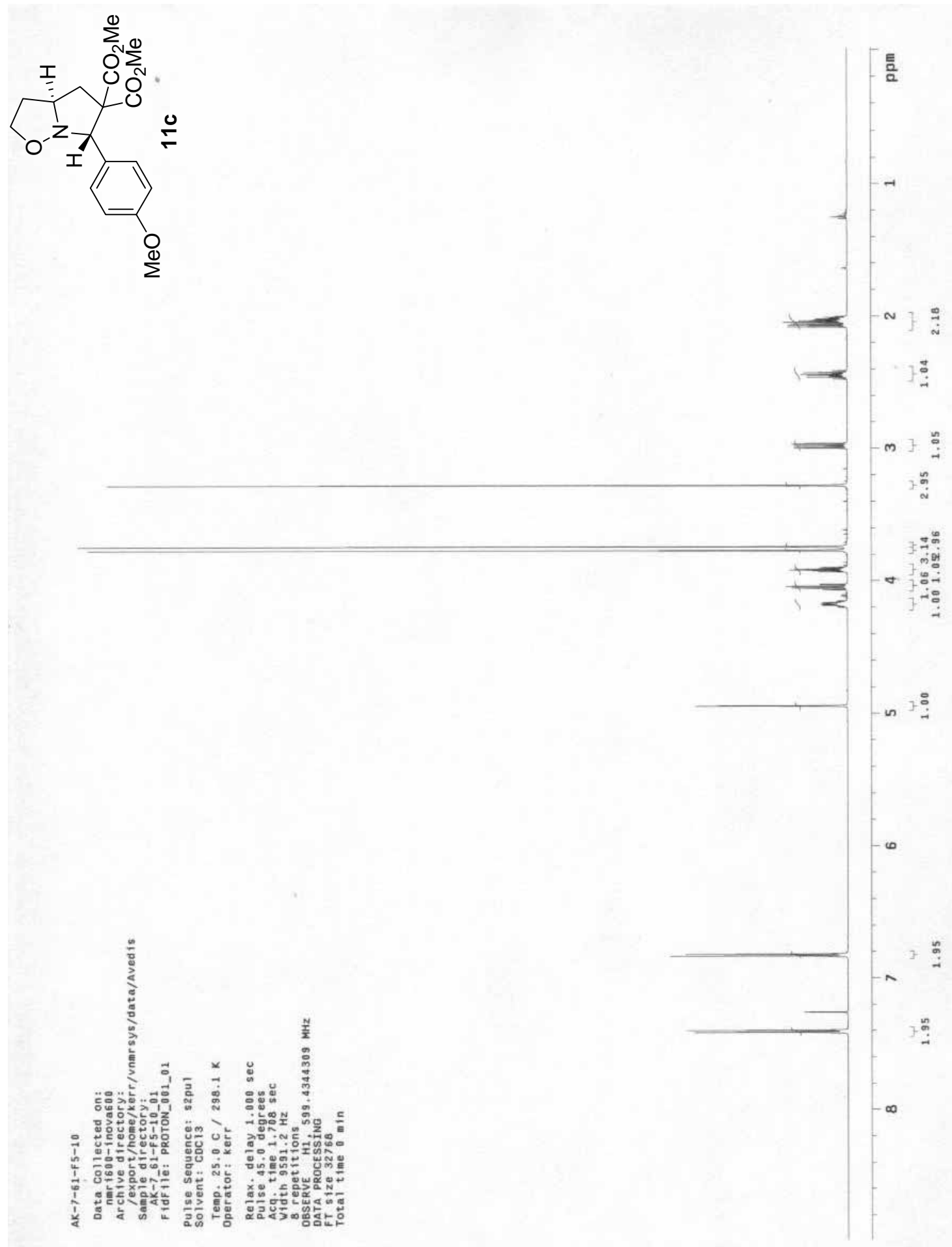



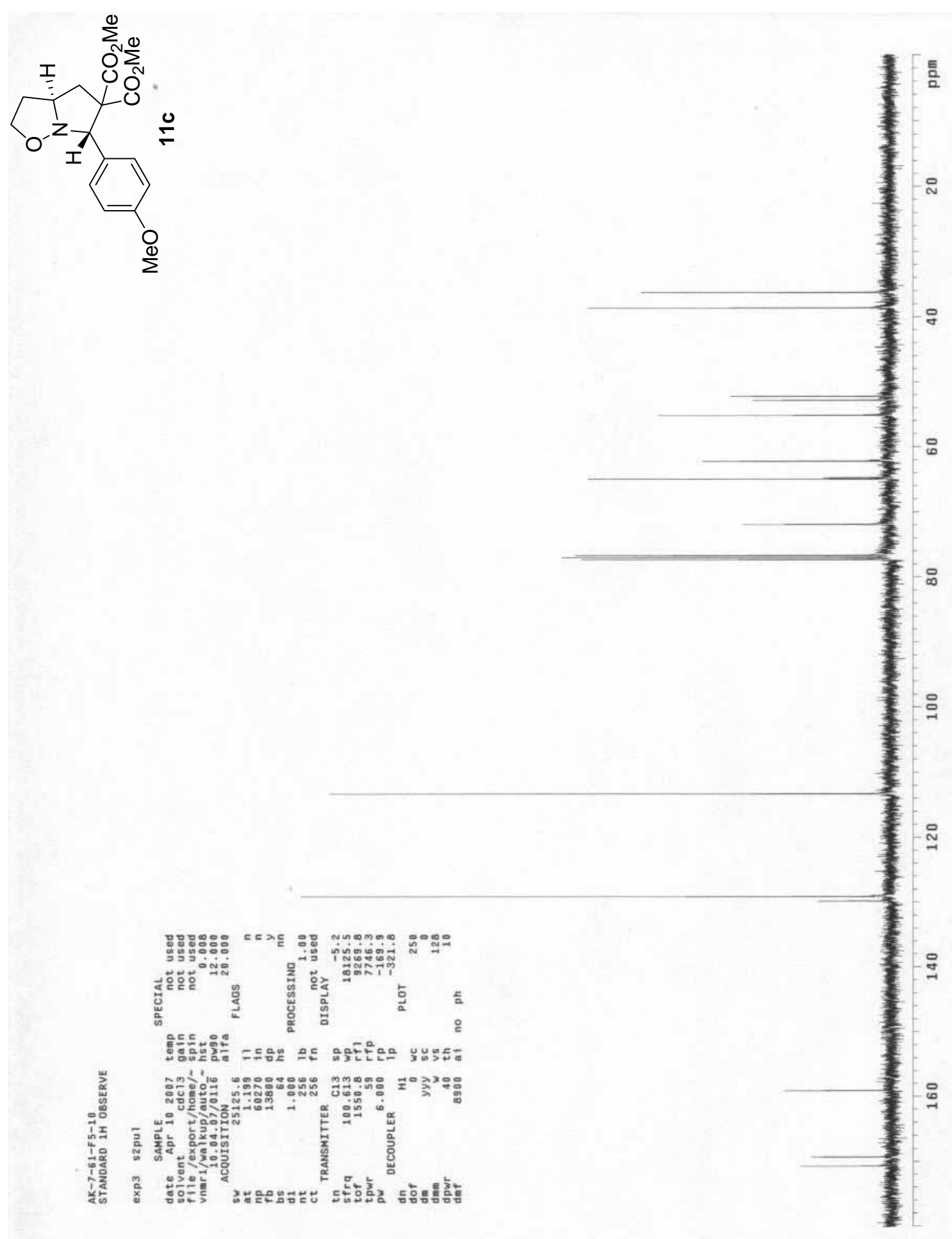


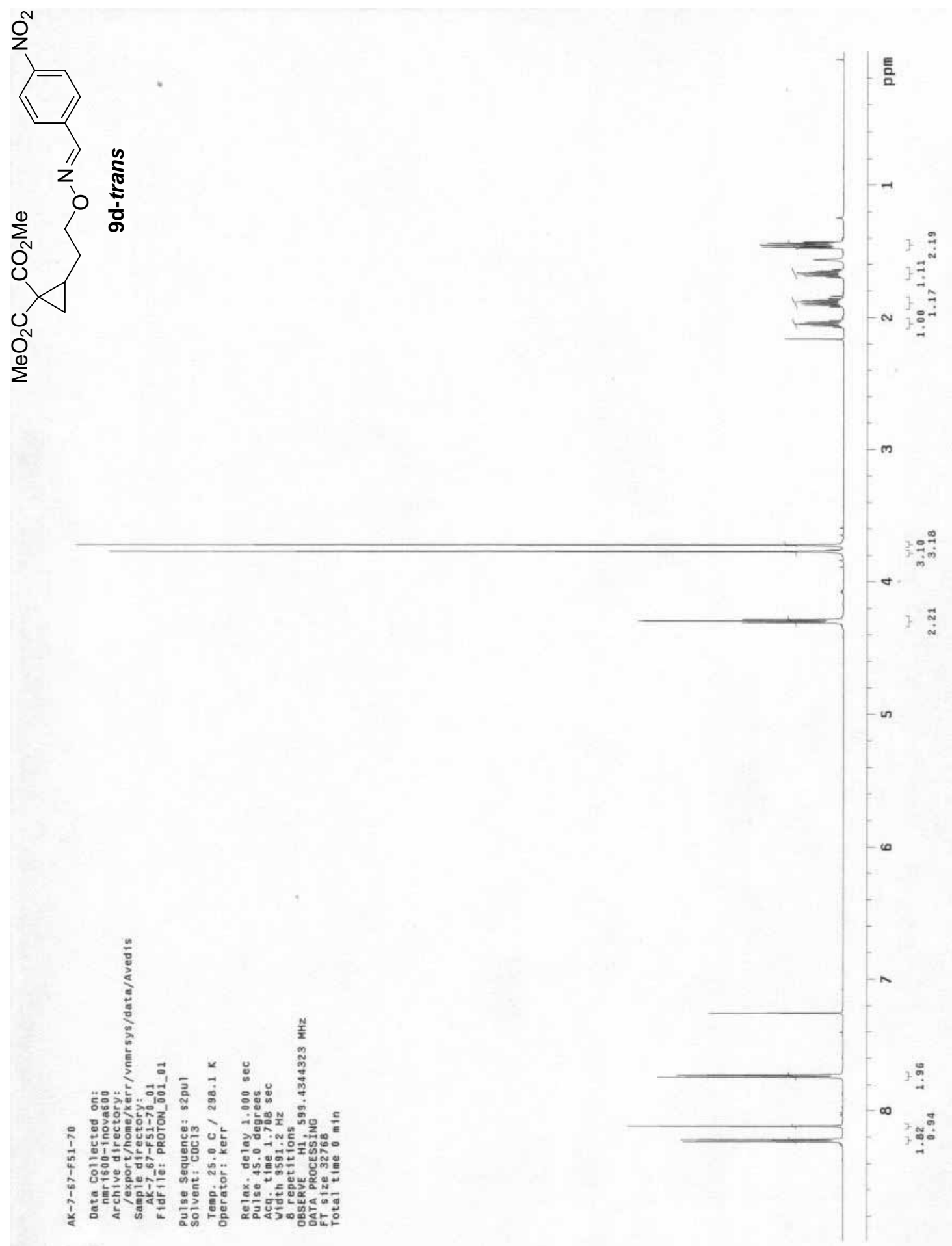




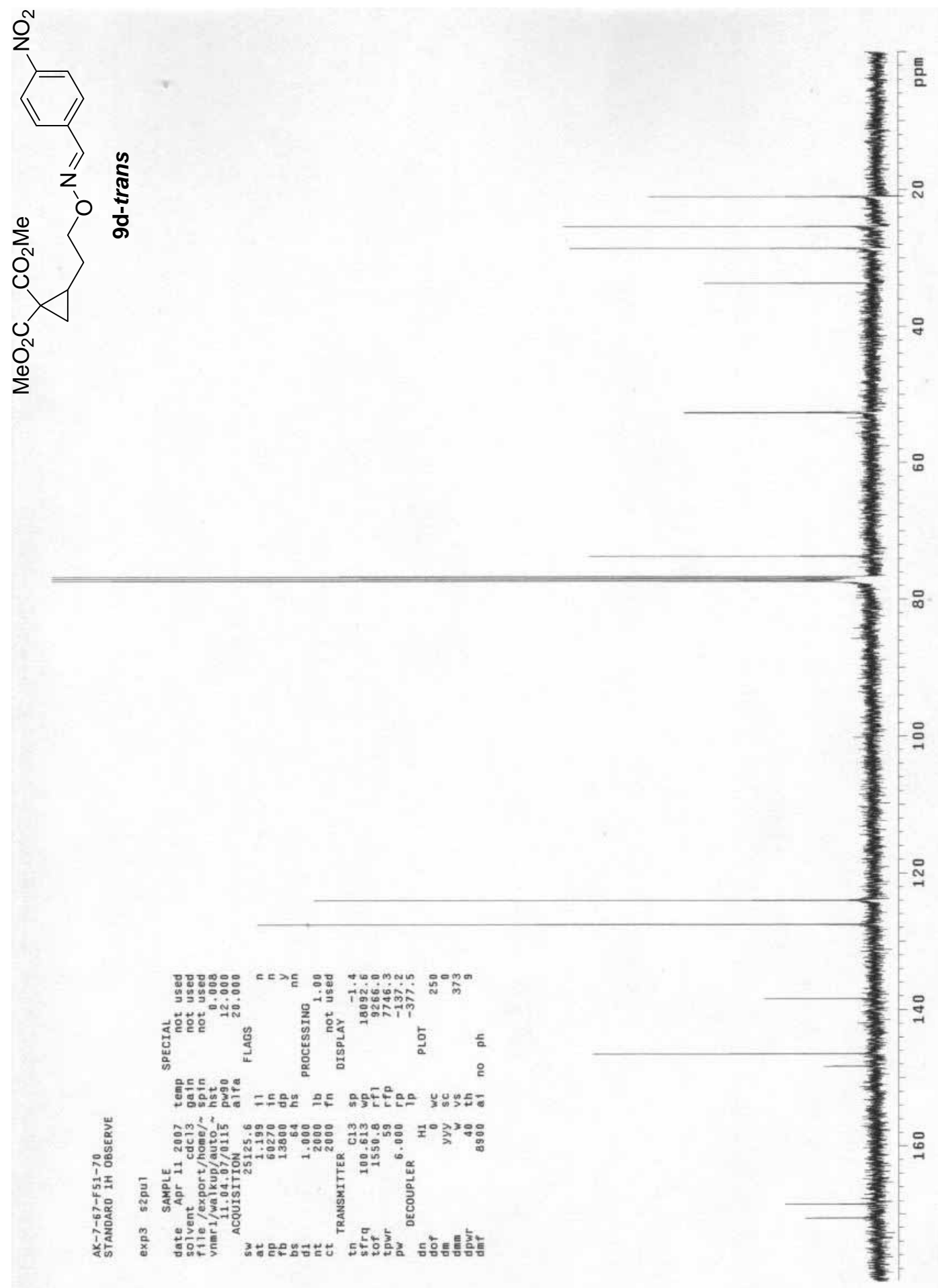



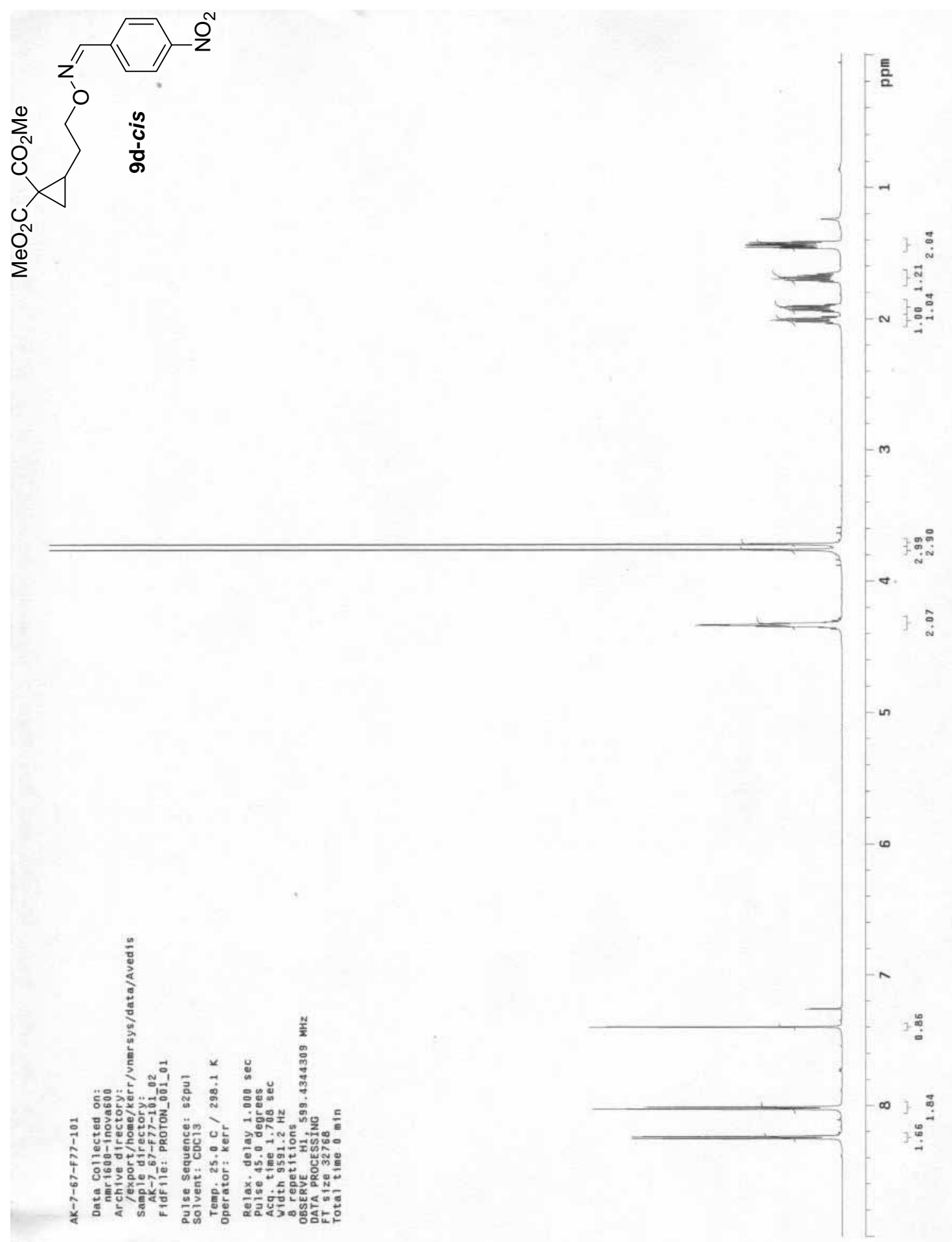

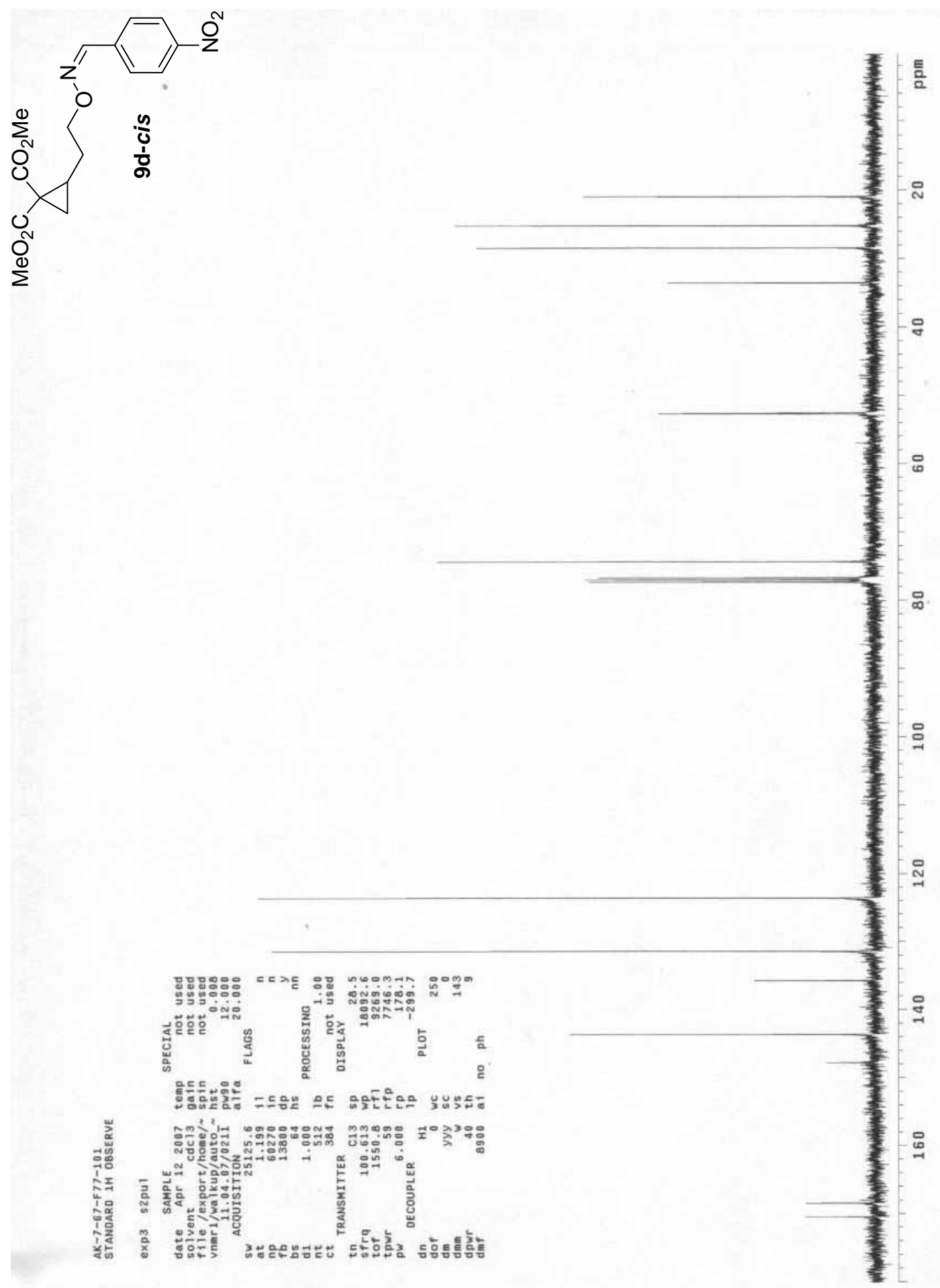

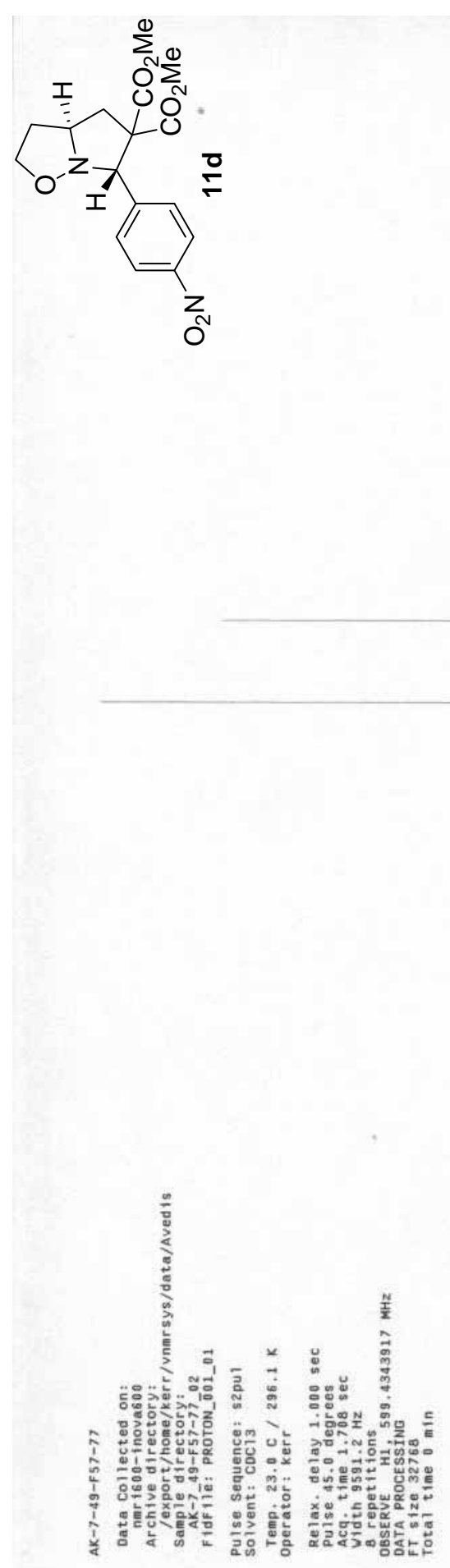

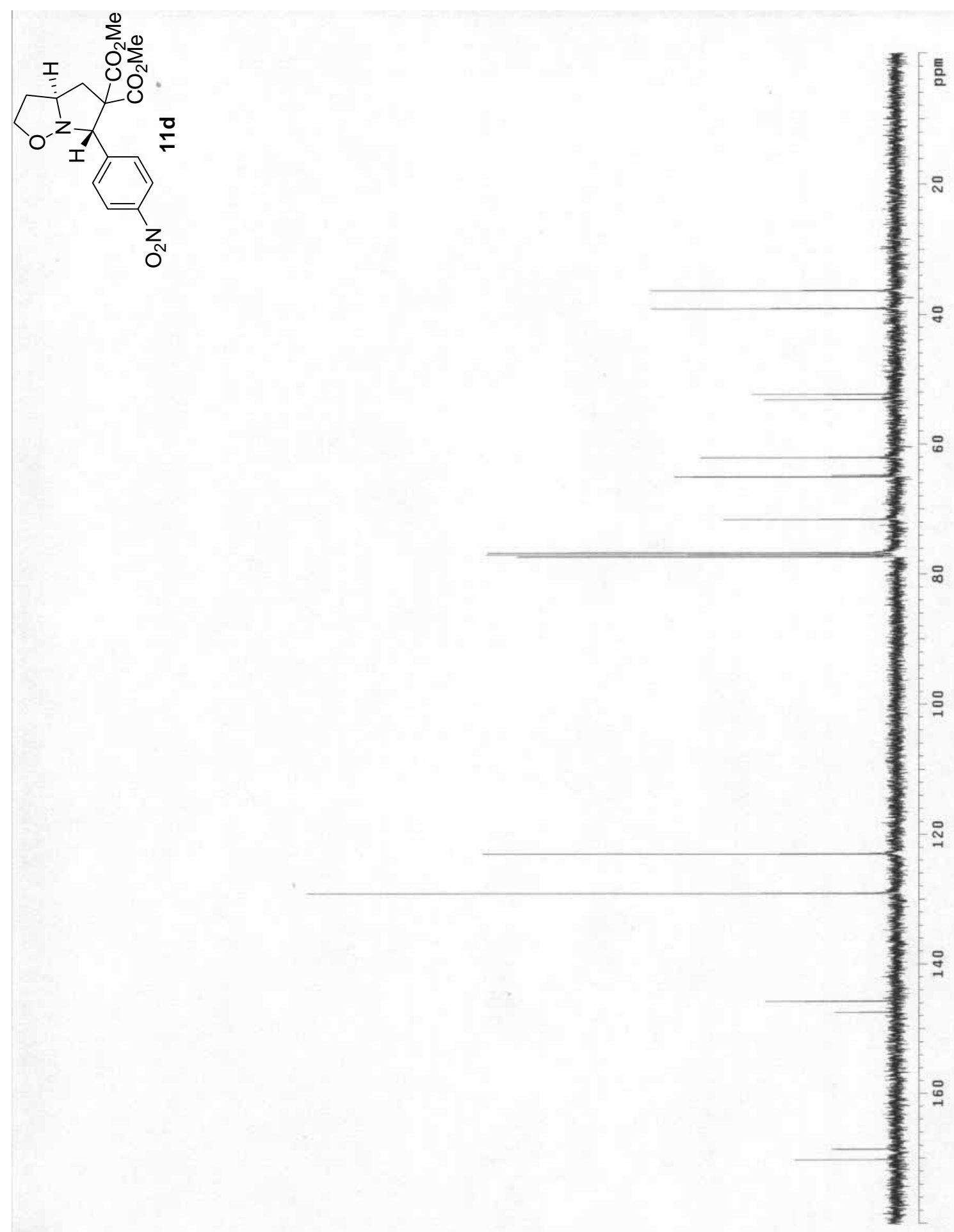


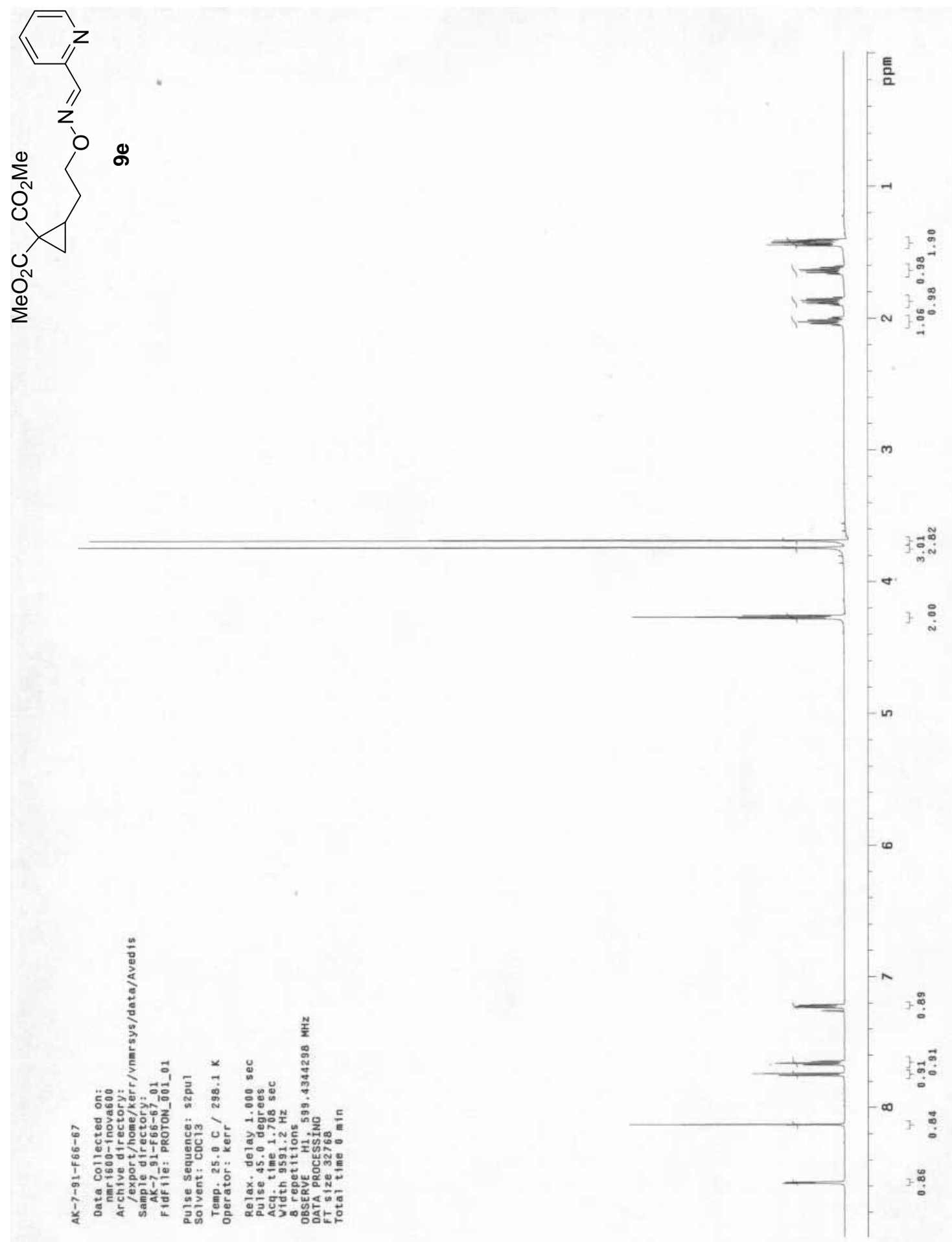



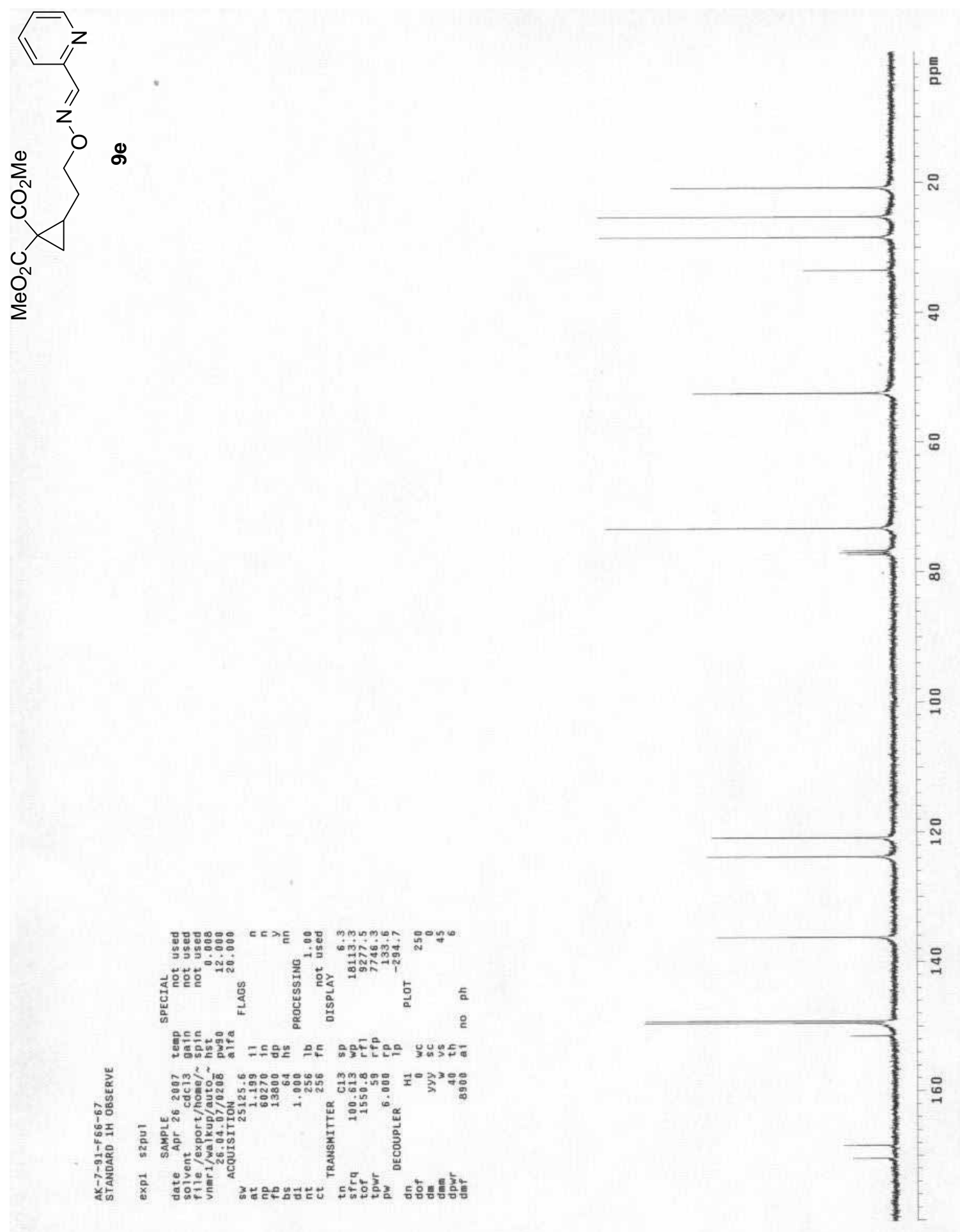


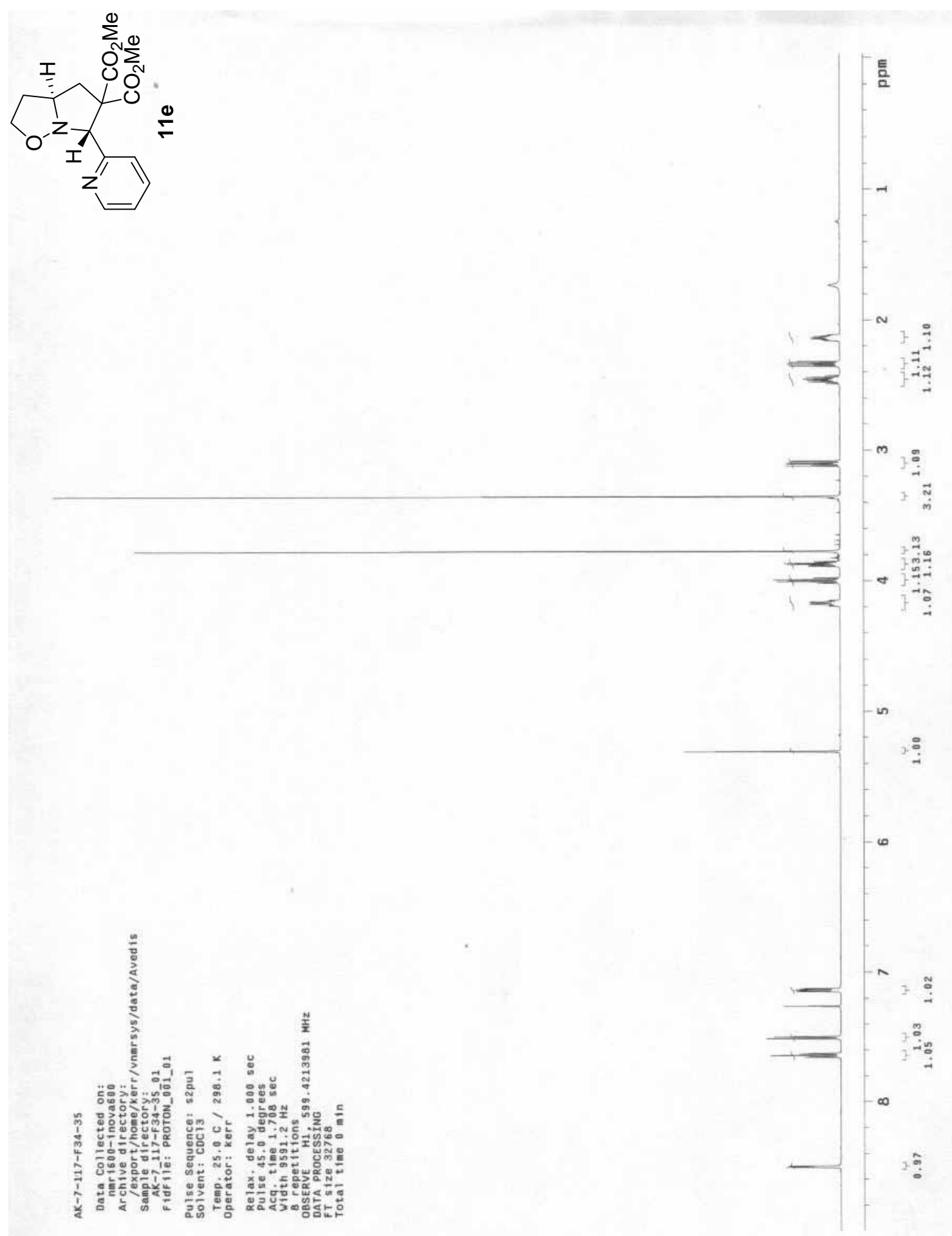



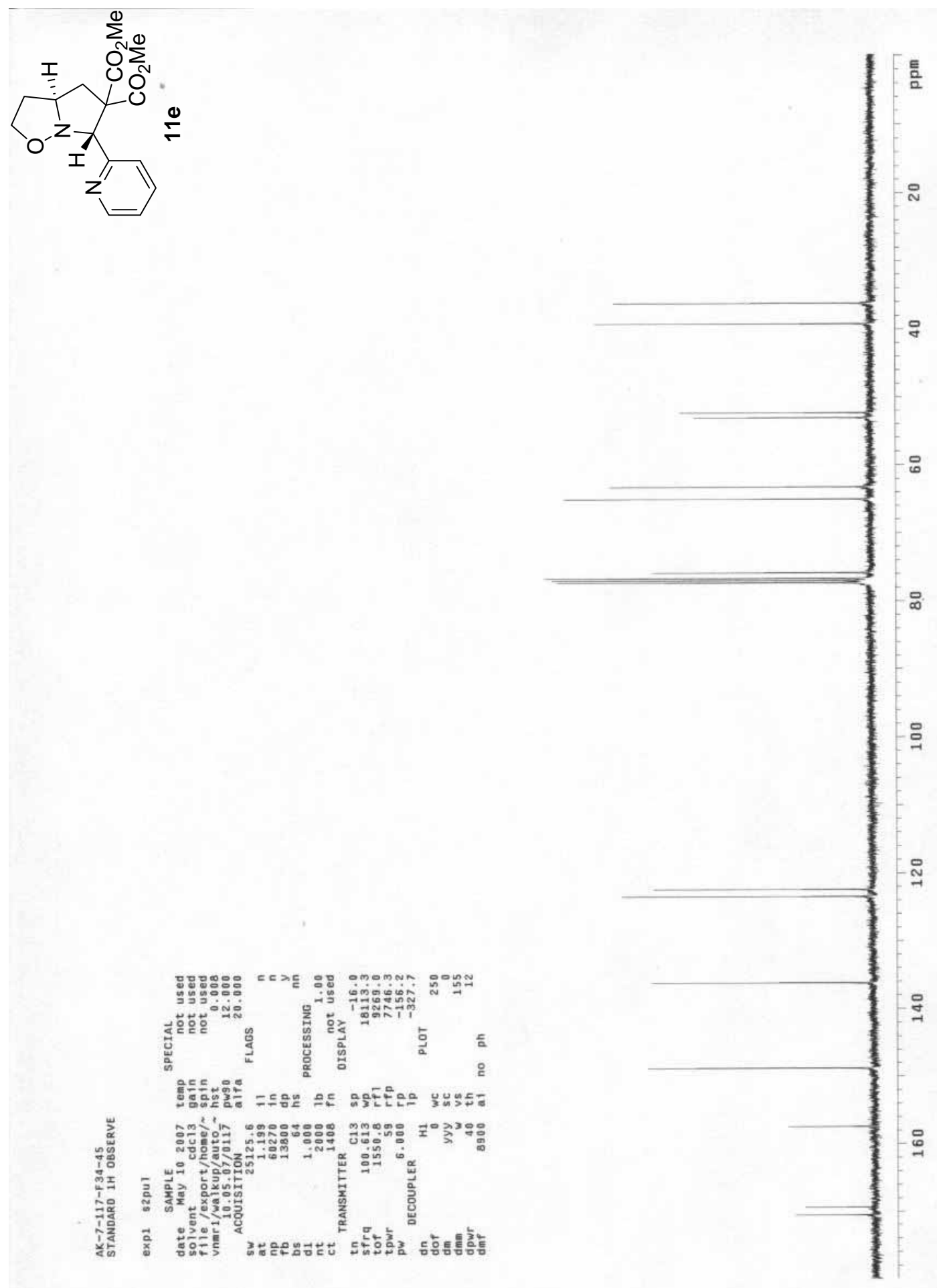


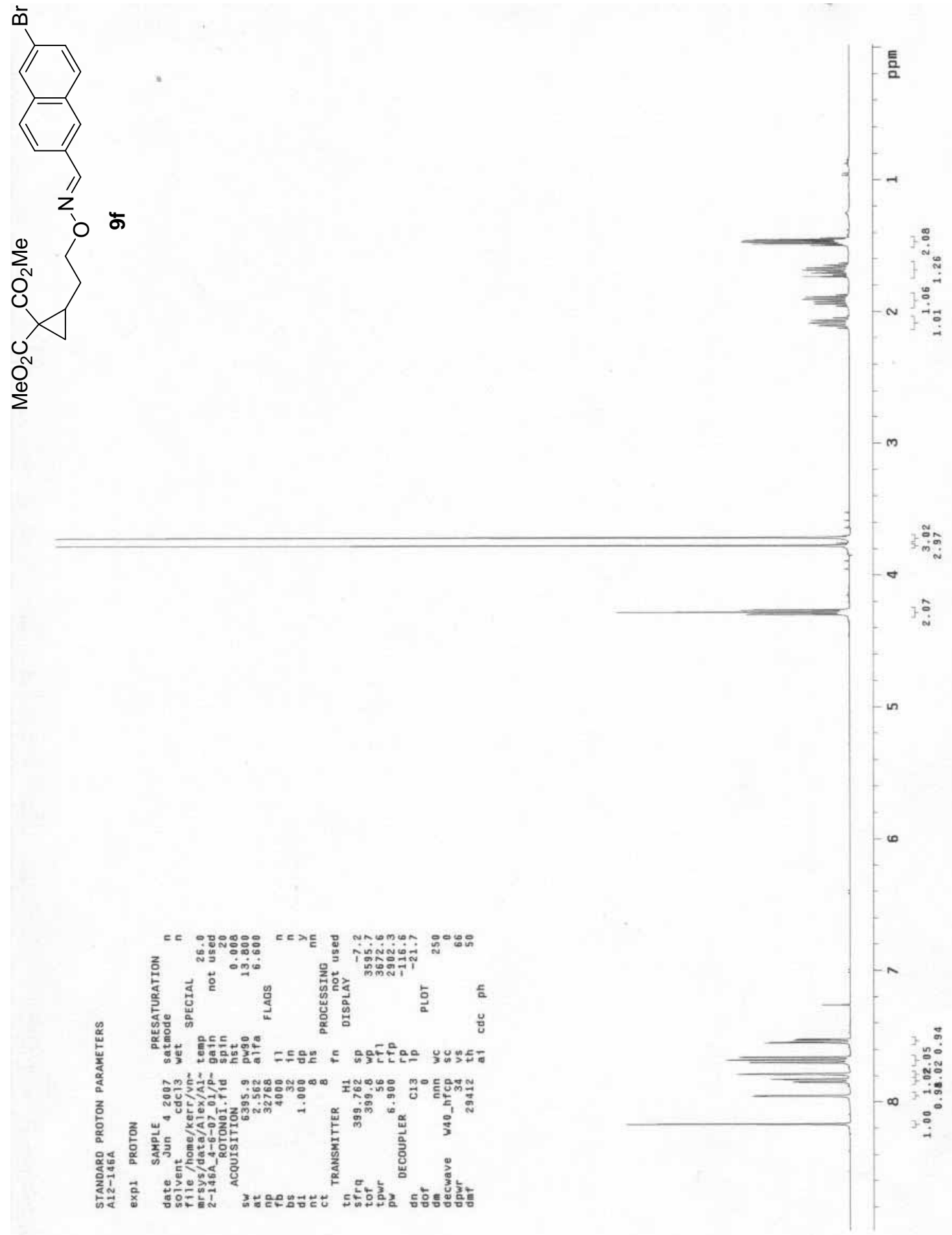



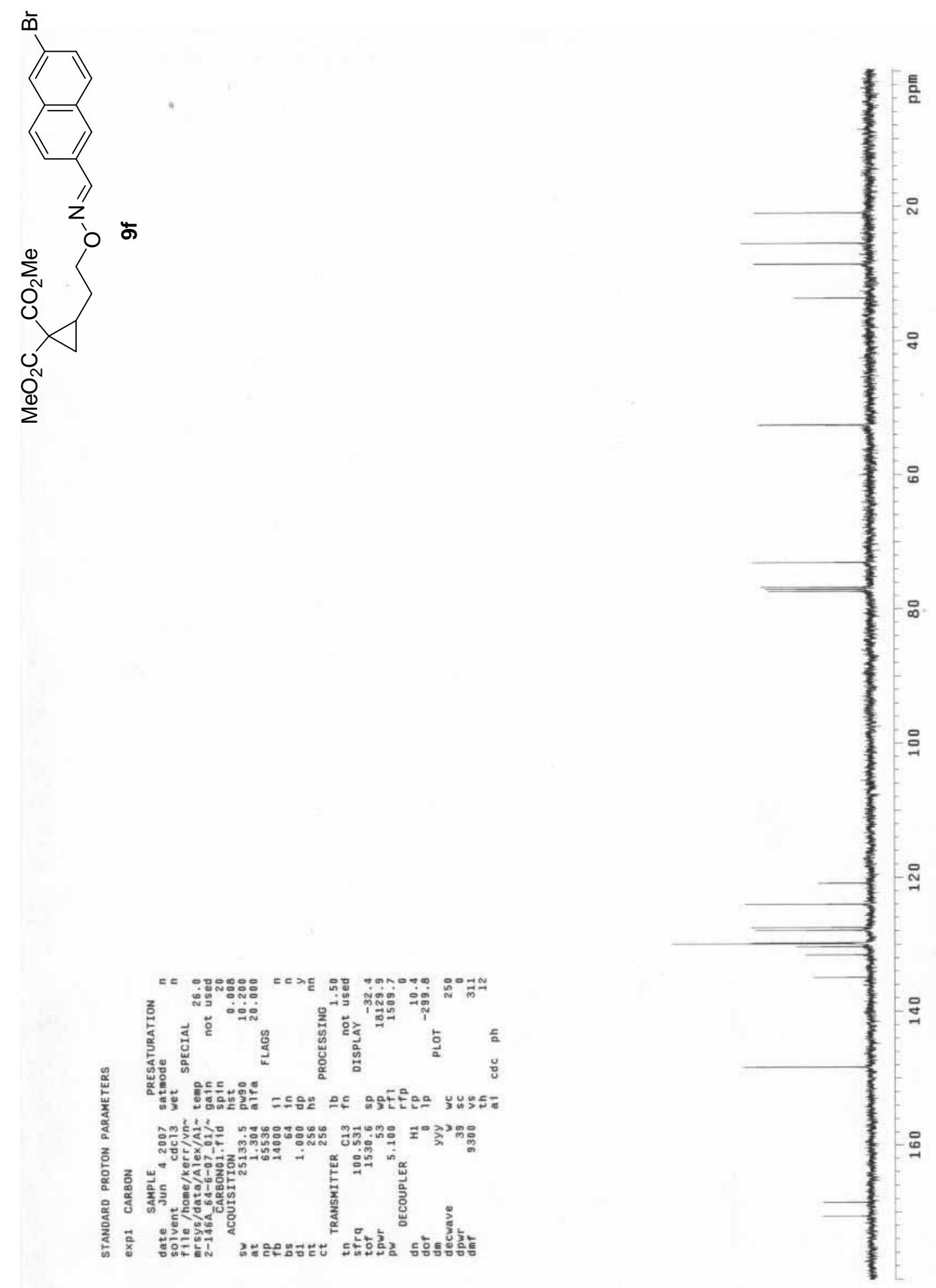

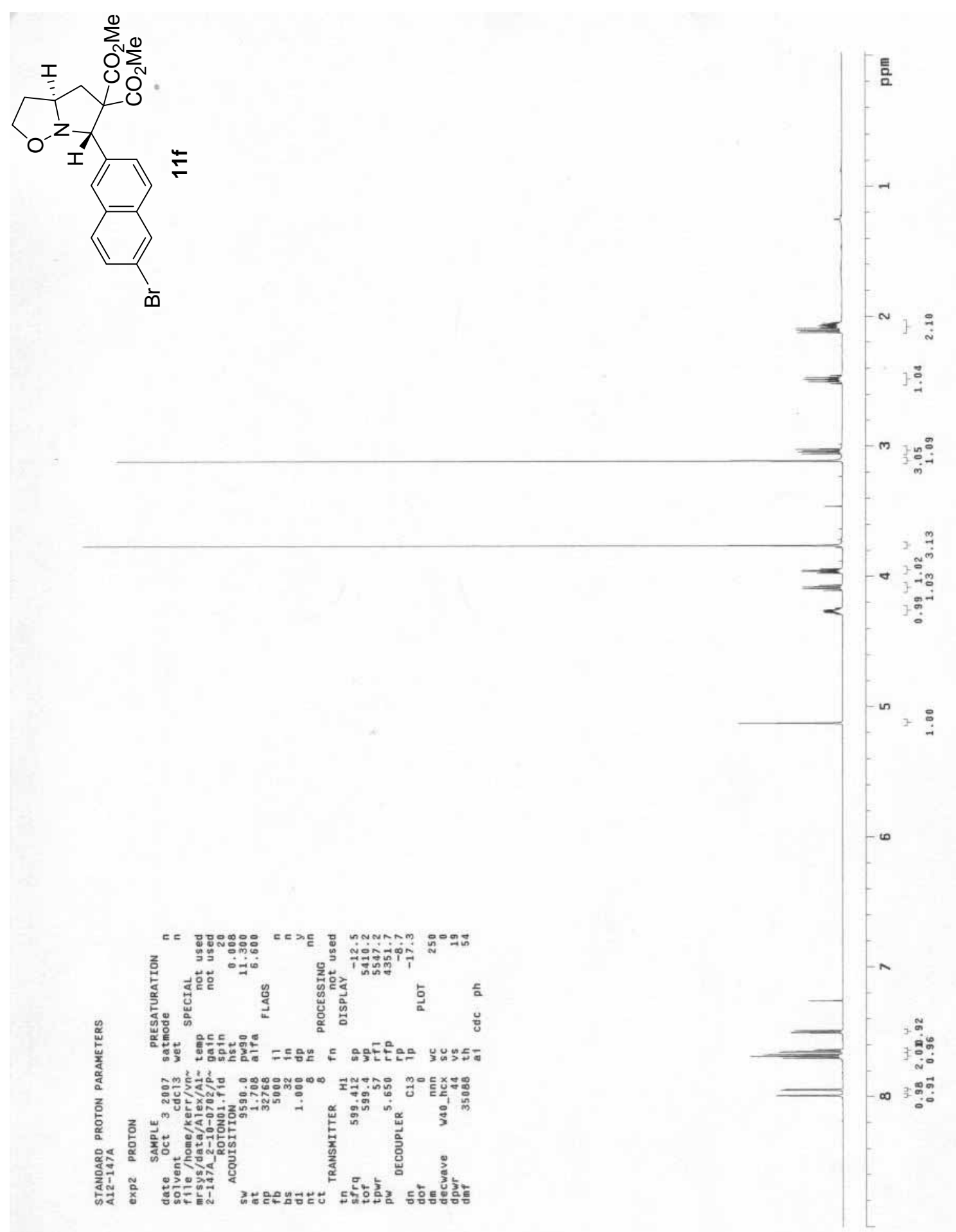

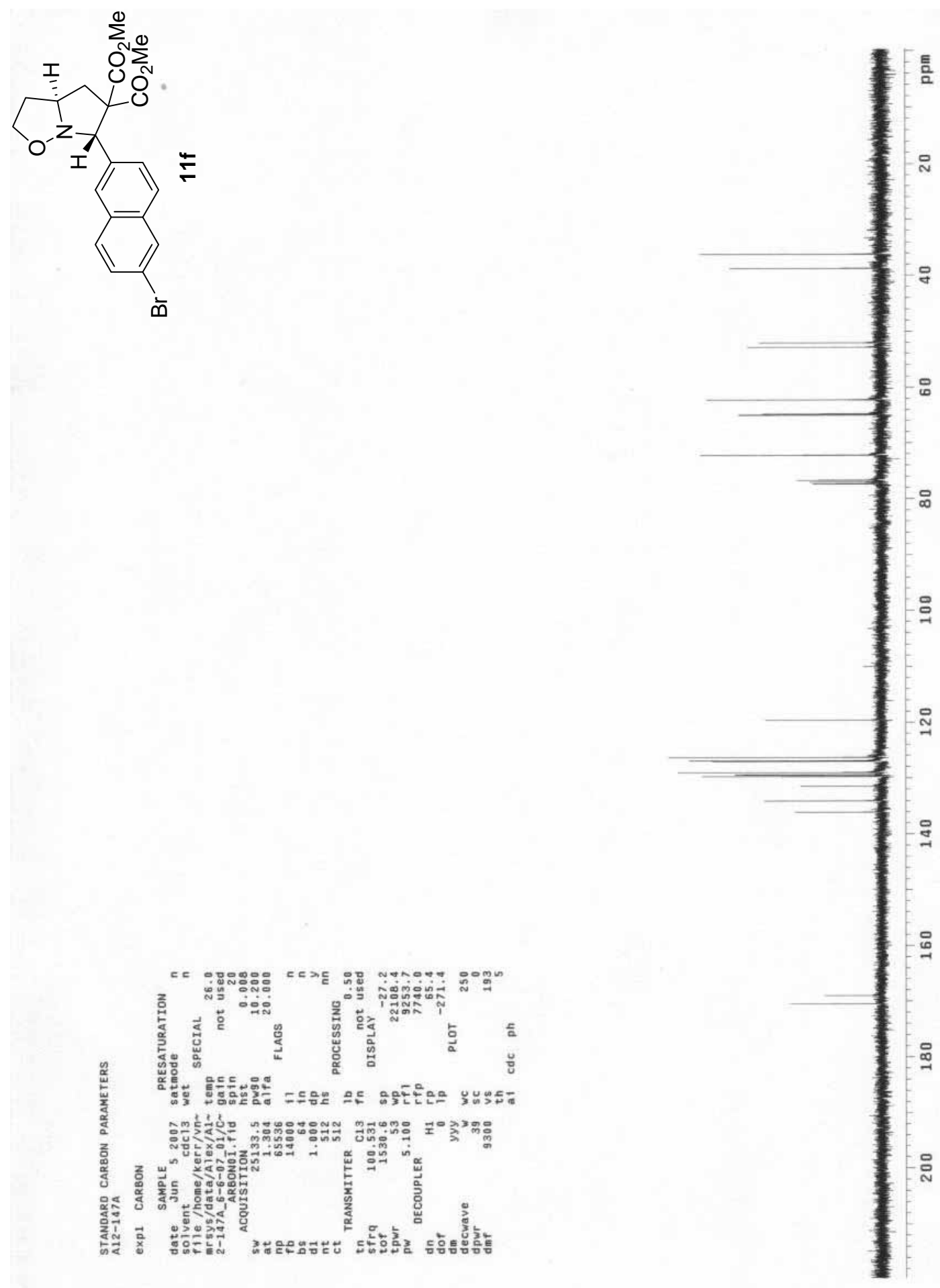


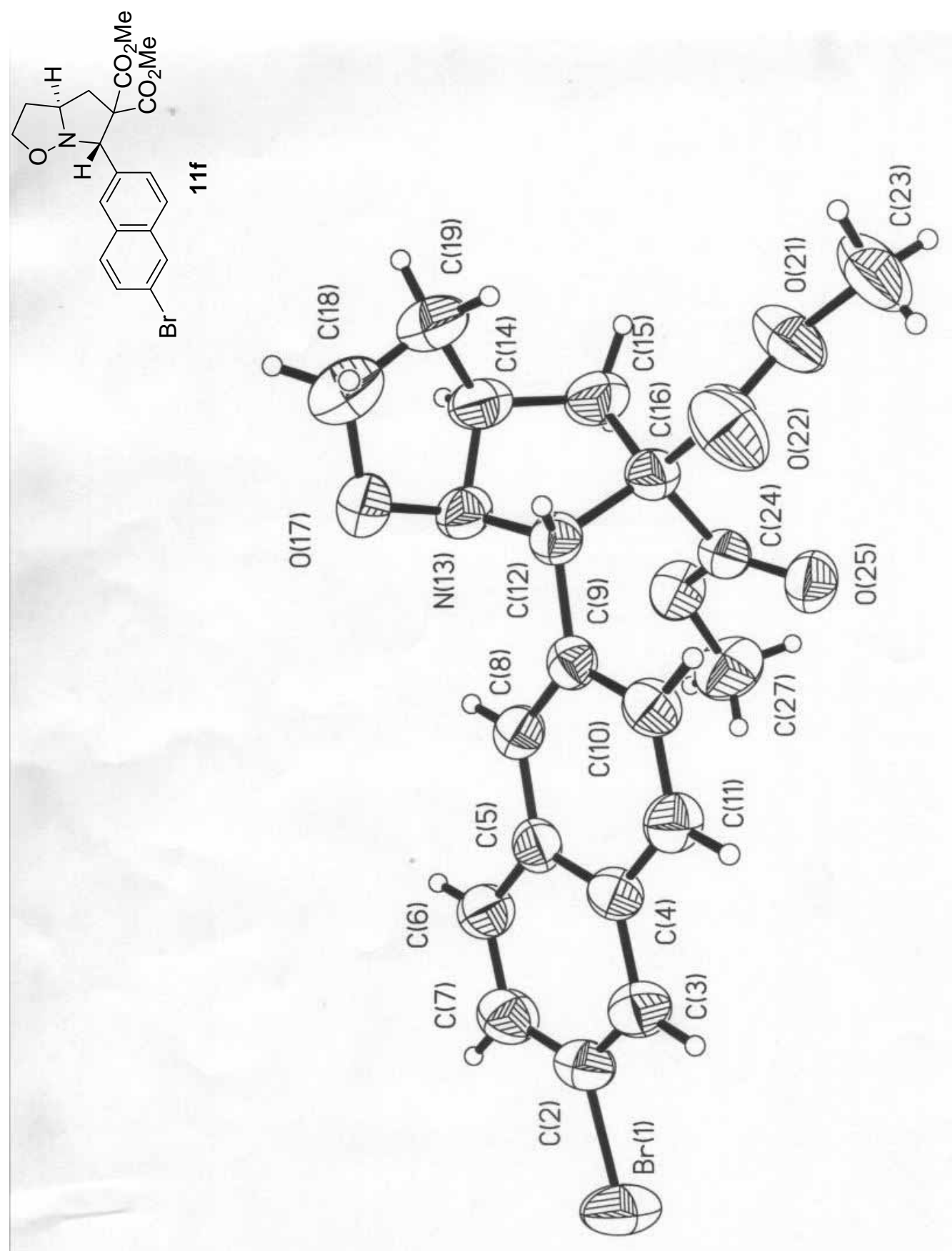




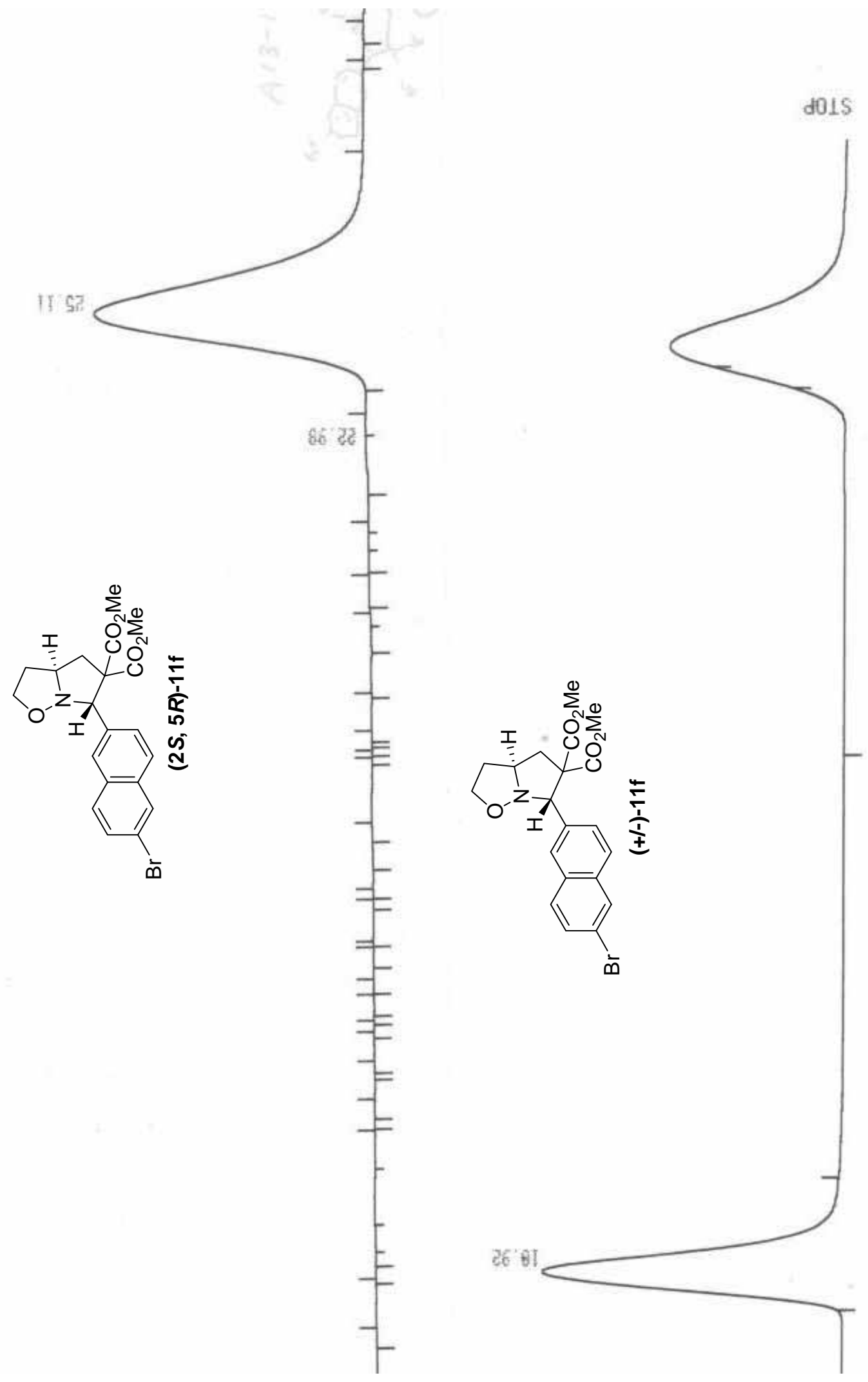



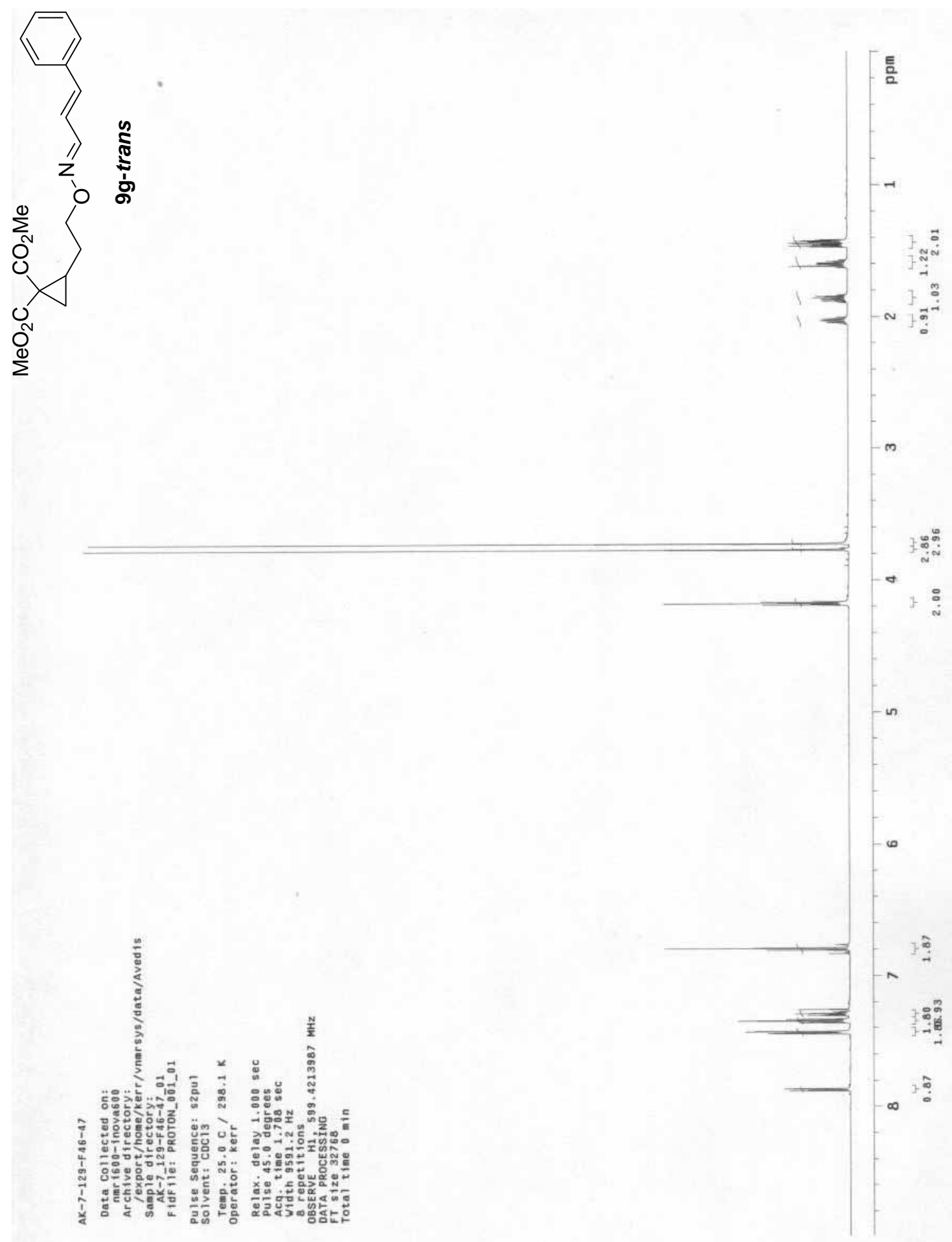

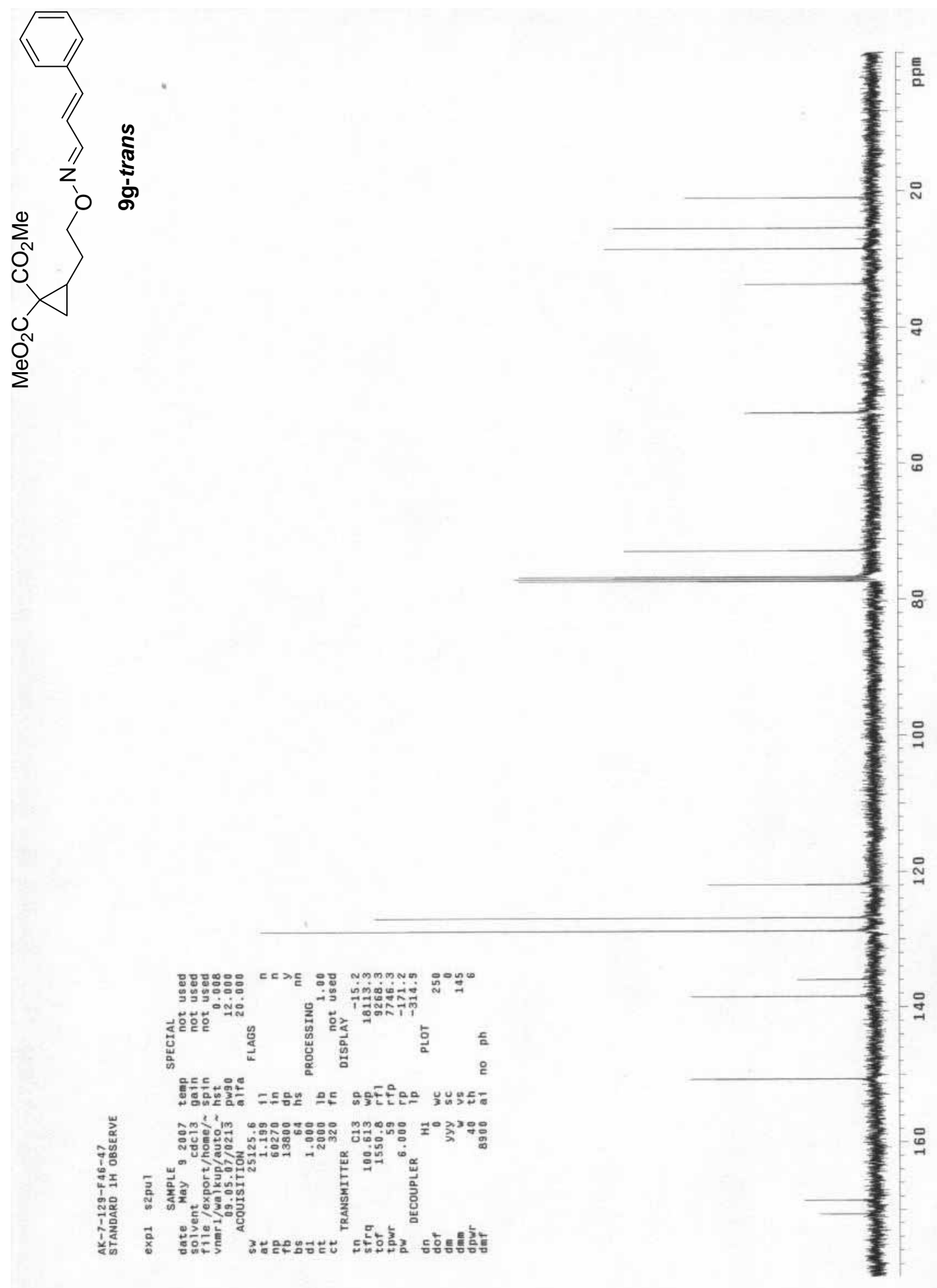

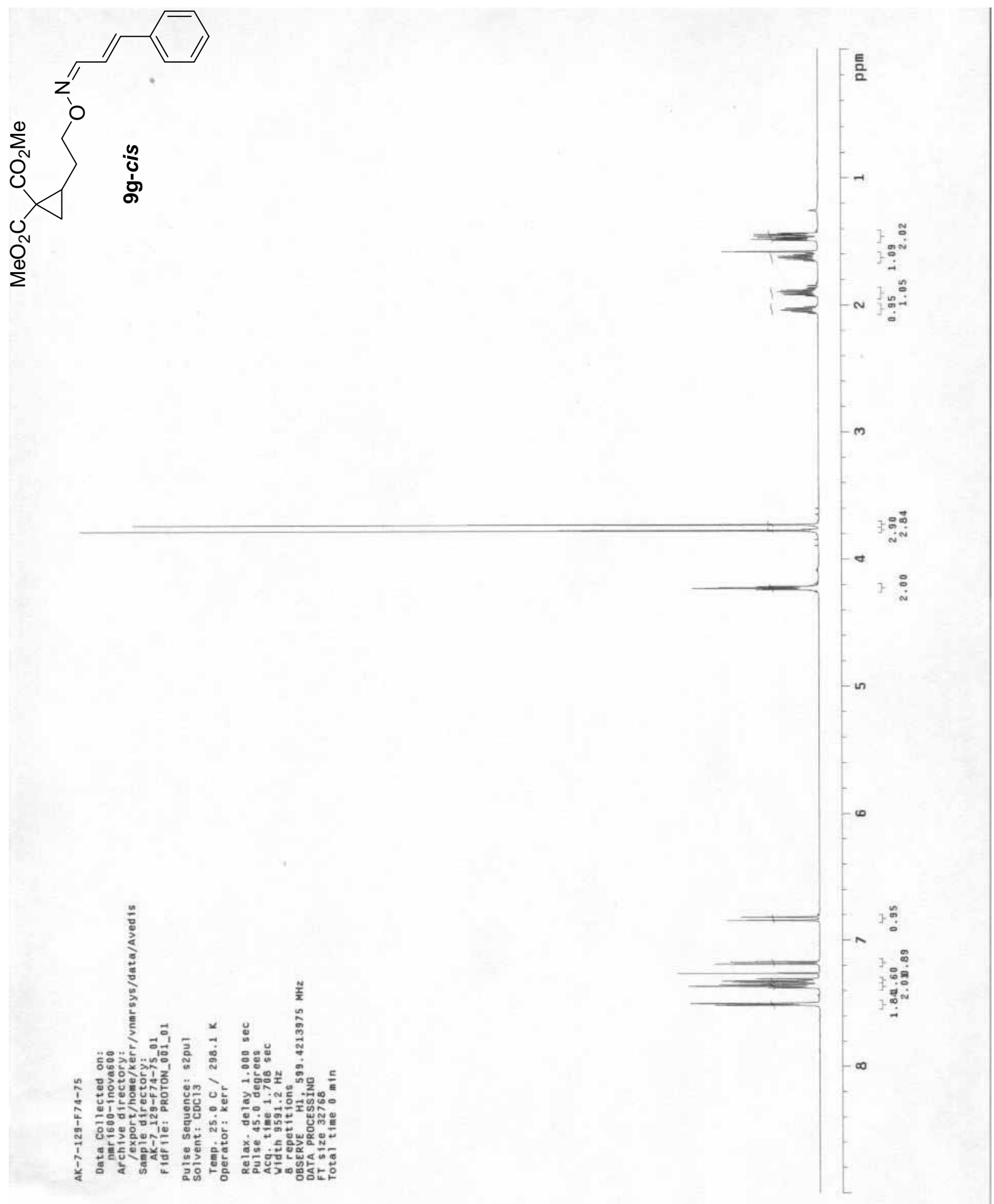


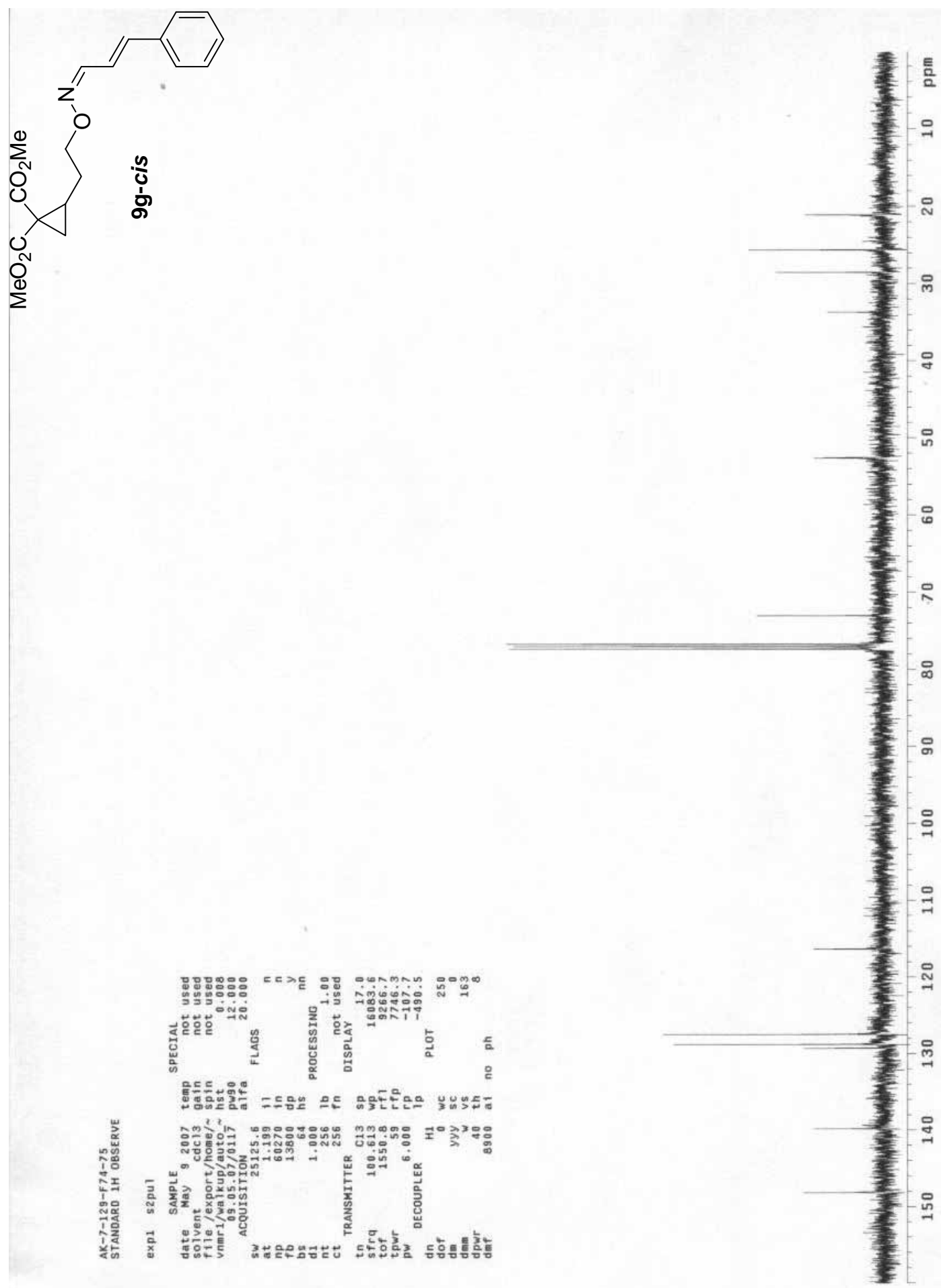



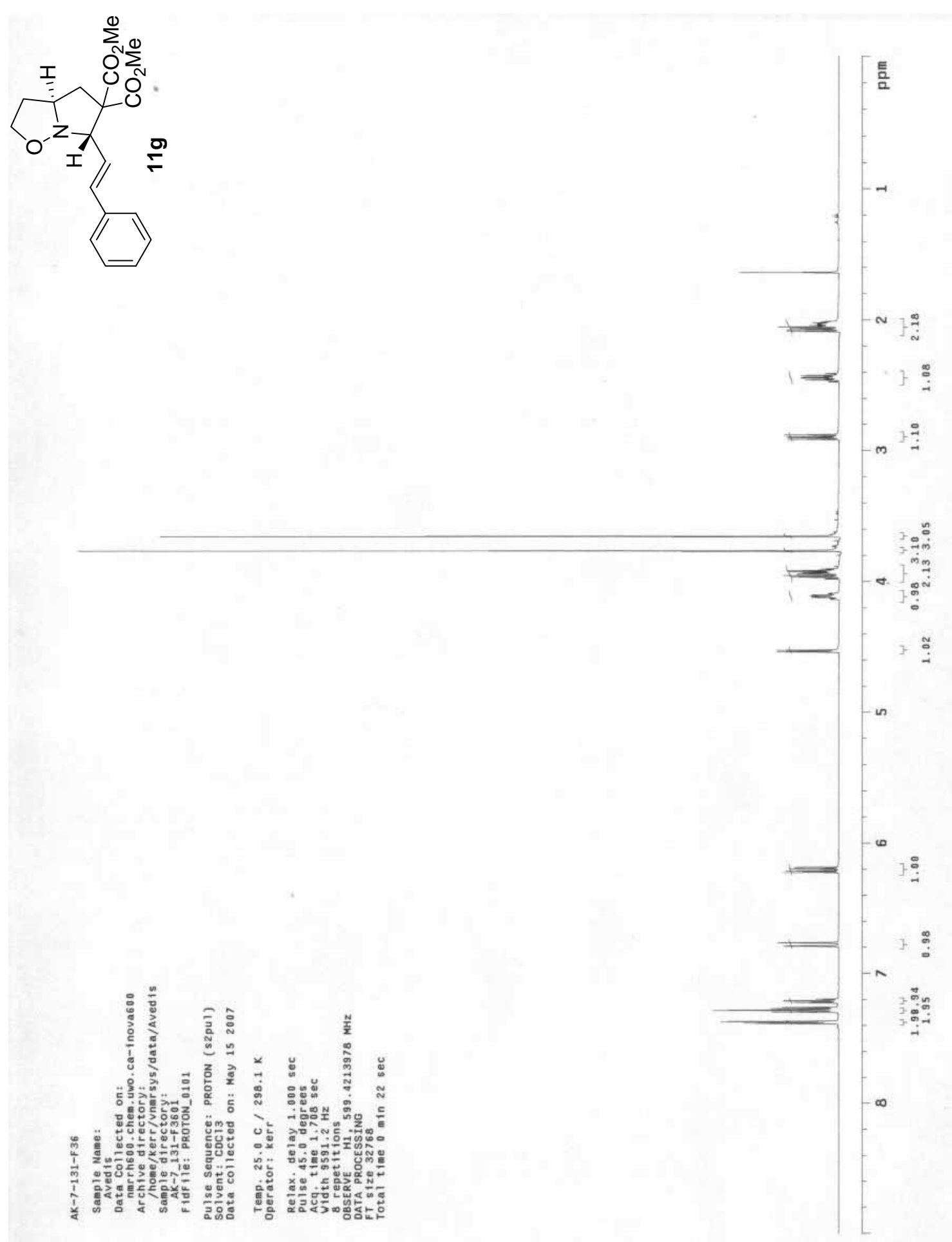

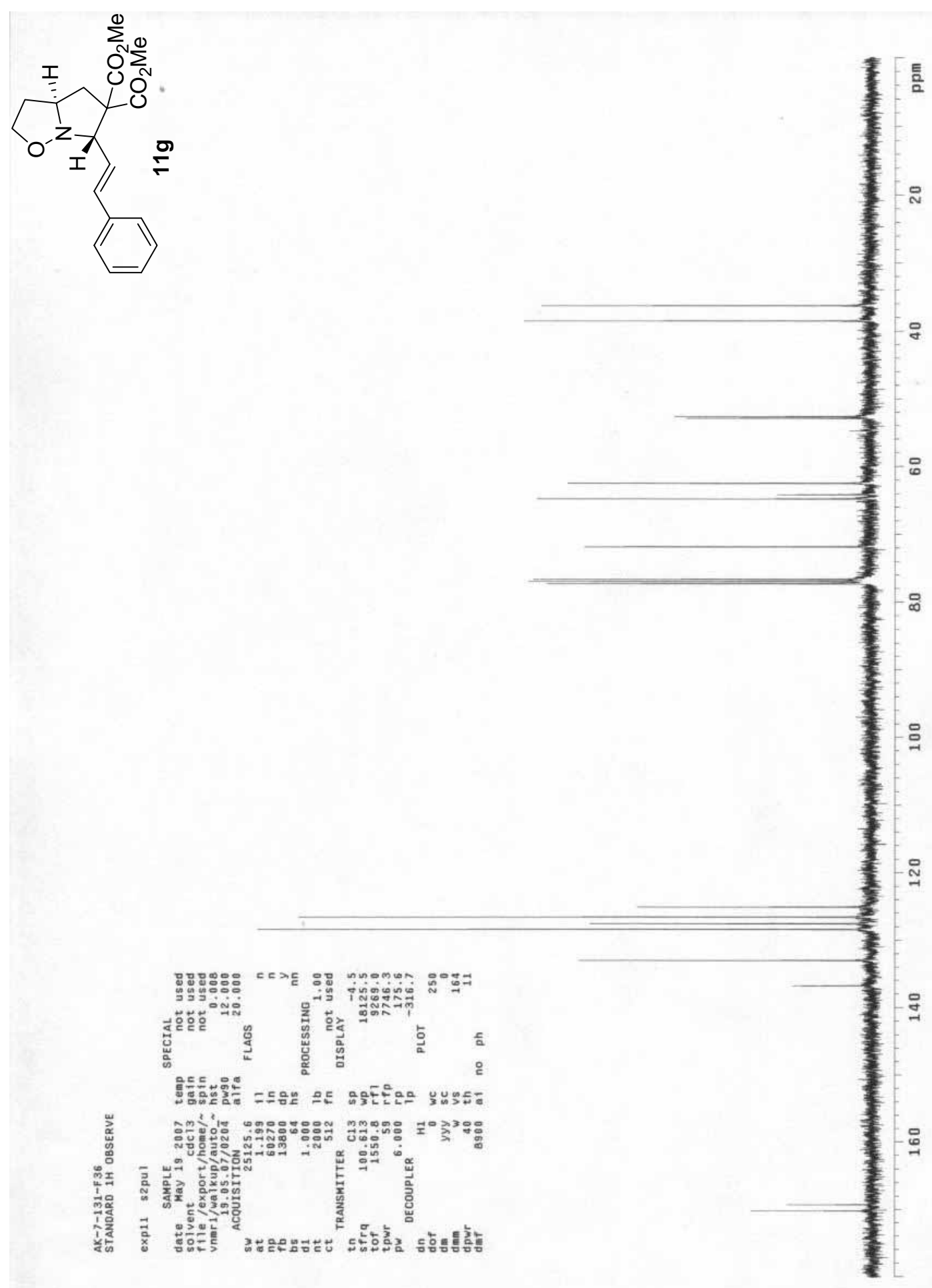


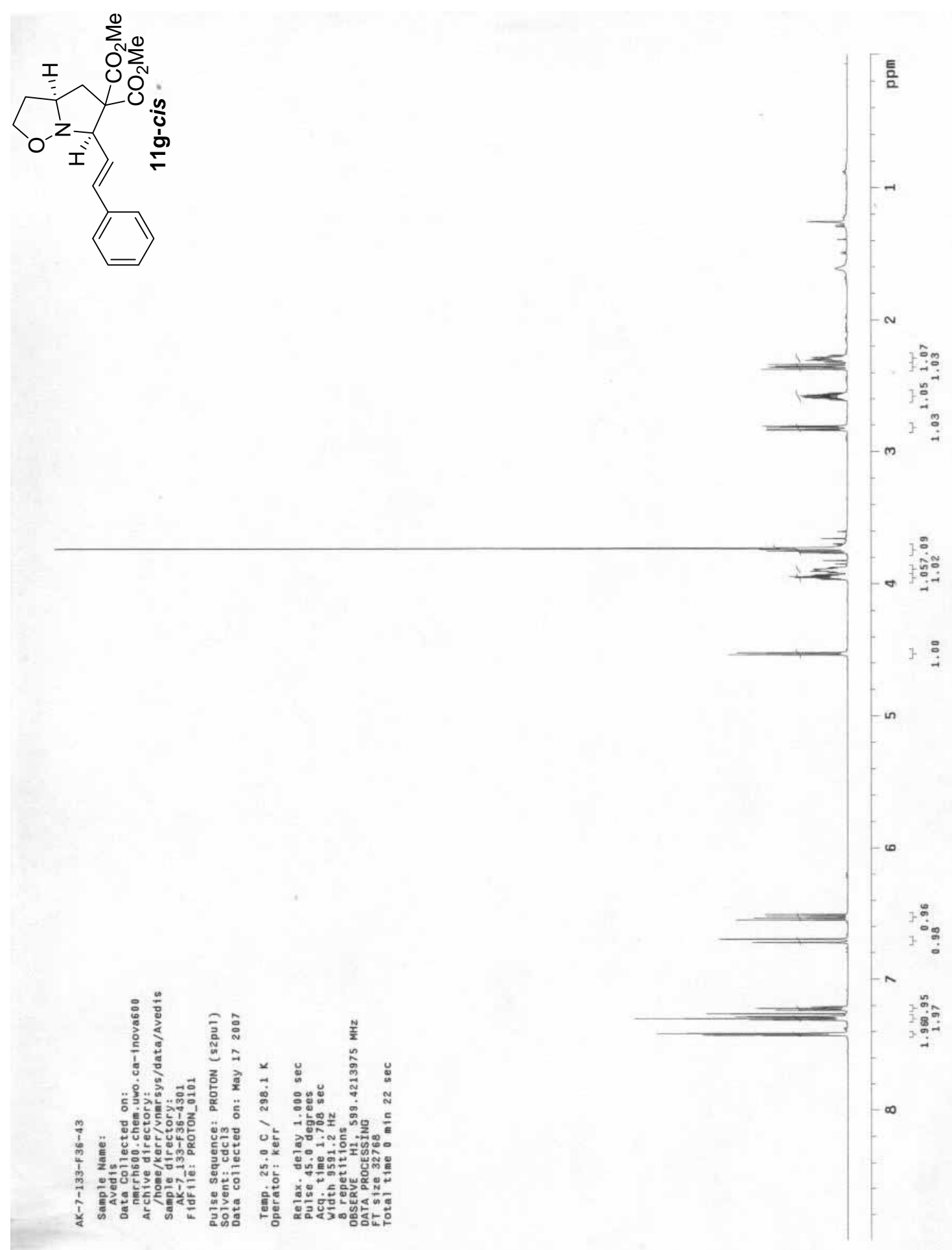



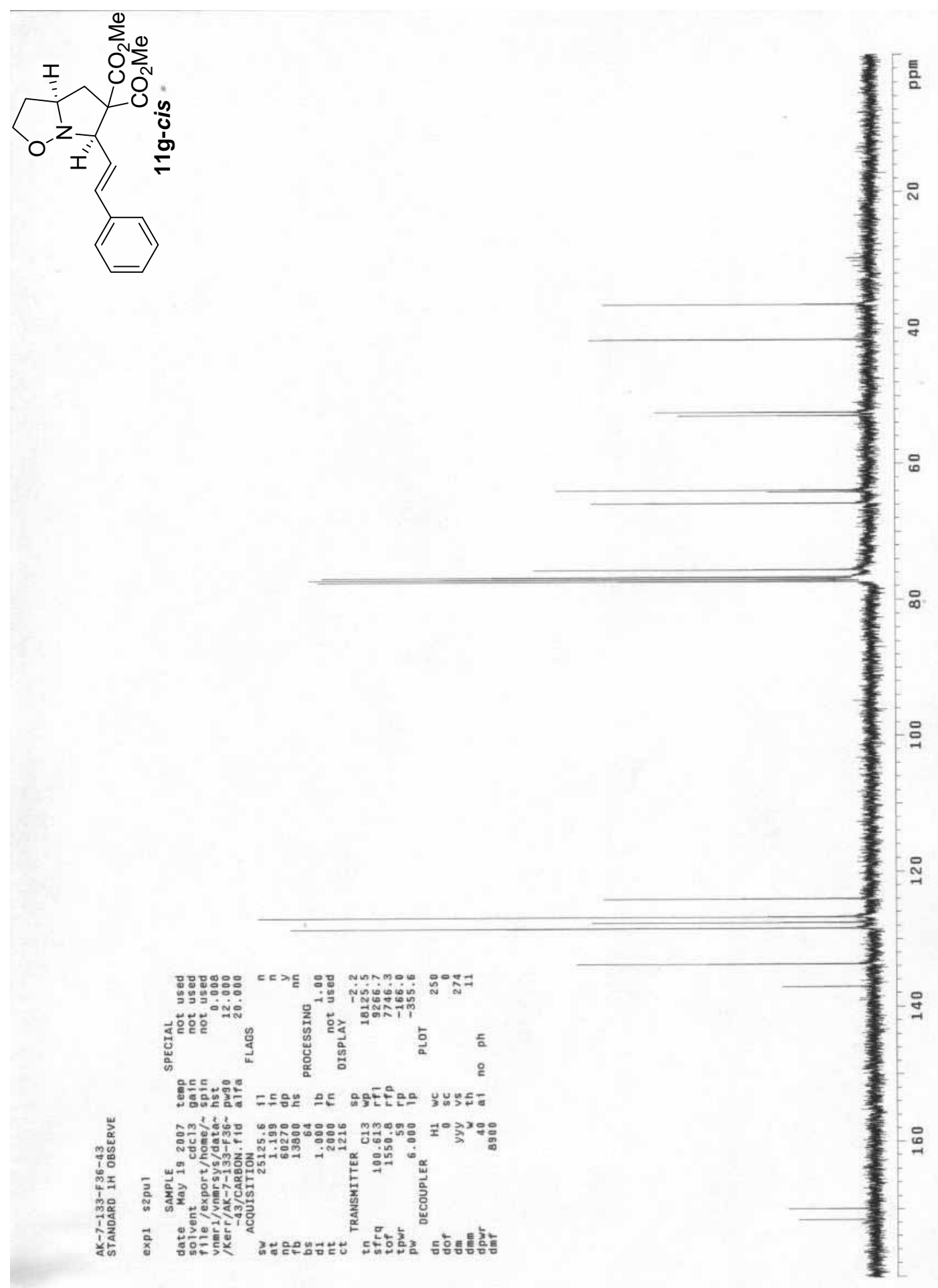


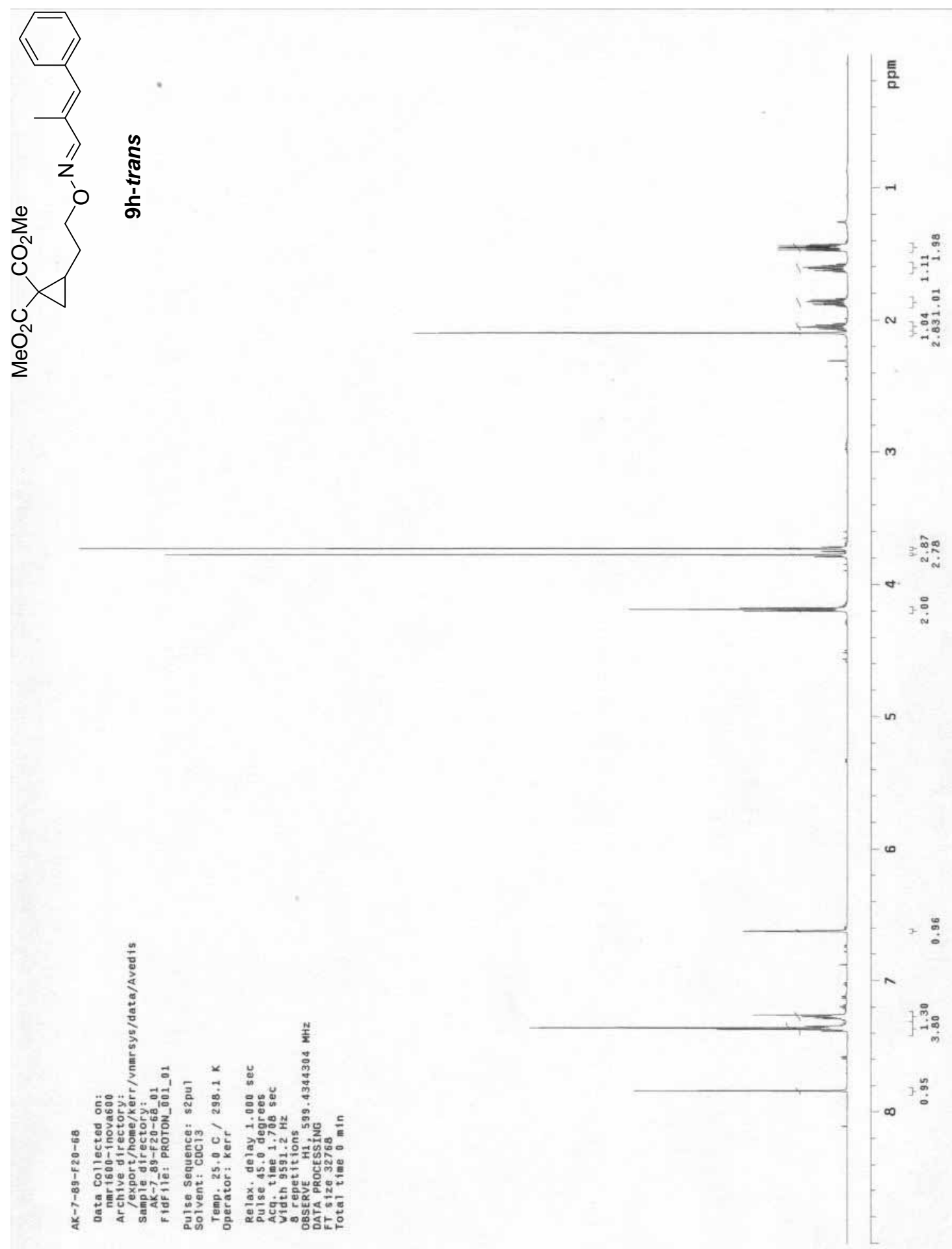



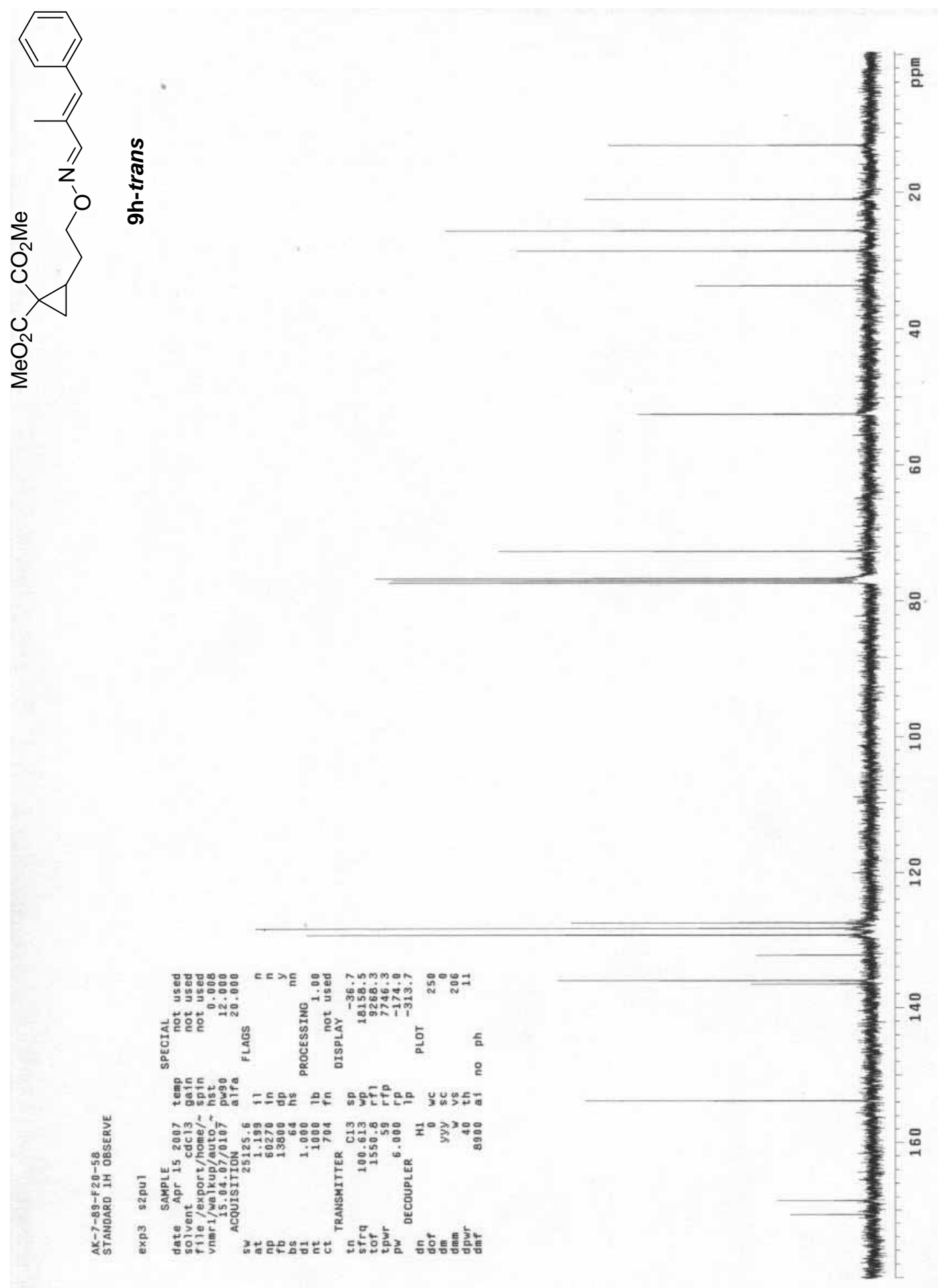

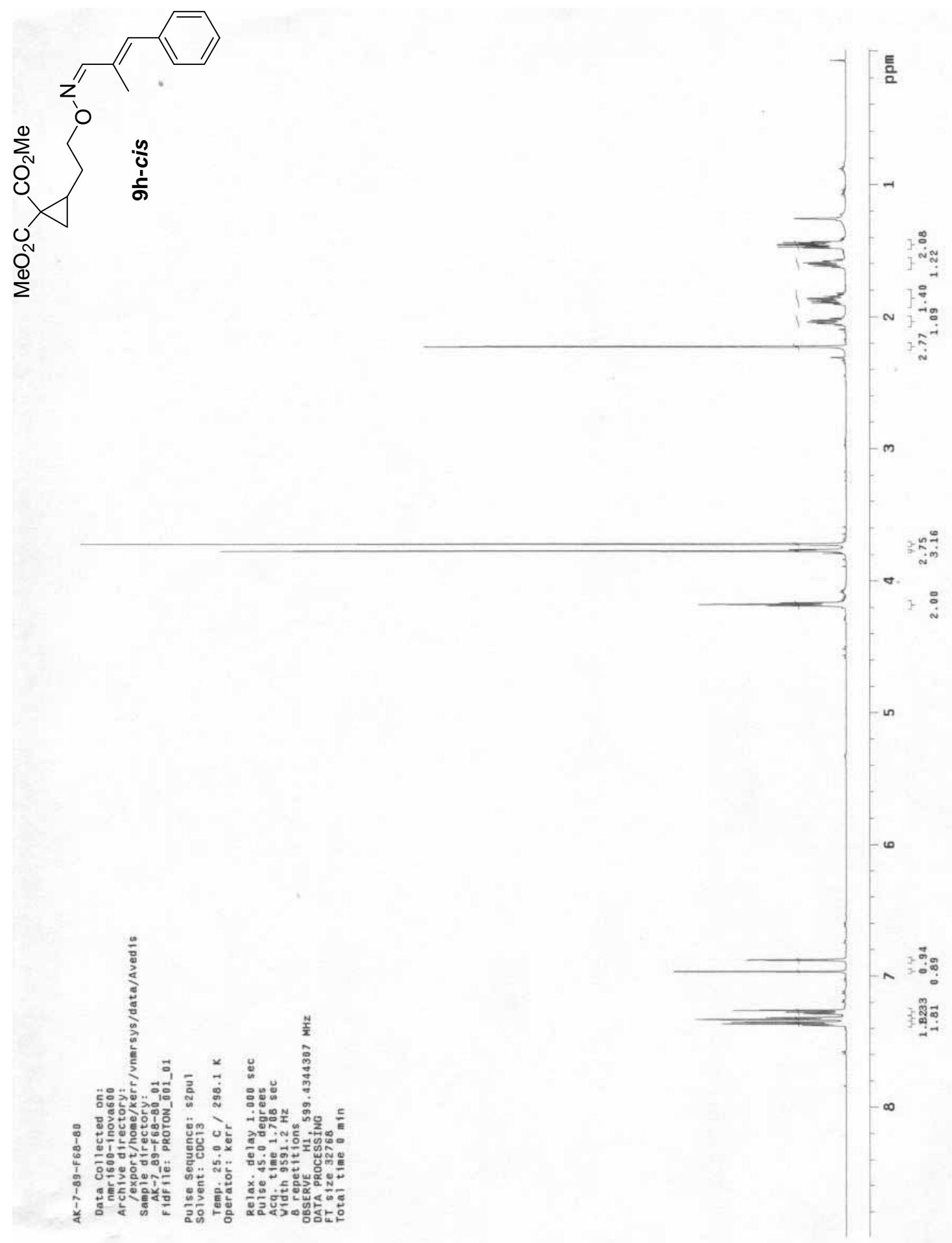

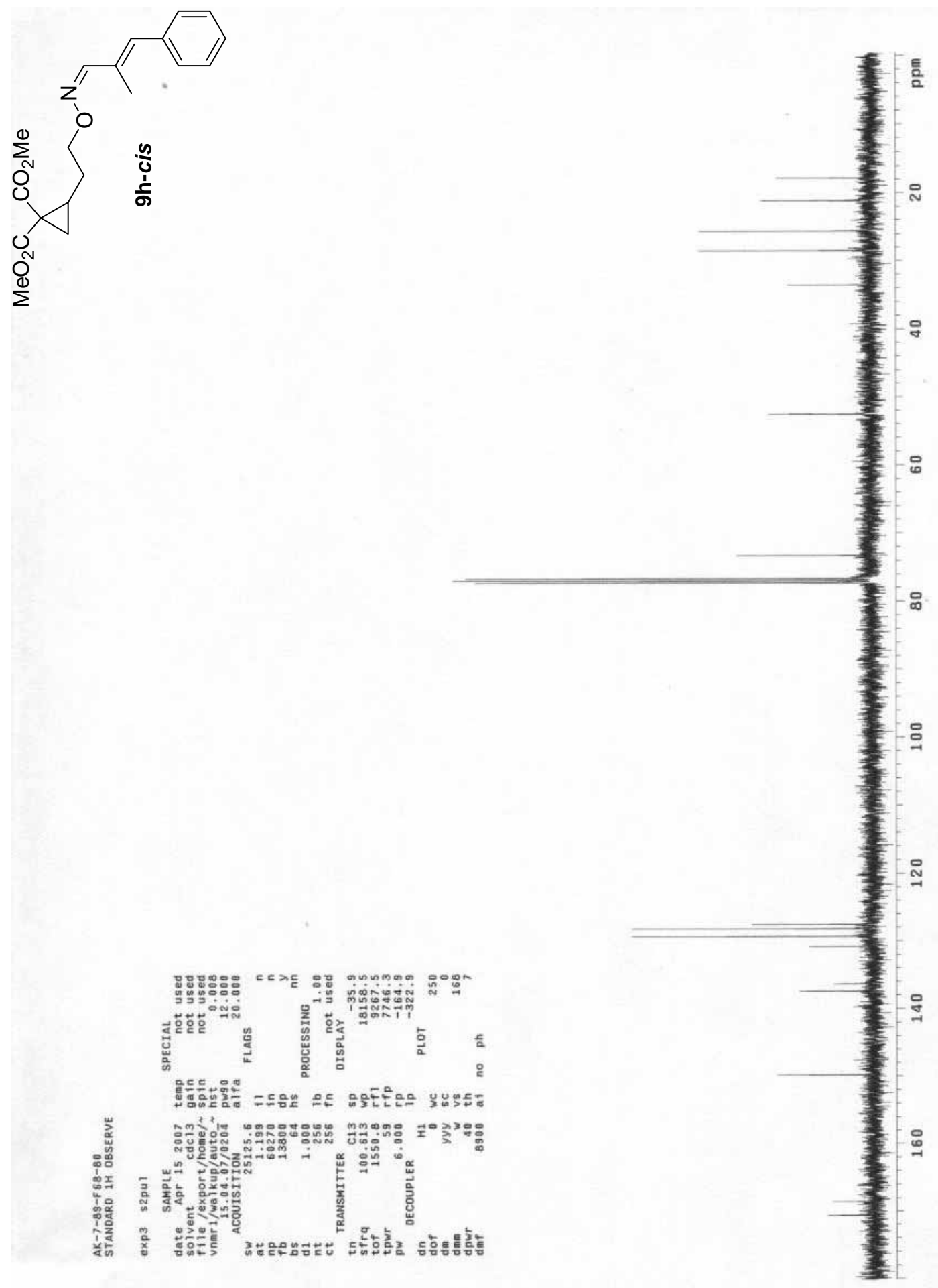

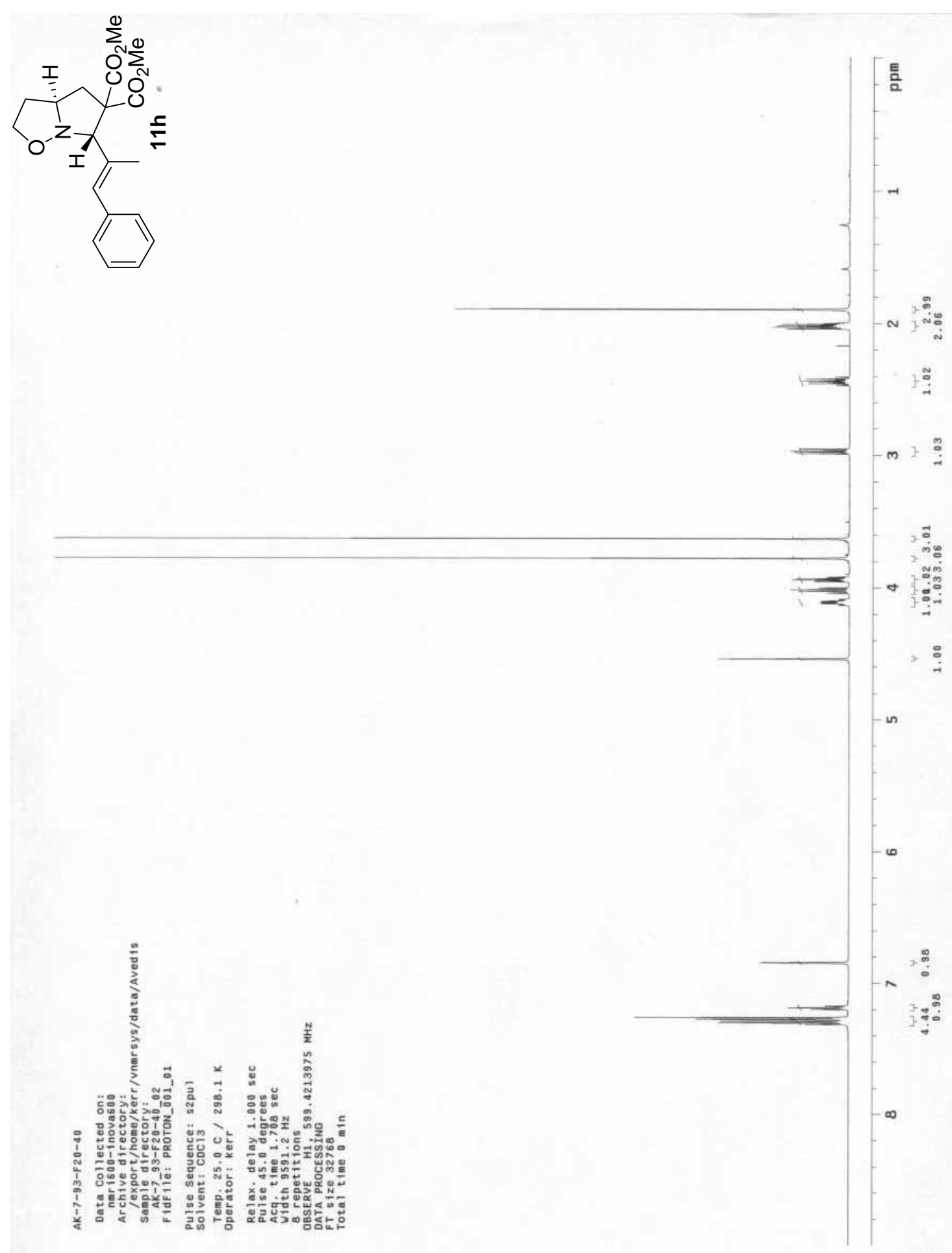

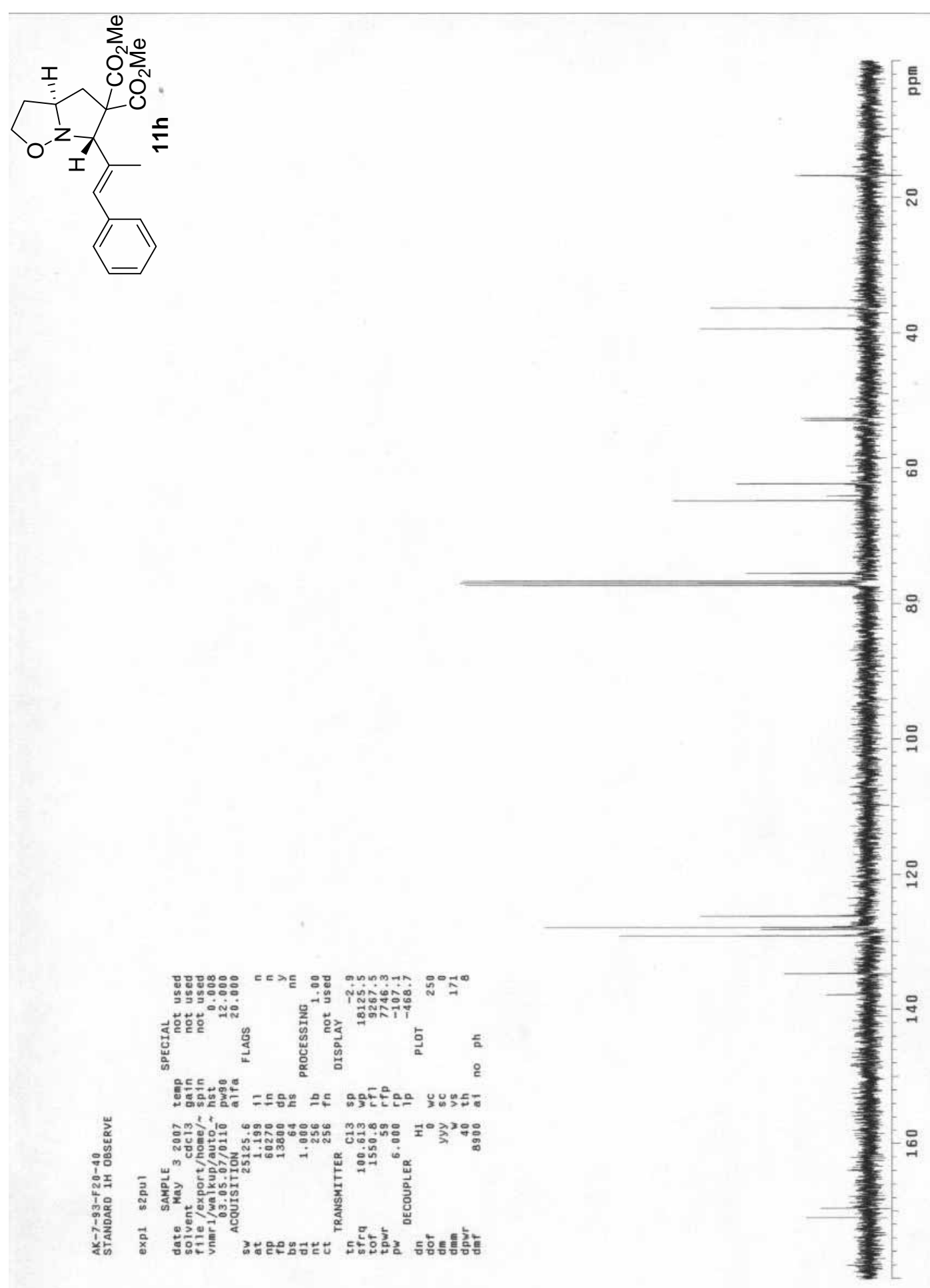


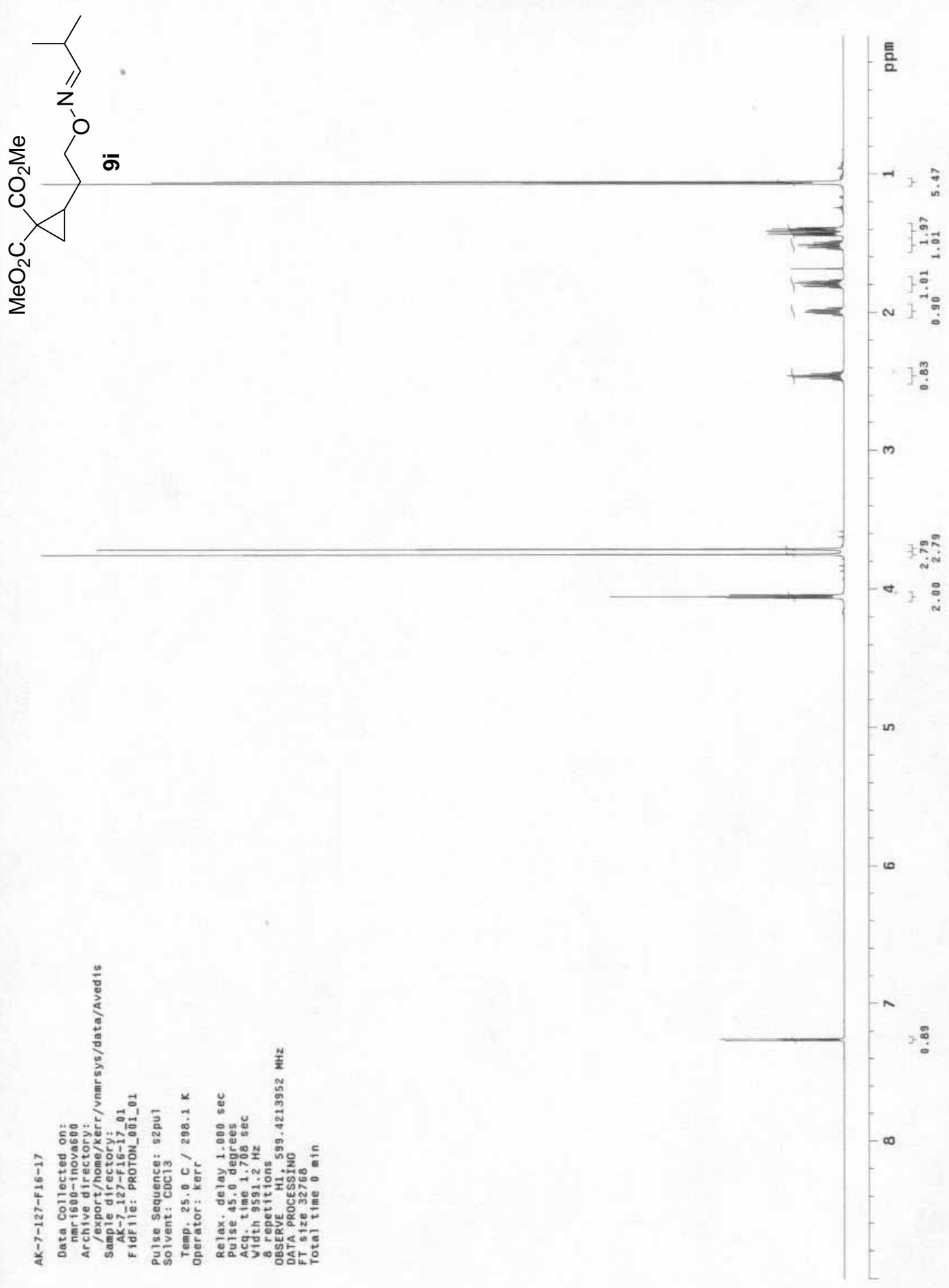



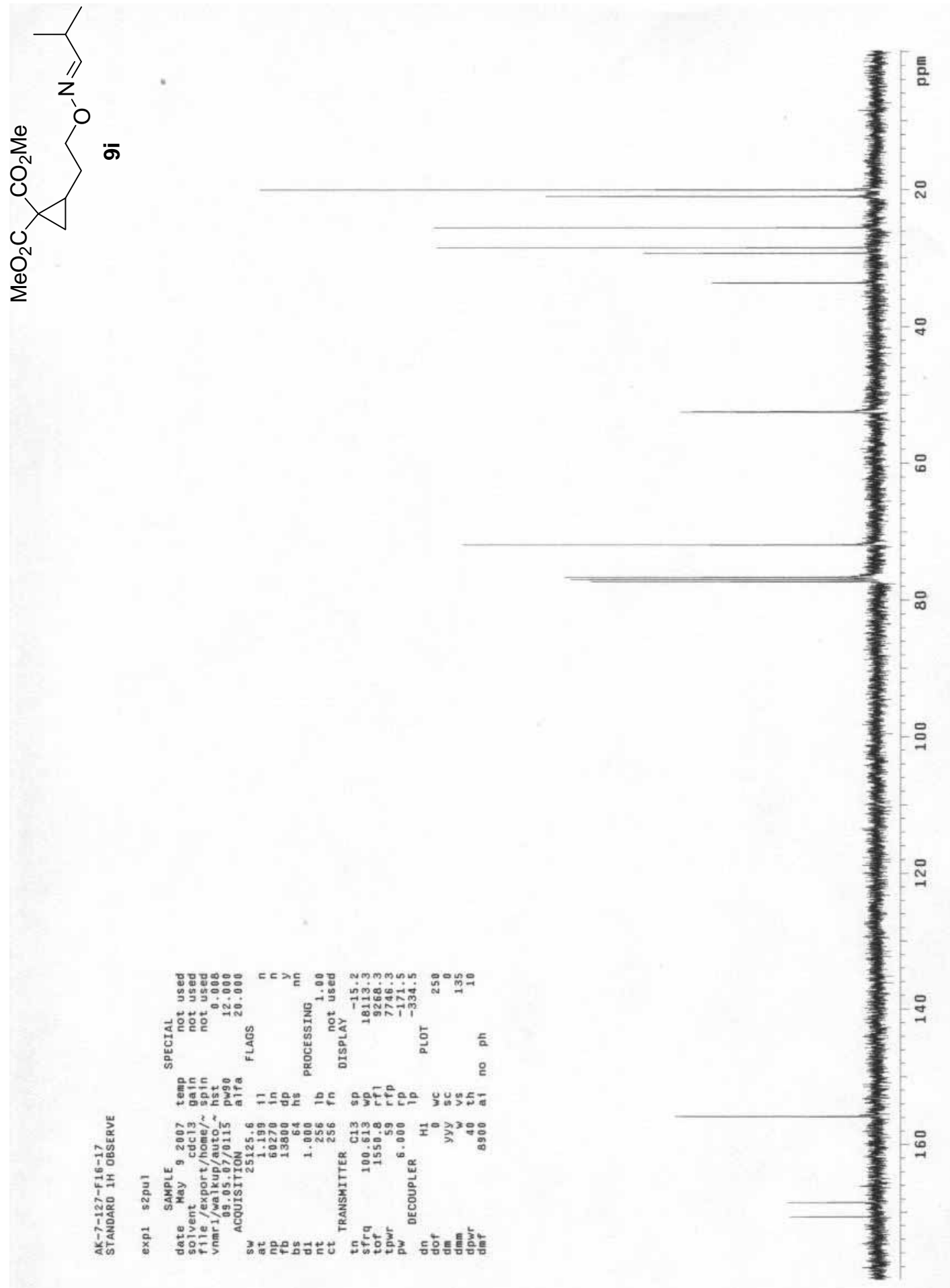

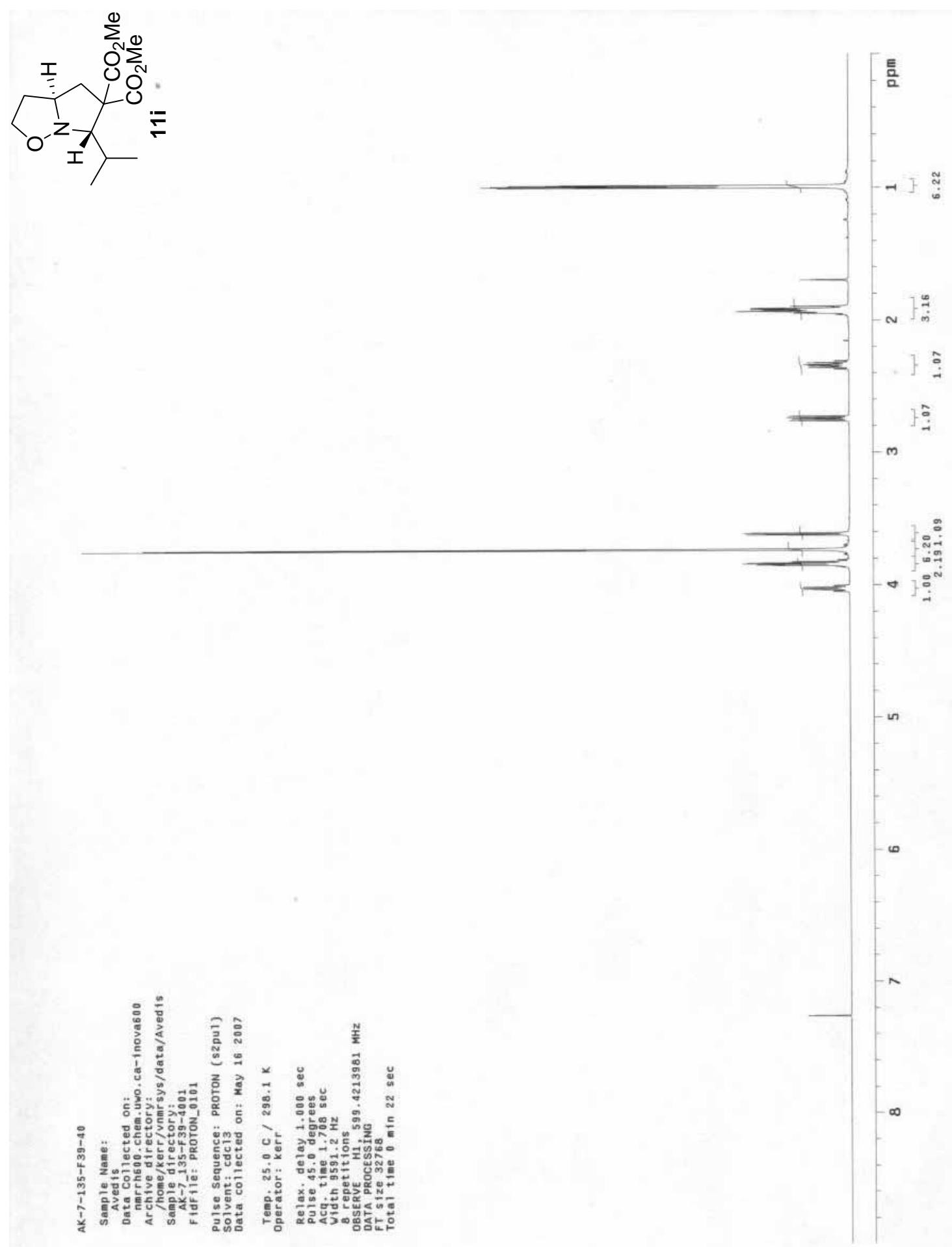

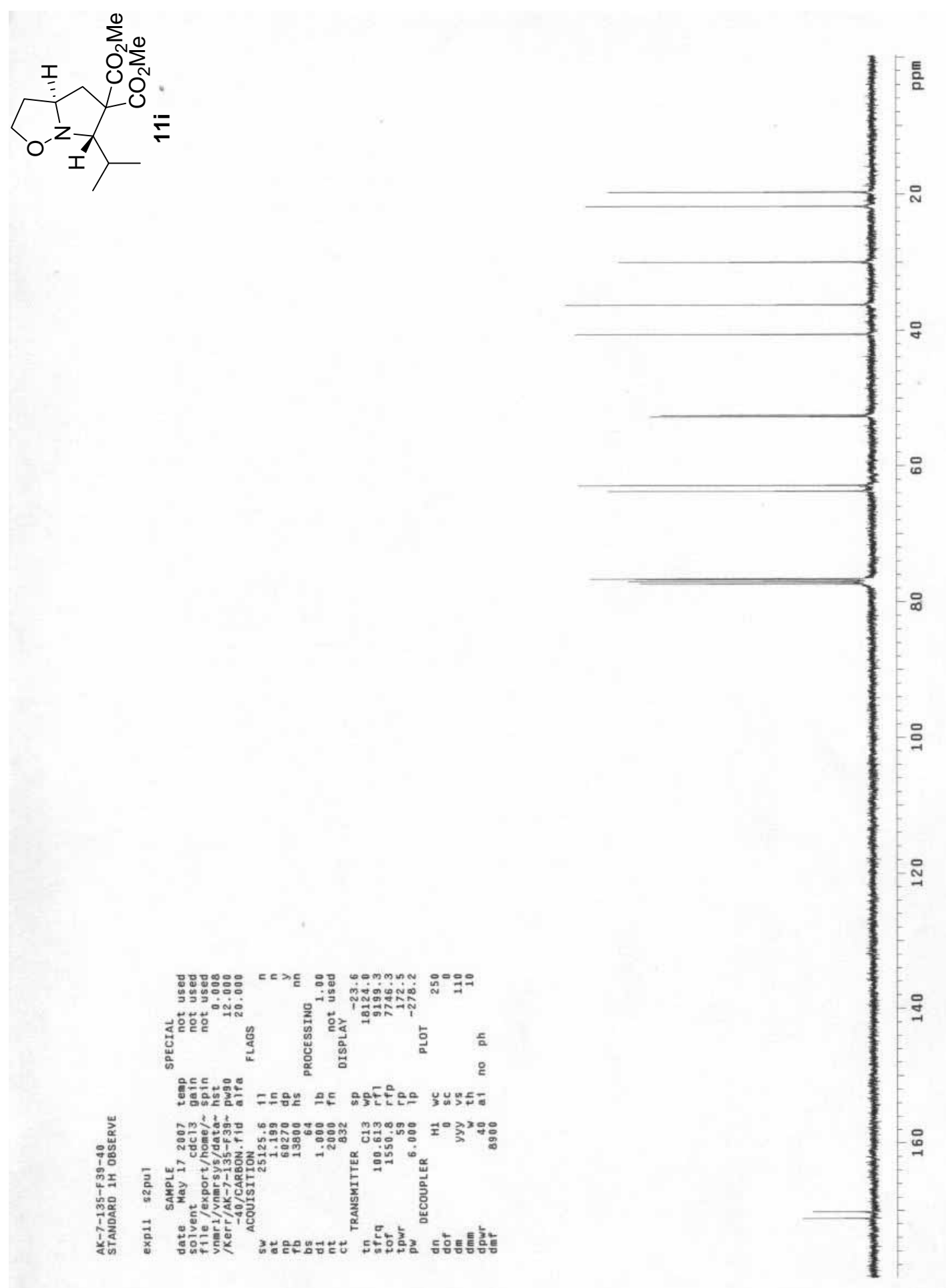


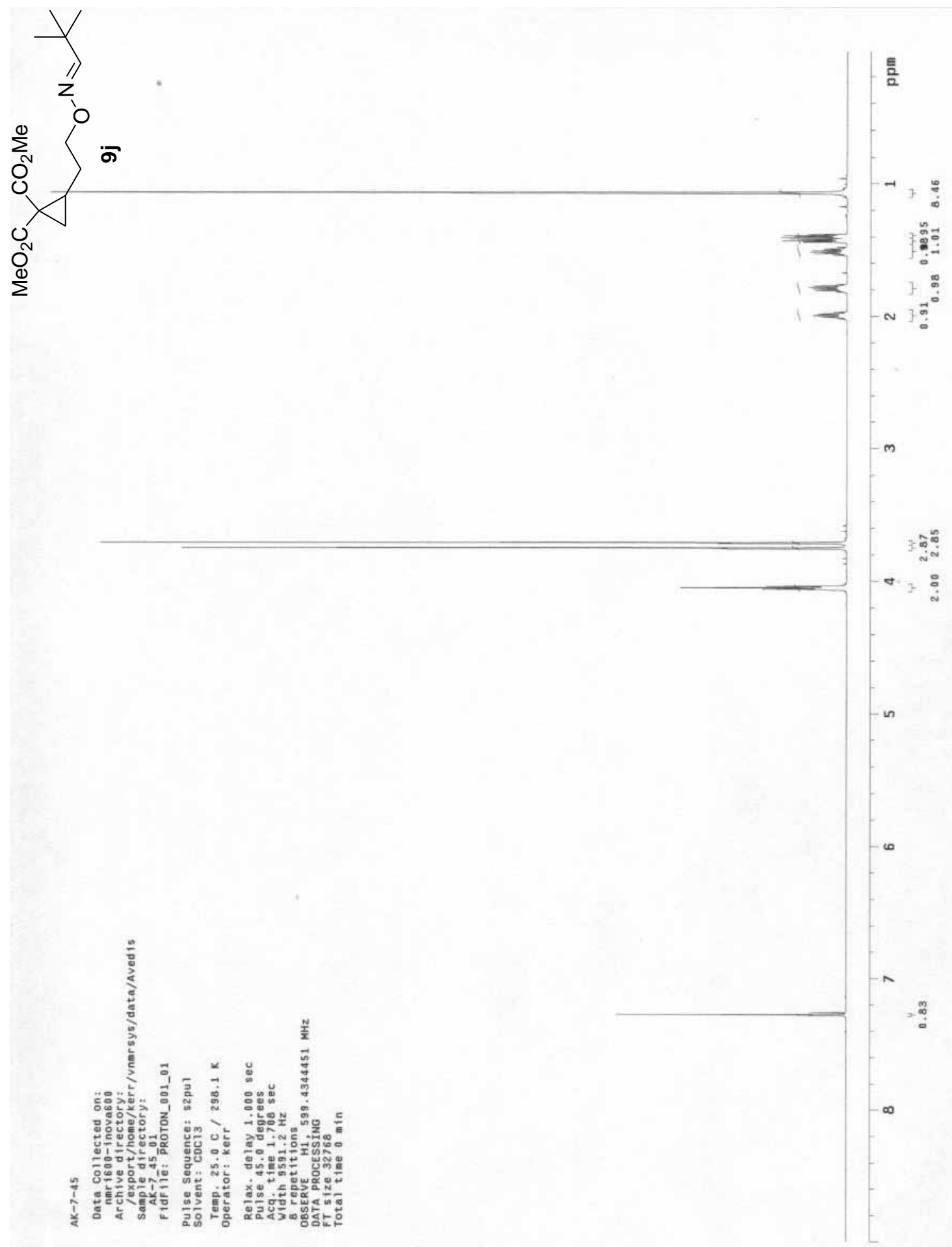




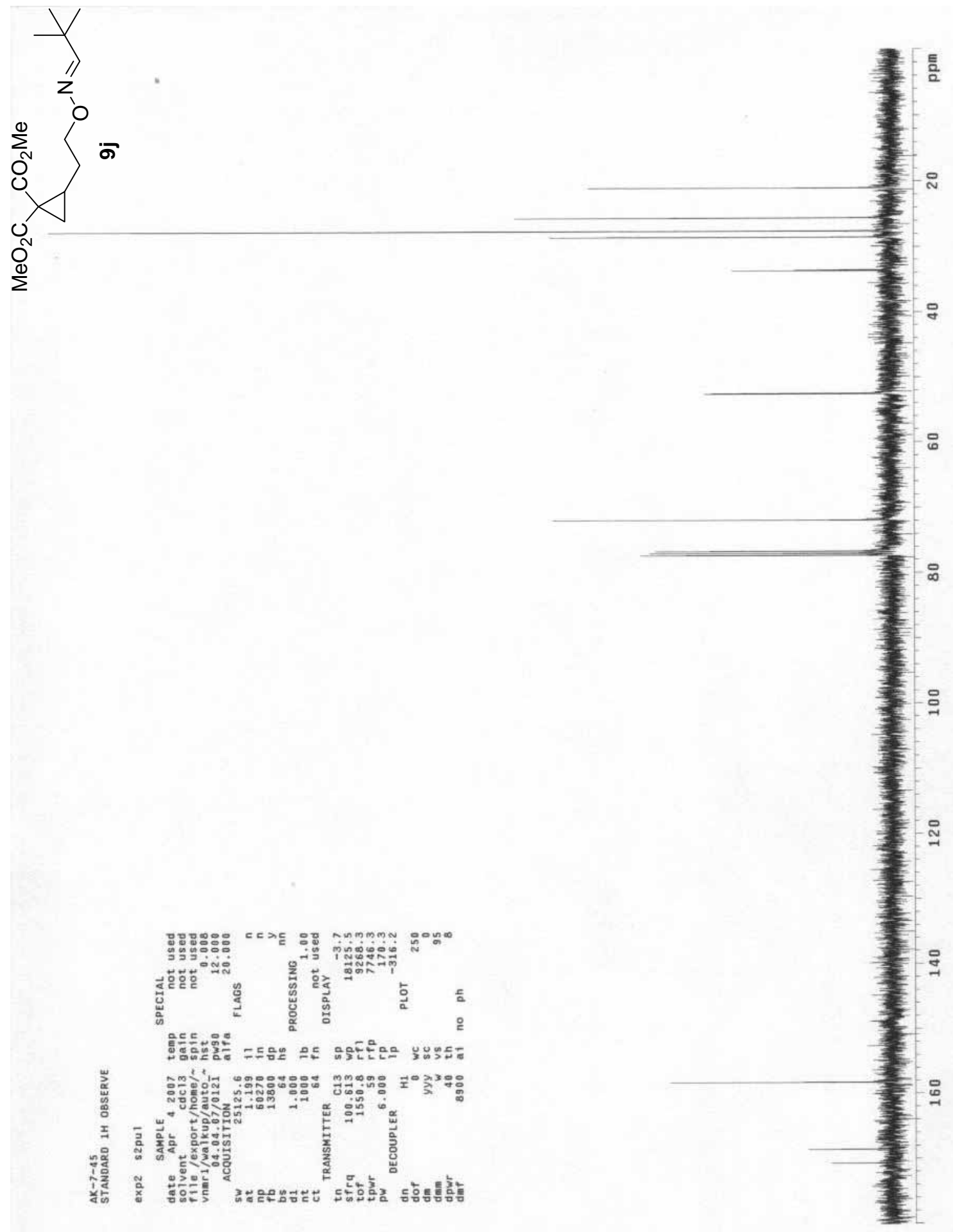




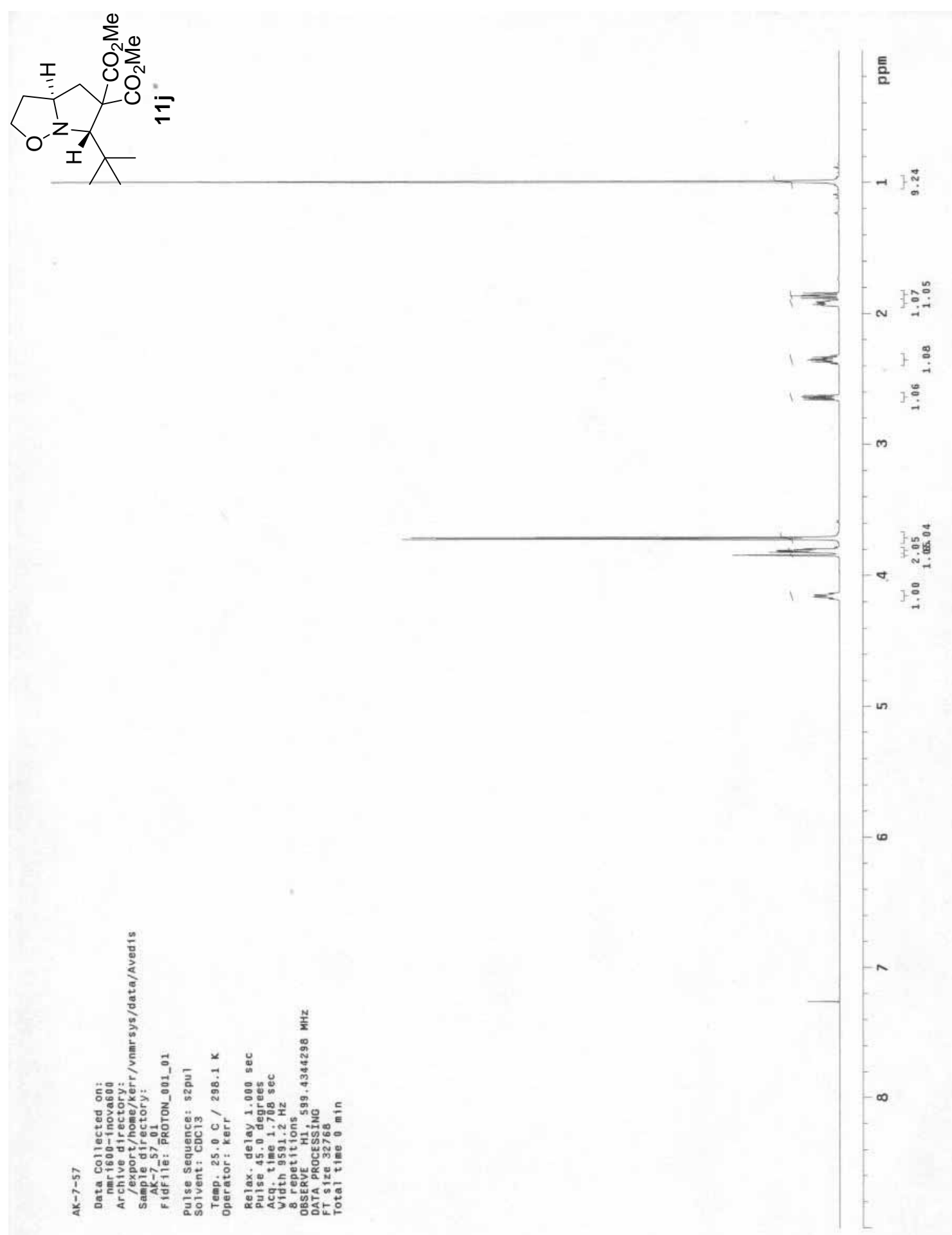



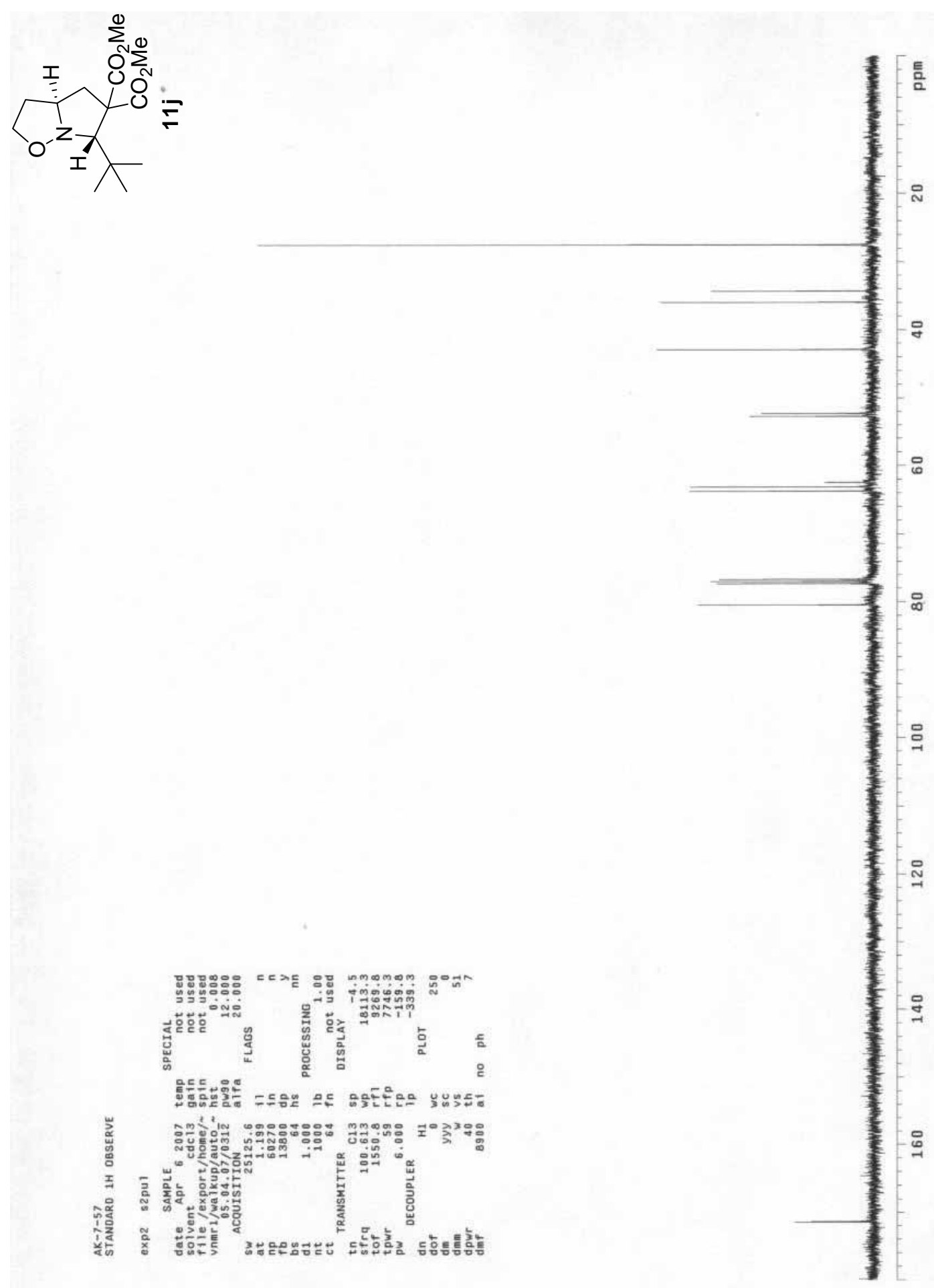


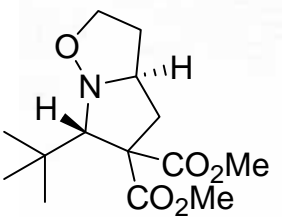

$(+I-)-11 \mathrm{j} ;(+)-\mathrm{Eu}(\mathrm{hfc})_{3}$

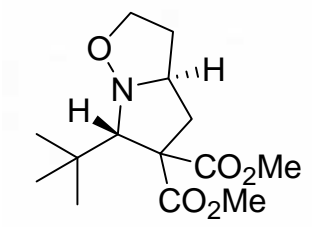

$(2 S, 5 R)-11 \mathrm{j} ;(+)-\mathrm{Eu}(\mathrm{hfc})_{3}$
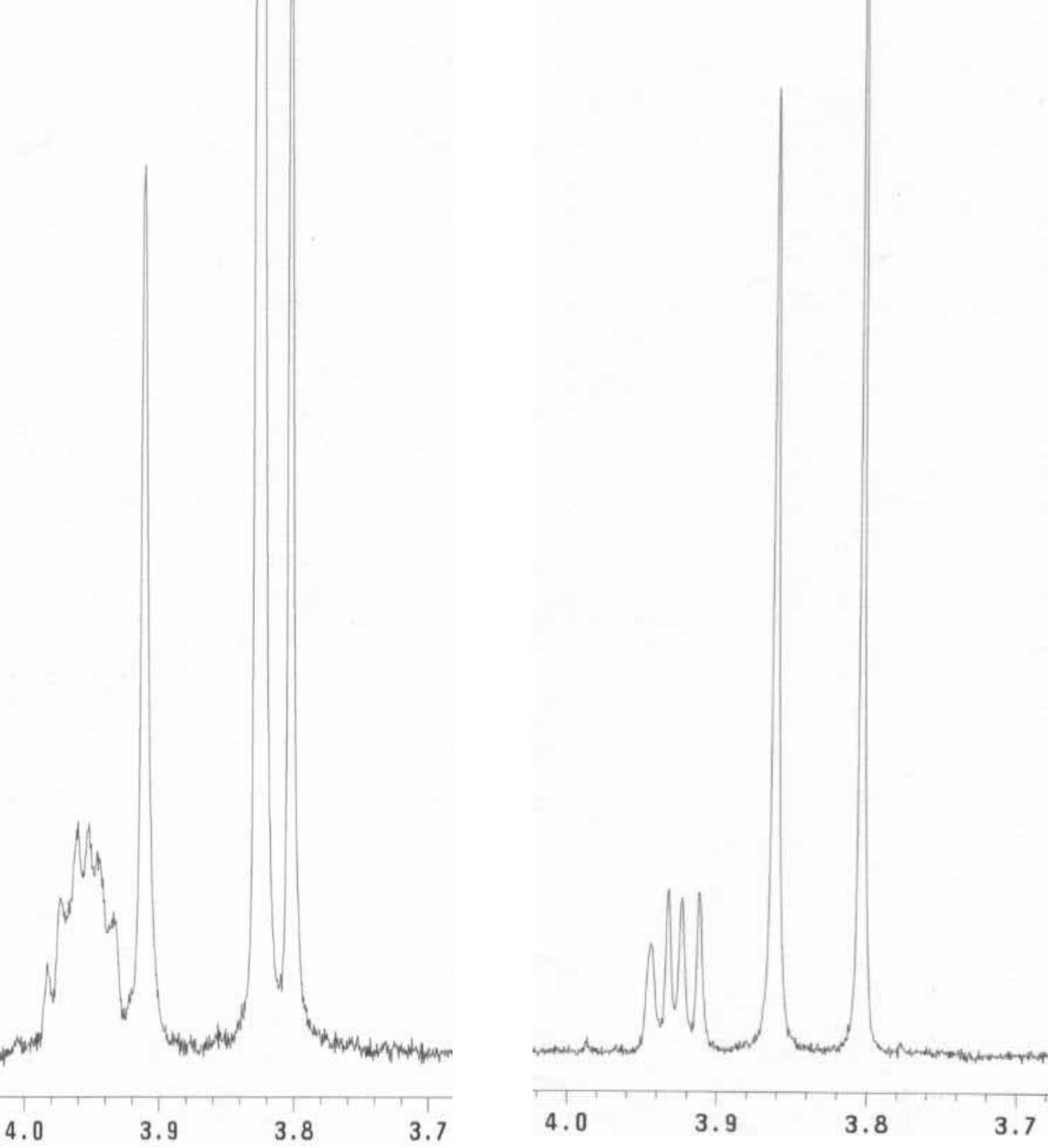

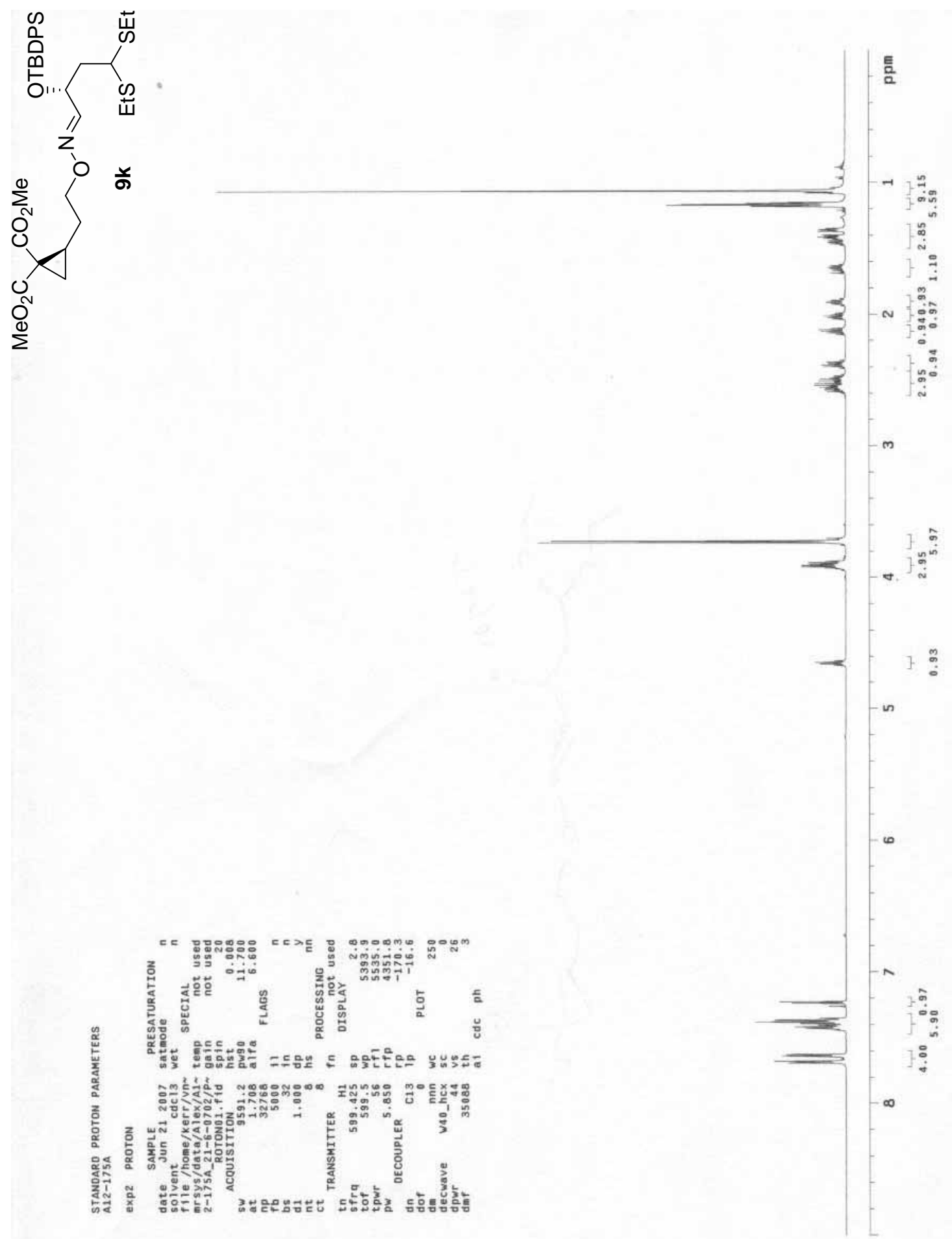

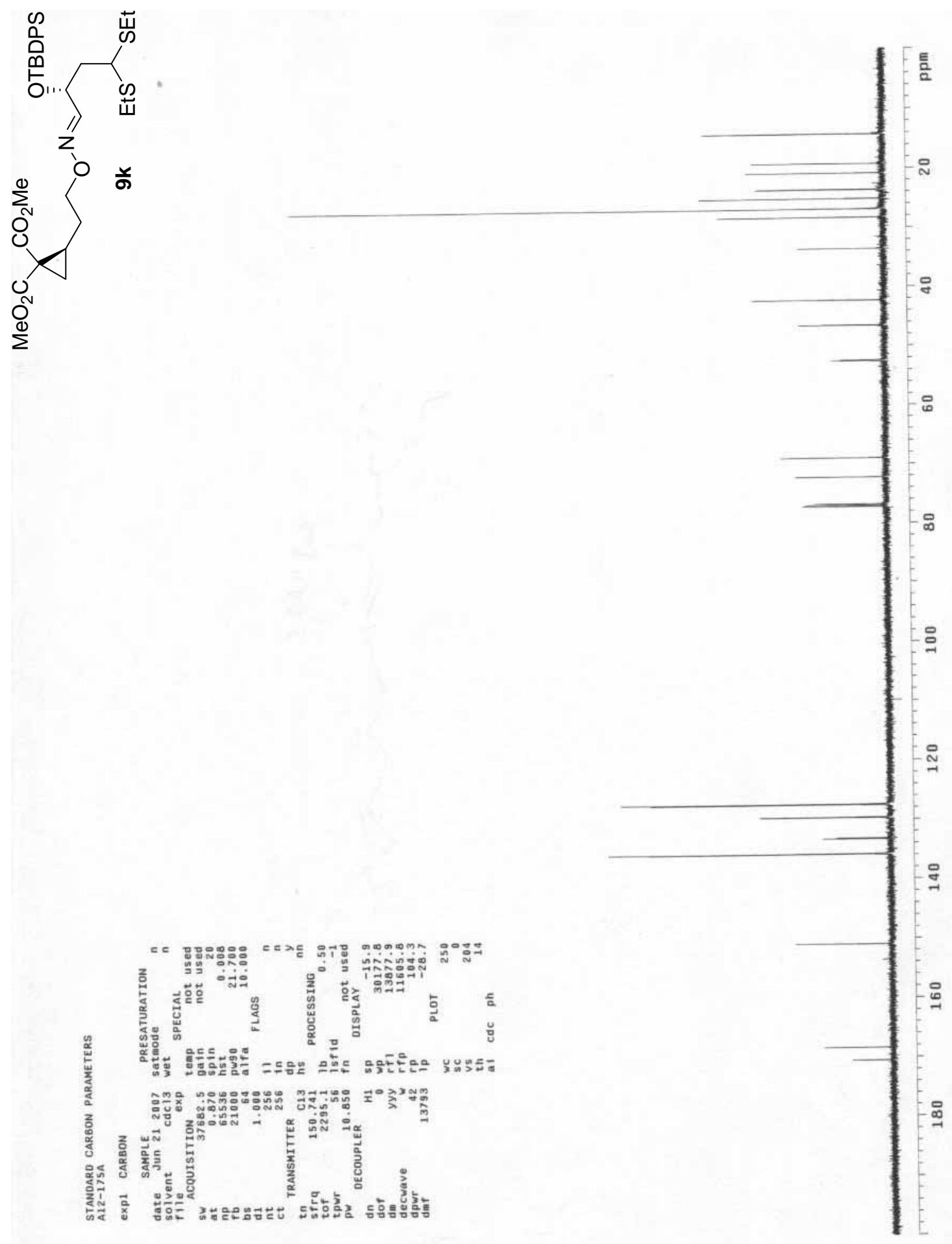


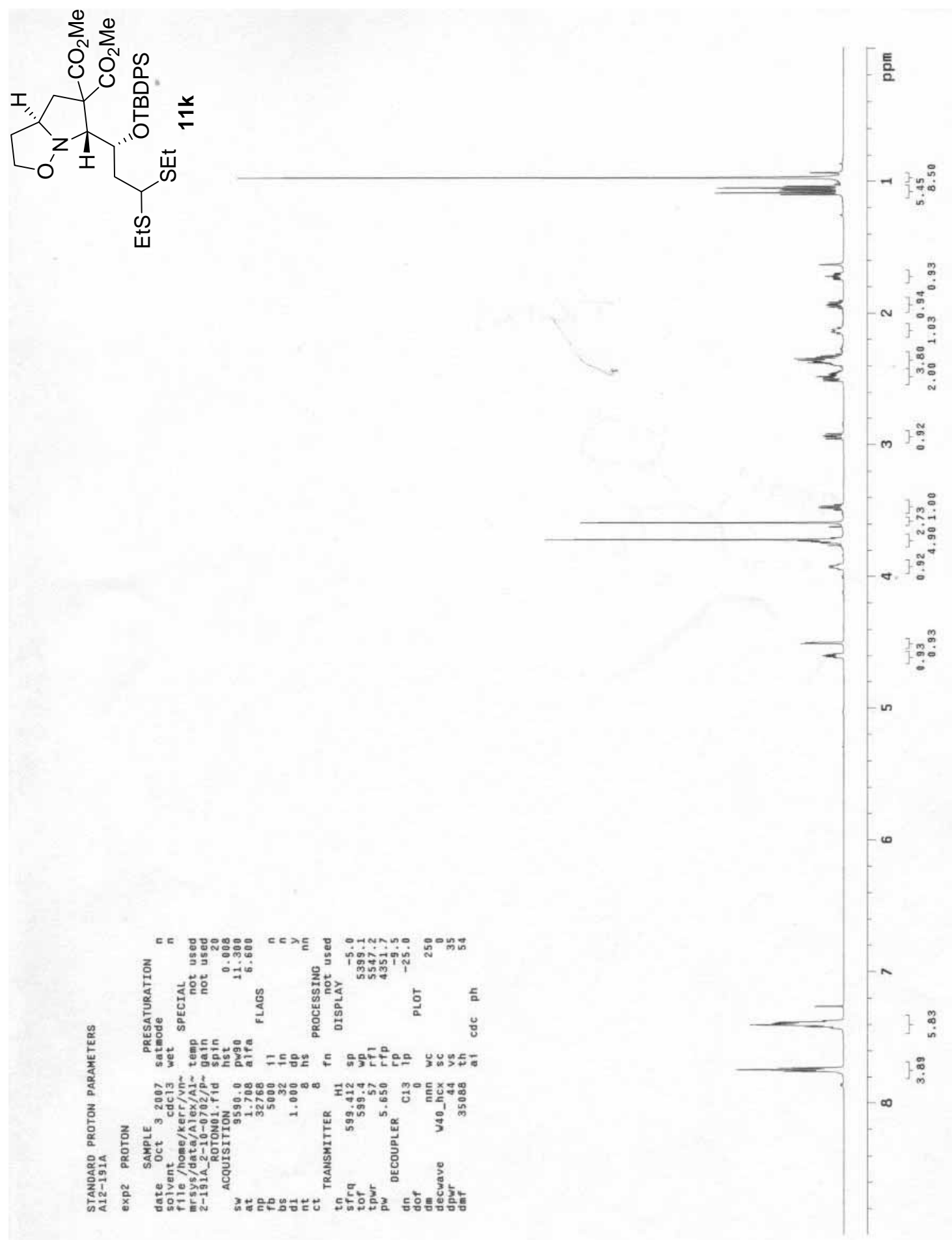



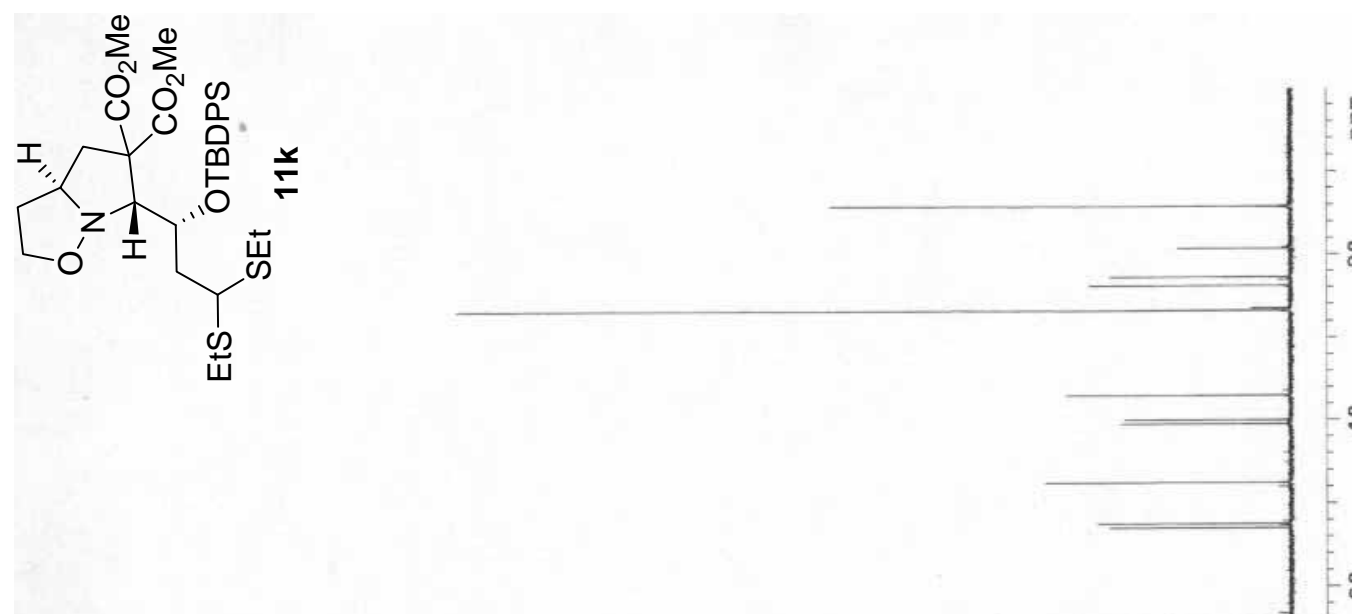

흠

0

$\infty$

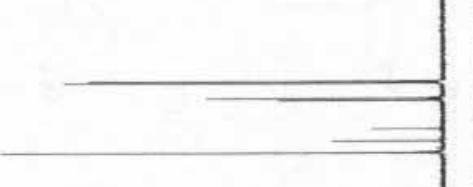

억

웅

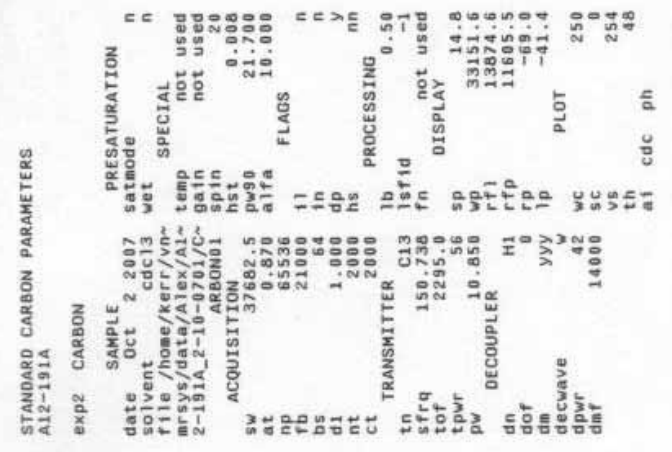




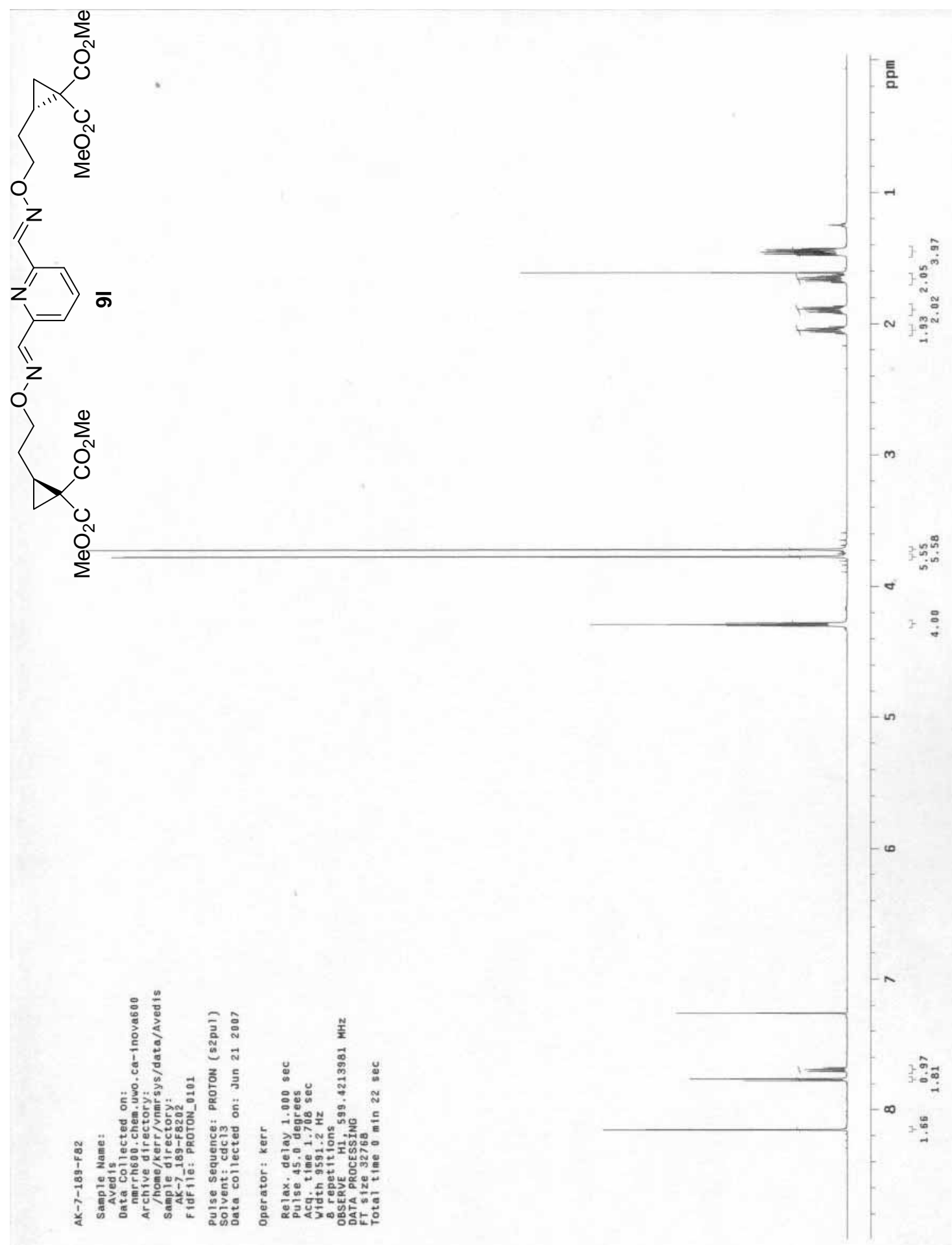



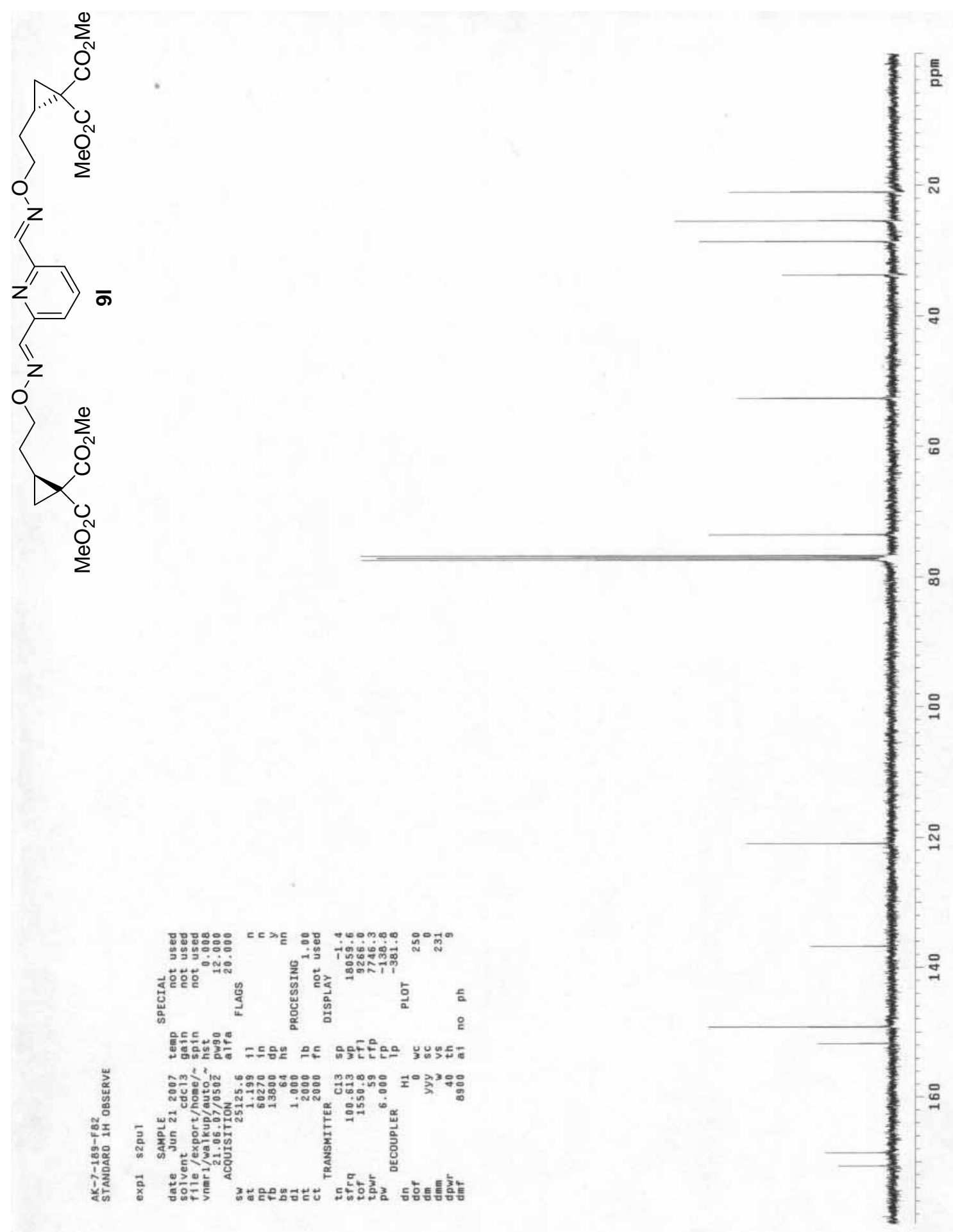


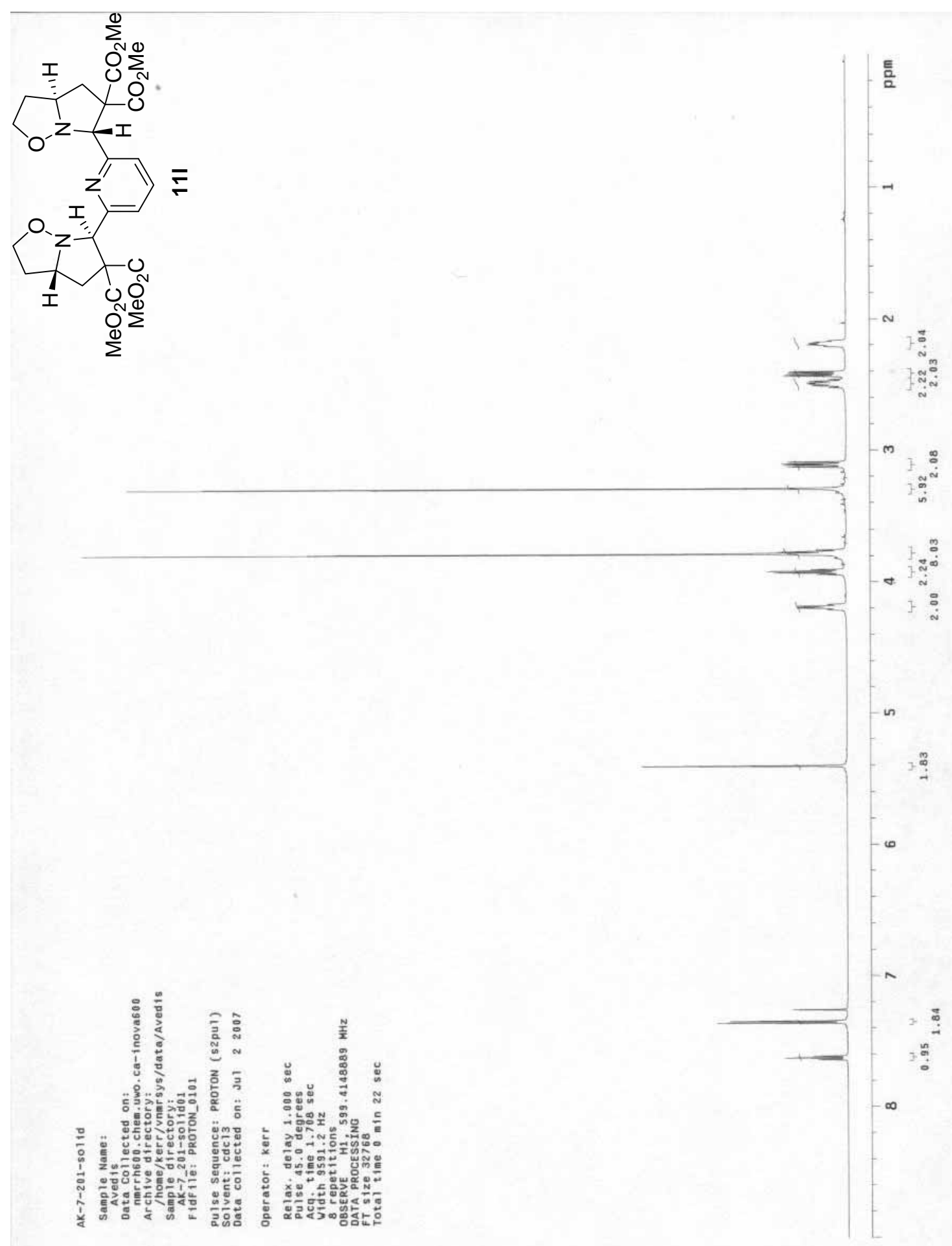



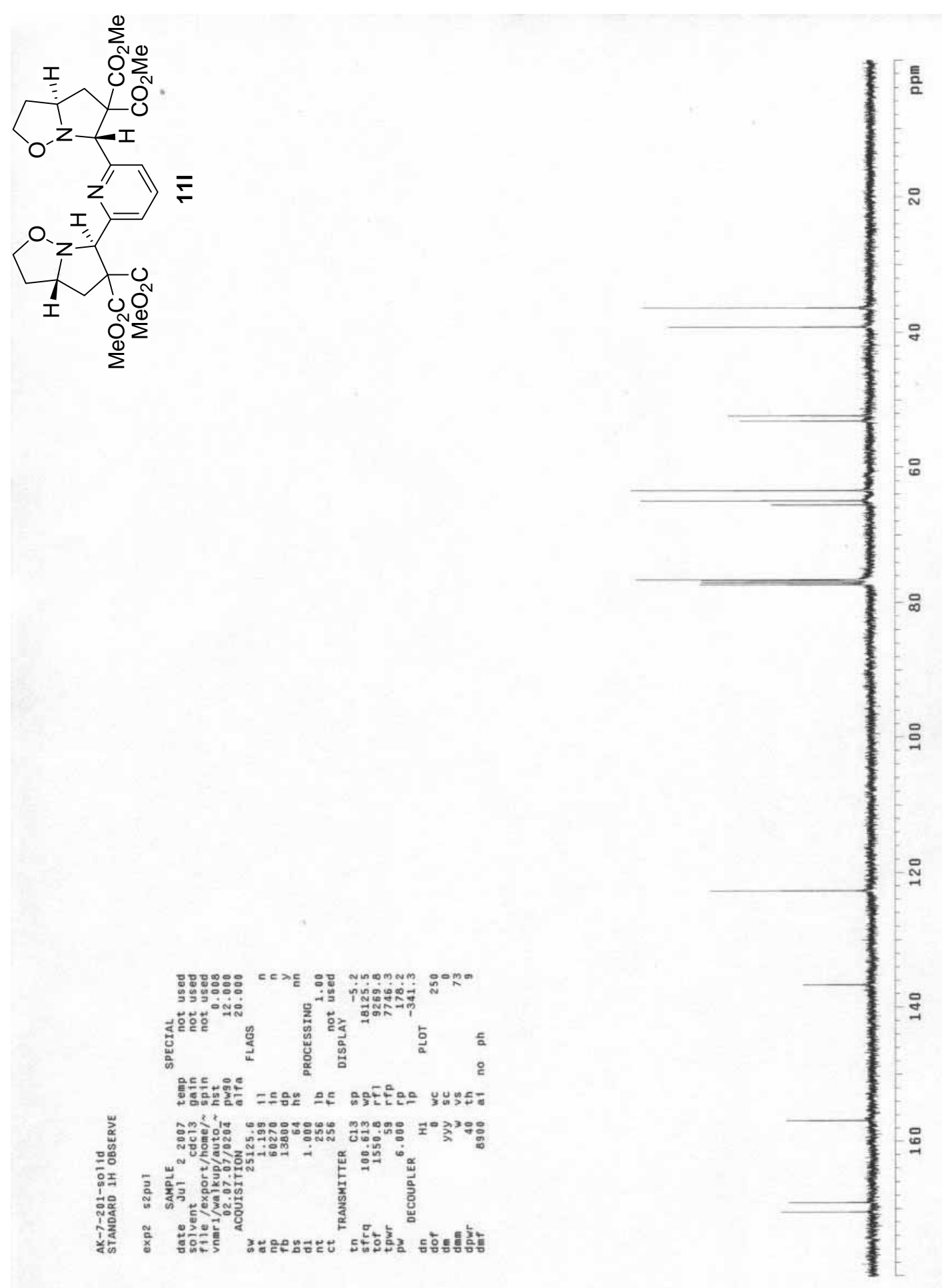

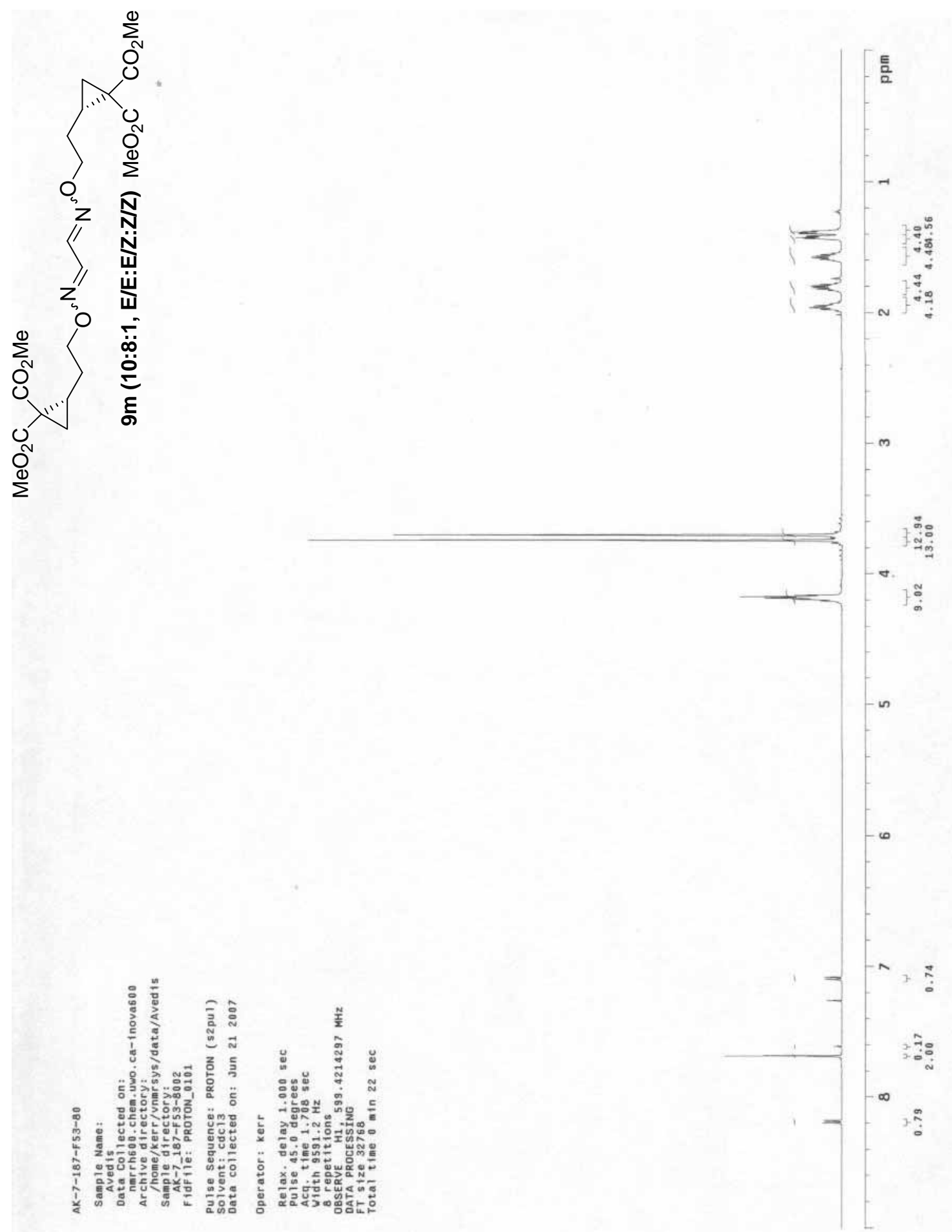

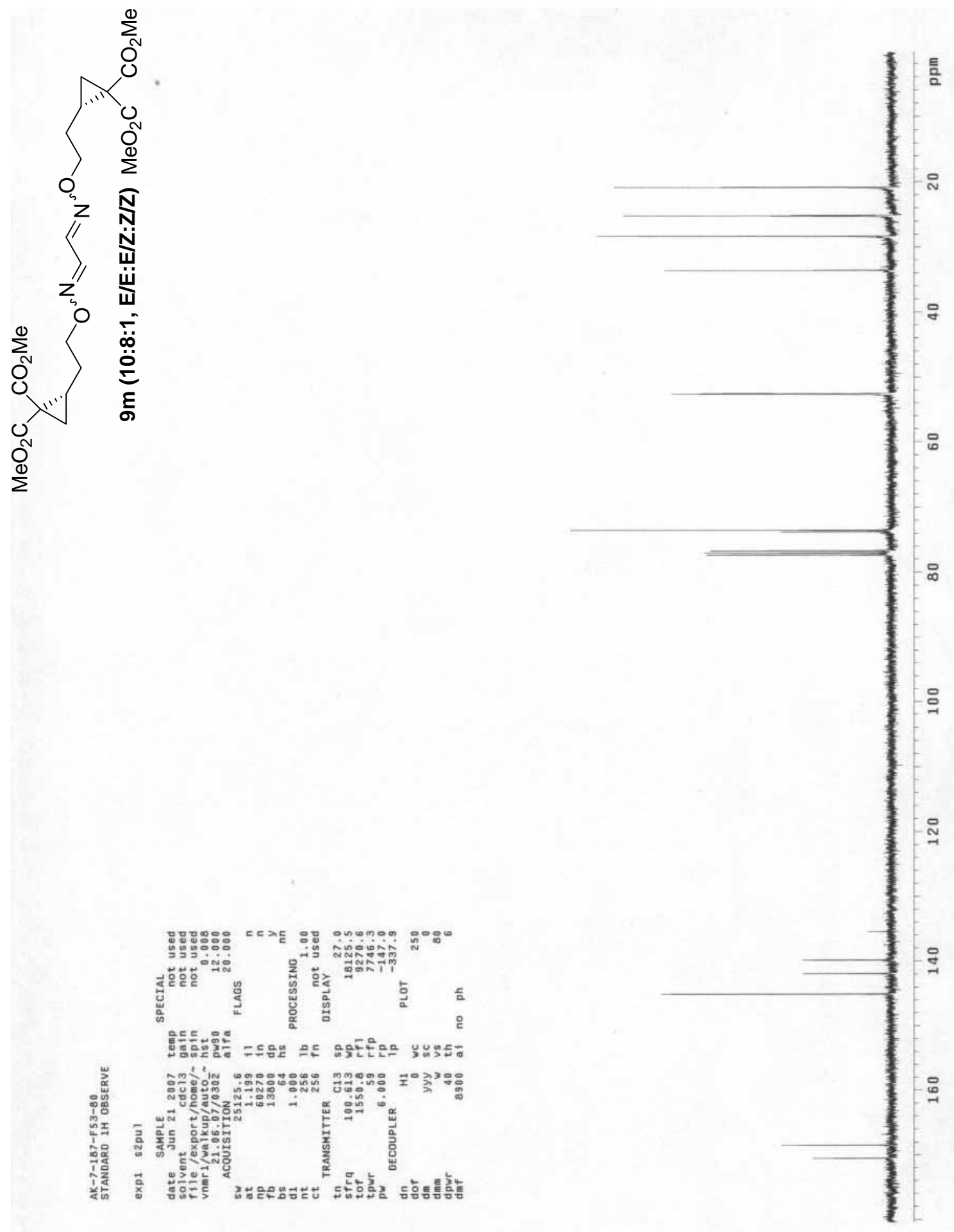

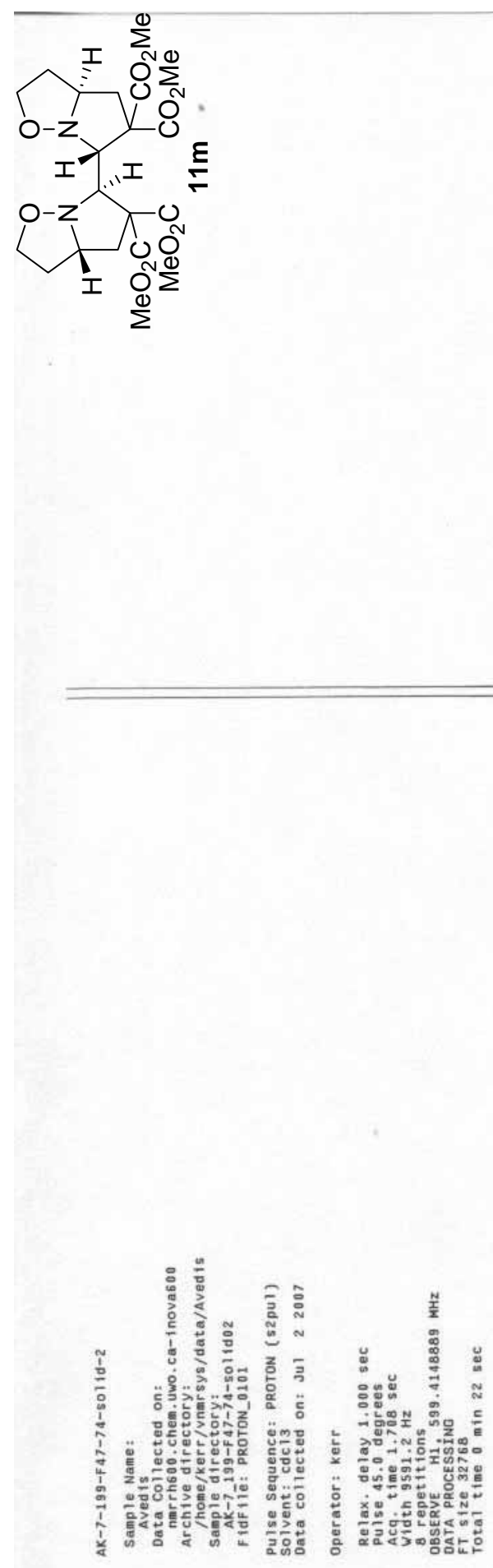

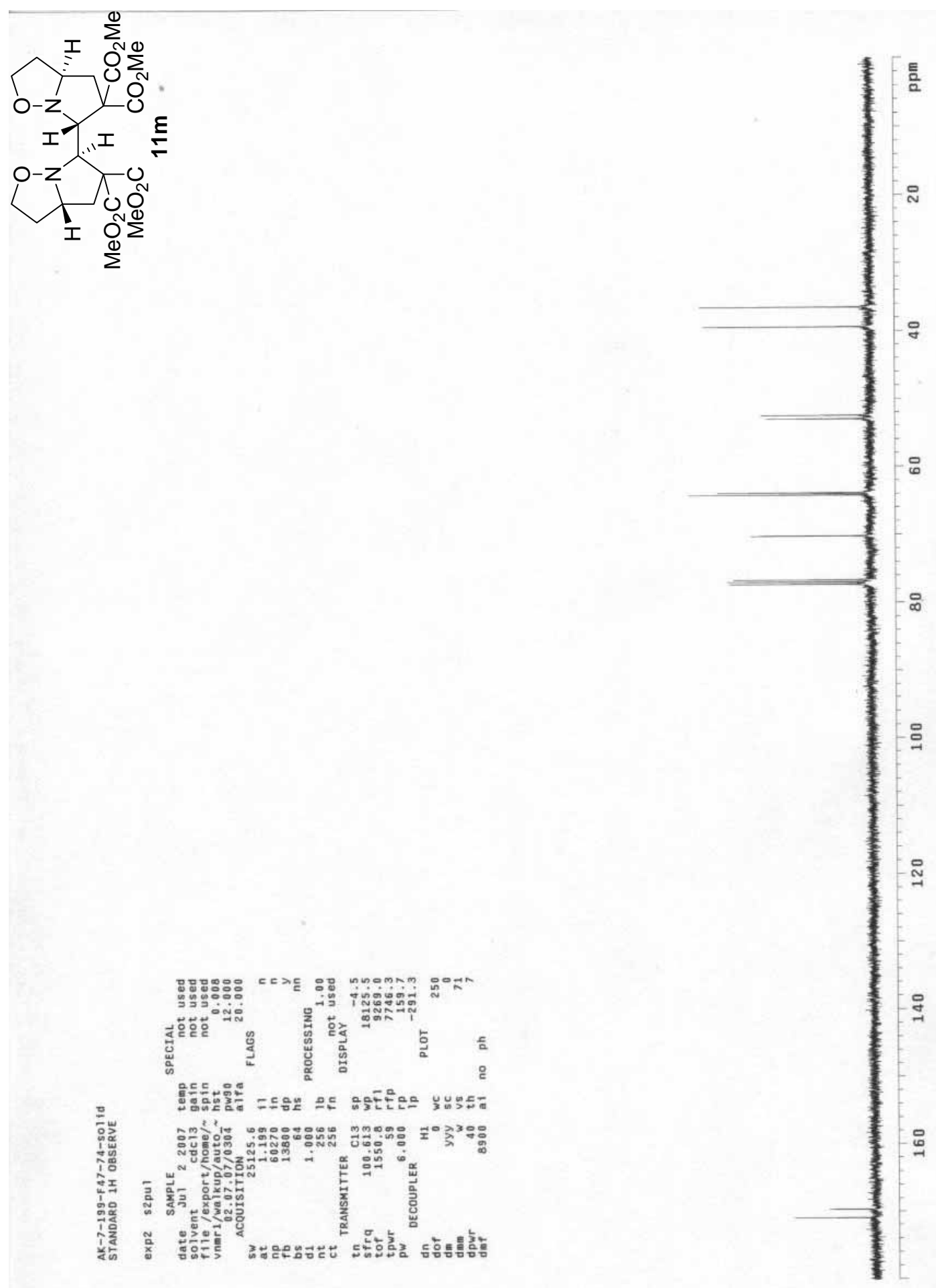

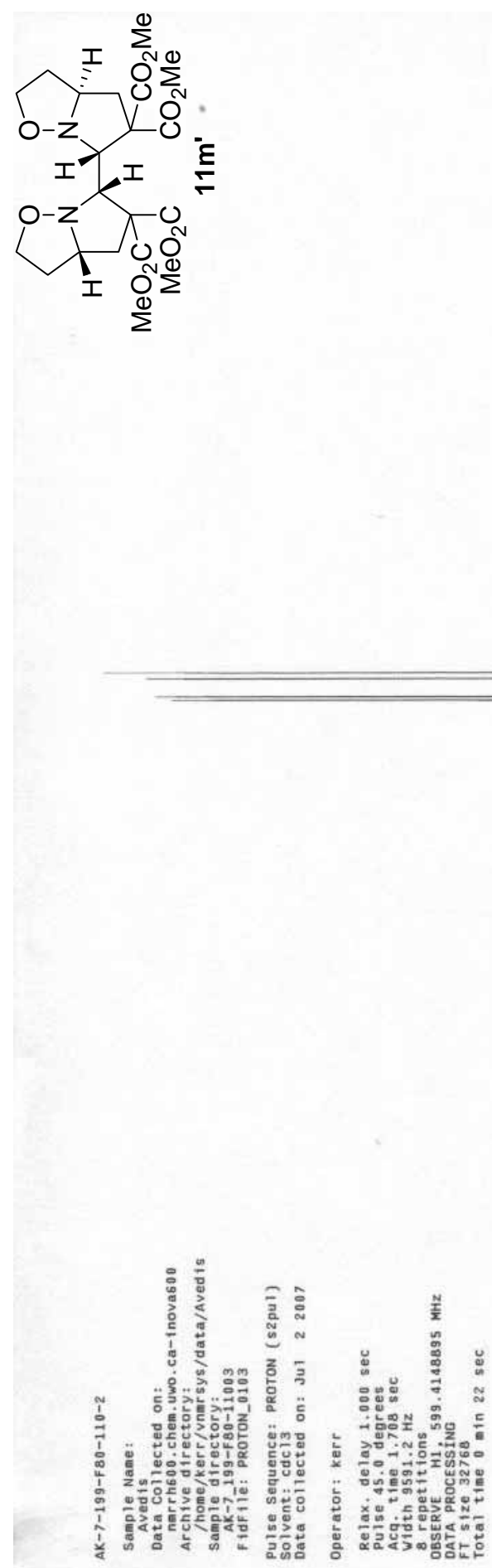

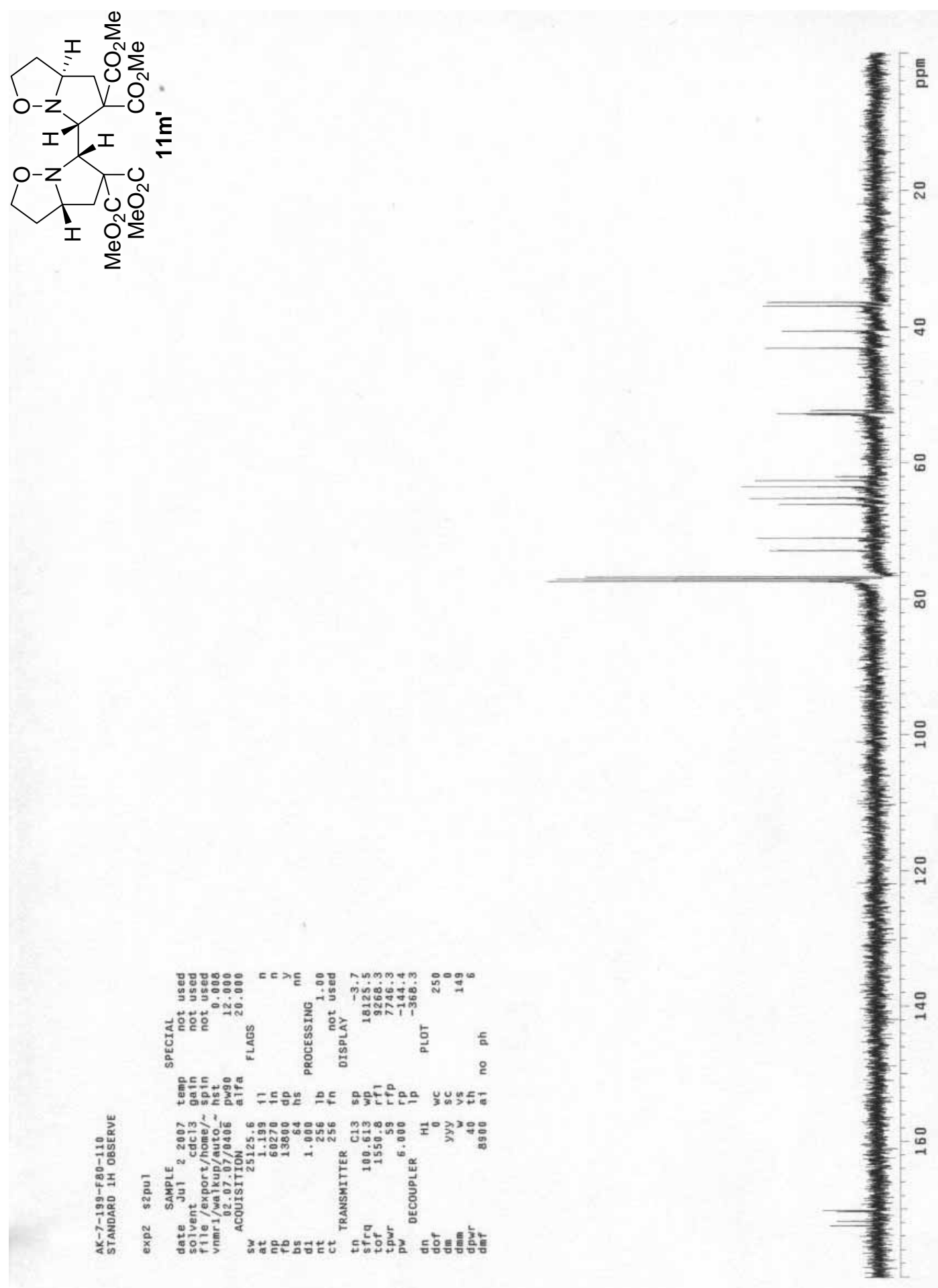

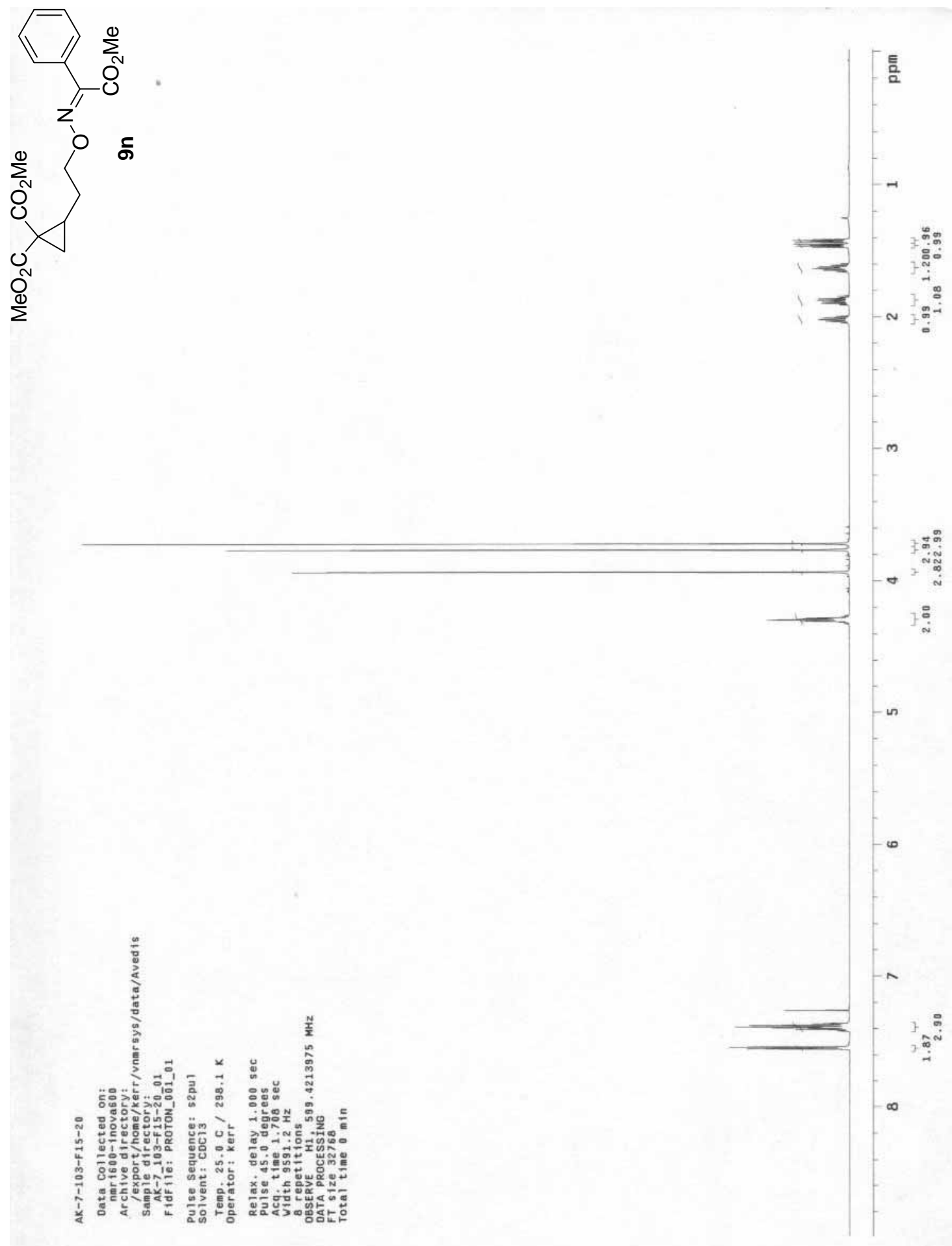

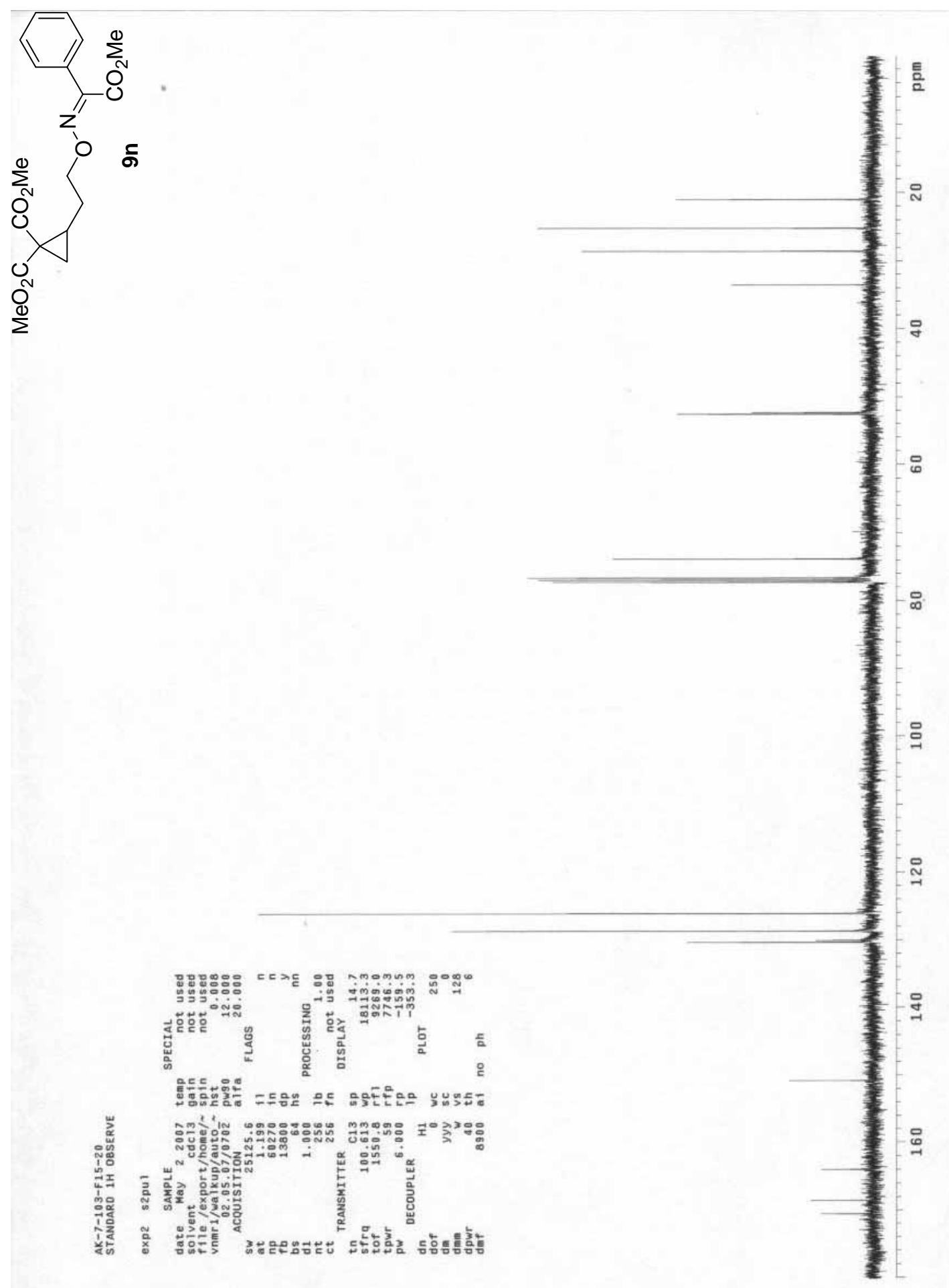


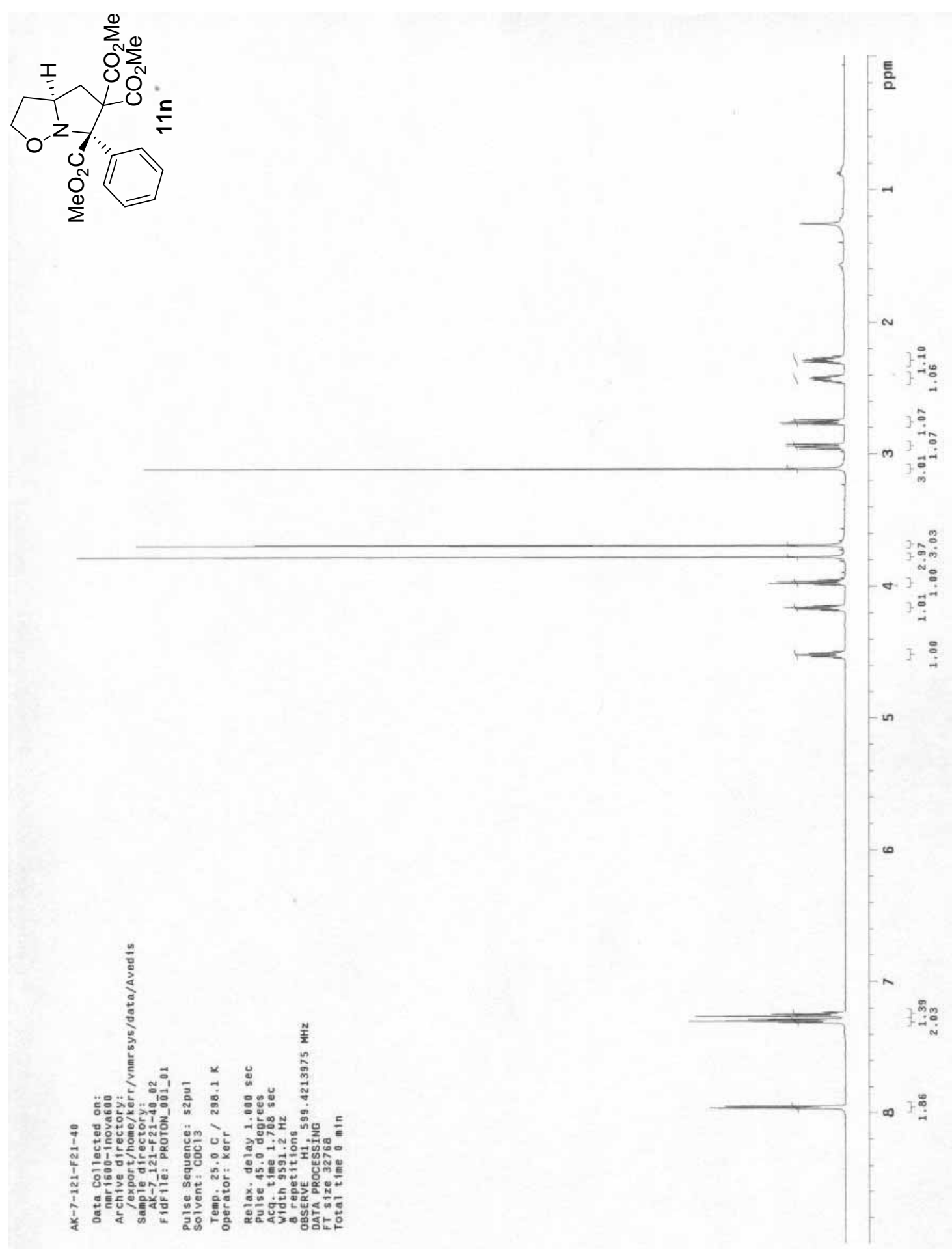



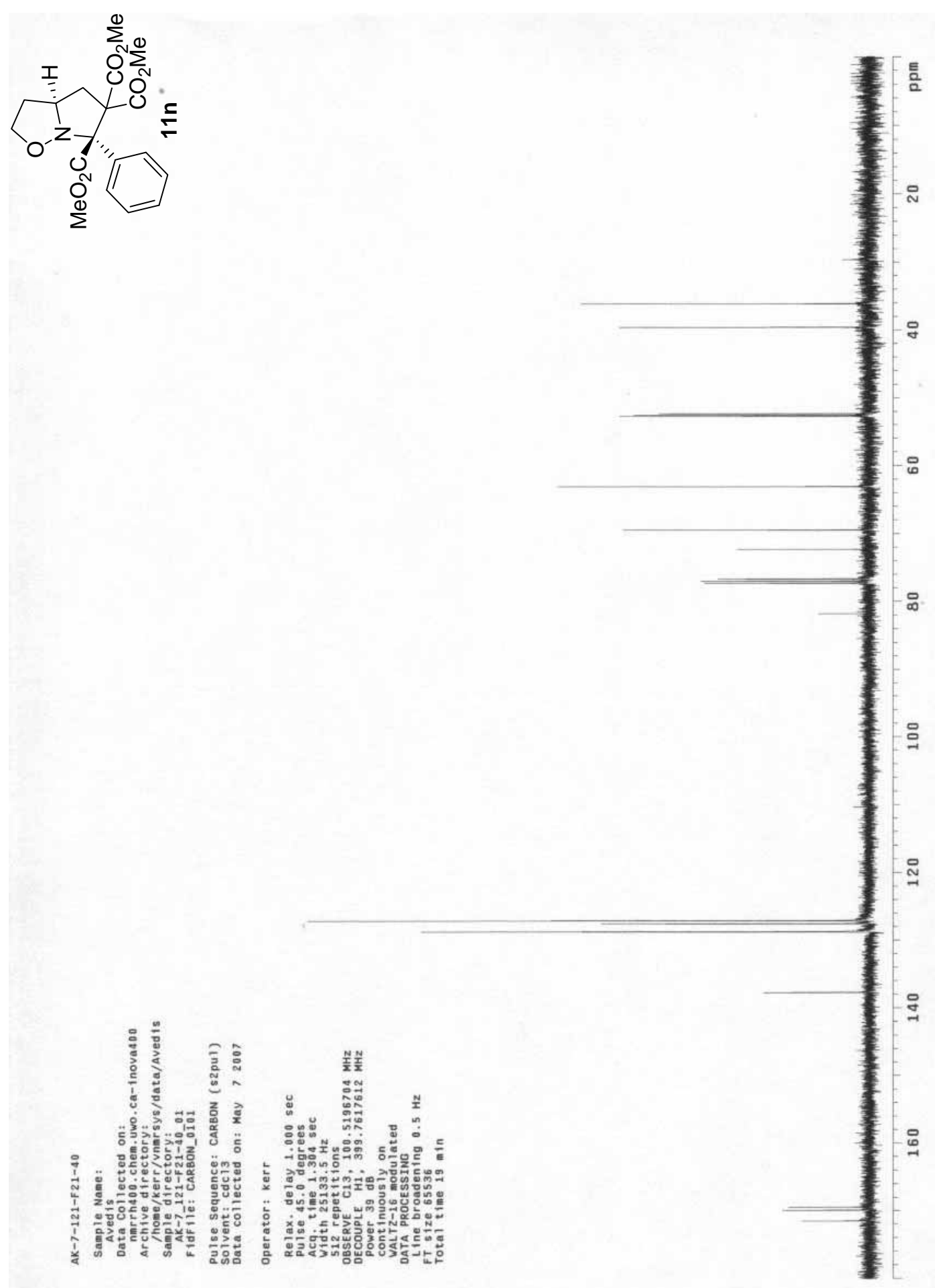


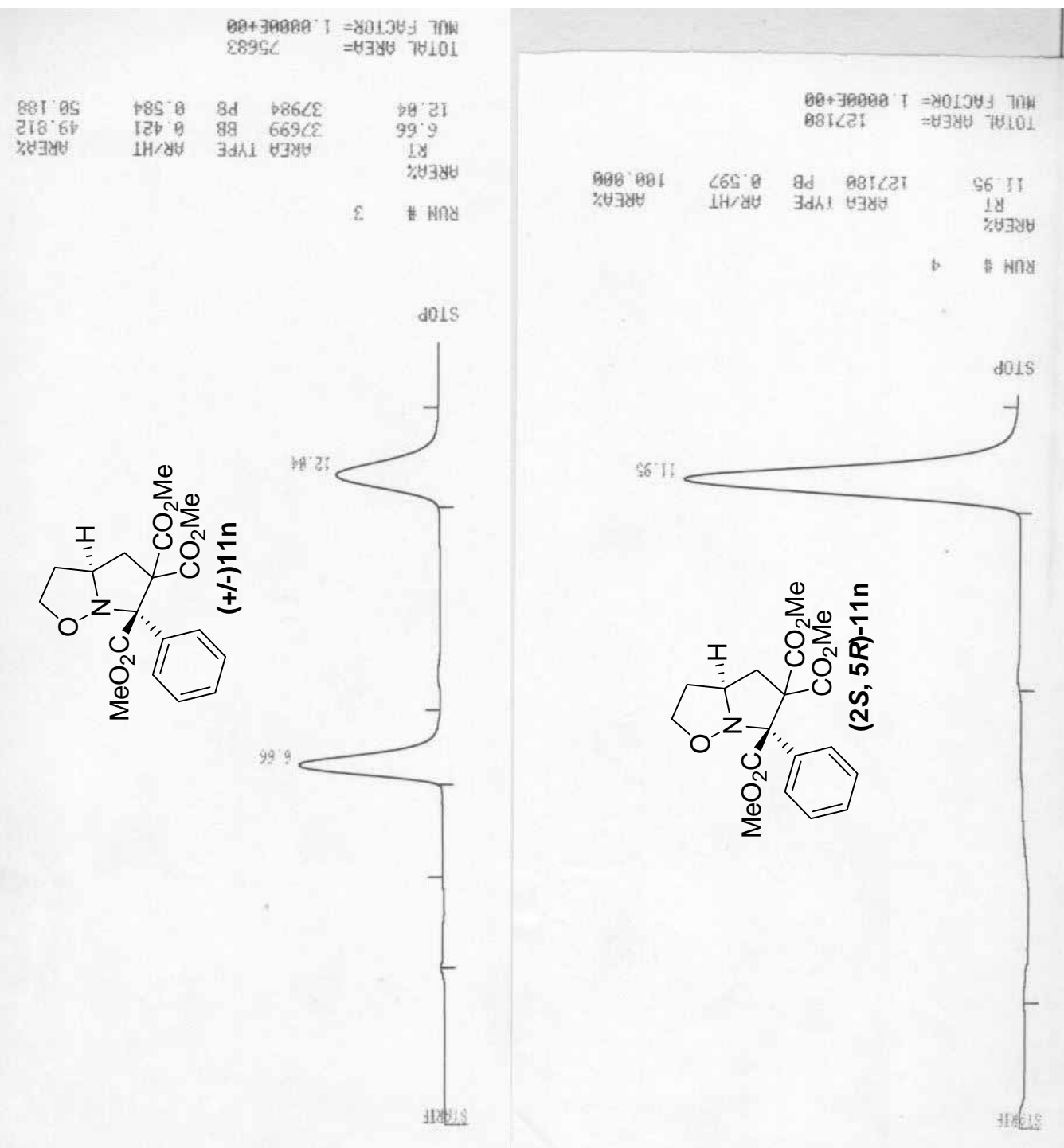




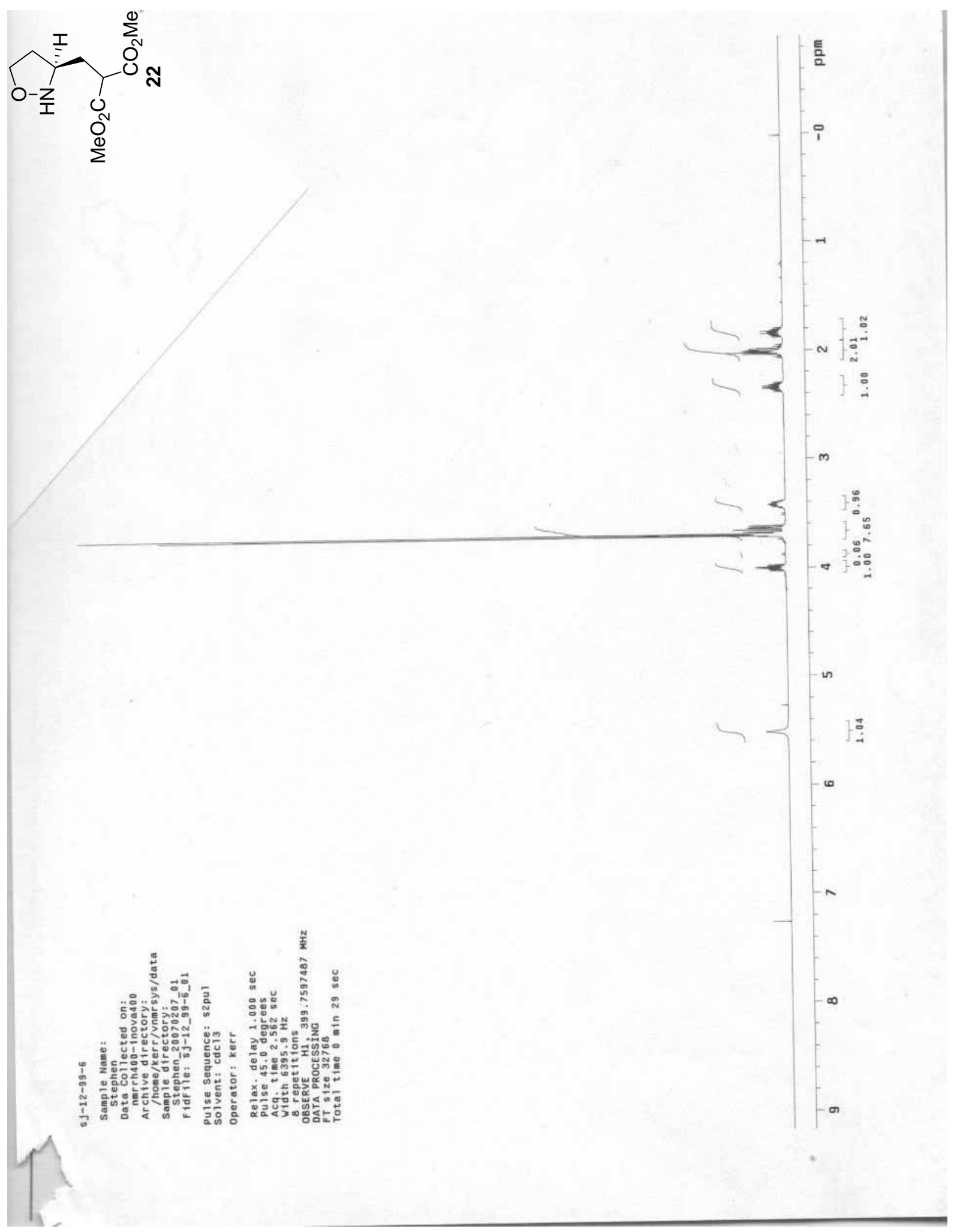



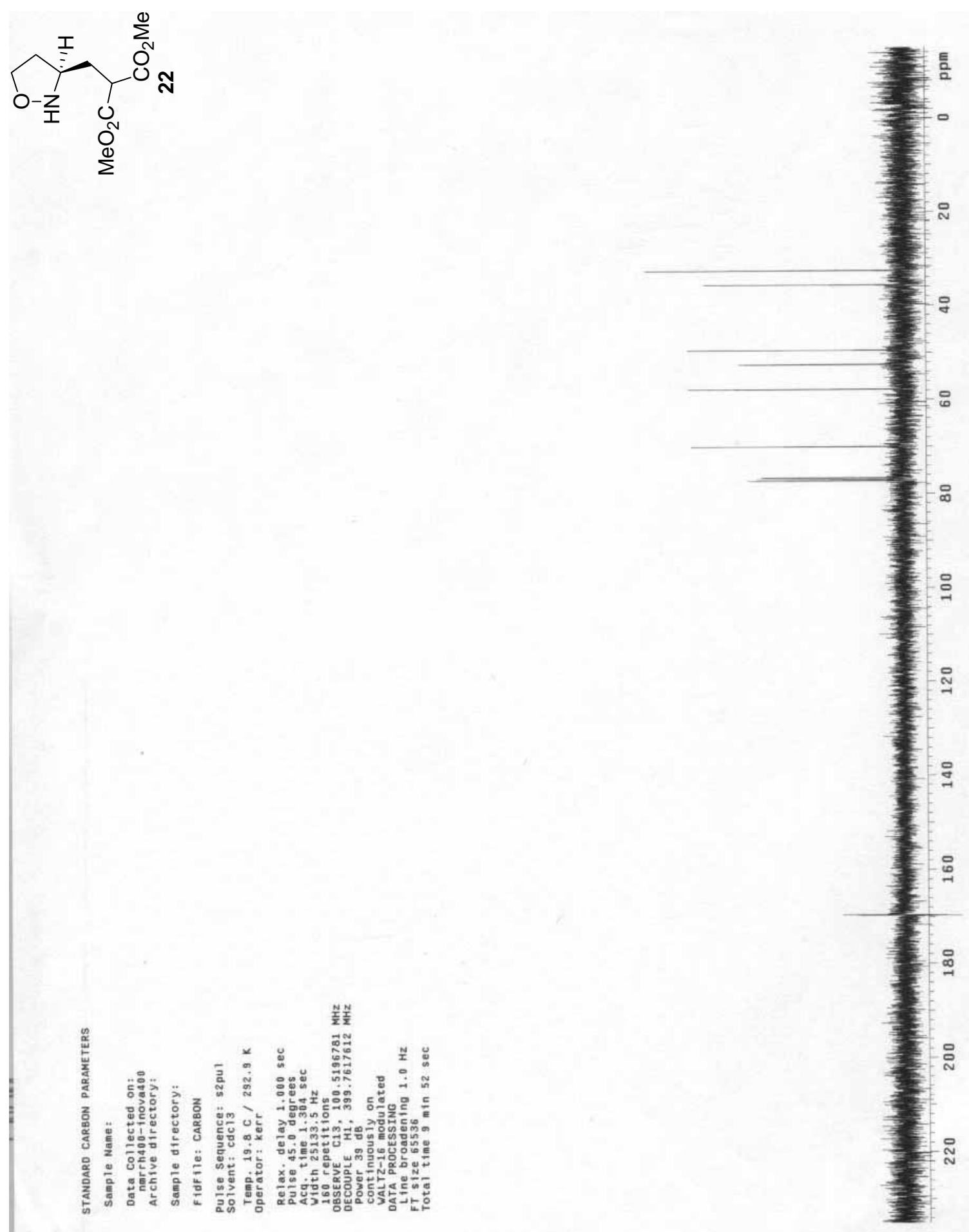

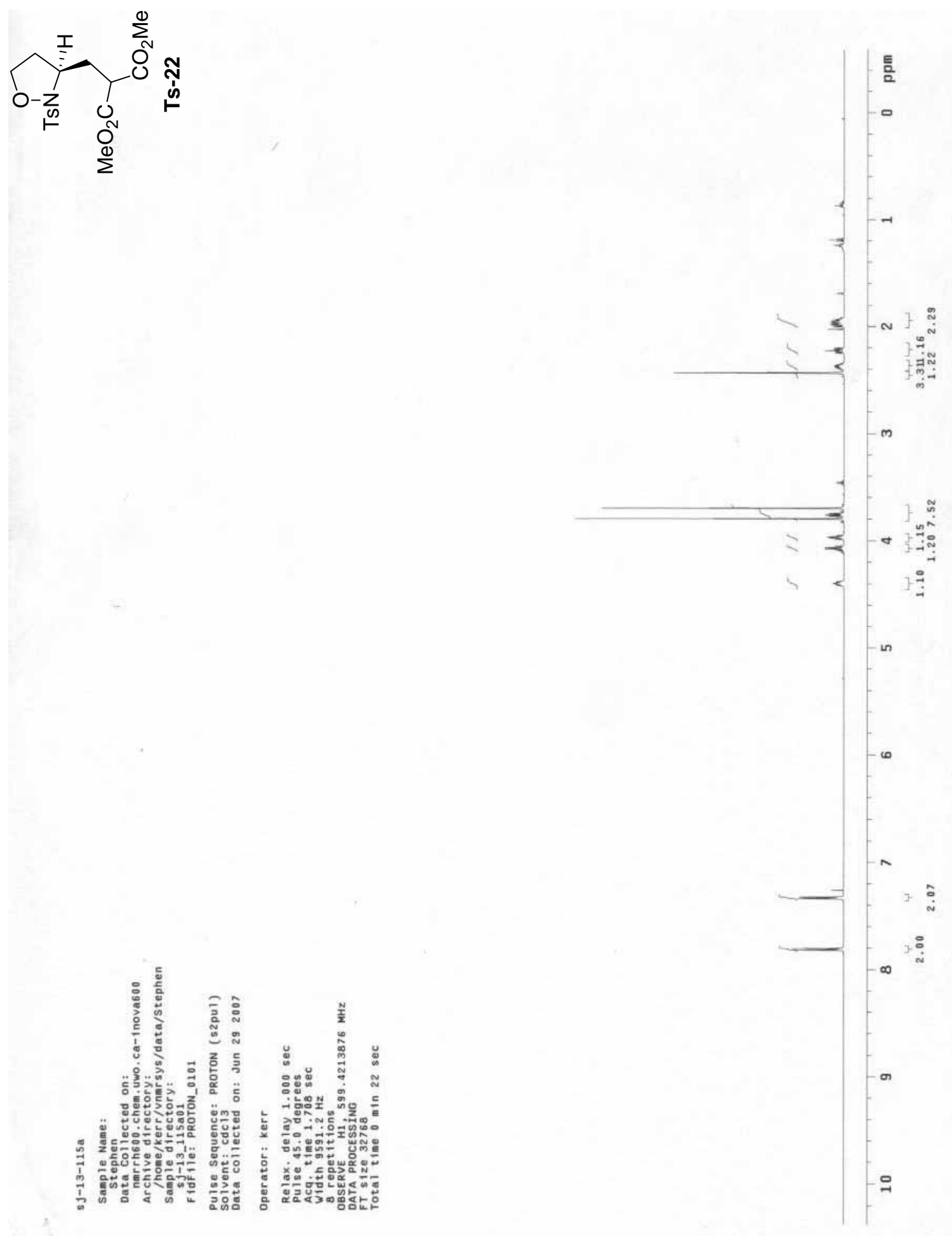

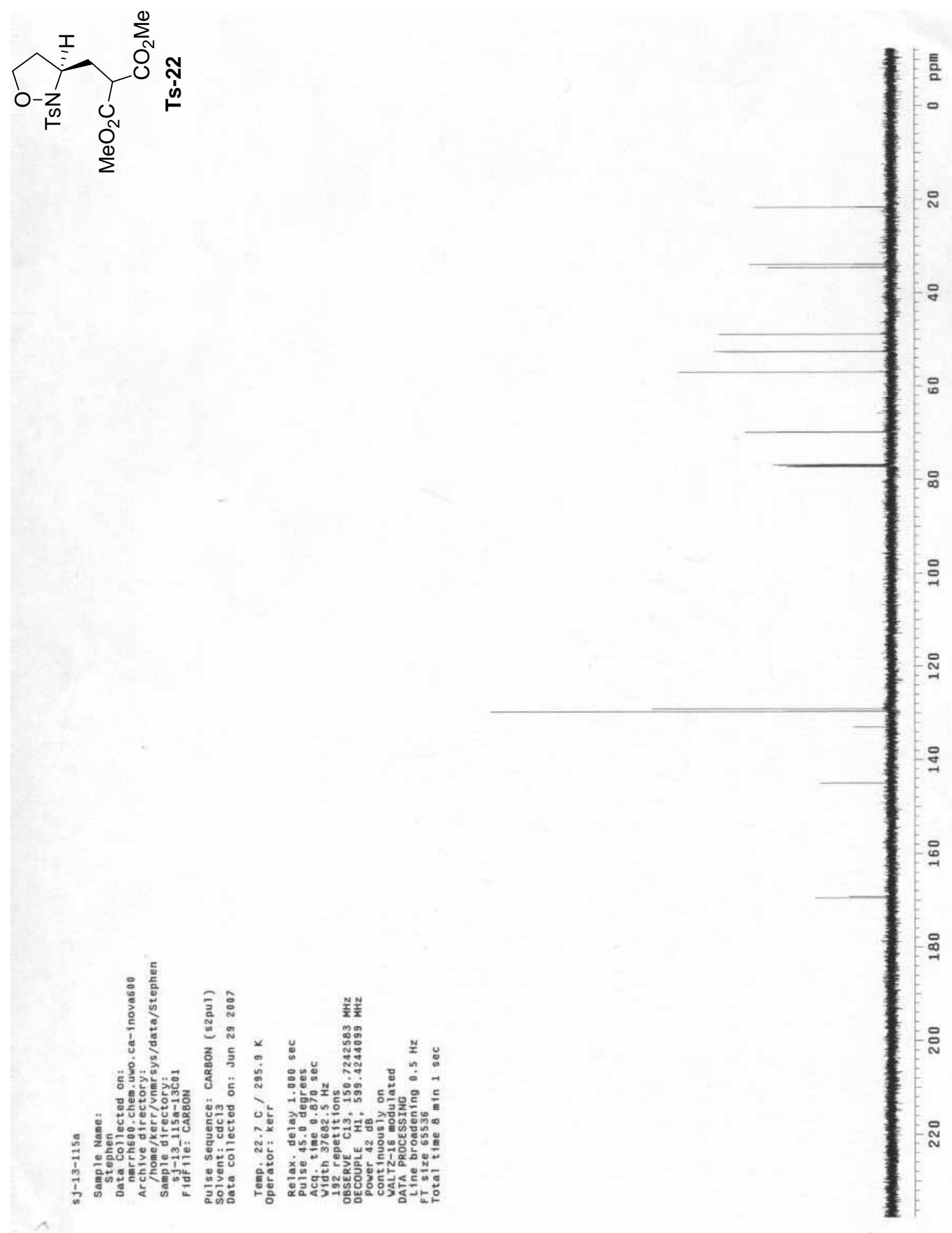


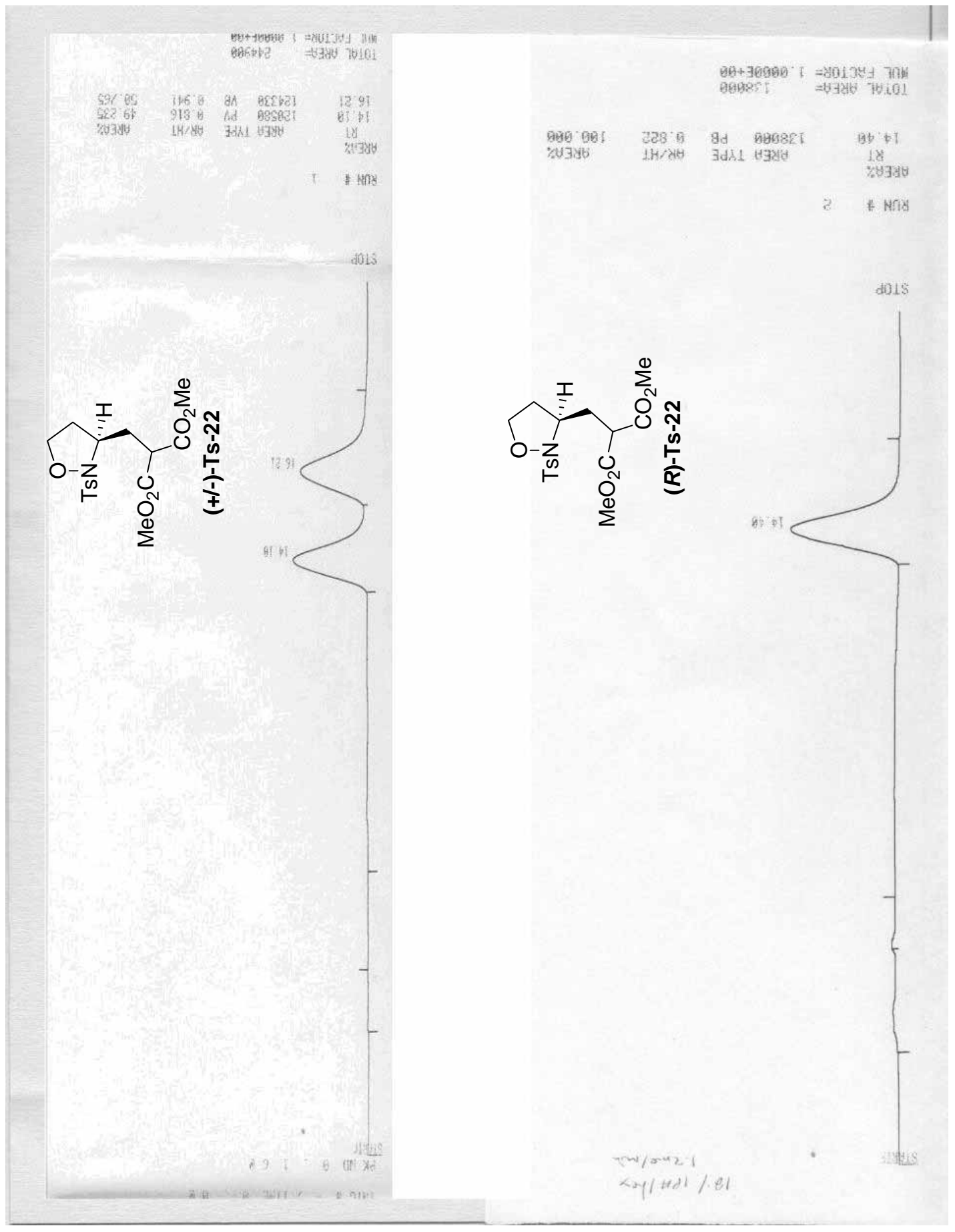




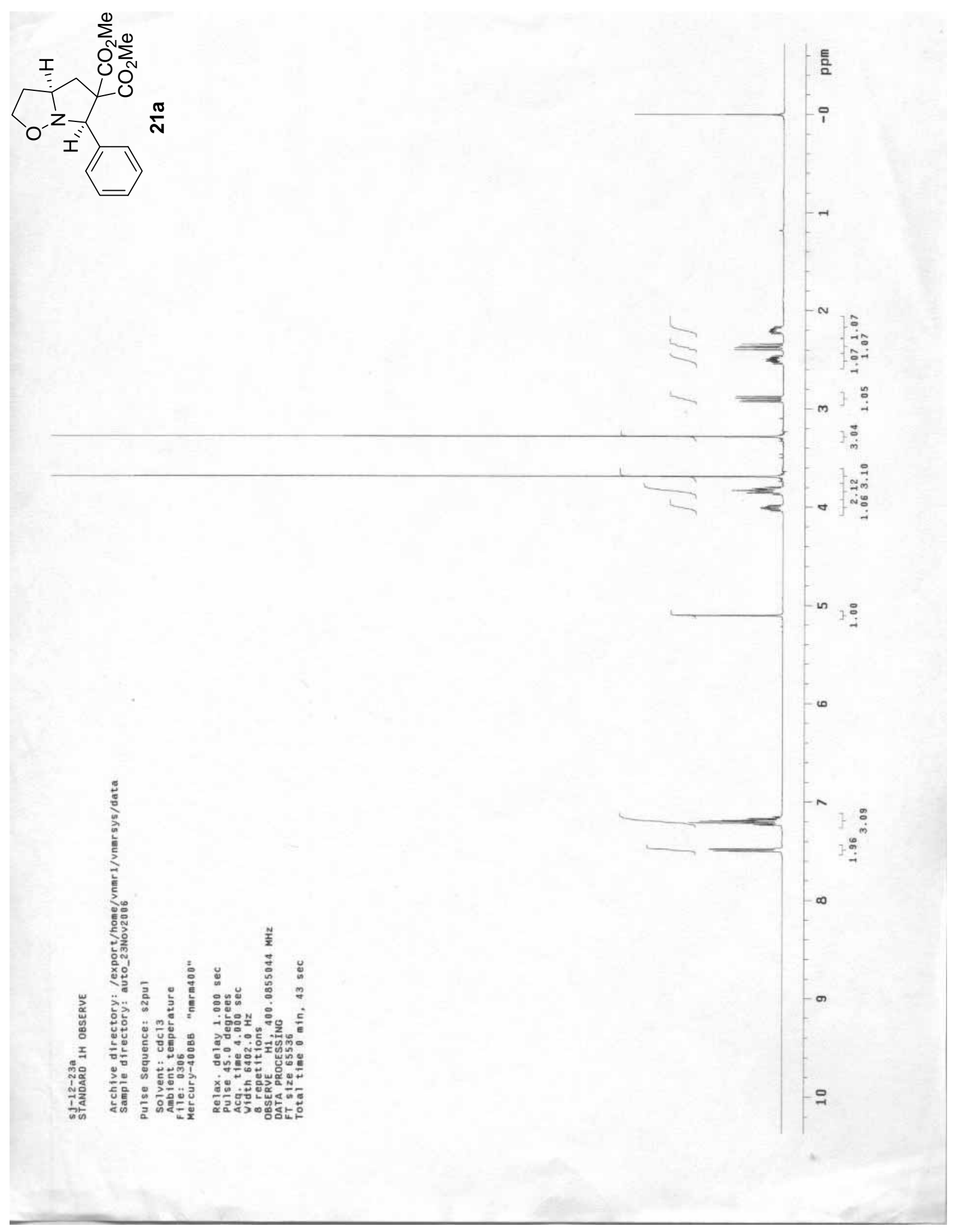




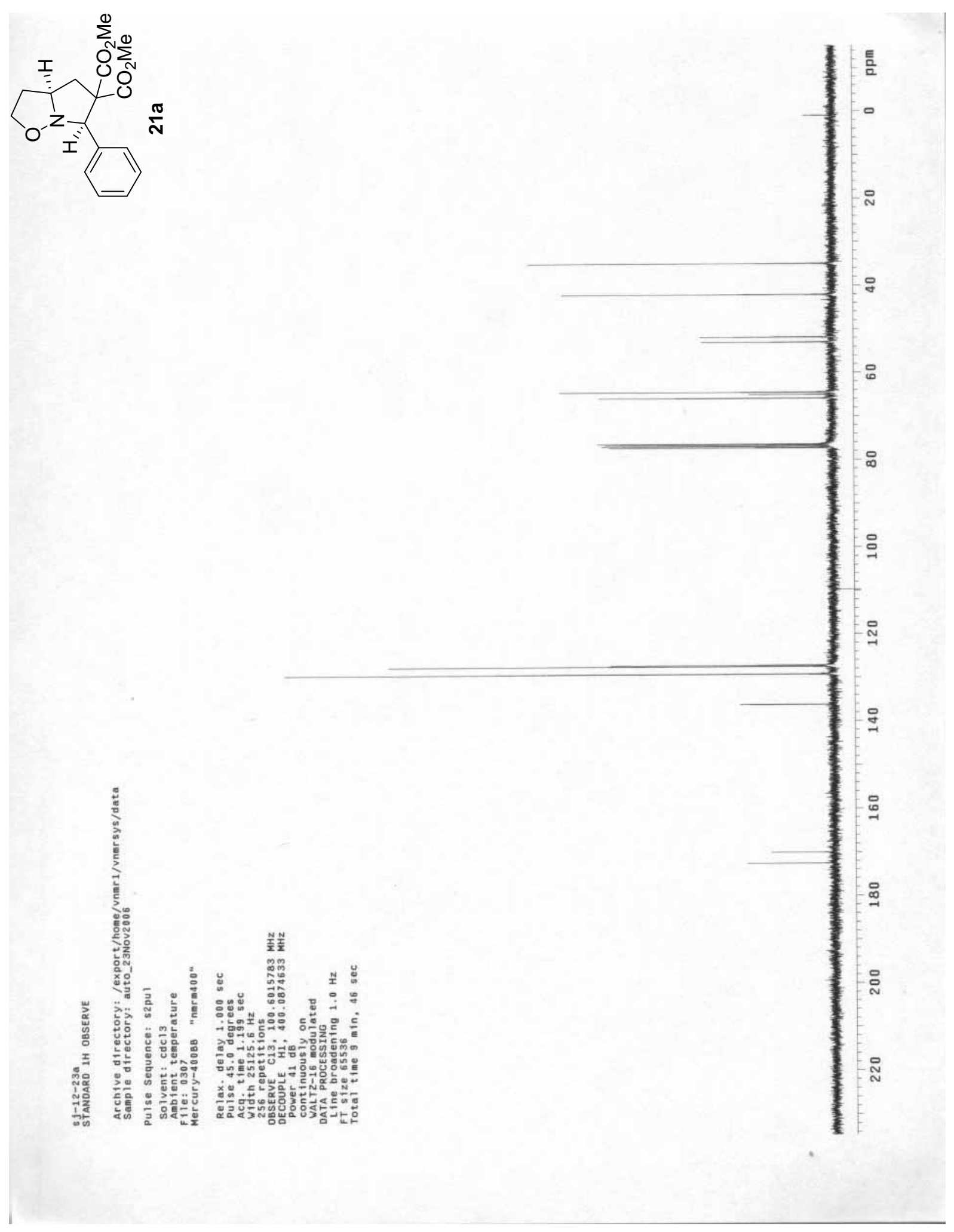




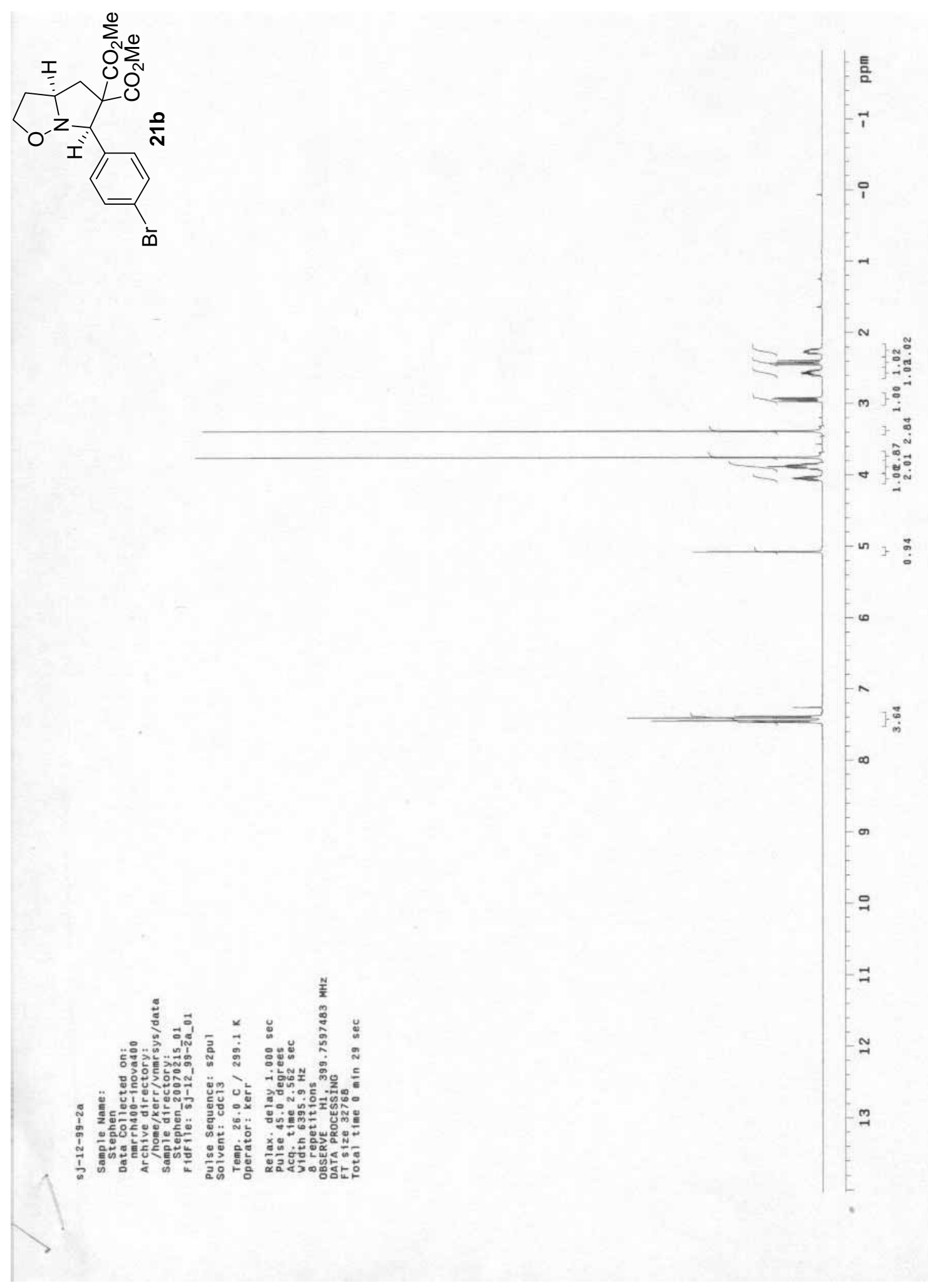



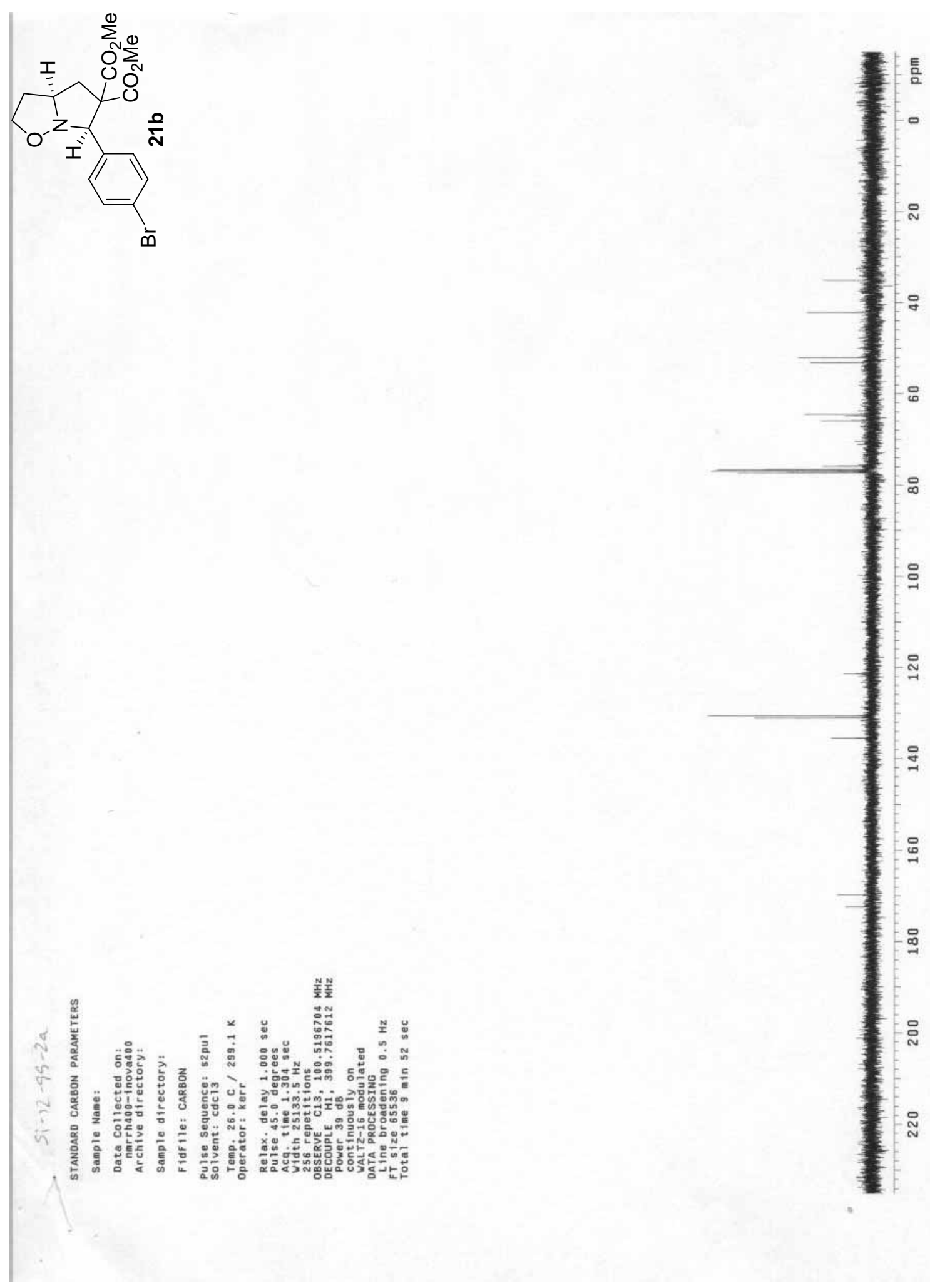

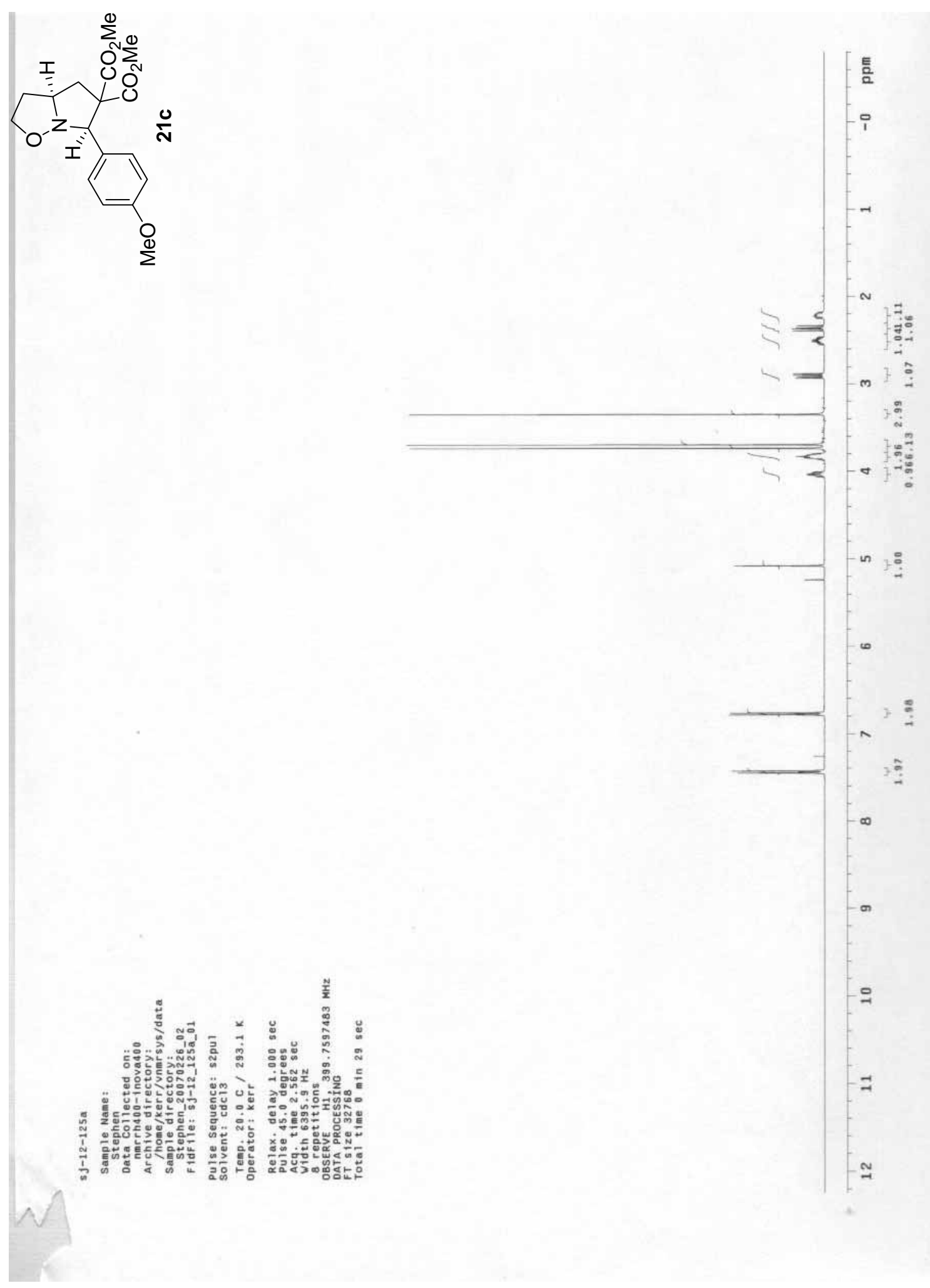


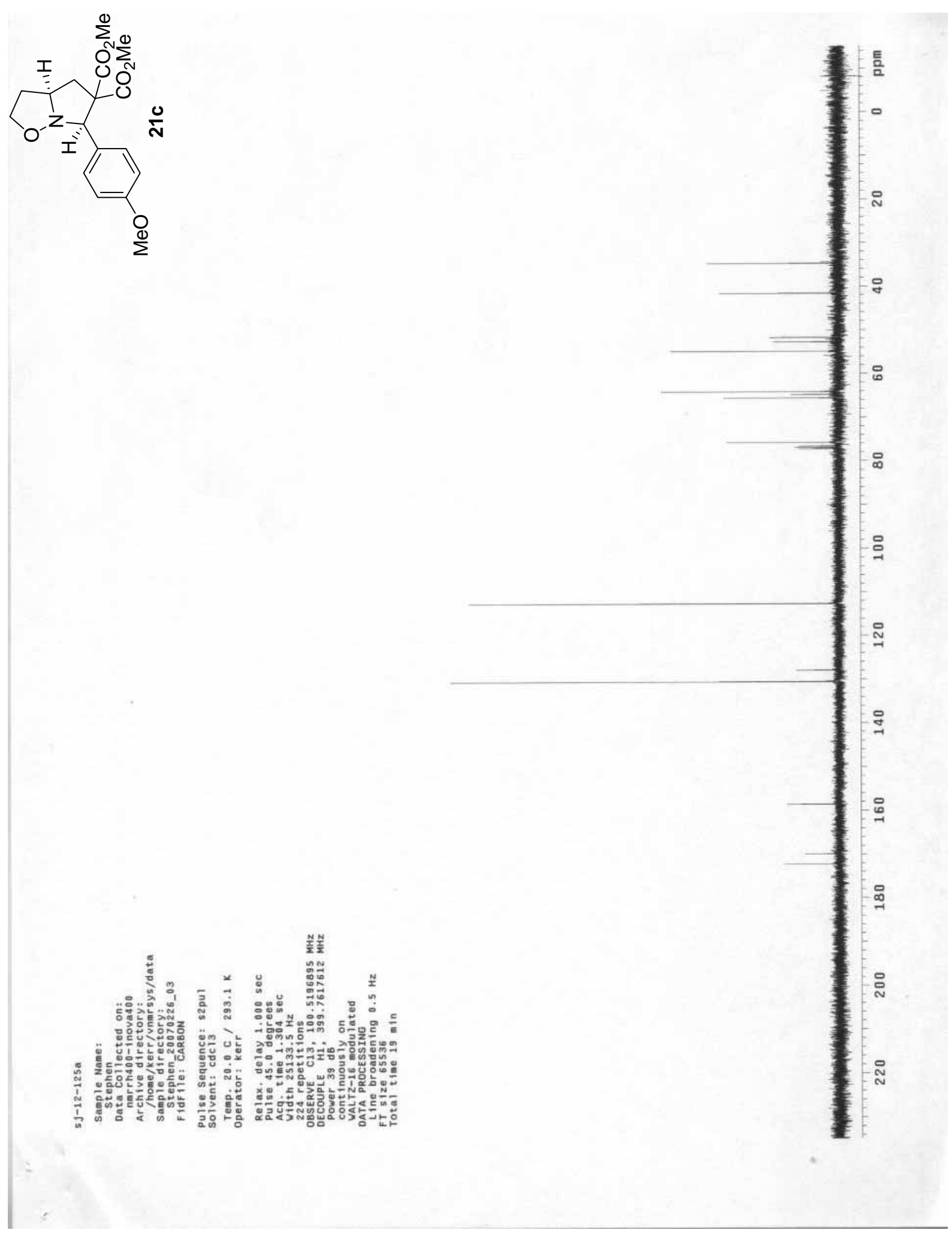




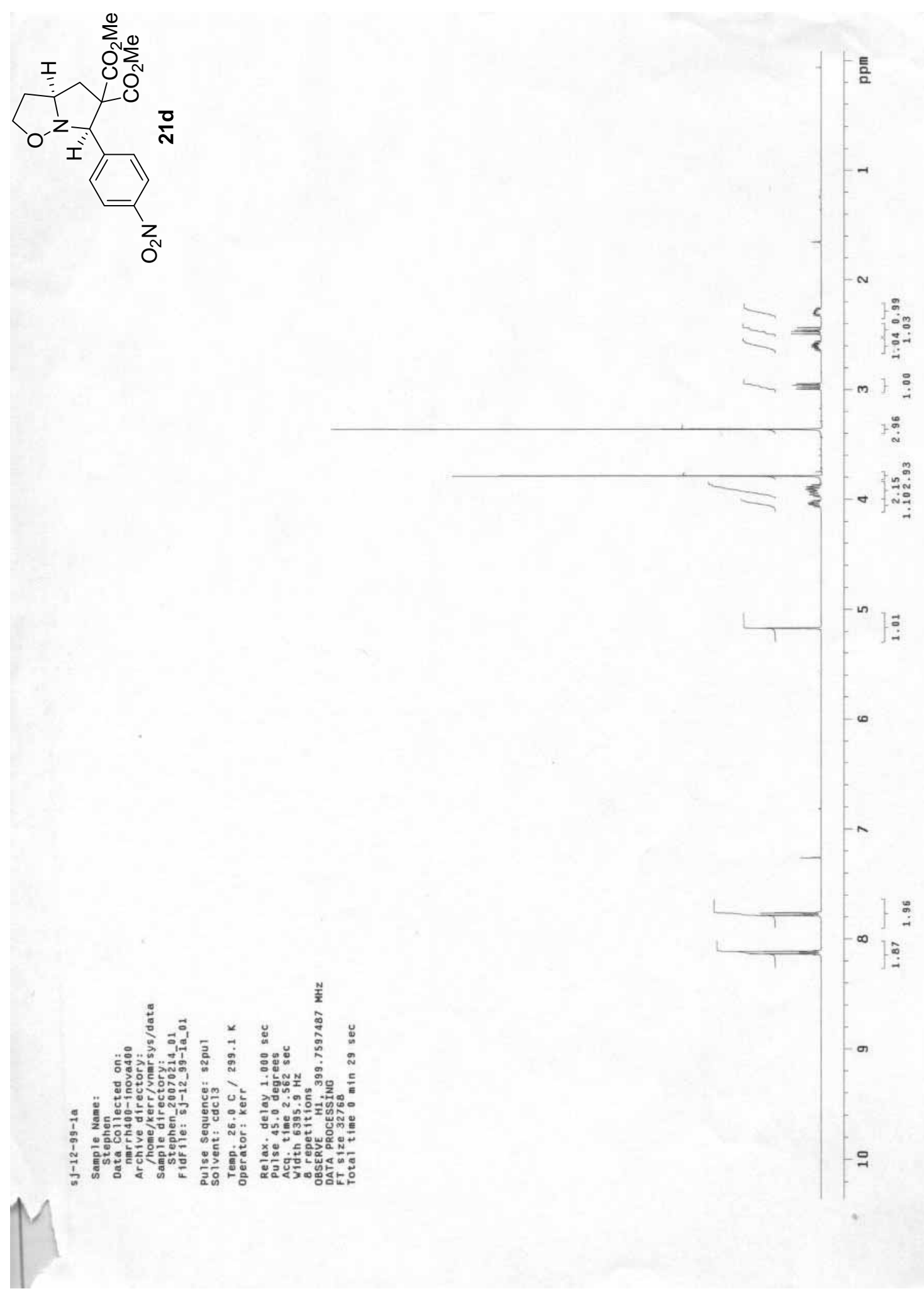



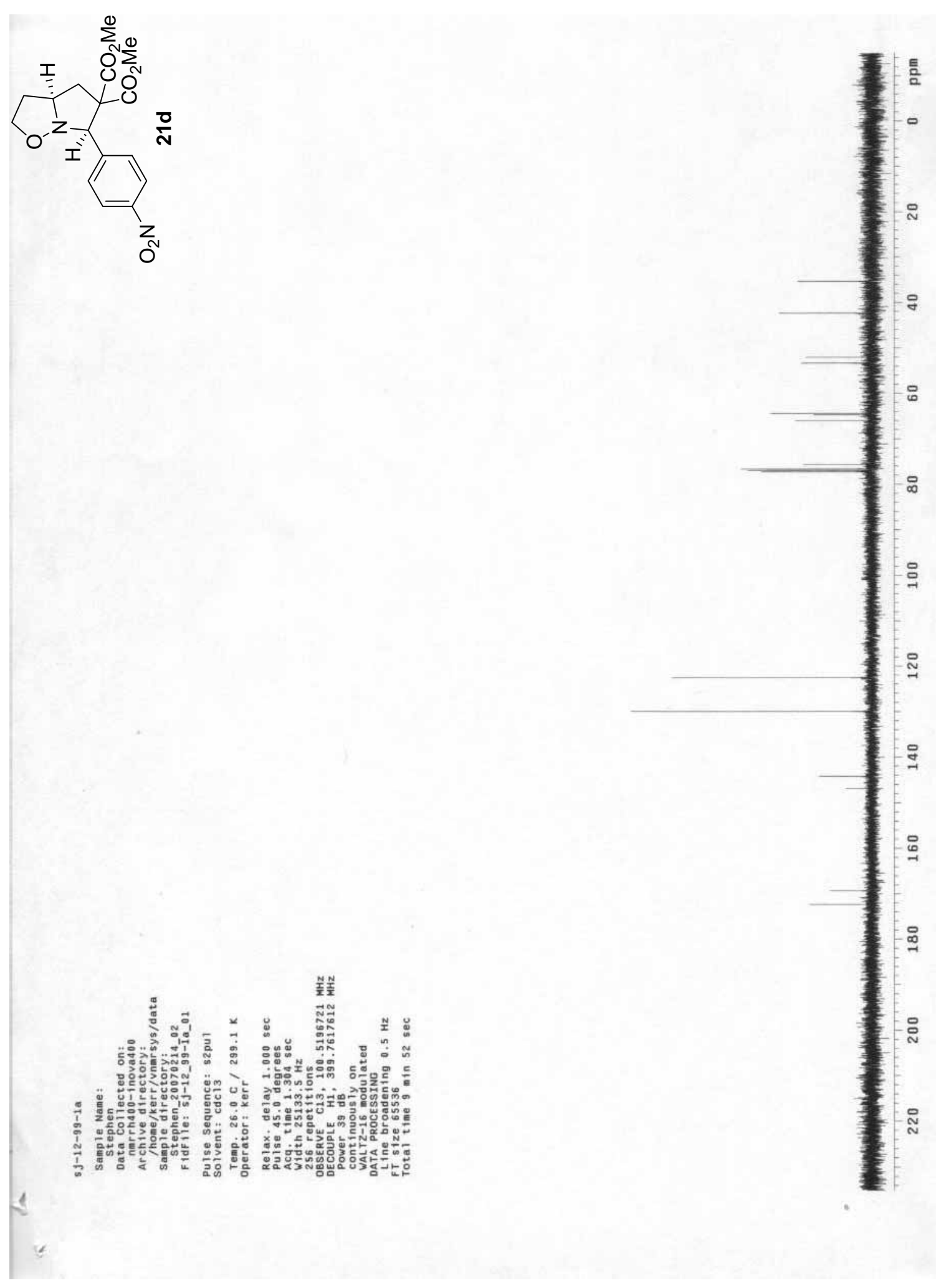


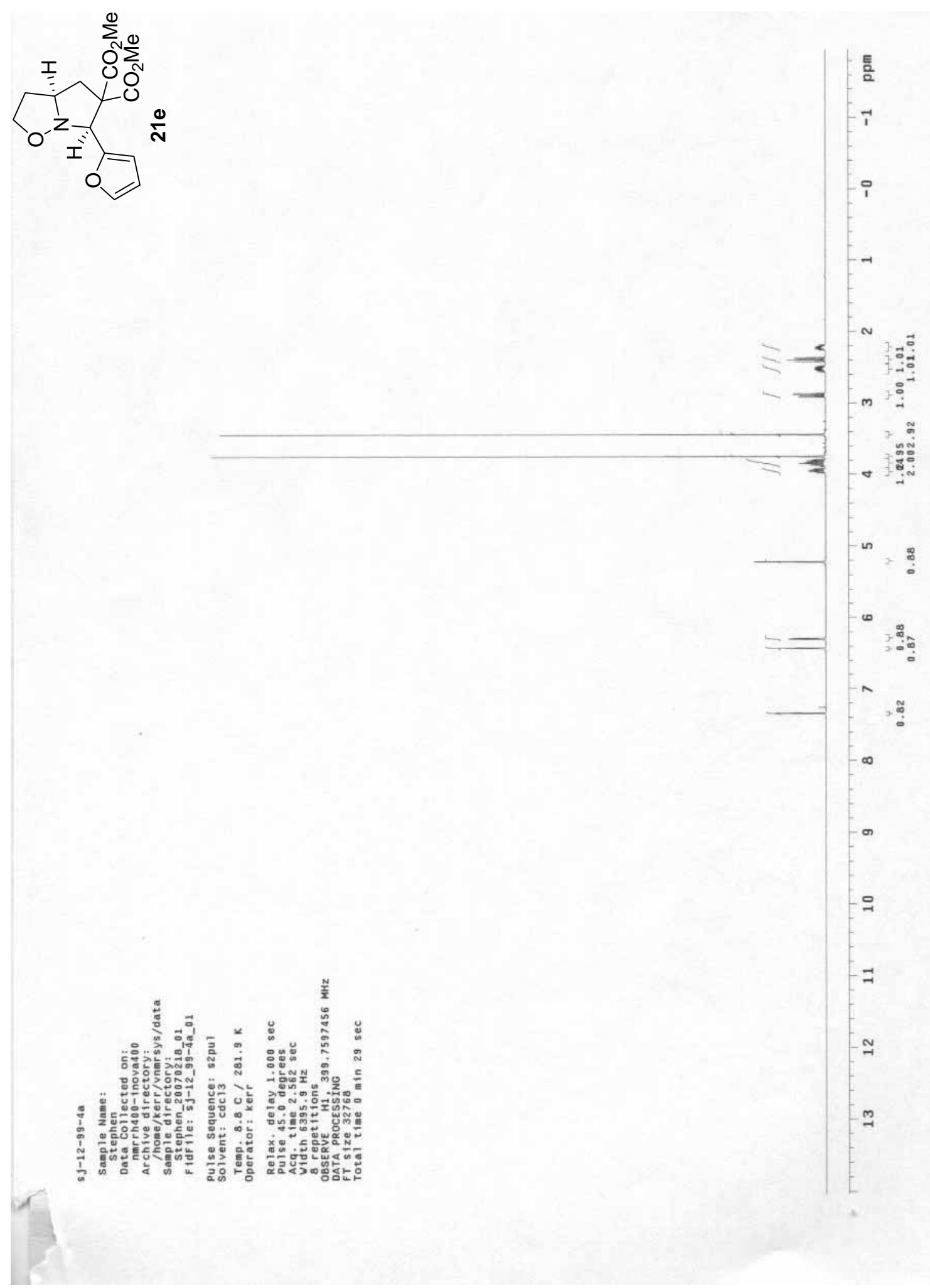



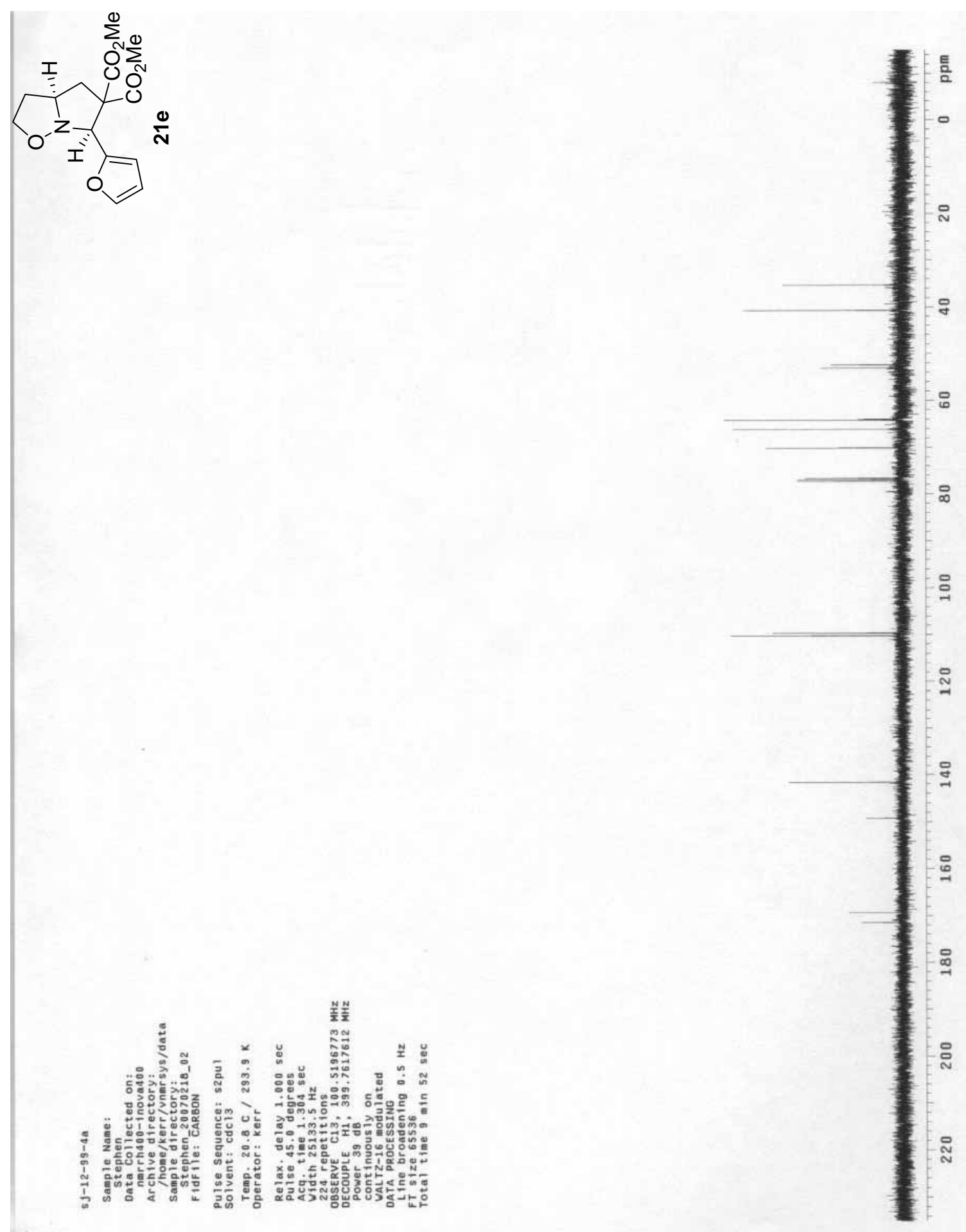


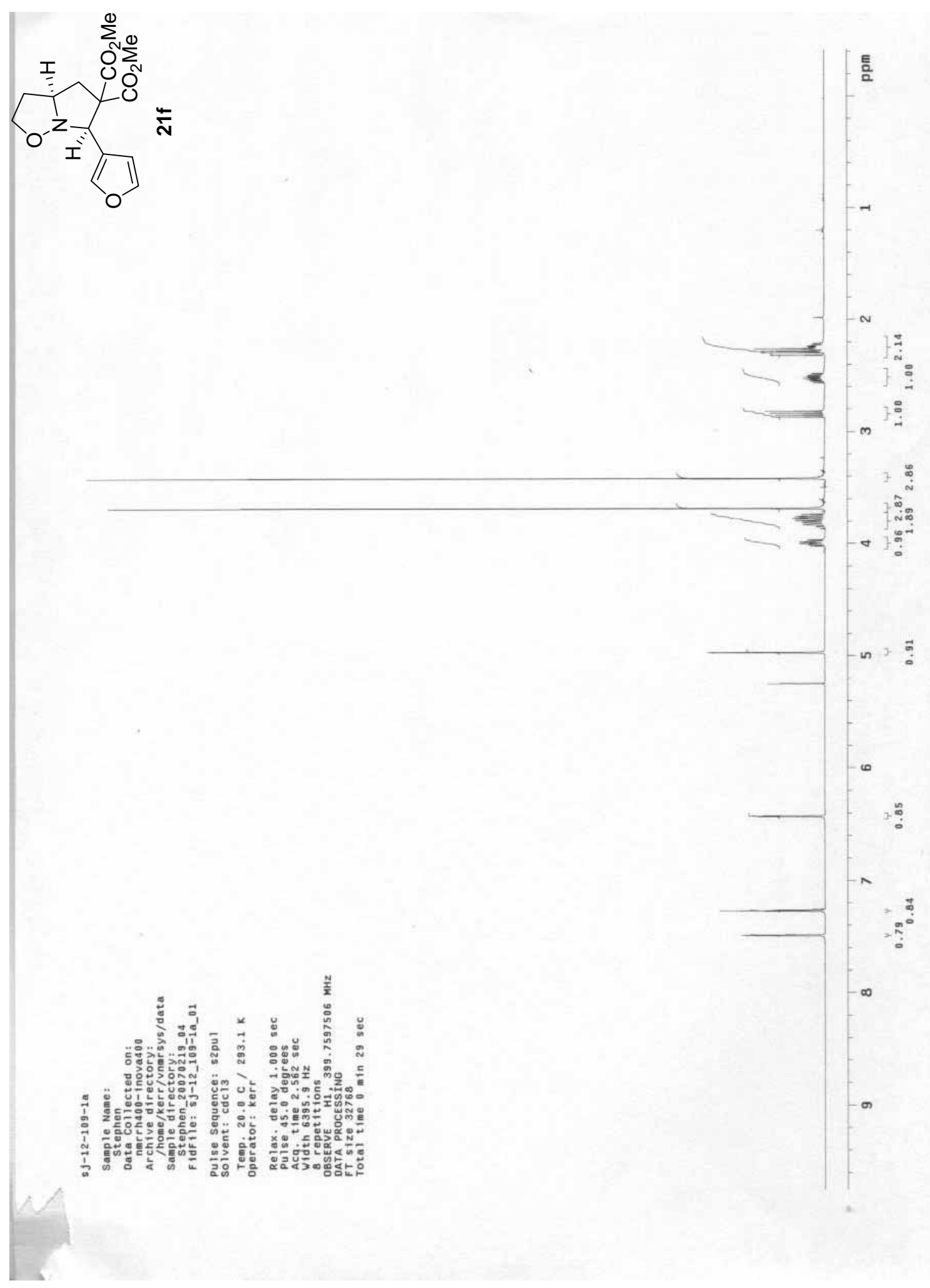




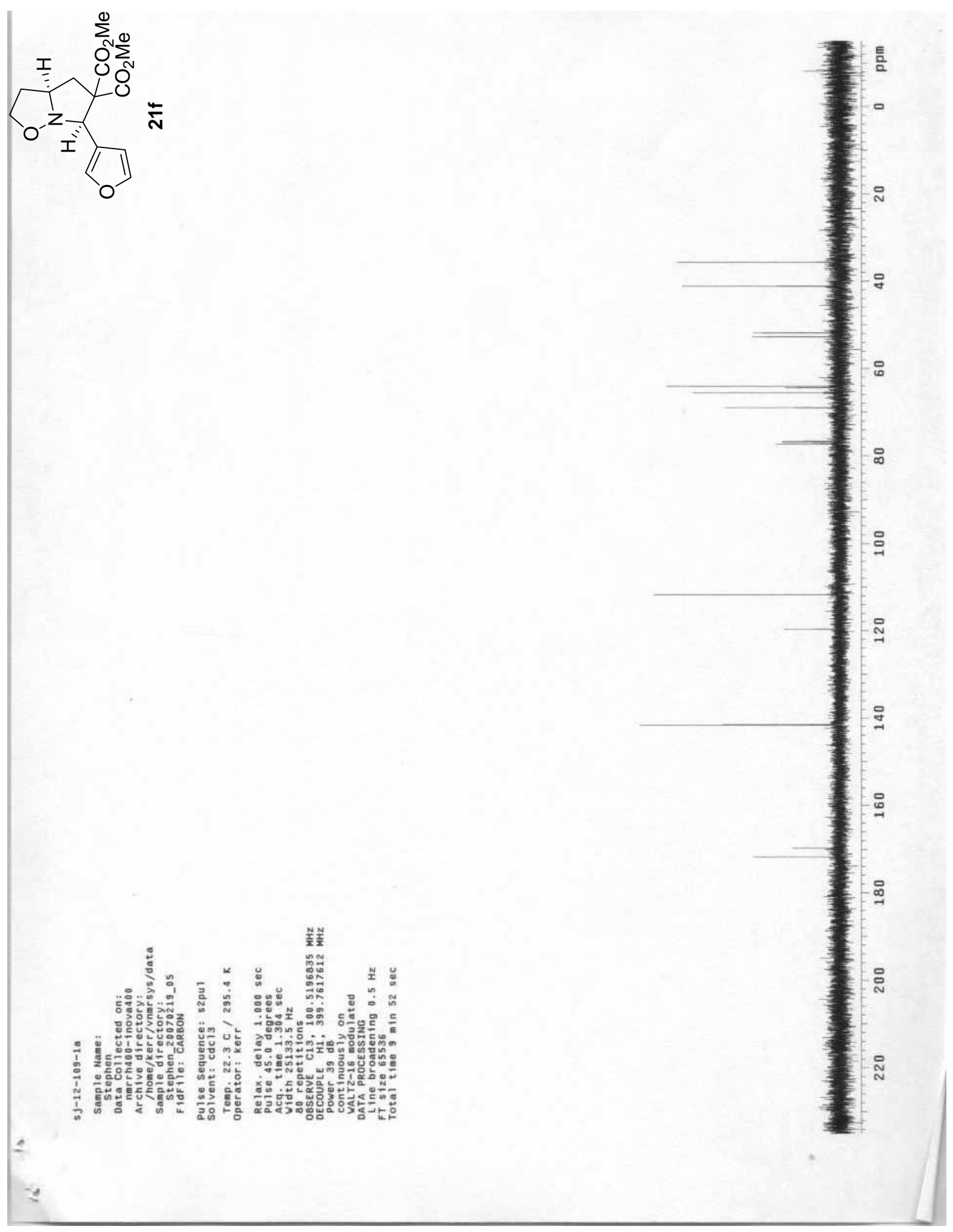



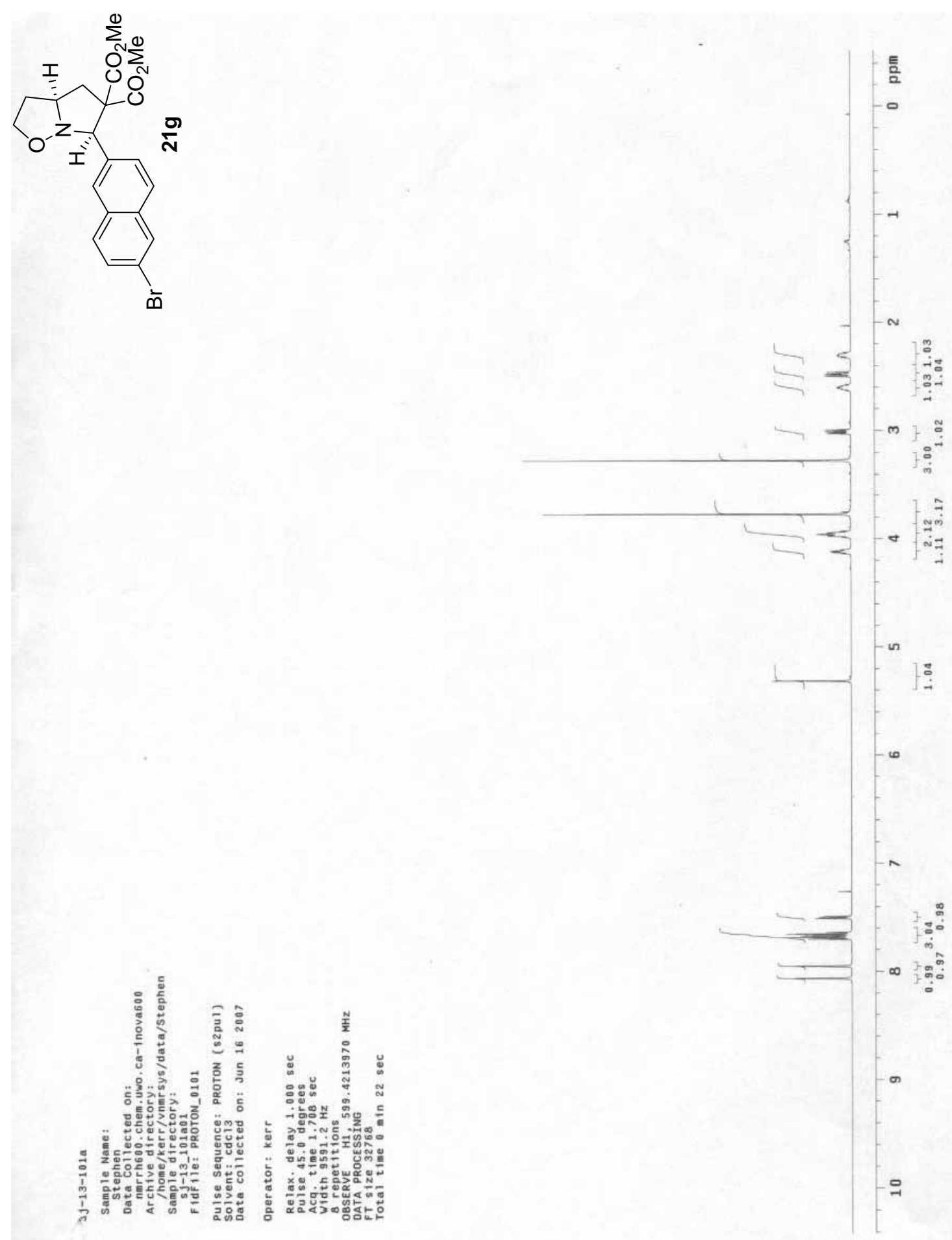

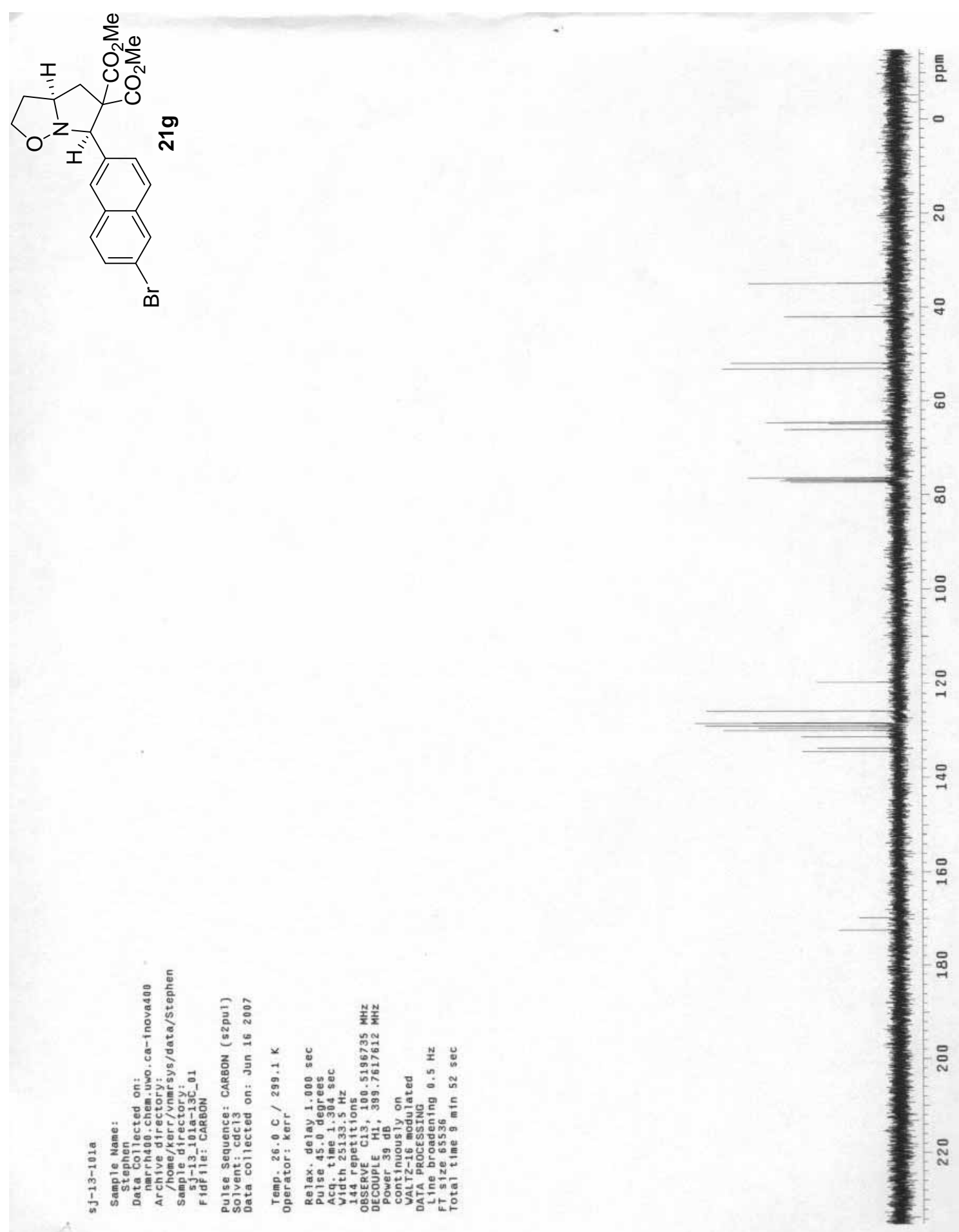


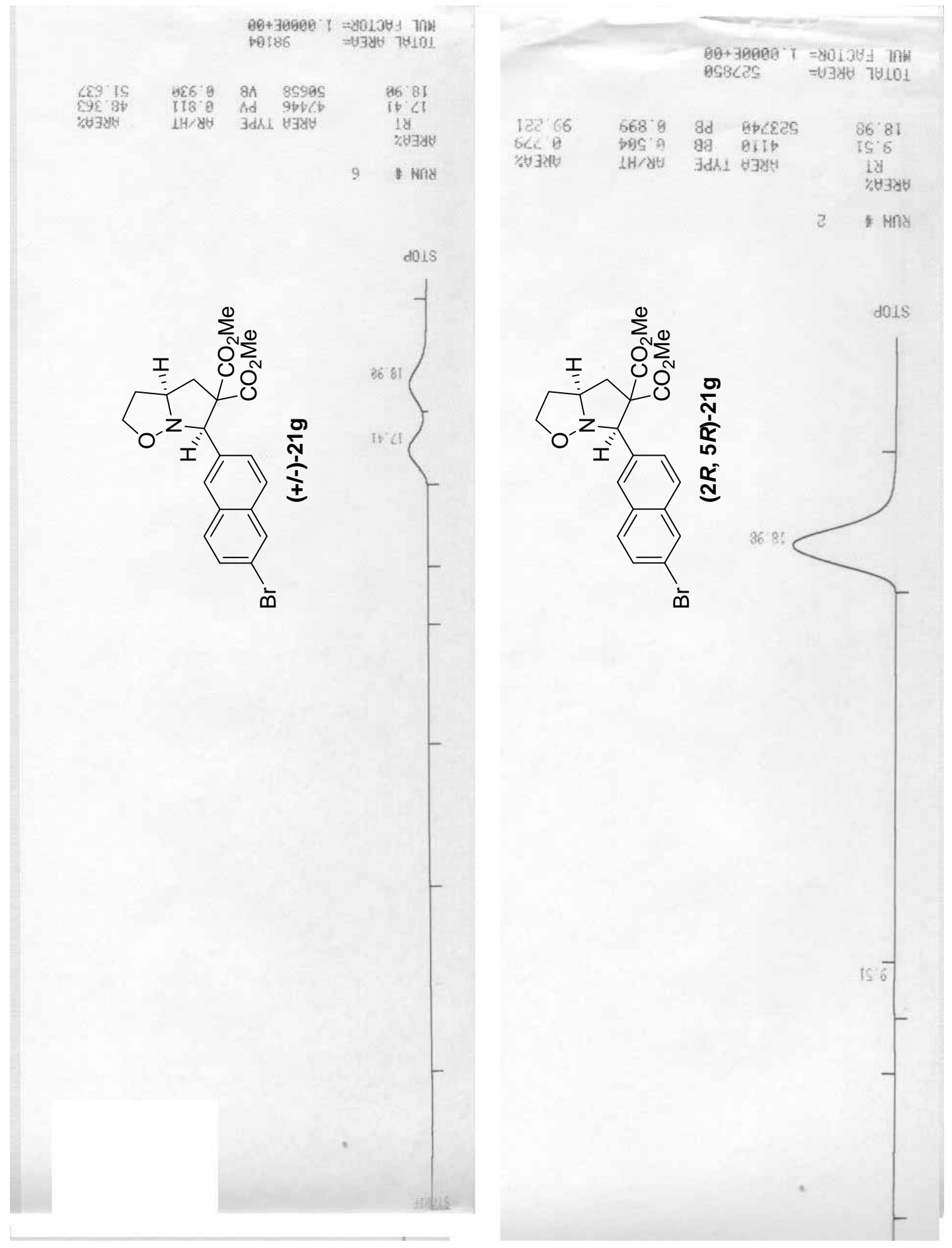



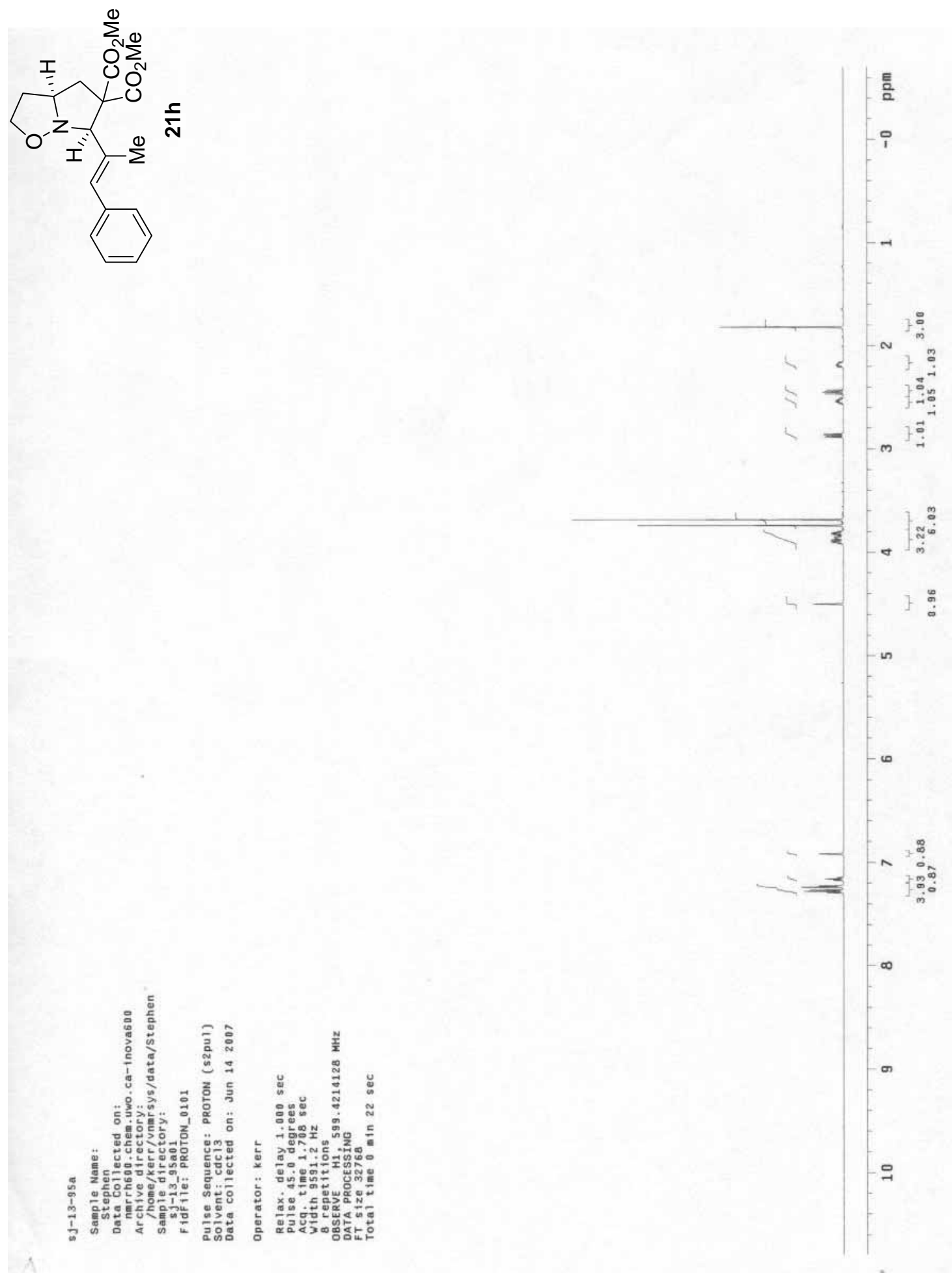

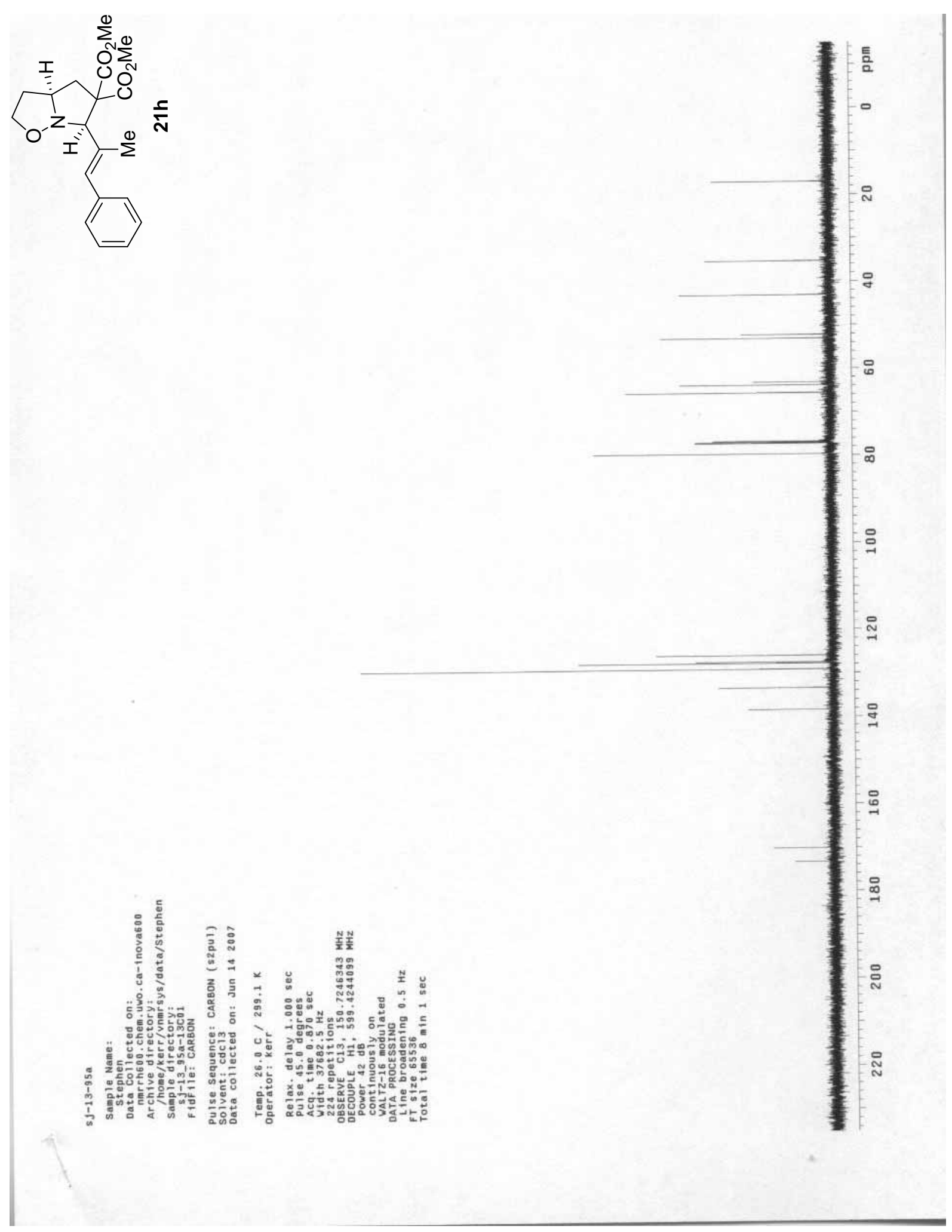

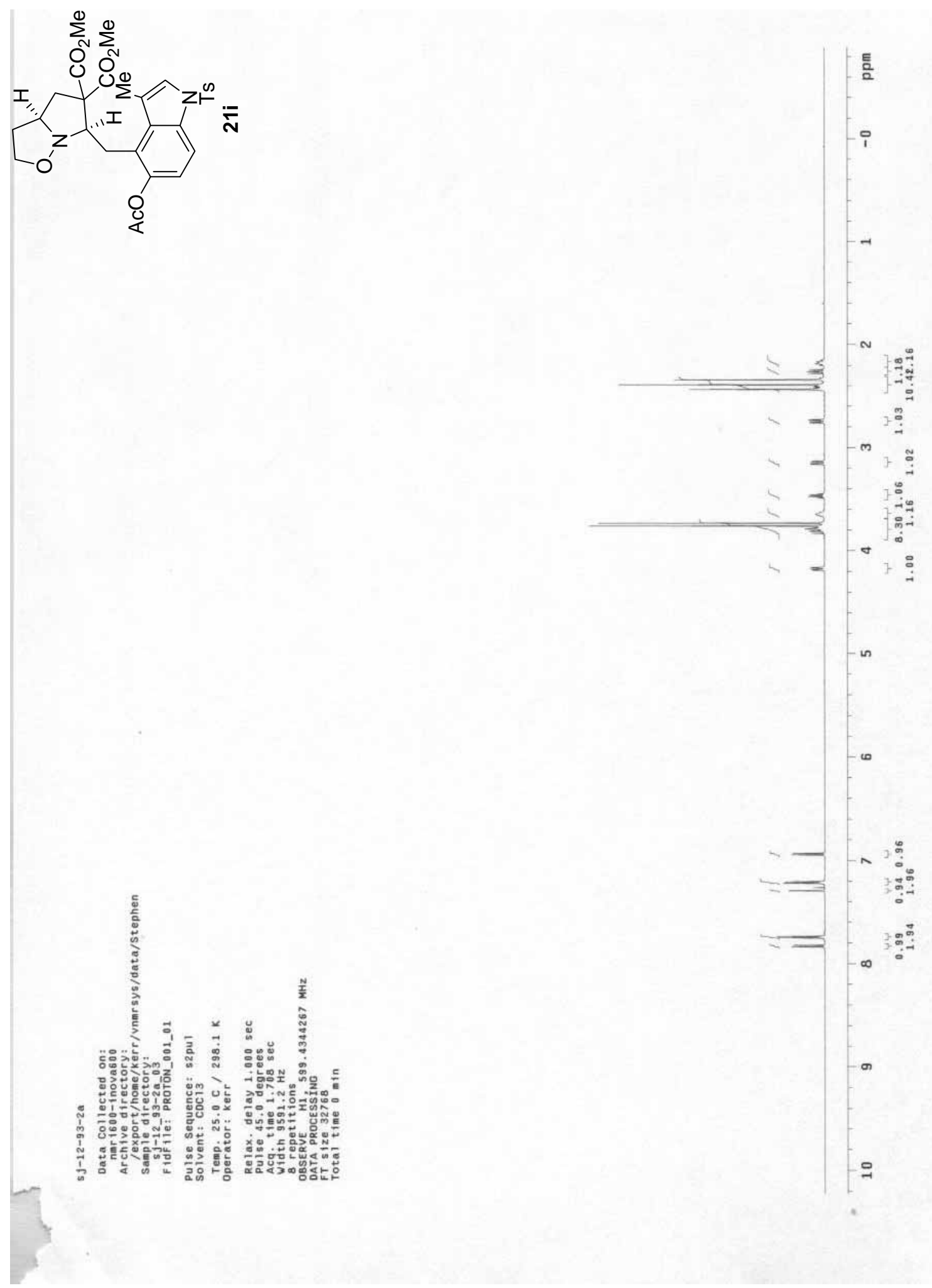


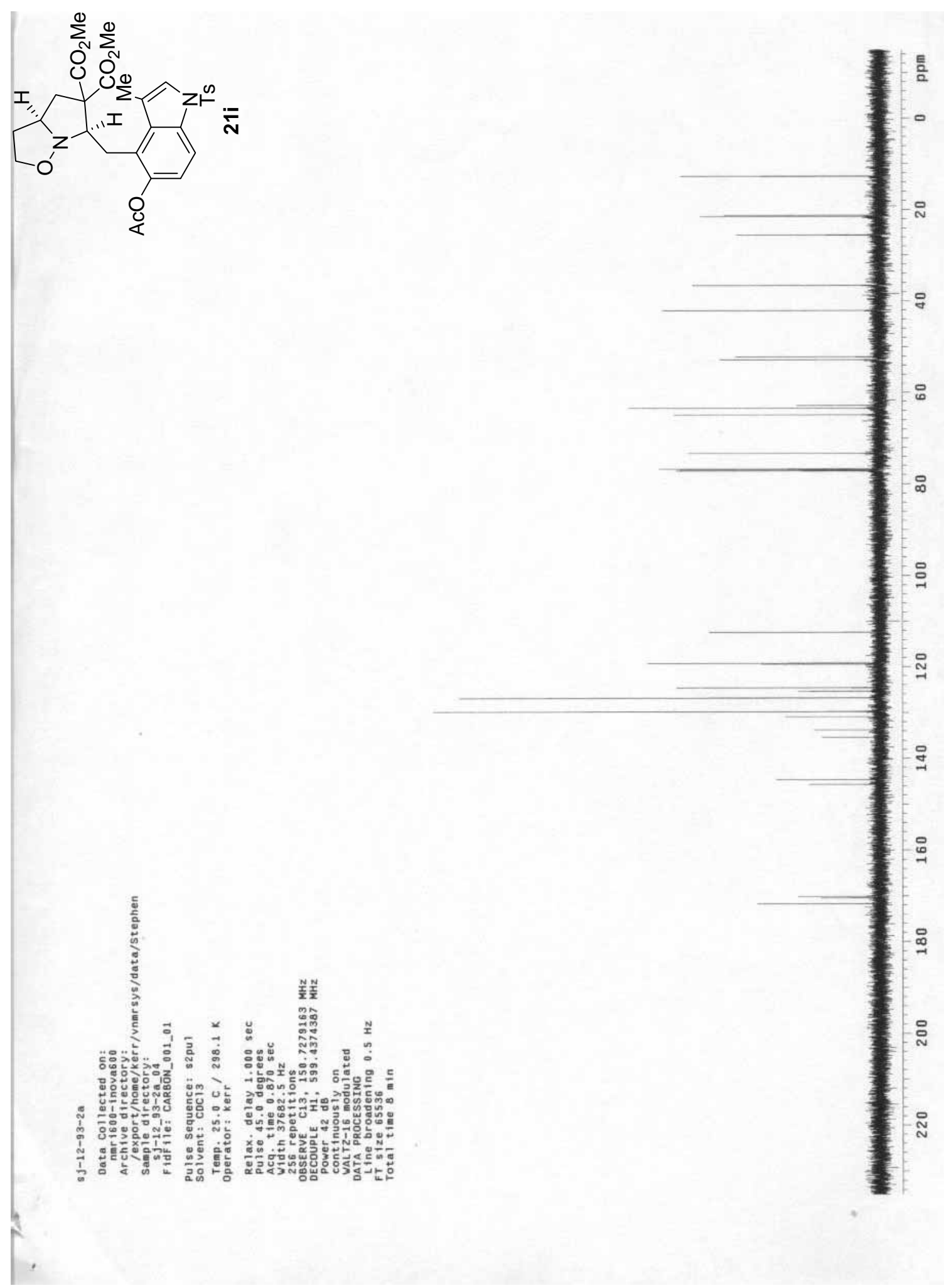



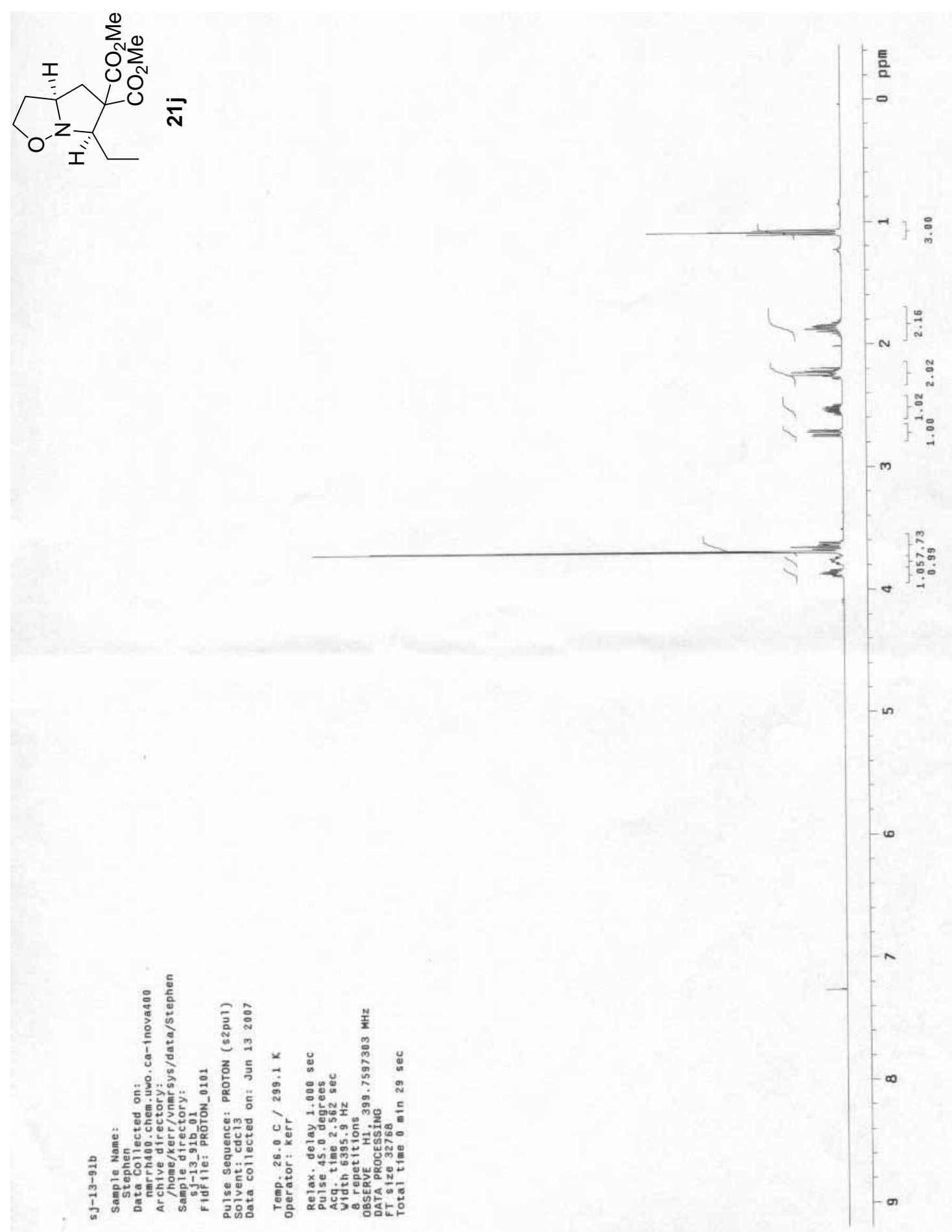

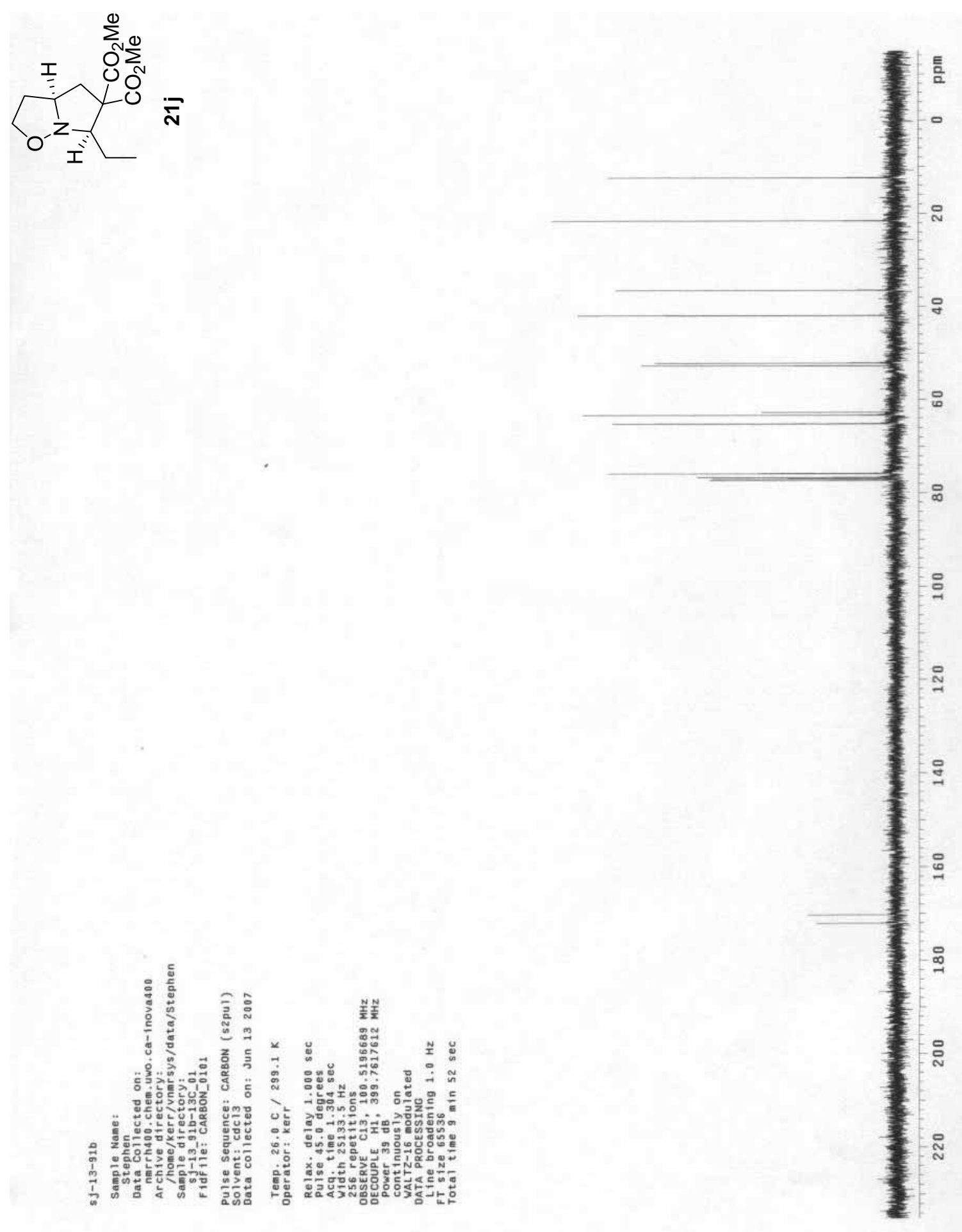


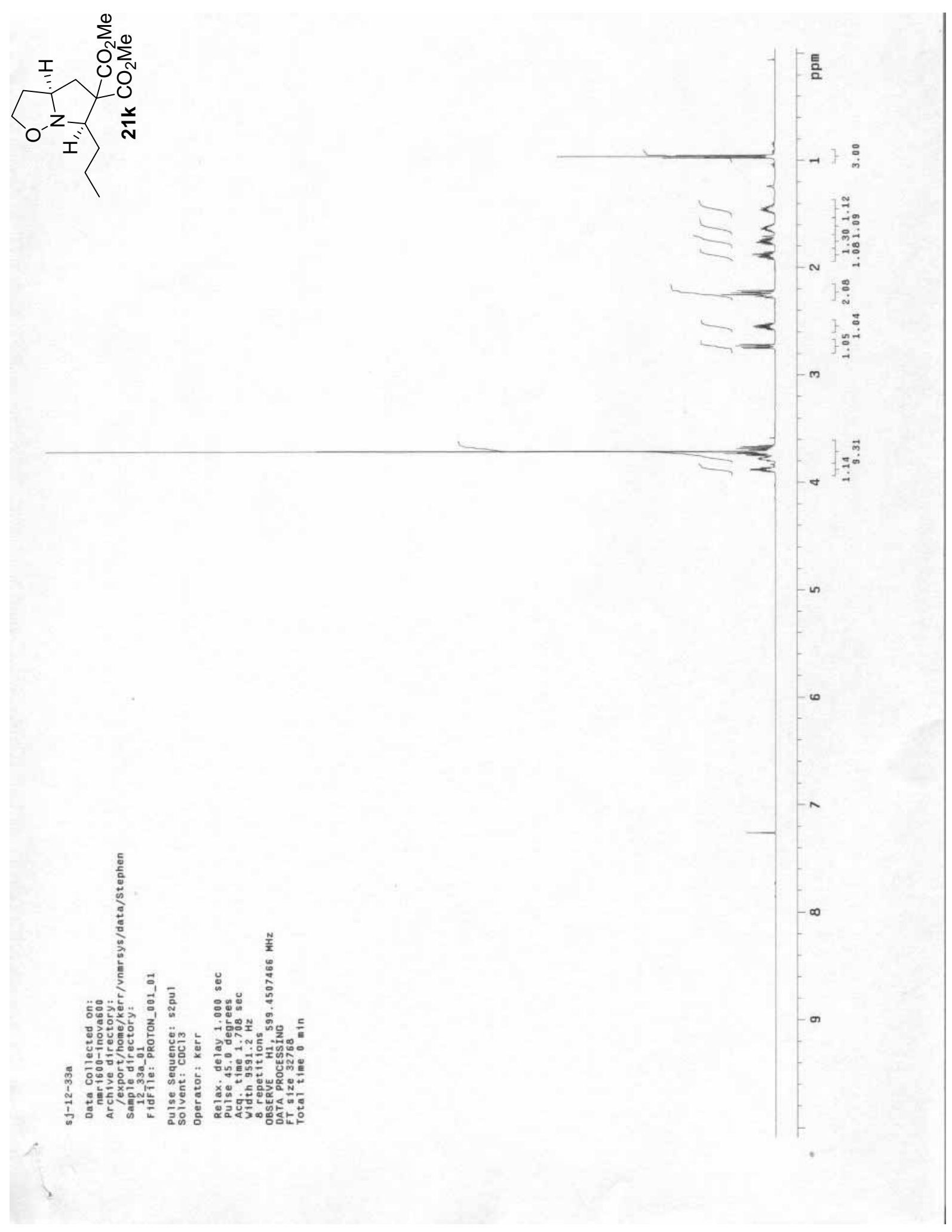



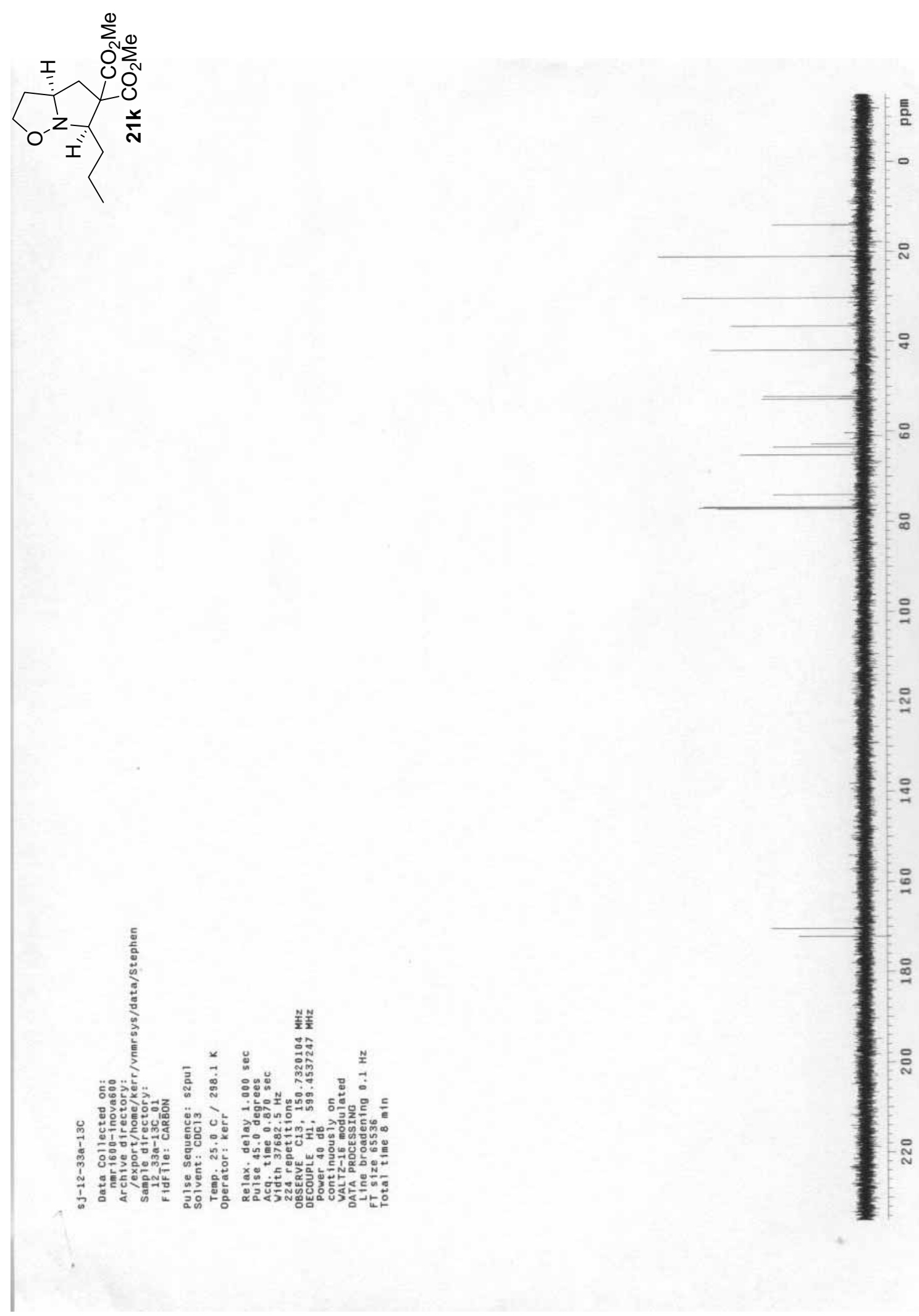

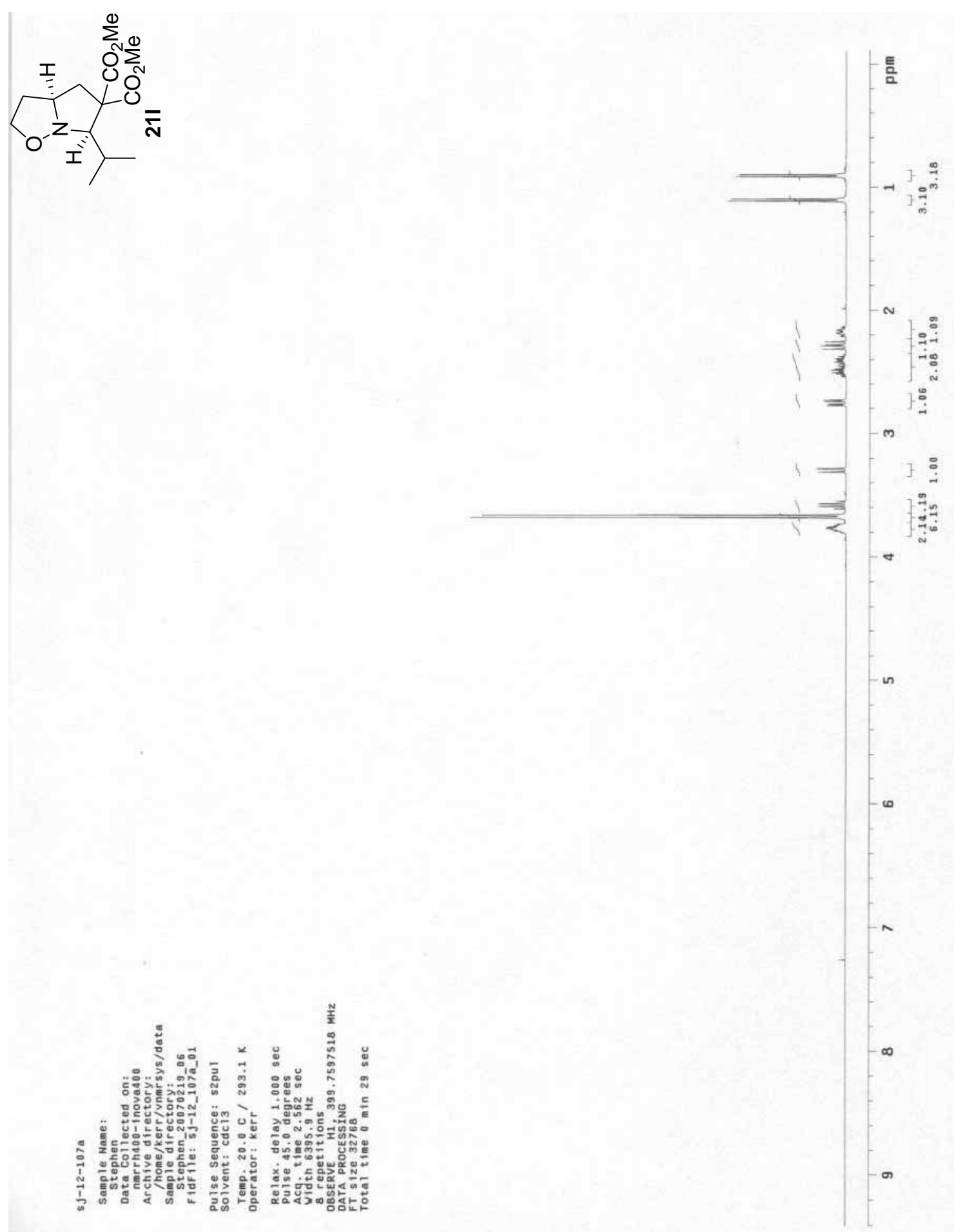

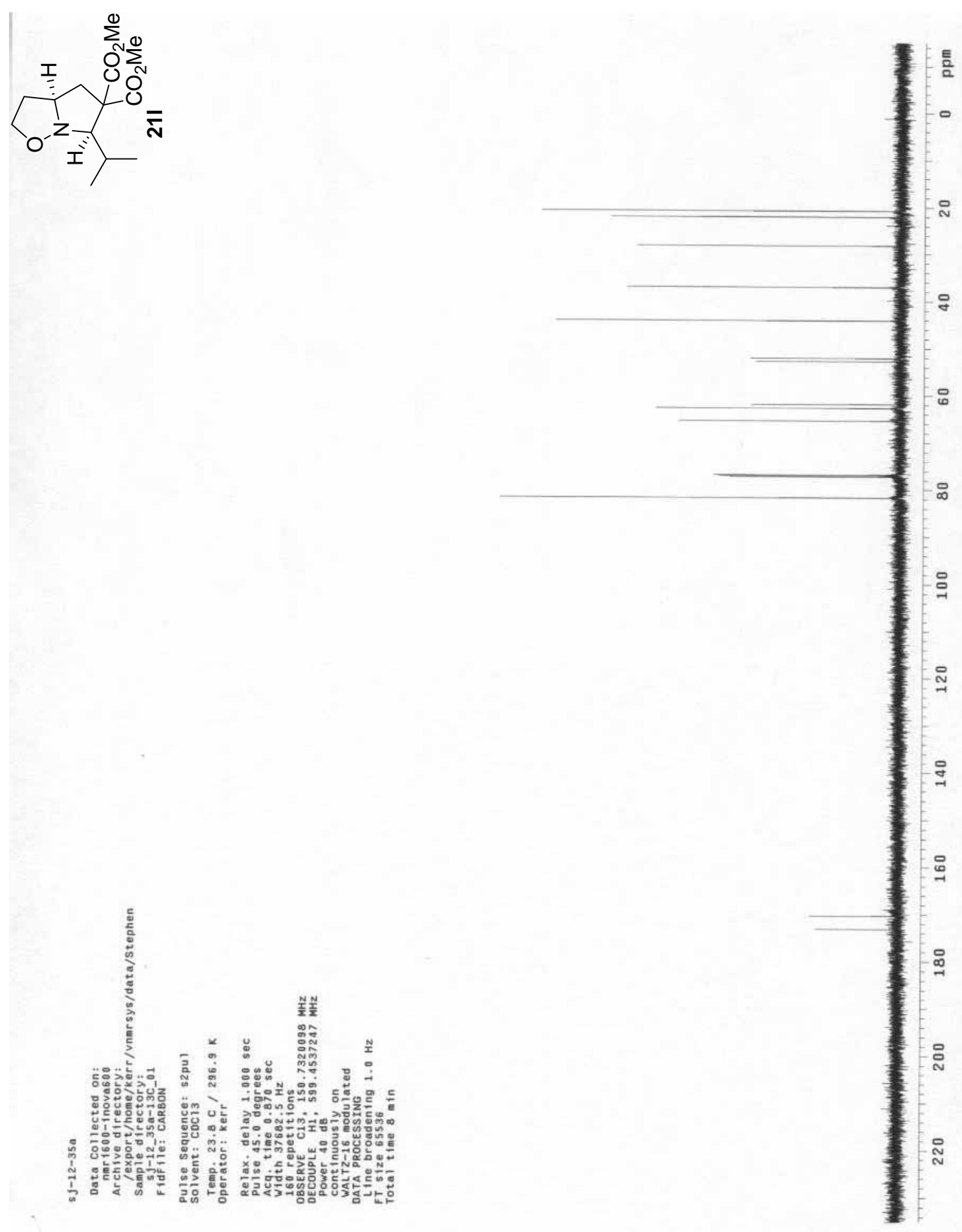


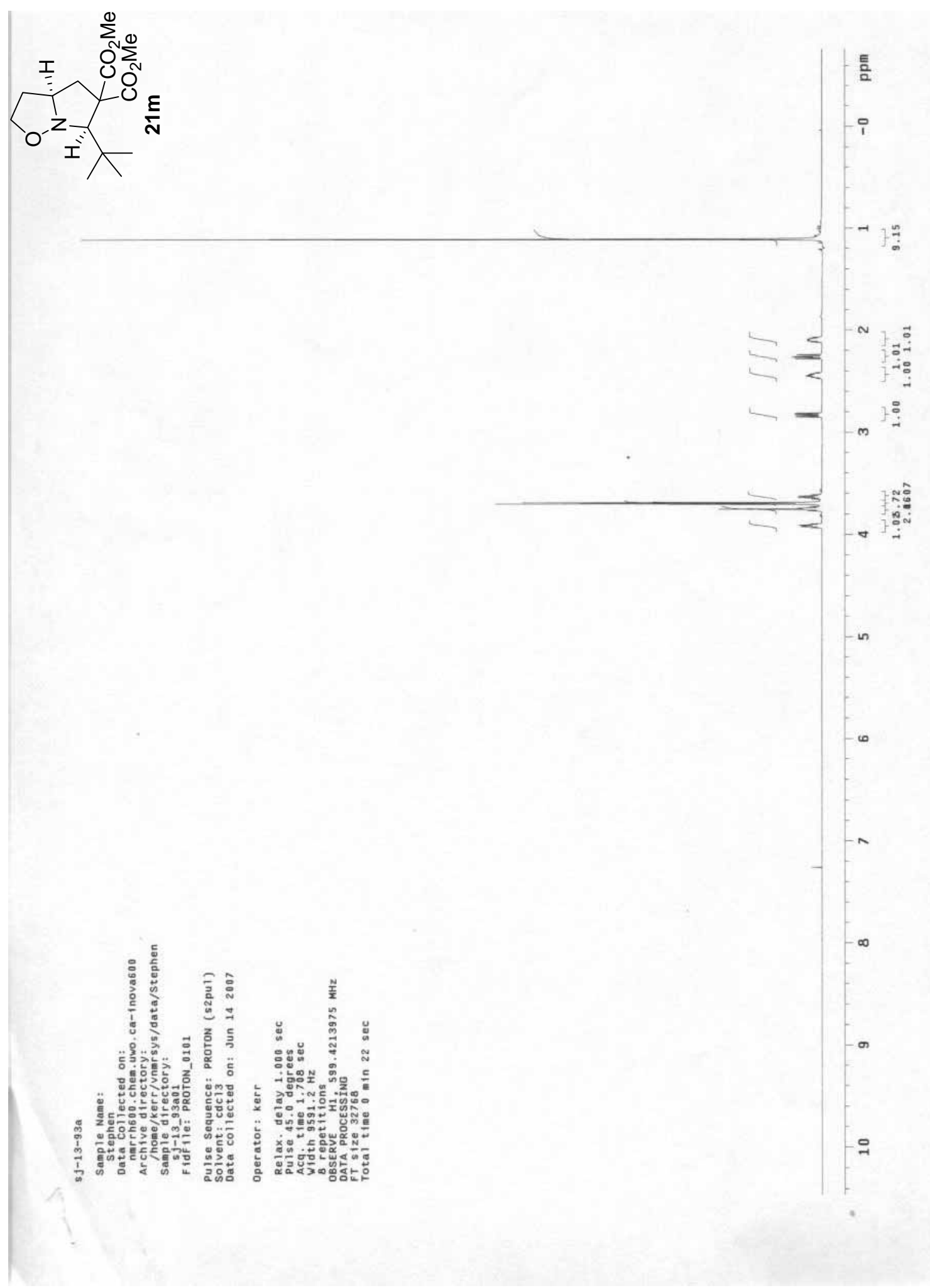




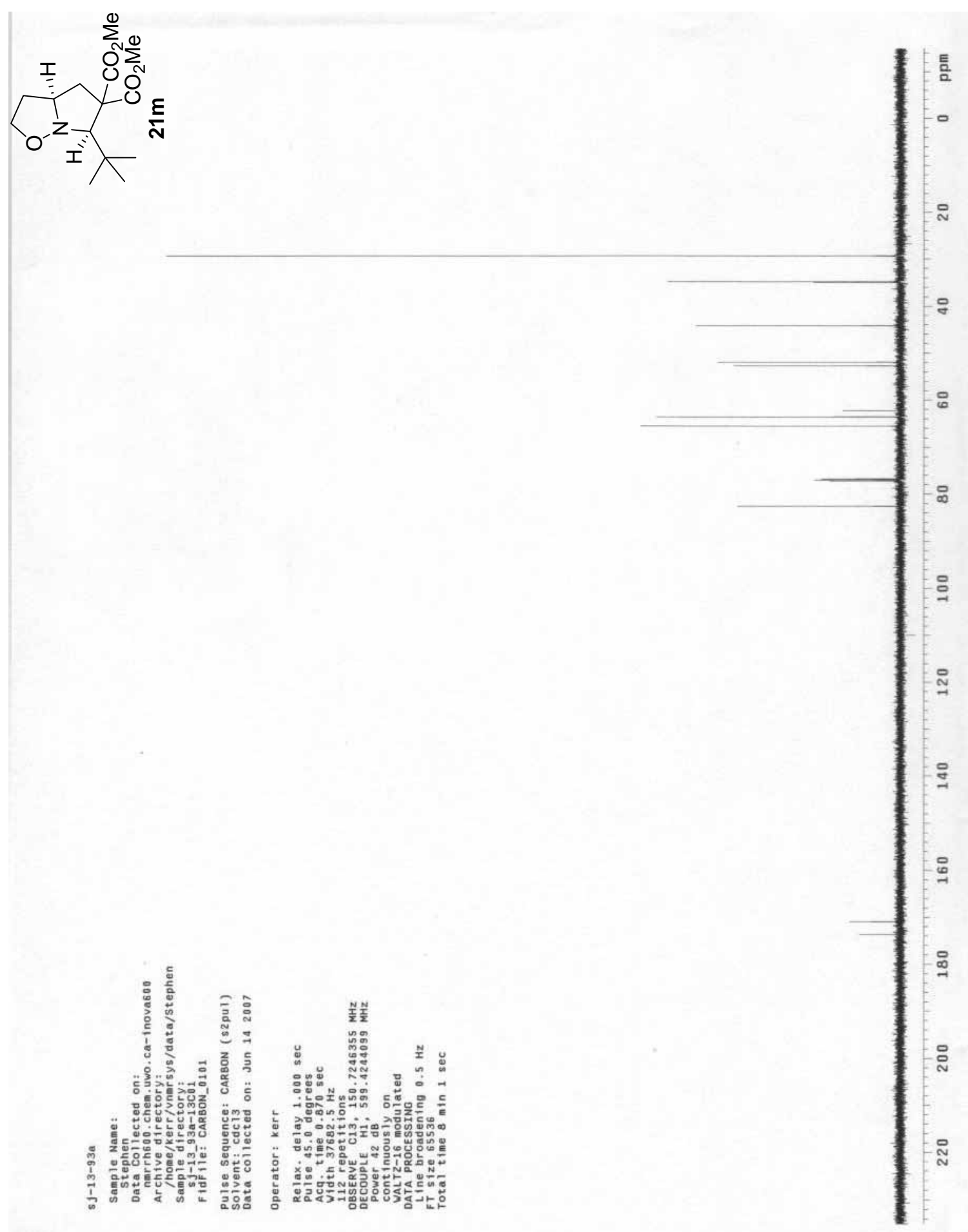



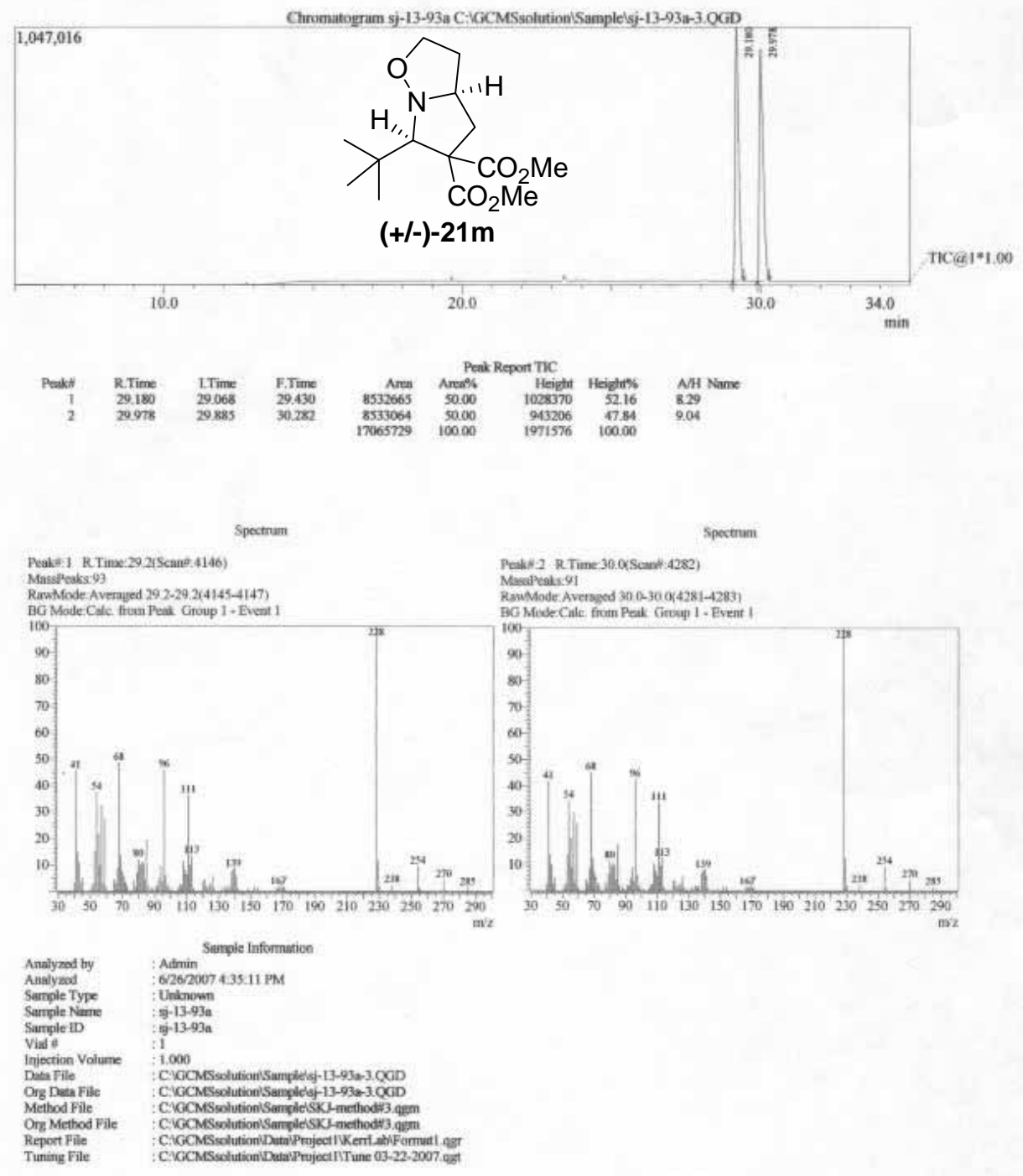


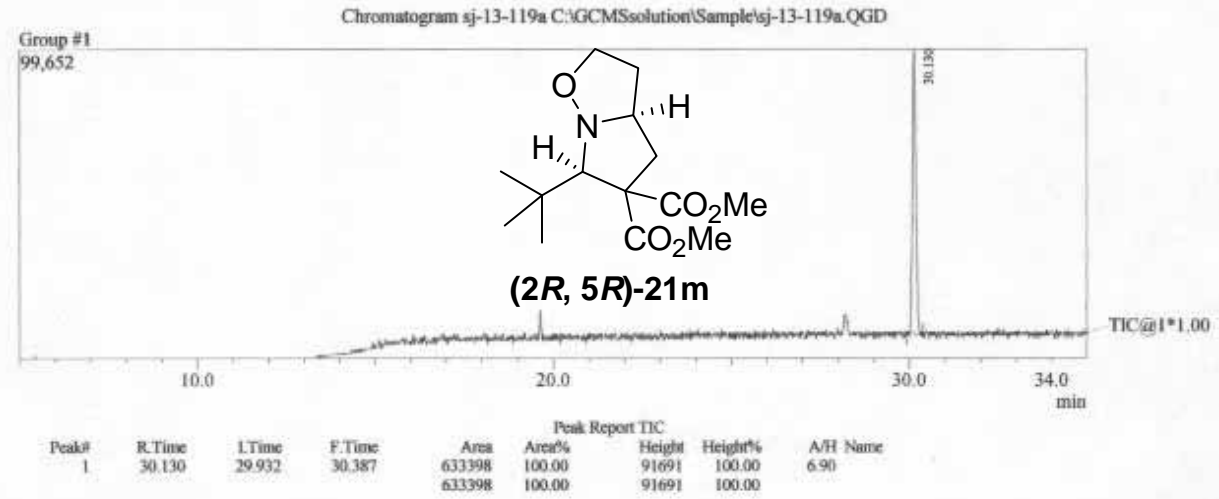

Peake, I RTime: 30 1(Scant:-4306)
Masspeaks 35

Rawhlode Averayed 30 1-31:1(4307-1300)

ba Mode Canc fon
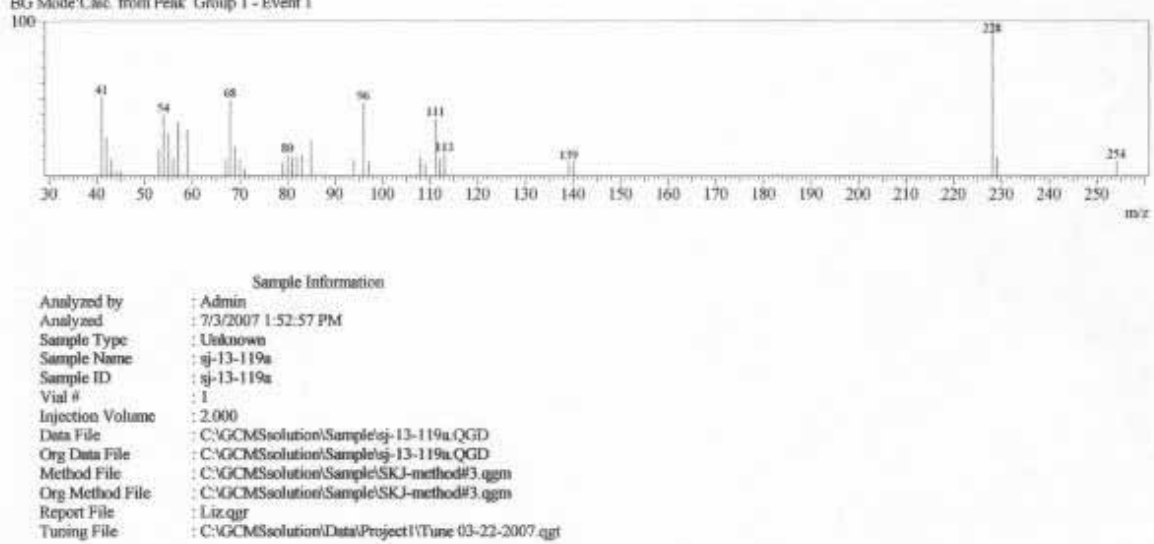

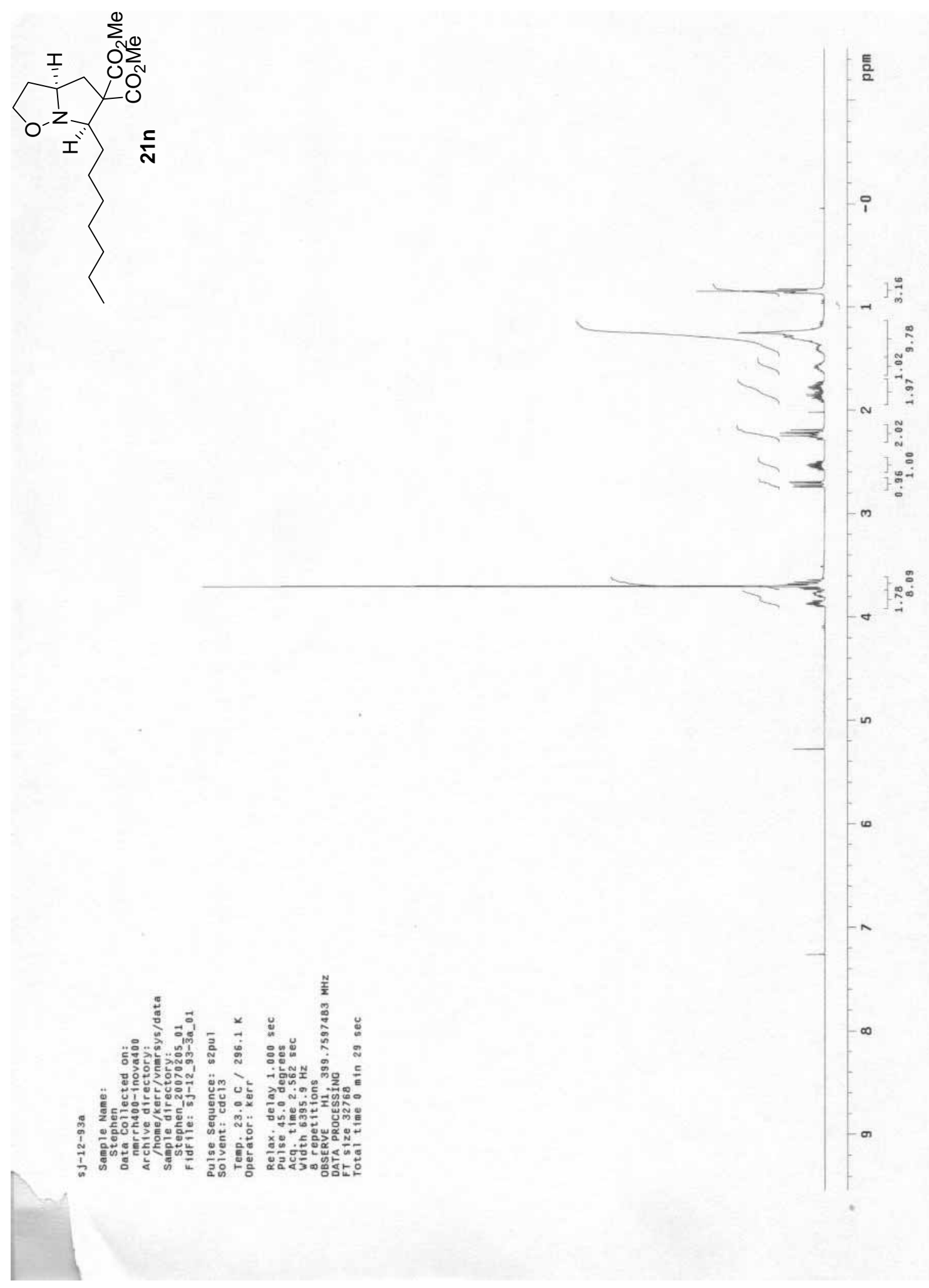


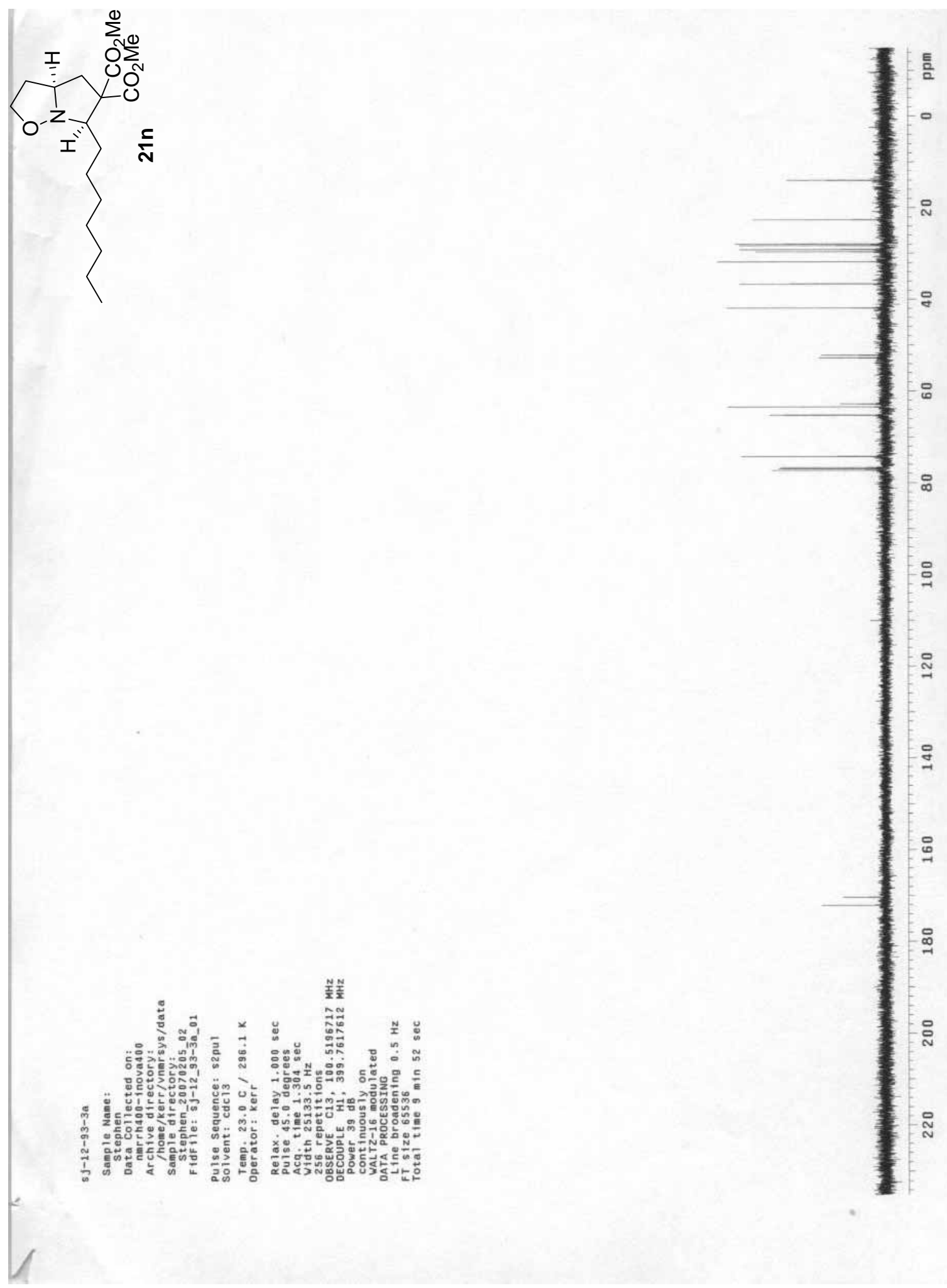




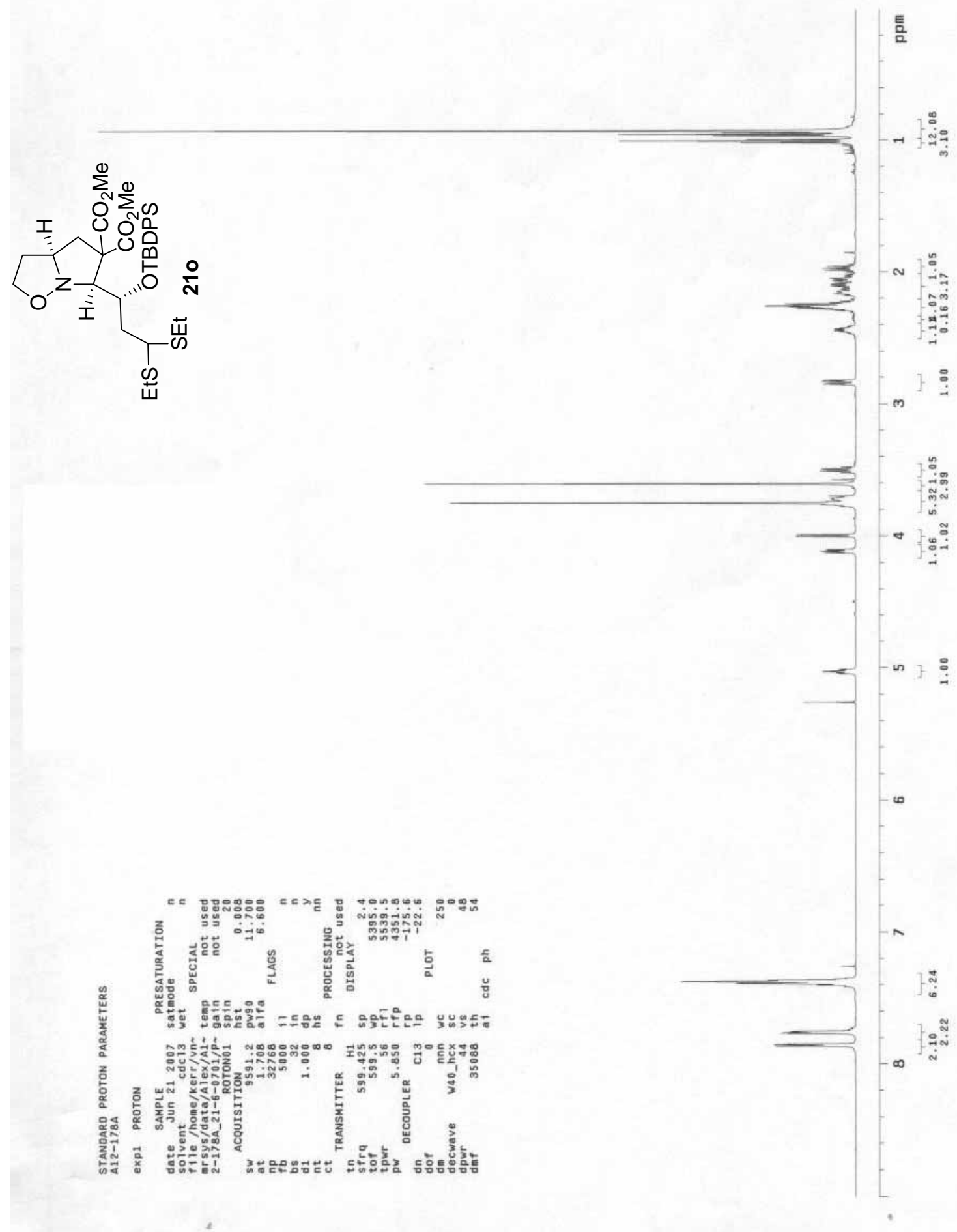



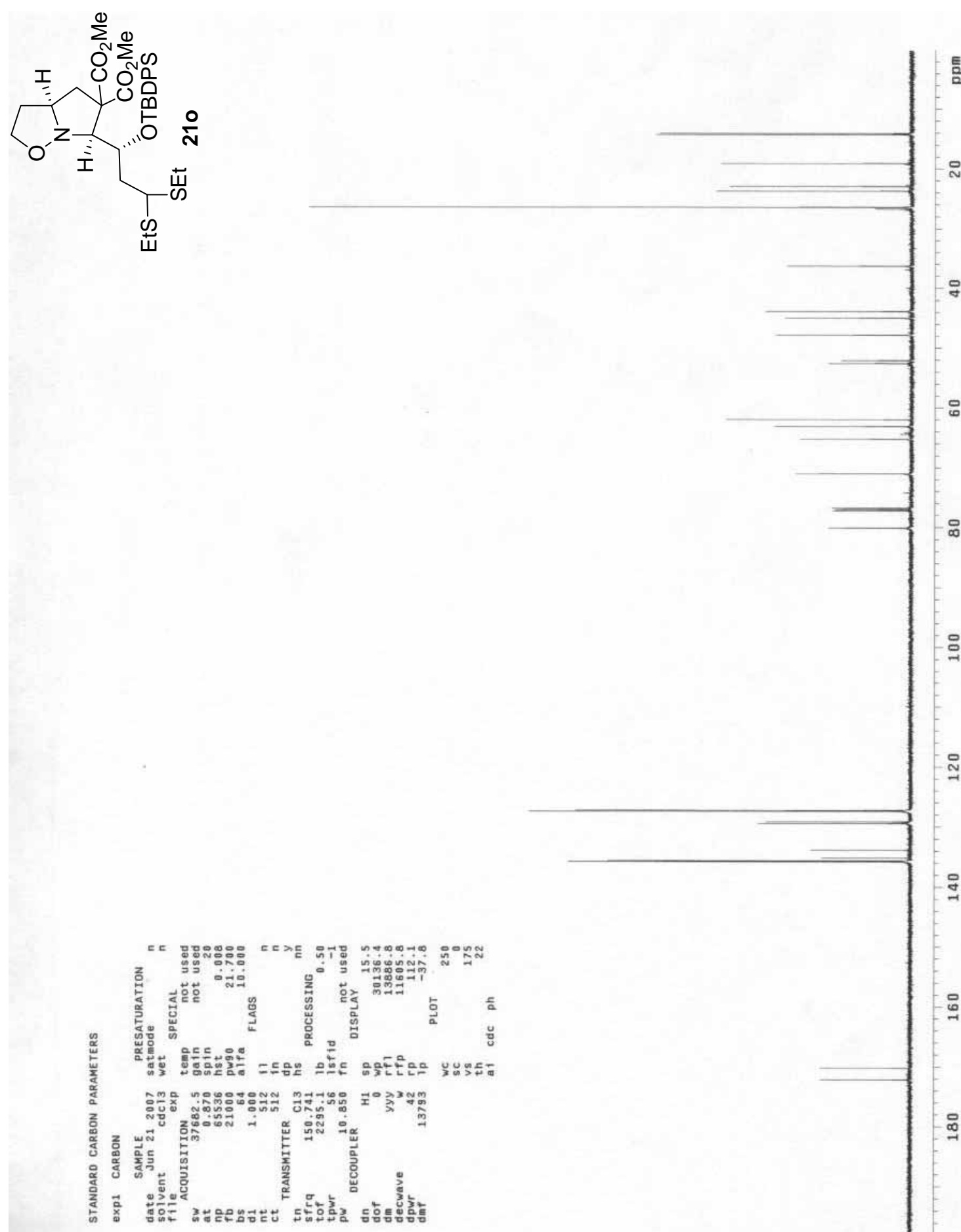

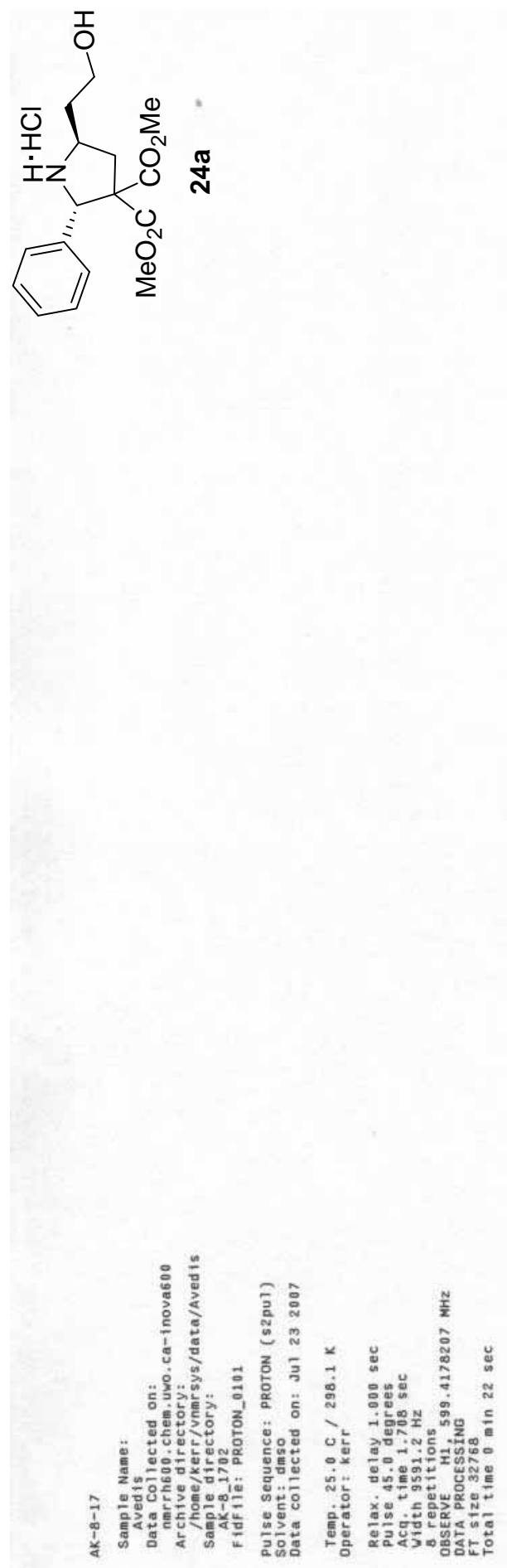

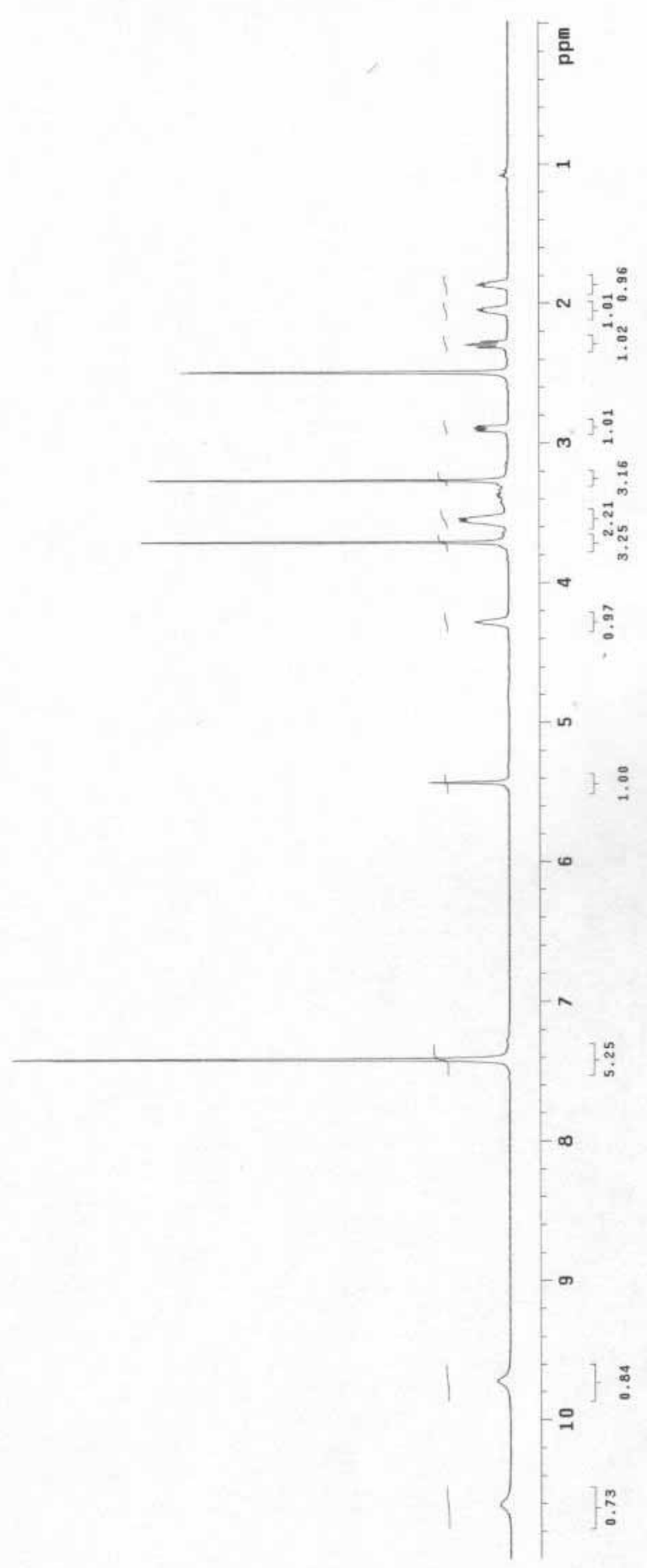




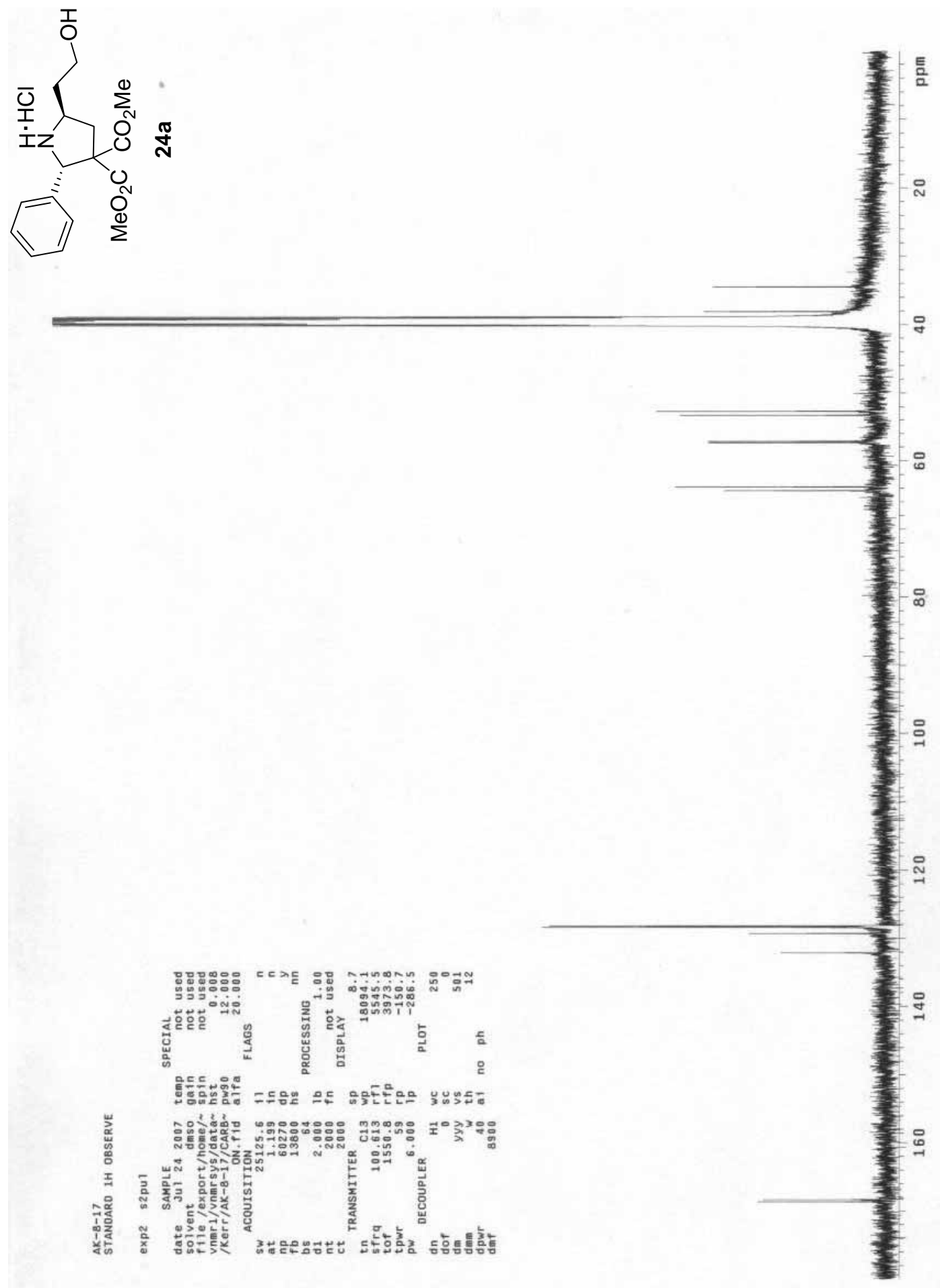




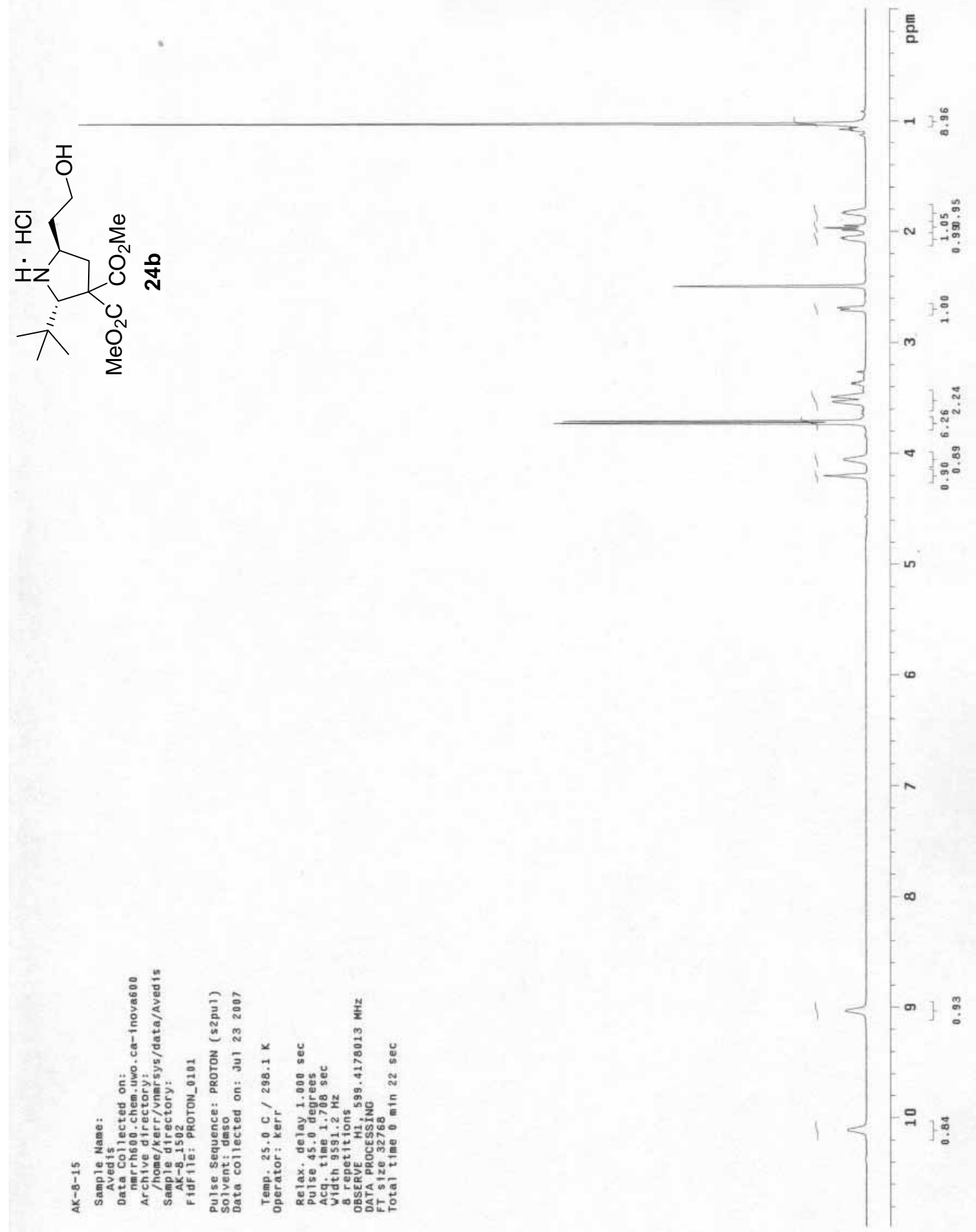




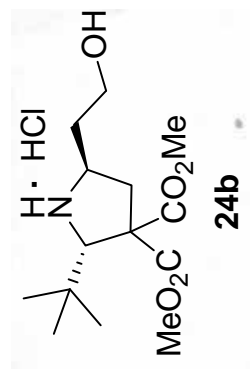

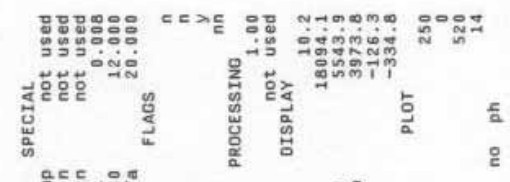

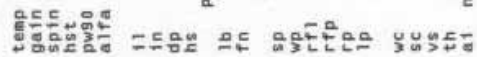

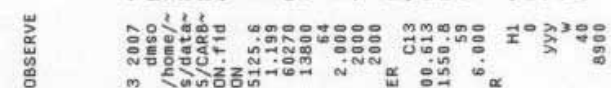

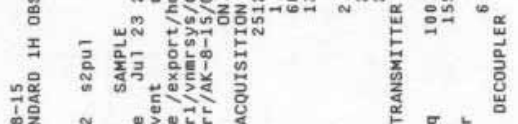

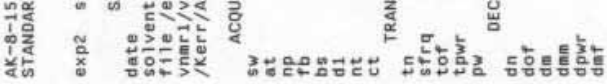




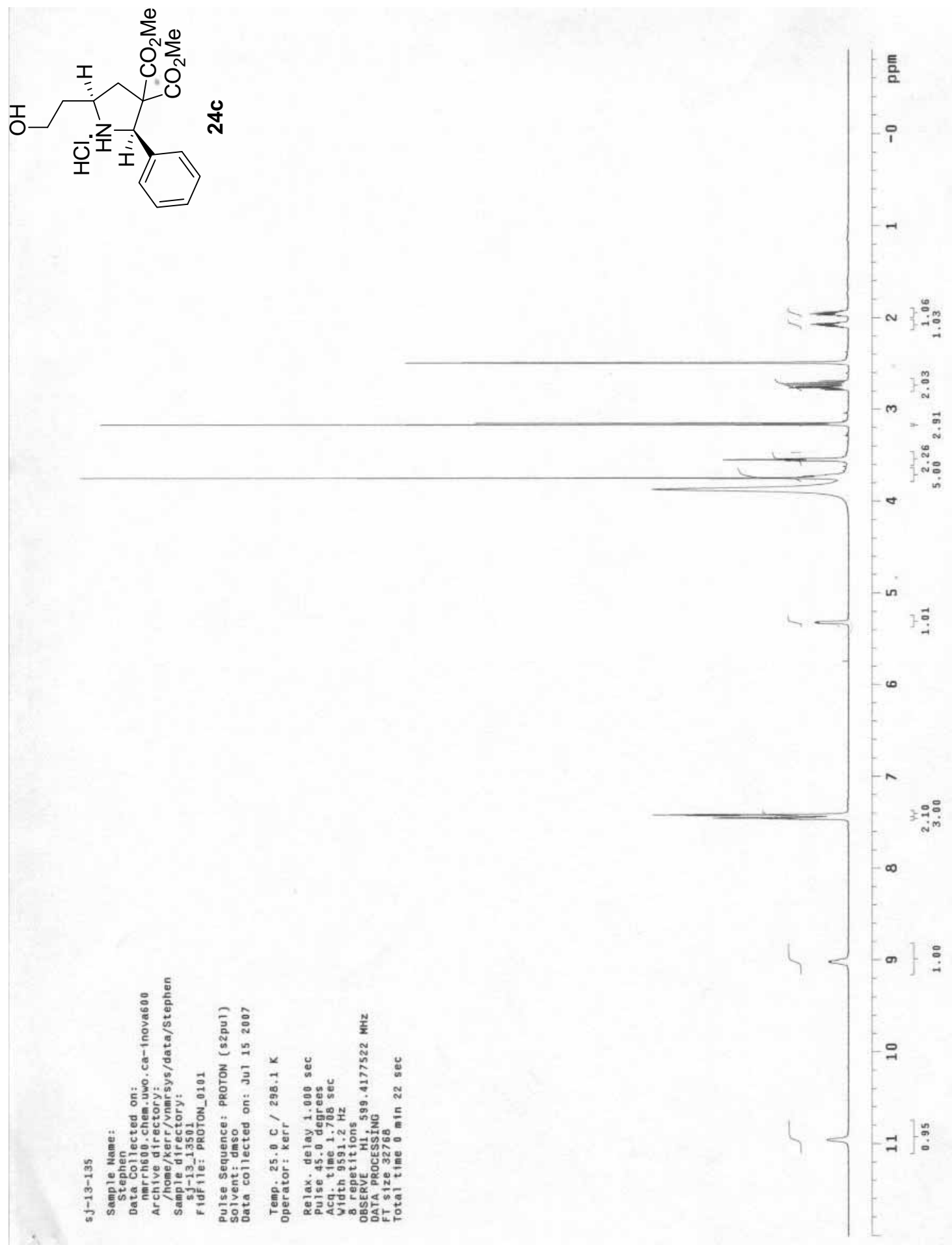



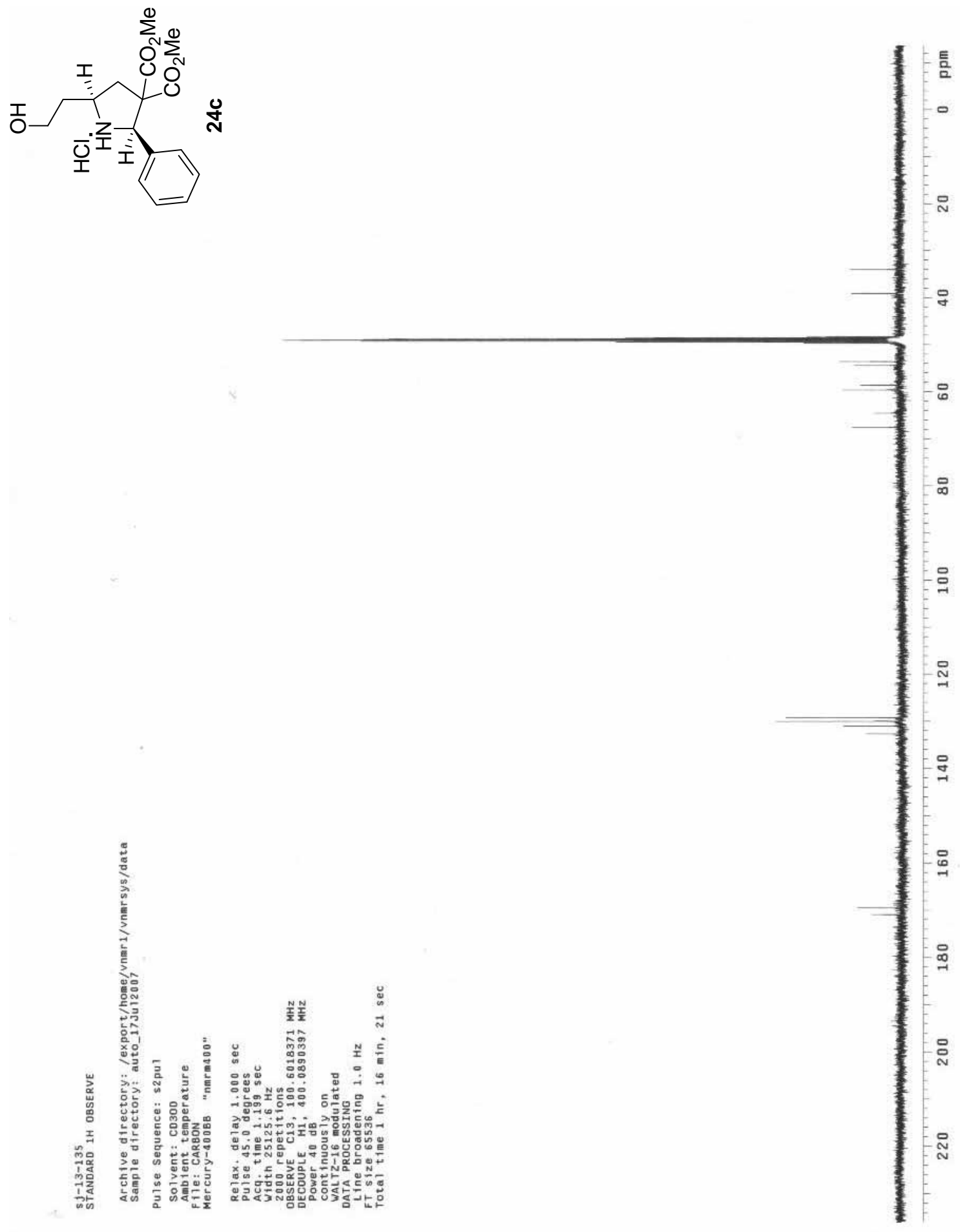

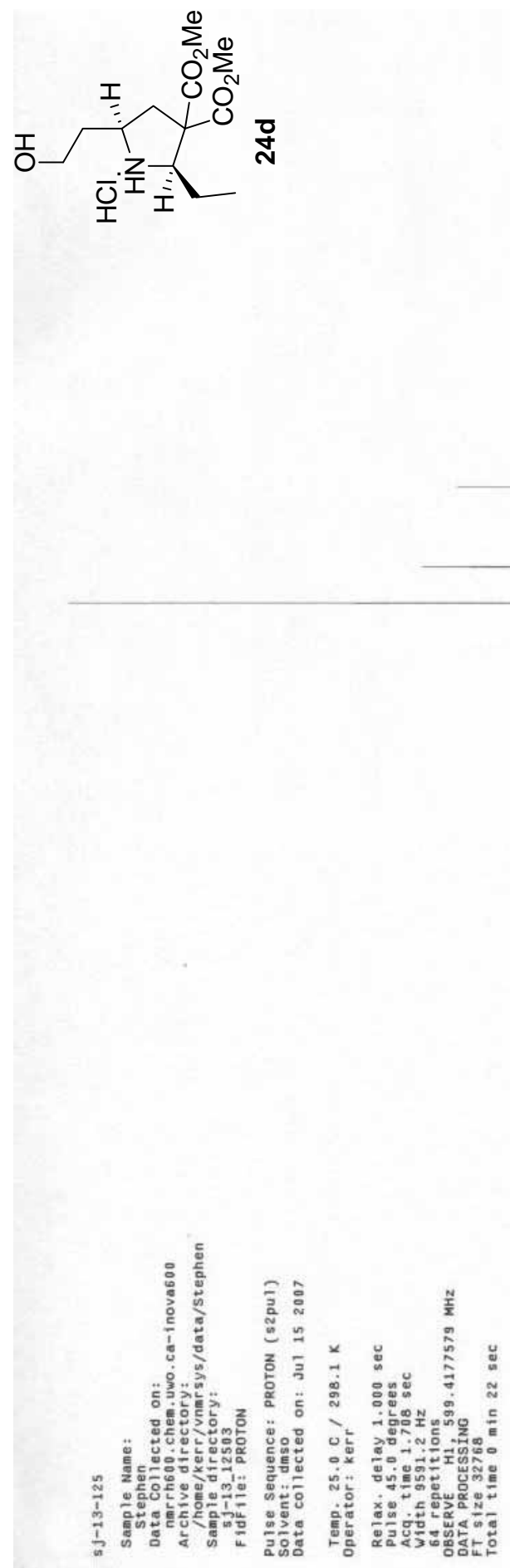

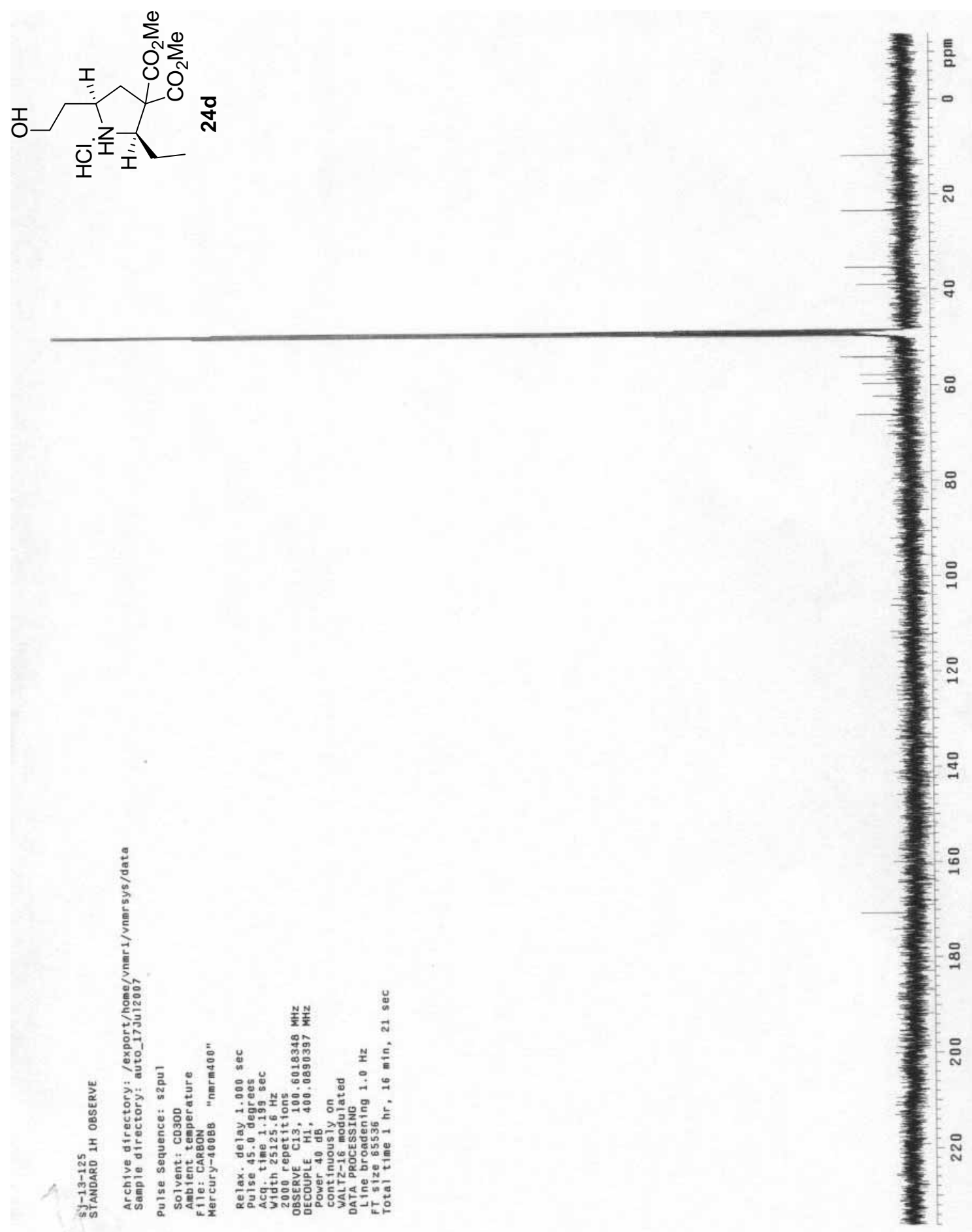

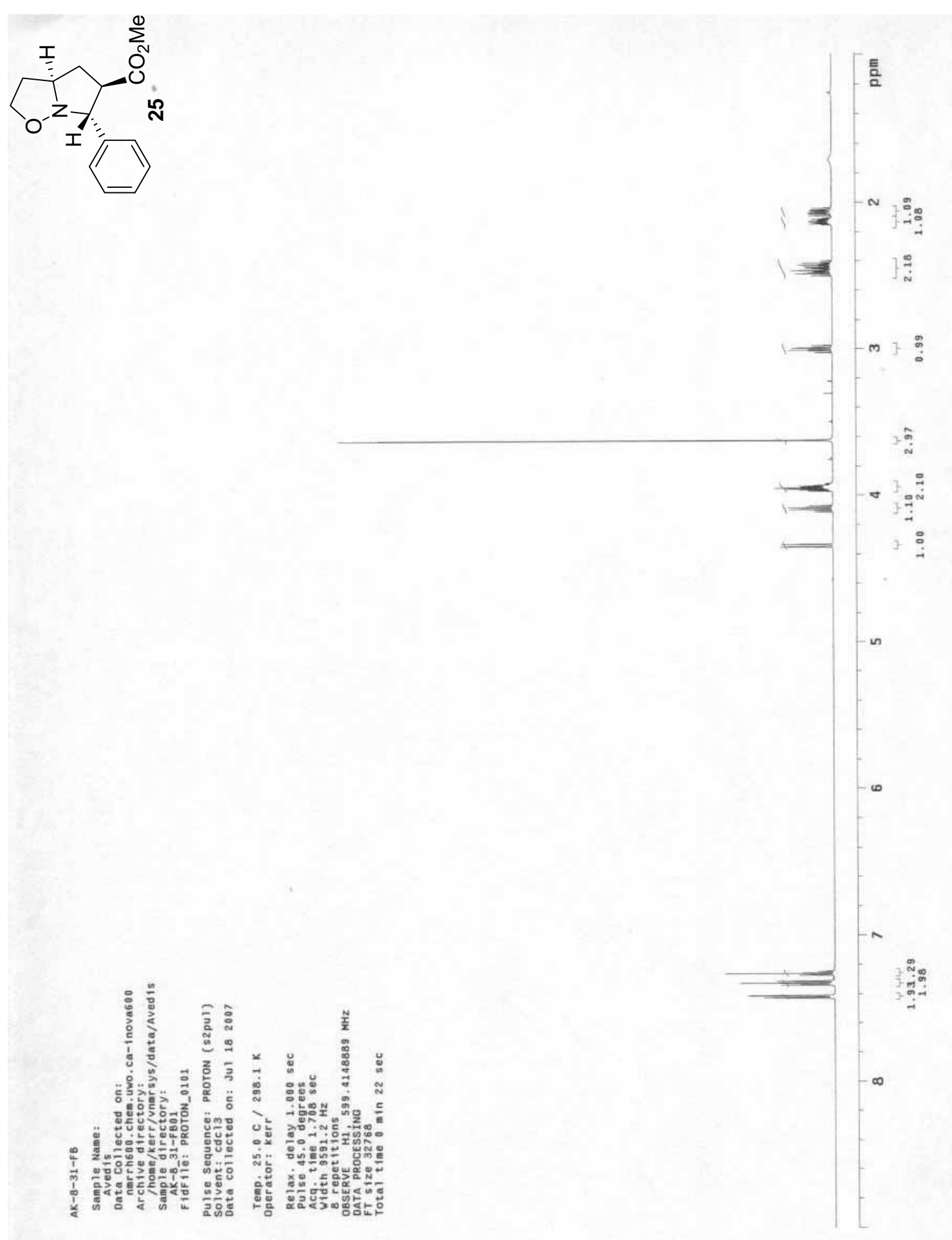

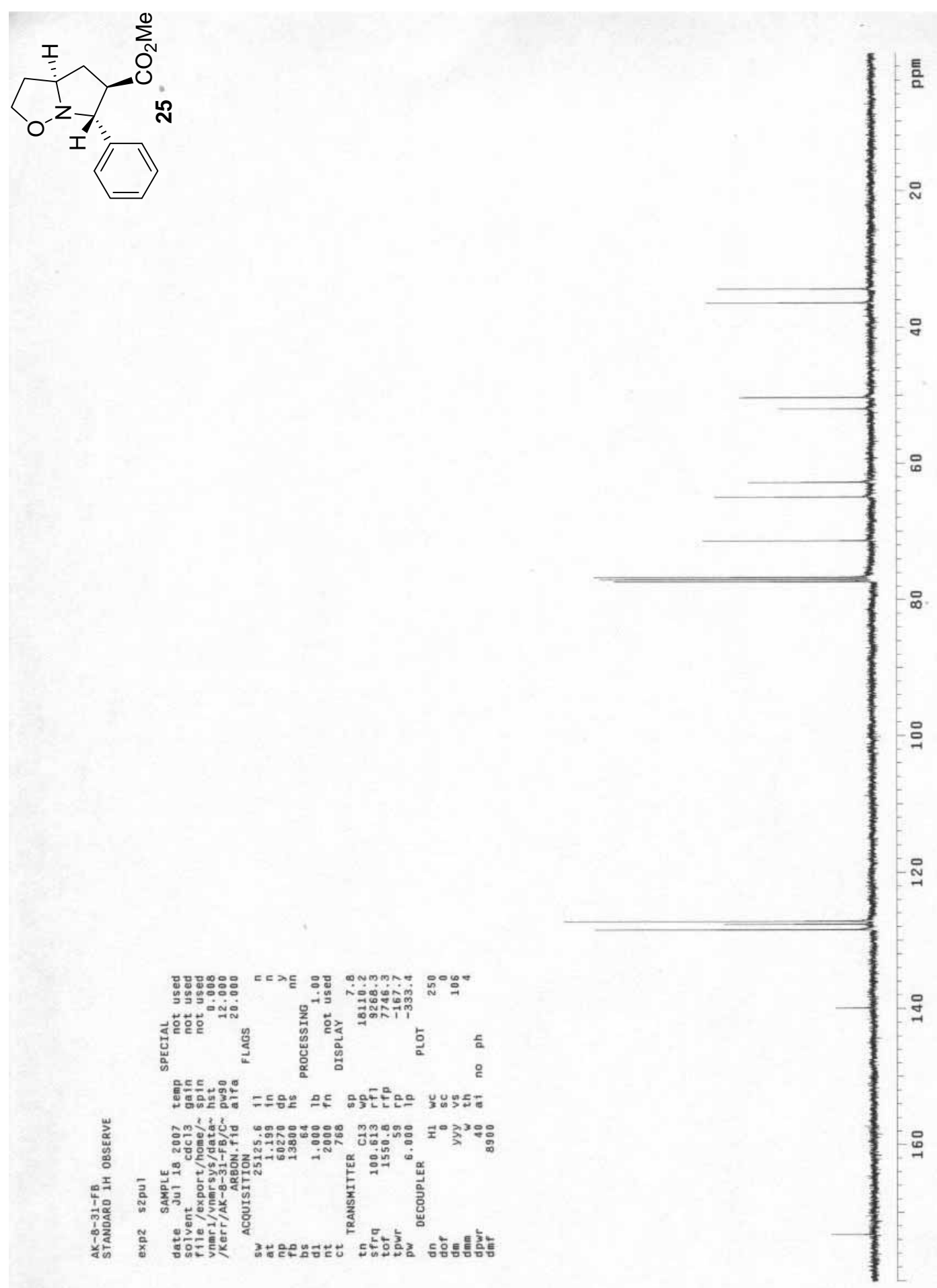


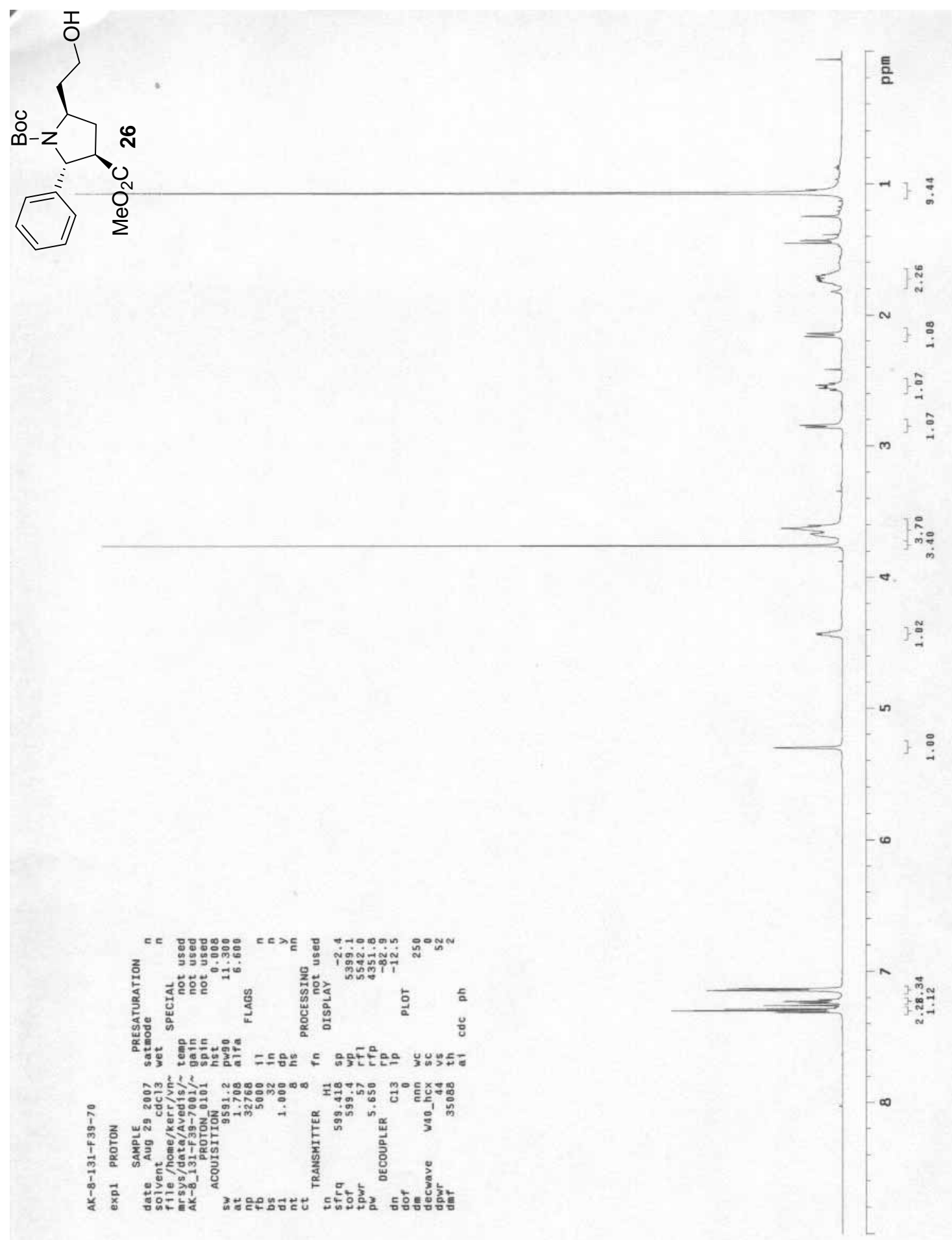



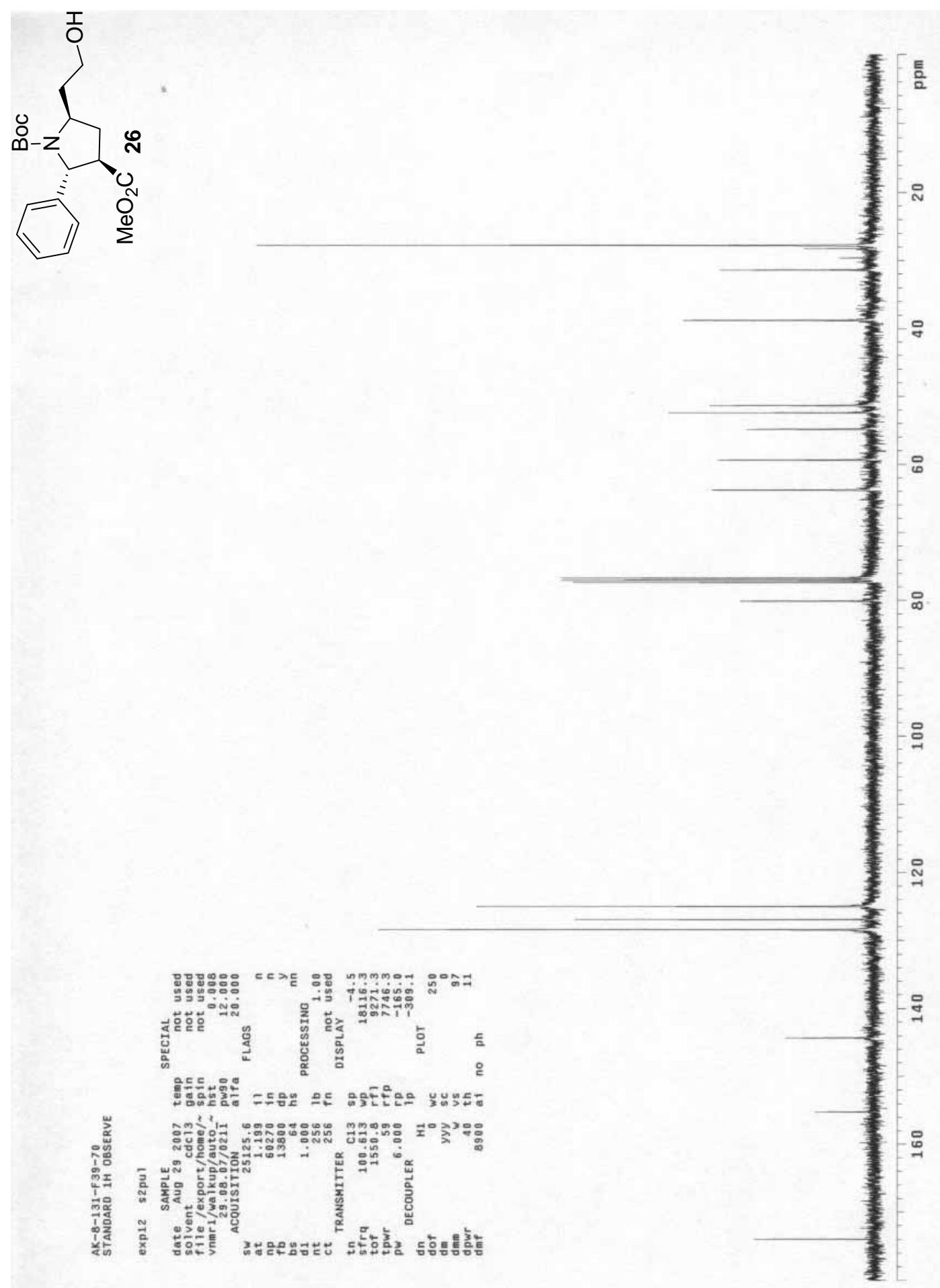


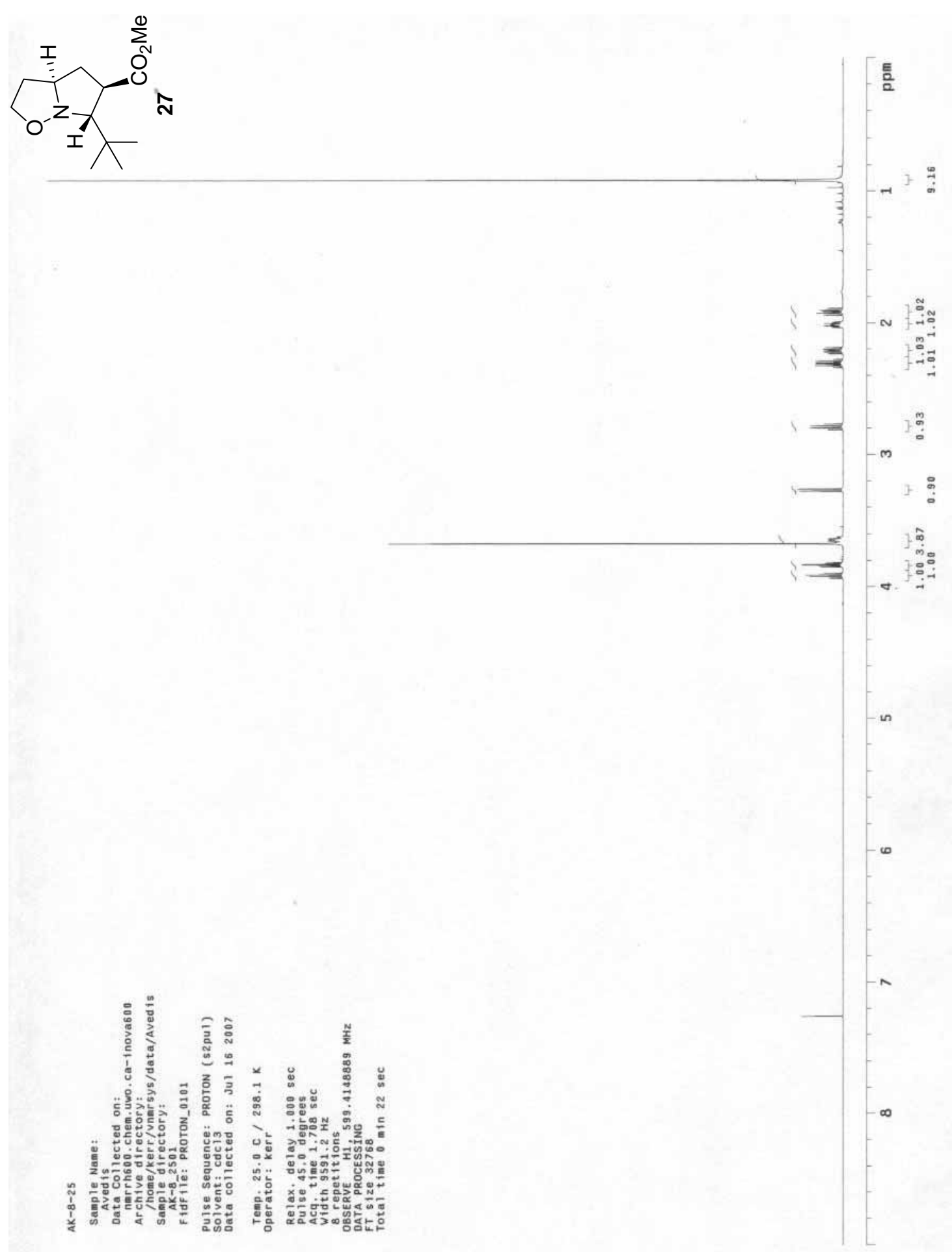



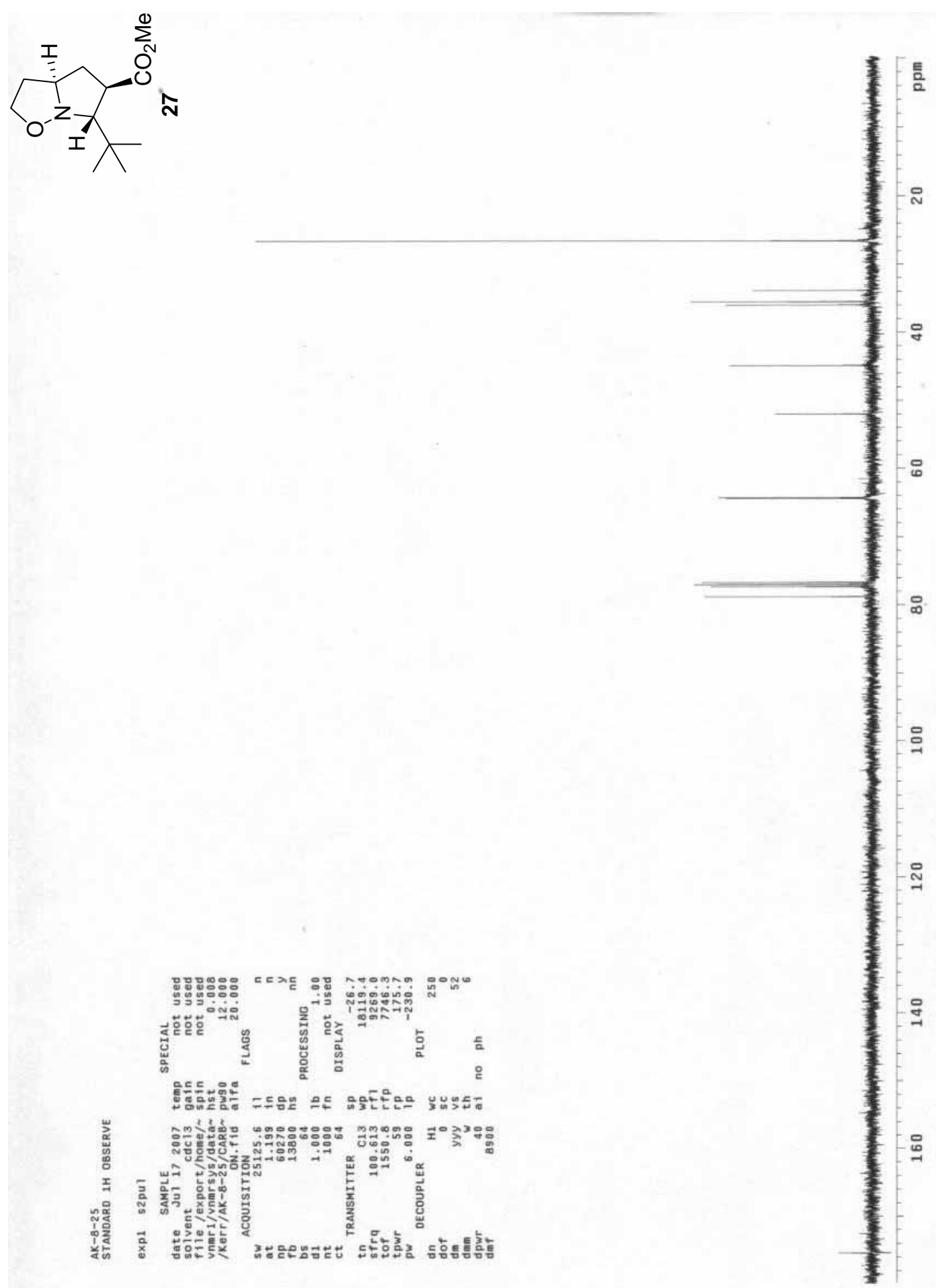


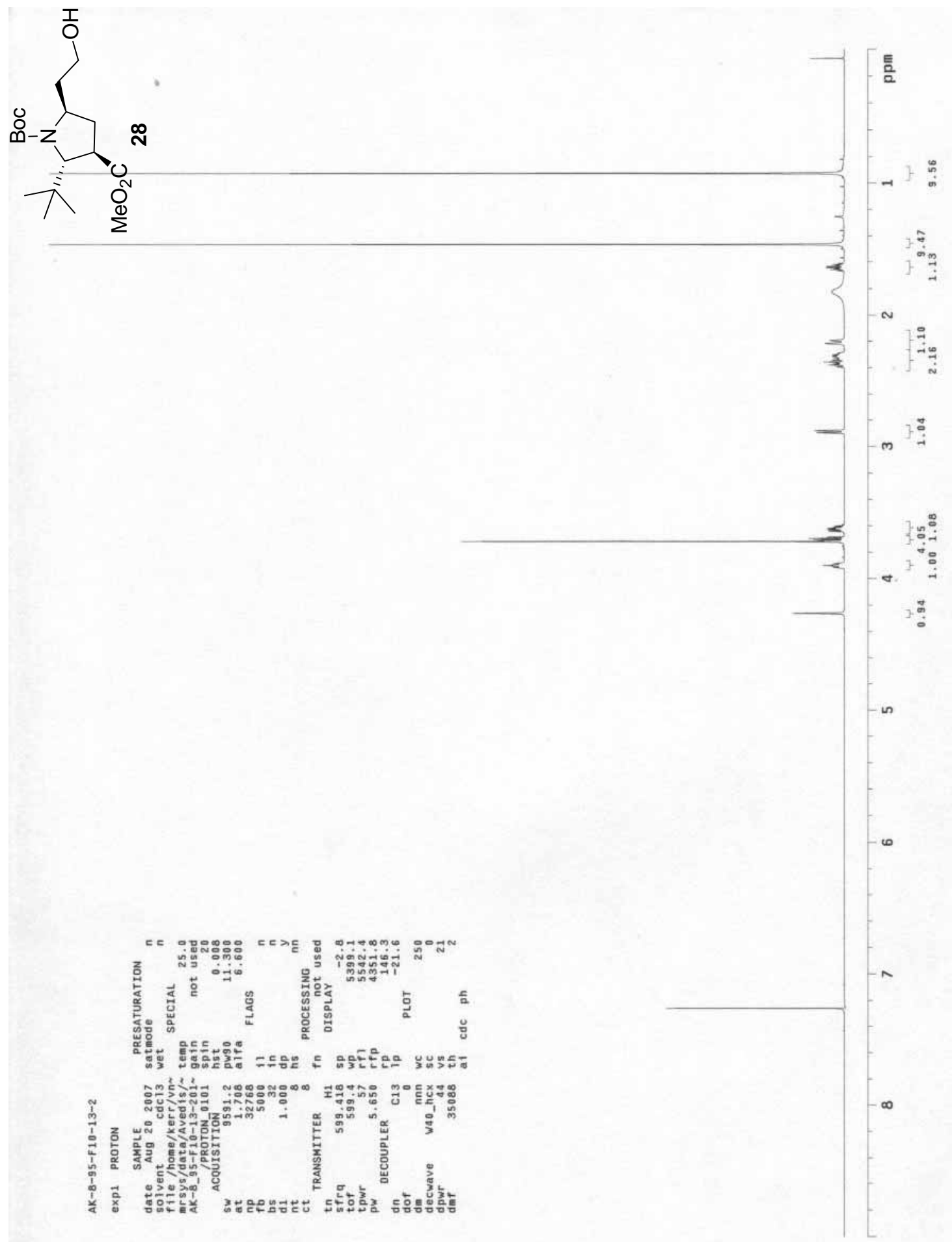



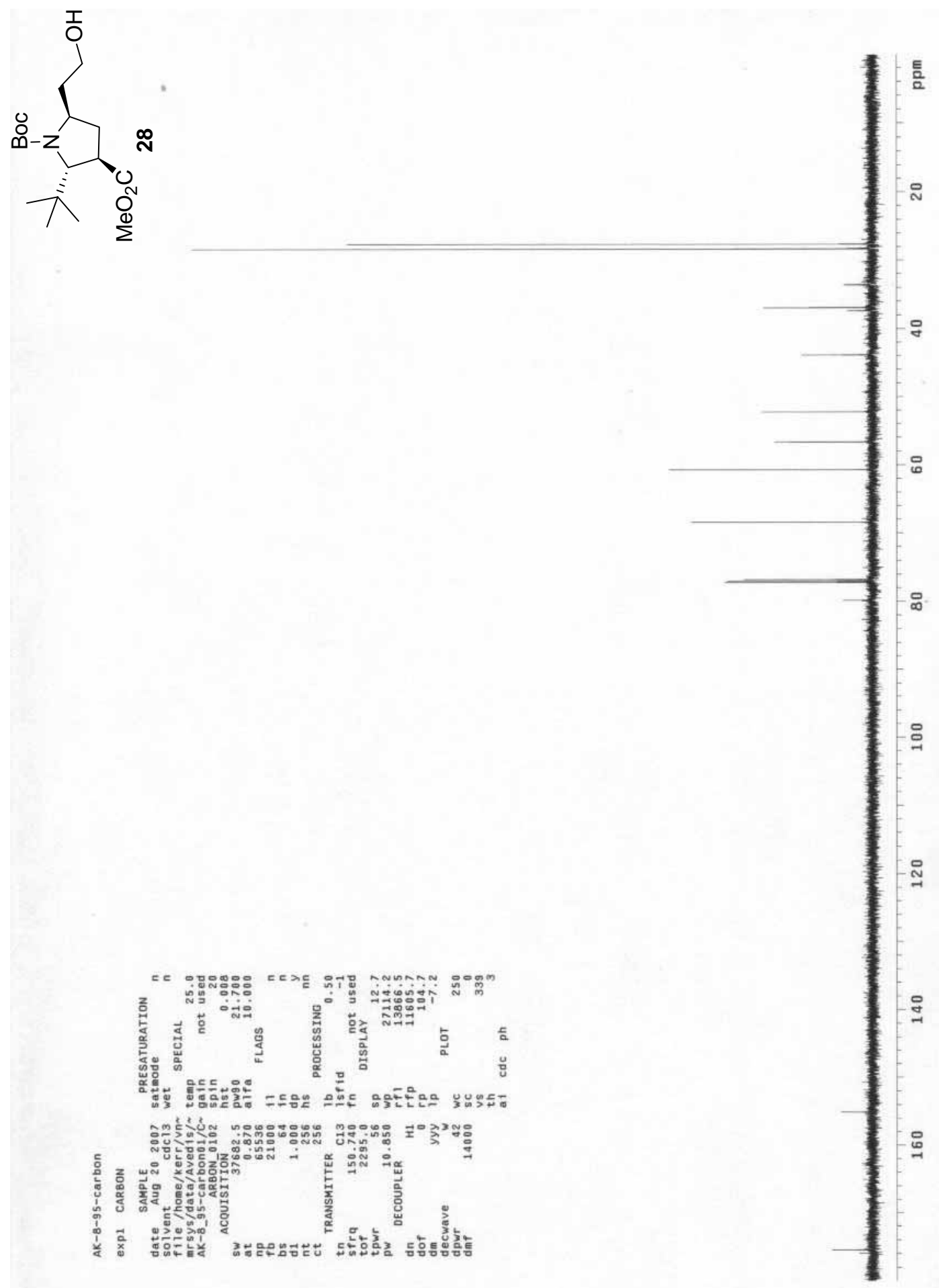

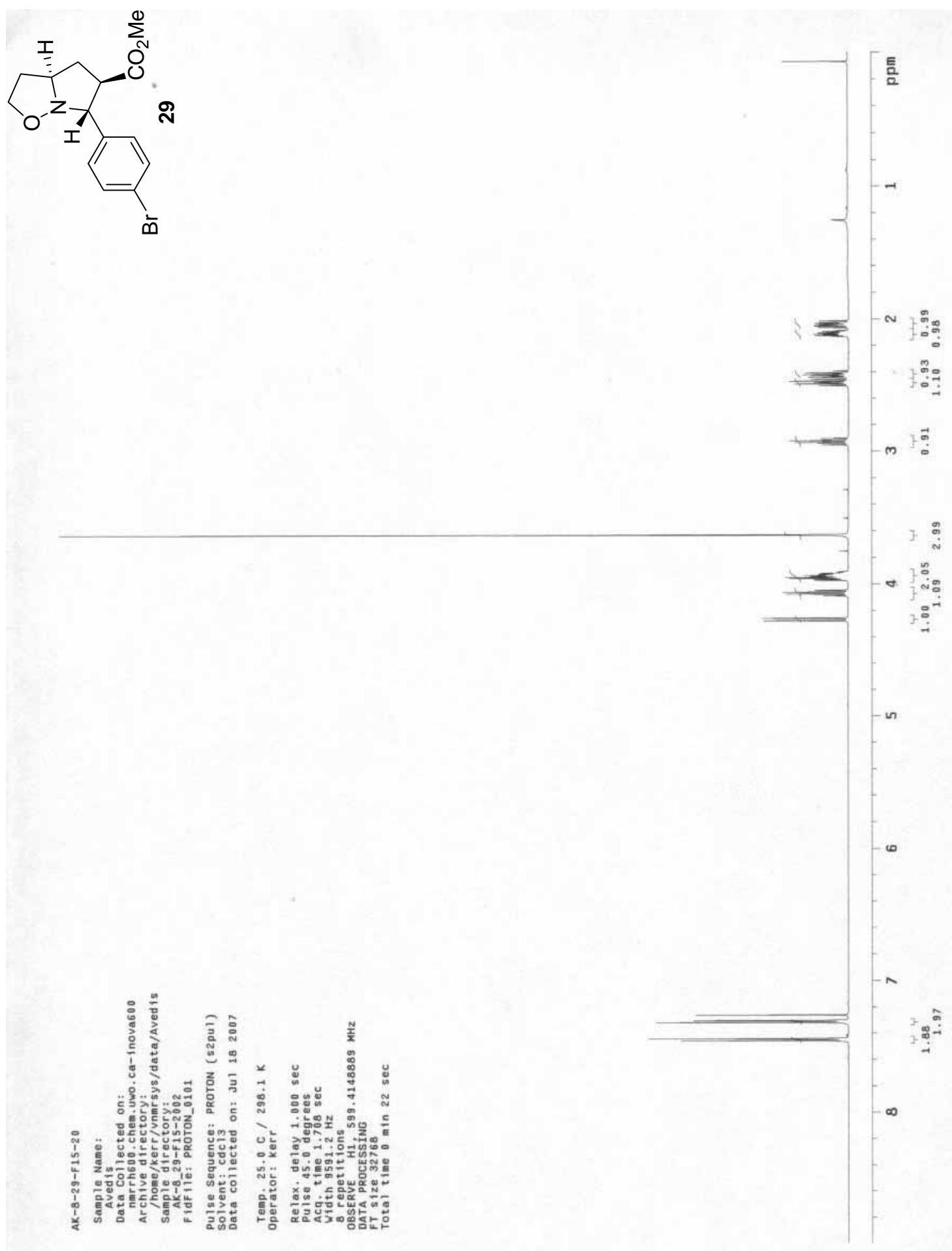

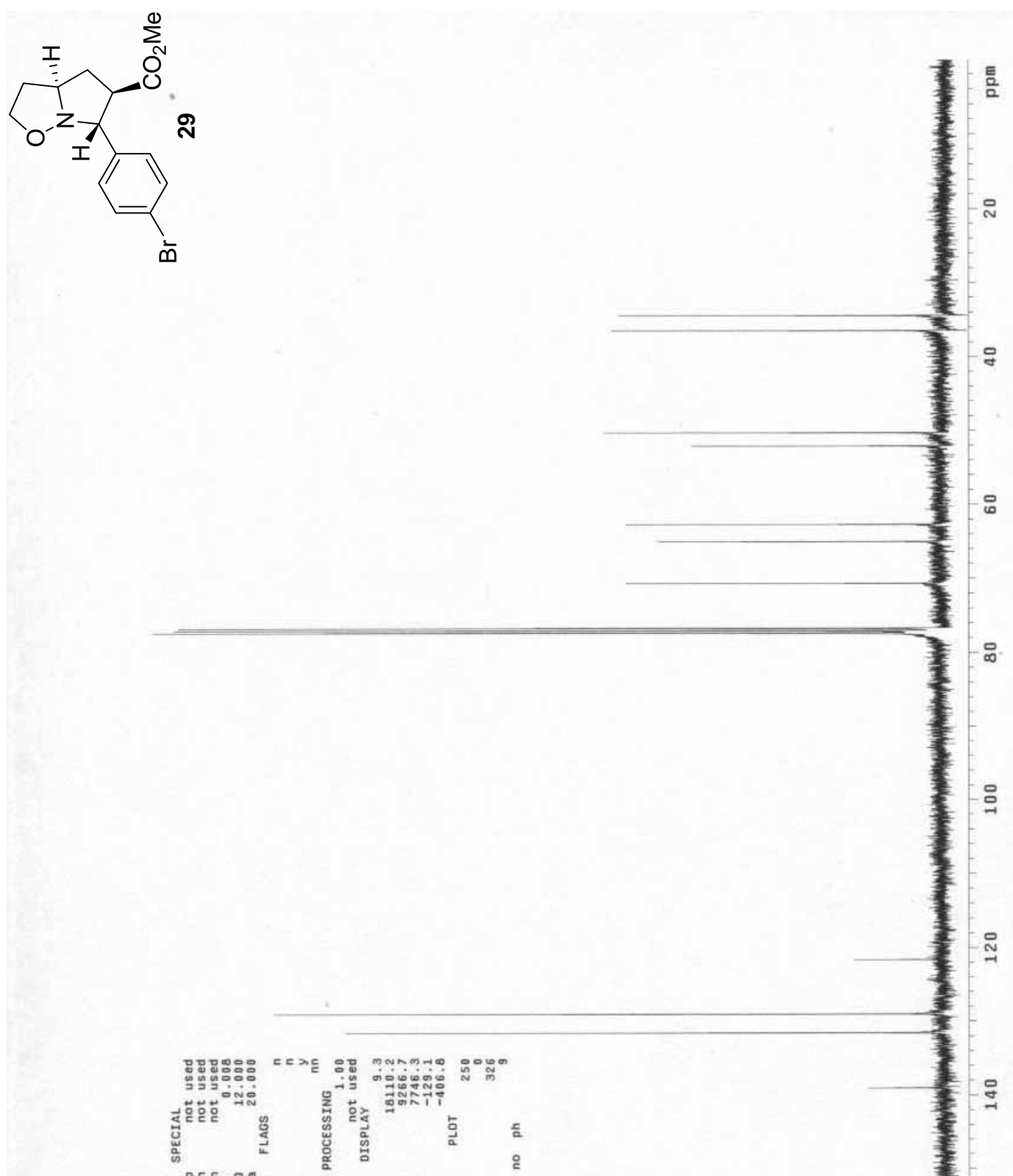

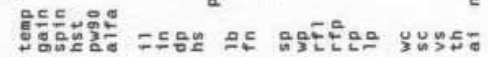

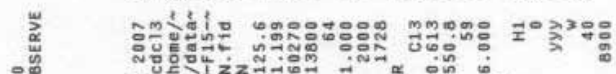

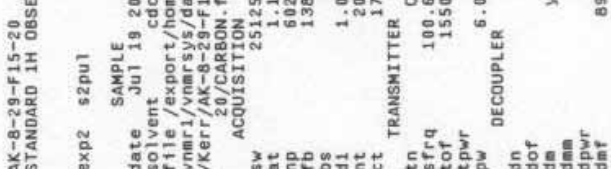



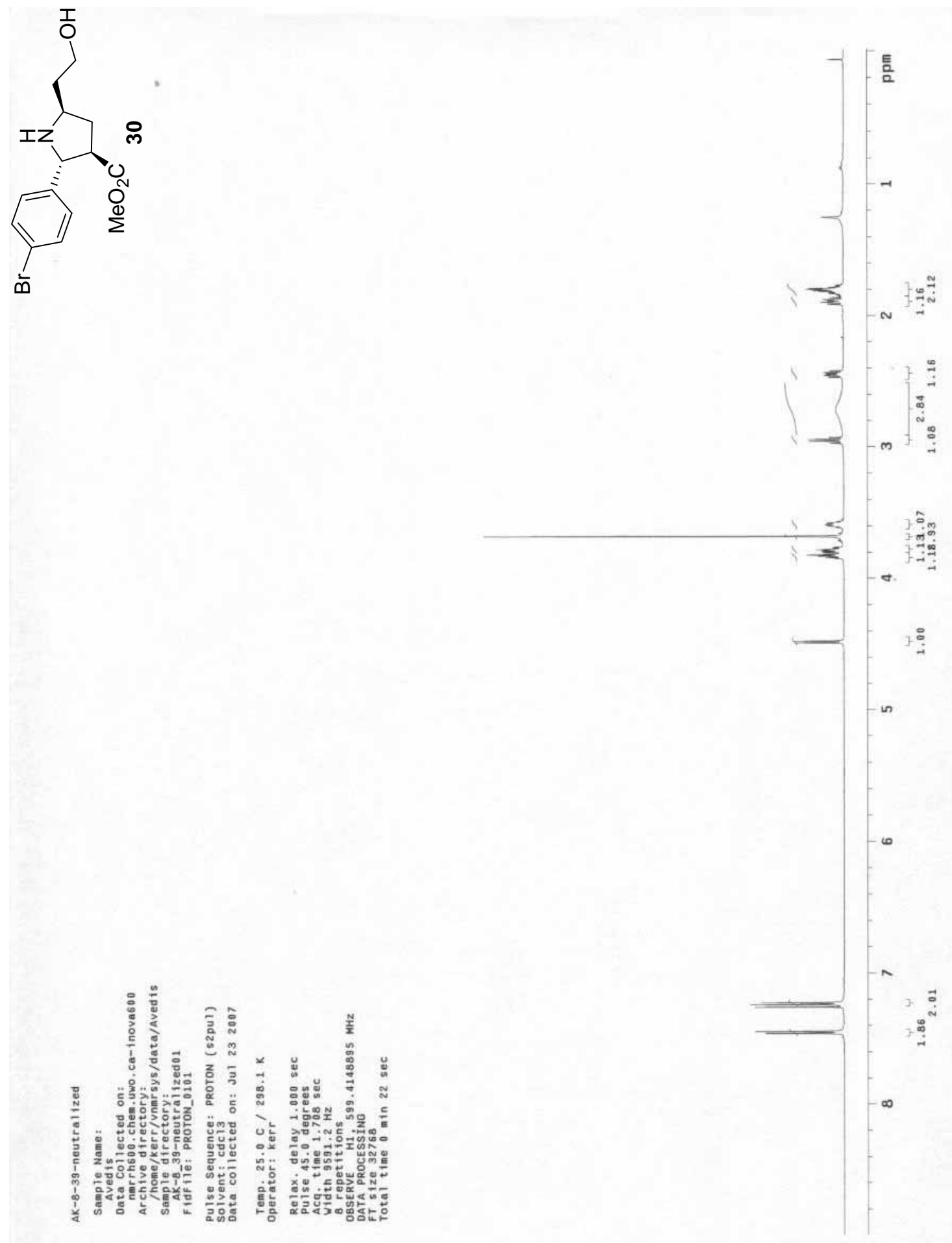

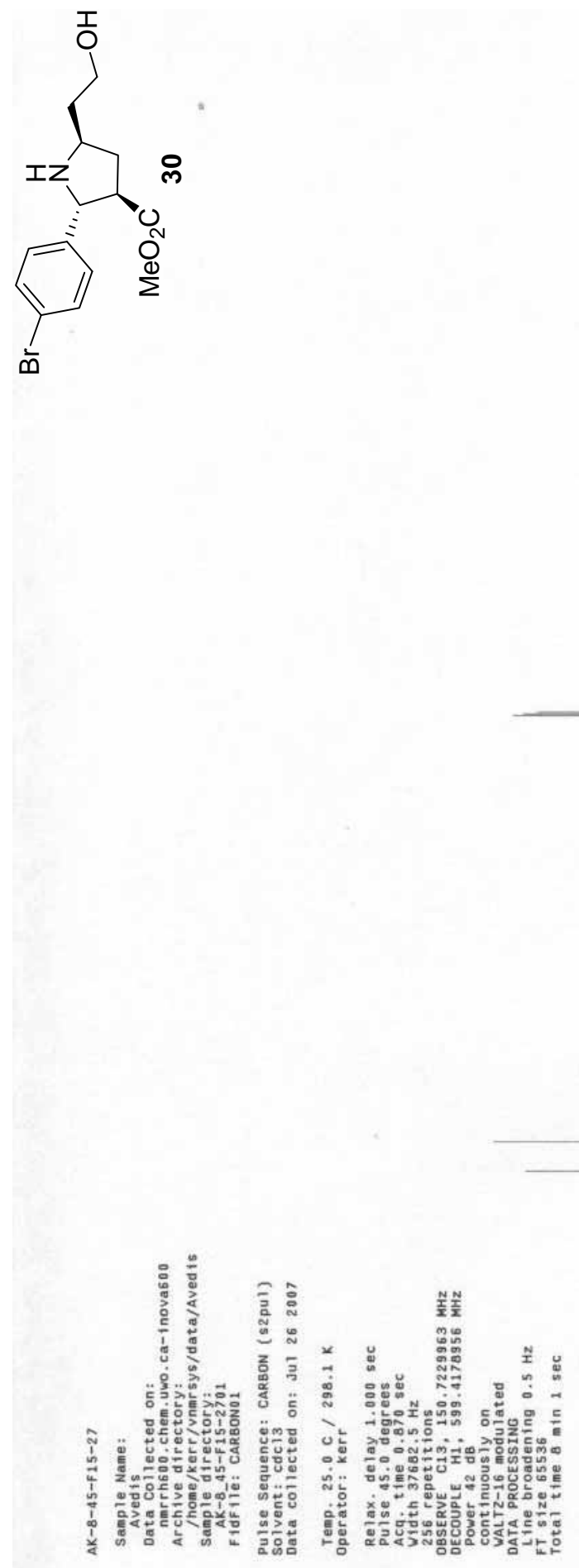